\begin{tabular}{|c|c|}
\hline $\begin{array}{ll}\text { SEP } & 31996_{20} \text { ENGINEERING DATA TRANSMITTAL } \\
\text { Ste. } & 37\end{array}$ & 1. EDT $6176 \frac{1}{28}$ \\
\hline
\end{tabular}

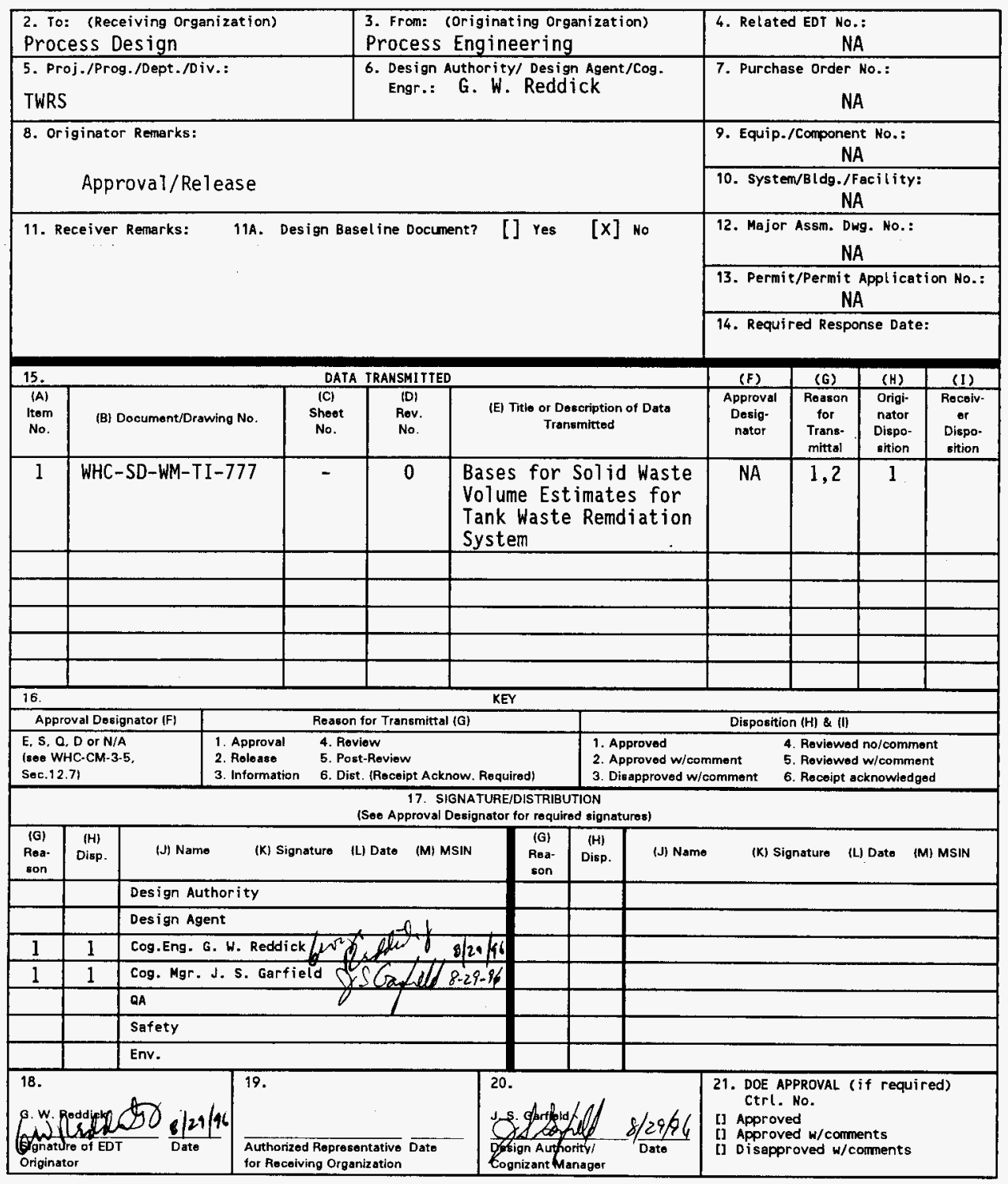

BD-7400-172-2(05/96) GEF097 


\title{
Bases for Solid Waste Volume Estimates for Tank Waste Remediation System
}

\author{
G. W. Reddick
}

Westinghouse Hanford Company, Richland, WA 99352

U.S. Department of Energy Contract DE-AC06-87RL10930
EDT/ECN : 617628
Org Code: 74A20
UC: 721
B\&R Code: EW3130010
Charge Code: D2072
Total Pages: 147

Key Words: TWRS, solid waste, volume estimates, forecast, long-length equipment, TRU

Abstract: This document presents the background and basis for the Tank Waste Remediation System forecast for sol id waste submitted in June 1996. The forecast was generated for single-shell tank and doubleshell tank activities including operations through retrieval and disposal of chemical tank waste.

\footnotetext{
TRADEMARK DISCLAIMER. Reference herein to any specific comercial product, process, or service by trade name, trademark, manufacturer, or otherwise, does not necessarily constitute or imply its endorsement, recomendation, or favoring by the United states Government or any agency thereof or its contractors or subcontractors.

Printed in the United States of America. To obtain copies of this document, contact: WHC/BCS Document Control Services, P.O. Box 1970, Mailstop H6-0B, Richland HA 99352, Phone (509) 372-2420; Fax (509) 376-4989.
}
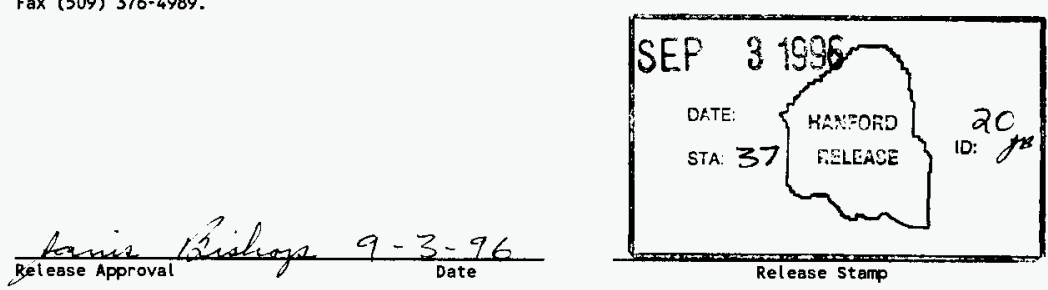
WHC-SD-WM-TI-777

Revision 0

\title{
BASES FOR SOLID WASTE VOLUME ESTIMATES FOR TANK WASTE REMEDIATION SYSTEM
}

\author{
August 1996 \\ G. W. Reddick \\ Westinghouse Hanford Company \\ Prepared for \\ U.S. Department of Energy
}


WHC-SD-WM-TI-777

Revision 0

This page intentionally left blank. 


\section{WHC-SD-WM-TI-777}

Revision 0

CONTENTS

1.0 INTRODUCTION $\ldots \ldots \ldots \ldots \ldots \ldots \ldots \ldots \ldots \ldots \ldots \ldots$

2.0 TANK WASTE REMEDIATION SYSTEM PROGRAM BASES $\ldots \ldots \ldots \ldots \ldots 1$

2.1 SINGLE-SHELL TANK LONG-LENGTH EQUIPMENT $\ldots \ldots \ldots \ldots \ldots 1$

2.2 TANK WASTE REMEDIATION SYSTEM PROCESSING

(PRETREATMENT AND VITRIFICATION) $\ldots \ldots \ldots \ldots \ldots \ldots$

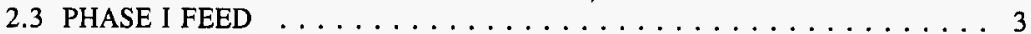

2.4 SINGLE-SHELL TANK RETRIEVAL SEQUENCE $\ldots \ldots \ldots \ldots \ldots \ldots 4$

3.0 SOLID WASTE VOLUME AND CATEGORY BASES $\ldots \ldots \ldots \ldots \ldots \ldots \ldots$

3.1 SINGLE-SHELL TANK LONG-LENGTH EQUIPMENT $\ldots \ldots \ldots \ldots \ldots 7$

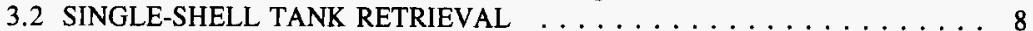

3.3 SOLID WASTE FROM FUTURE FACILITIES $\ldots \ldots \ldots \ldots \ldots \ldots$

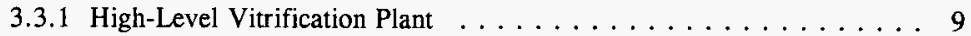

3.3.2 Low-Level Vitrification Plant . . . . . . . . . . . . . 9

3.4 WASTE CATEGORIES . . . . . . . . . . . . . . . . . 9

3.5 DETERMINATION OF TRANSURANIC POTENTIAL FOR TANK

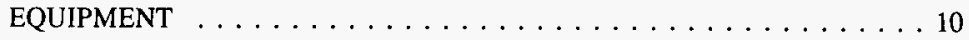

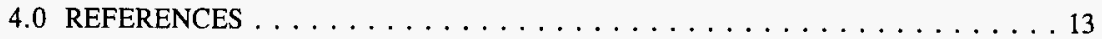

APPENDIX

A - SOLID WASTE FORECAST $\ldots \ldots \ldots \ldots \ldots \ldots \ldots \ldots$ A-1

B - SINGLE-SHELL TANK EQUIPMENT LIST $\ldots \ldots \ldots \ldots \ldots \ldots$ B-1 
WHC-SD-WM-TI-777

Revision 0

\section{LIST OF TERMS}

$\begin{array}{ll}\text { CC } & \text { Complex concentrate } \\ \text { DN } & \text { Dilute non-complexed } \\ \text { DOE } & \text { U.S. Department of Energy } \\ \text { DSS } & \text { Double-shell slurry } \\ \text { DSSF } & \text { Double-shell slurry feed } \\ \text { DST } & \text { Double-shell tank } \\ \text { HLVP } & \text { High-level vitrification plant } \\ \text { HLW } & \text { High-level waste } \\ \text { HWVP } & \text { Hanford Waste Vitrification Plant } \\ \text { LAW } & \text { Low-activity waste } \\ \text { LEC } & \text { Lon equipment container } \\ \text { LLCE } & \text { Long-length contaminated equipment } \\ \text { LLVP } & \text { Low-level vitrification plant } \\ \text { LLW } & \text { Low-level waste } \\ \text { NCAW } & \text { Neutralized current acid waste } \\ \text { NCRW } & \text { Neutralized cladding removal waste } \\ \text { PUREX } & \text { Plutonium-uranium extraction } \\ \text { SST } & \text { Single-shell tank } \\ \text { TRU } & \text { Transuranic } \\ \text { TWRS } & \text { Tank Waste Remediation System } \\ \text { WHC } & \text { Westinghouse Hanford Company } \\ & \end{array}$


WHC-SD-WM-TI-777

Revision 0

\section{BASES FOR SOLID WASTE VOLUME ESTIMATES \\ FOR TANK WASTE REMEDIATION SYSTEM}

\subsection{INTRODUCTION}

This document presents the background and basis for the Tank Waste Remediation System (TWRS) forecast for solid waste submitted in June 1996. The forecast was generated for single-shell tank (SST) and double-shell tank (DST) activities including operations through retrieval and disposal of chemical tank waste. The solid waste forecast prepared using these bases is contained in Appendix A.

\subsection{TANK WASTE REMEDIATION SYSTEM PROGRAM BASES}

The long-length contaminated equipment (LLCE) and other SST retrieval waste are handled according to the agreement reached with the U.S. Department of Energy (DOE 1996).

SST retrieval waste represents the waste generated by operations and maintenance during retrieval operations. Disposal of the equipment used for retrieval is included in the LLCE.

Solid waste will also be generated by the processes that pretreat the waste and the processes that vitrify the waste. These processes are used during the Phase I and Phase II operations described in Section 2.2.

\subsection{SINGLE-SHELL TANK LONG-LENGTH EQUIPMENT}

LLCE will consist of existing equipment and instrumentation used in tanks and equipment for the retrieval of tank waste.

For retrieval of tank waste, each tank will have from one to three sluicers installed. On average each tank will be assumed to have two sluicers installed until more detailed

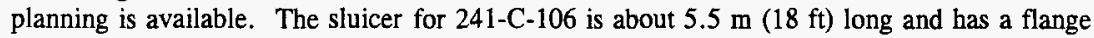
about $79 \mathrm{~cm}$ (31 in.) in diameter.

For retrieval, each tank will have one pump installed for removal of the slurry generated by the sluicers. Final cleanout will probably require the installation of a second pump for heel removal. Some pump failures and replacement are also expected. Two 
pumps are assumed for each tank during sluicing. The pump installed in 241-C-106. is about $9 \mathrm{~m}$ (30 ft) long, collapsible to about $4.5 \mathrm{~m}$ (15 ft). The flange diameter is unknown but should be in the range of 0.76 to $1.14 \mathrm{~m}$ (30 to $45 \mathrm{in}$.).

For 2.84-ML (750,000-gal) and 3.8-ML (1-Mgal) tanks, the sluicers are expected to be the same as the sluicer for 241-C-106. The pumps in the larger tanks will need to be longer. The pumps for $2.84 \mathrm{ML}$ tanks should be collapsible to $7.6 \mathrm{~m}(25 \mathrm{ft}$ ) in length. The pumps

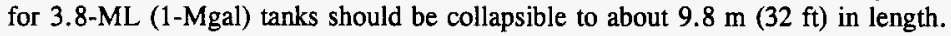

\subsection{TANK WASTE REMEDIATION SYSTEM PROCESSING (PRETREATMENT AND VITRIFICATION)}

Phase I processing represents the demonstration phase and includes retrieval (from DST), in-tank sludge washing, supernatant pretreatment, low-level waste (LLW) immobilization, and an optional high-level waste (HLW) immobilization. Also included are interim storage of immobilized waste and disposal of immobilized waste.

Phase II processing is full scale operation and includes waste retrieval, interim storage of wastes, supernatant pretreatment, sludge/solids pretreatment, LLW immobilization, HLW immobilization, and interim storage of immobilized waste.

Phase I and Phase II use privatized contractor facilities for some of the functions and on site DOE government owned contractor operated facilities for some of the other functions. The amount and type of generated solid waste is expected to be approximately the same regardless of where the privatized line is drawn. In addition, the request for proposal for this work has the DOE responsible for handling the solid waste generated by the privatized facilities.

Phase I privatization begins June 2002 and runs through June 2007; additional operations may occur through 2011 (Washenfelder 1996).

Phase II operations for LLW are 2011 to 2021.

Phase II operations for HLW are 2013 to 2028.

Solid waste from Phase I are returned to DOE. The solid waste generated during Phase I operations are included in the solid waste estimates.

Solid waste generated from Phase II are included in the solid waste estimates.

The solid waste forecast does not include the immobilized tank waste.

The preliminary schedule for transfer of HLW solids to processing and for transfer of low-activity waste (LAW) solutions to the private contractors are used to estimate the kind of 
solid waste generated. Phase I generates some solid waste from equipment failures due the demonstration nature of the processing. Two private contractors may be operating facilities during Phase I. However, the total volume of solid waste generated during Phase I is limited by its relatively short duration.

The preliminary retrieval schedule for SST is used to predict when solid waste is generated by Phase II. The throughput capacity of Phase II facilities is much greater than Phase I. A review of the waste generated by the Plutonium-Uranium Extraction (PUREX) plant in the 1980's is used to assist in the estimation of solid waste from this larger facility.

\subsection{PHASE I FEED}

The sources and volume of Phase I feed are shown below.

Washed high-level waste (HLW) solids:

$\begin{array}{lll}\text { FY 2002 } & 101-\mathrm{AZ} & 380,000 \mathrm{~L}(100,000 \mathrm{gal}) \\ \text { FY 2003 } & 101-\mathrm{AZ}, 102-\mathrm{AZ} & 760,000(200,000) \\ \text { FY 2004 } & 102-\mathrm{AZ} & 760,000(200,000) \\ \text { FY 2005 } & 102-\mathrm{AZ}, 102-\mathrm{AY} & 1,140,000(300,000) \\ \text { FY 2006 } & 102-\mathrm{AY} & 1,140,000(300,000) \\ \text { FY 2007 } & 102-\mathrm{AY} & 1,140,000(300,000) \\ \text { FY 2008 } & 102-\mathrm{AY} & 760,000(200,000)\end{array}$

LAW solution:

FY 2002 DN, DSSF

FY 2003 DSSF, NCRW

FY 2004 DSSF

FY 2005 DSSF

FY 2006 DSSF

FY 2007 NCAW, CC

FY 2008 CC

FY 2009 DSS

FY 2010 CC

FY 2011 NCAW

\section{$7.7 \mathrm{ML}$}

8.4

6.3

2.9

3.8

5.0

3.9

3.5

4.4

1.5

$\mathrm{CC}=$ Complexant concentrate

DN $=$ Dilute, non-complexed

DSS $=$ Double-shell slurry

DSSF $=$ Double-shell slurry feed

NCRW and NCAW = the supernatants from the respective NCAW and NCRW tanks. 
WHC-SD-WM-TI-777

Revision 0

\subsection{SINGLE-SHELL TANK RETRIEVAL SEQUENCE}

Retrieval of waste from SSTs begins in fiscal year 2004 and continues through 2018. Below is the initiation year for SST retrieval by tank farm.

FY 2004 AX 1 tank

A 1 tank

C 2 tanks

FY 2005 A 2 tanks

SX 1 tank

AX 2 tanks

FY 2006 SX 1 tank

FY 2007 SX 3 tanks

FY 2008 SX 2 tanks

U 6 tanks

FY 2009 C 4 tanks

U 2 tanks

AX 1 tank

FY 2010 C 7 tanks

A 1 tank

U 1 tank

FY 2012 A 2 tanks

B 4 tanks

BX 4 tanks

BY 2 tanks

C 2 tanks

S 2 tanks

SX 2 tanks

T 5 tanks

TX 2 tanks

TY 2 tanks

FY 2013 BY 2 tanks

SX 2 tanks

T 4 tanks

TX 4 tanks

TY 3 tanks 


\section{Revision 0}

FY 2014 B 1 tanks

BX 2 tanks

BY 3 tanks

S 2 tanks

SX 3 tanks

T 5 tanks

TX 5 tanks

TY 1 tanks

FY 2015 B 1 tank

BX 1 tank

BY 2 tanks

S 3 tanks

TX 1 tank

U 2 tanks

FY 2016 B 4 tanks

BX 3 tanks

BY 2 tanks

S 3 tanks

SX 1 tank

T 2 tanks

TX 6 tanks

FY 2017 B 6 tanks

BX 2 tanks

BY 1 tanks

S 2 tanks

U 5 tanks

(This count of tanks includes both 100 and 200 series tanks. The 200 series tanks are four each in B Farm, C Farm, T Farm, and U Farm. Waste from 241-C-106 was assumed to be previously retrieved.) 
WHC-SD-WM-TI-777

Revision 0

This page intentionally left blank. 
WHC-SD-WM-TI-777

Revision 0

\subsection{SOLID WASTE VOLUME AND CATEGORY BASES}

\subsection{SINGLE-SHELL TANK LONG-LENGTH EQUIPMENT}

The long-length equipment for disposal consists of existing installed equipment plus the sluicers and pumps used for retrieval.

The existing long-length equipment in SST was identified by tank farm, type and number of equipment pieces. The total number of equipment pieces in SST was estimated to be 860 (Galbraith 1996). The table of these equipment pieces is contained in Appendix B. All of these equipment pieces were assumed to be removed before retrieval equipment is installed. This conservative assumption produced a total volume of waste that is less than the number estimated in previous forecasts. This equipment was allocated to individual tanks farms based on the estimated existing equipment inventory.

$\begin{array}{lc}\text { A Farm } & 85 \text { equipment pieces } \\ \text { AX Farm } & 65 \\ \text { B Farm } & 33 \\ \text { BX Farm } & 76 \\ \text { BY Farm } & 101 \\ \text { C Farm } & 96 \\ \text { S Farm } & 52 \\ \text { SX Farm } & 110 \\ \text { T Farm } & 51 \\ \text { TX Farm } & 89 \\ \text { TY Farm } & 2 \\ \text { U Farm } & 100\end{array}$

Some of this equipment may not need to be removed to support retrieval of tank waste. At least three to four risers per tank are expected to be utilized for retrieval equipment and instrumentation. Existing equipment in the risers to be used by retrieval operations will require removal. Some equipment in other risers may interfere with sluicer operations and will also require removal. Under the best of conditions, the total count of existing equipment pieces requiring removal is expected to be 400 to 500 . When specific plans are established for retrieval equipment on a tank-by-tank basis, refined estimates of the solid waste volume can be prepared.

The length and diameter of equipment was estimated based on installed equipment lists for each farm (Roach 1995). Based on the equipment sizes, the number of each container size was identified for each tank farm. A standard "square long equipment container (LEC)" was used for each piece of equipment. The LEC dimensions and volumes were provided in the solid waste spreadsheet (SWIFT) provided by solid waste programs.

The schedule for the removal of the equipment was determined based on the latest 


\section{Revision 0}

estimate of the SST retrieval sequence (Penwell 1996). The retrieval sequence is estimated by tank. For purposes of the forecast, the sequence was identified by tank farm. As an example, in 2004, retrieval of waste from one A Farm tank begins, retrieval from one AX Farm tanks begins, and retrieval from two $\mathrm{C}$ Farm tanks begins. The equipment in these tanks was assumed to be removed in 2001, three (3) years before retrieval is scheduled to begin. Likewise for all of the SST, the existing equipment is removed three (3) years before retrieval is scheduled to begin.

With an estimate of the equipment in each tank farm and how many tanks are to be retrieved per year, the removal of equipment was prorated for each year from 2001 through 2014. (SST retrieval starts in the tanks from 2004 through 2017.)

Other long-length equipment will be generated by the disposal of retrieval equipment, sluicers and pumps. From one to three sluicers and one pump are expected to be used in each tank for retrieval operations. Of these, about one sluicer per tank and one pump per tank was assumed to require disposal as long-length equipment. This set of assumptions is expected to be conservative because some sluicers and pumps will be reused and those that are not reused are expected to be left in the tanks and disposed in the same manner as the tanks (DOE 1996).

All of the retrieval pumps and sluicers have some capability for retraction that permits a shorter container can be used for storage and disposal. All of the retrieval equipment is expected to fit into the containers that are $8.53-\mathrm{m}$ (336-in.) long except the pumps removed from the $3,800 \mathrm{~m}^{3}$ (1 Mgal) tanks which will require containers $12.8 \mathrm{~m}(504 \mathrm{in}$.) long. In total, 140 sluicers and 140 pumps were identified for disposal as solid waste.

The schedule for the retrieval equipment disposal was assumed to be the same as the start date for retrieval. This will conservatively assure that containers are available and storage space is allocated when needed.

\subsection{SINGLE-SHELL TANK RETRIEVAL}

The solid waste in the SST retrieval category is the waste generated by SST retrieval operations. The volume of solid waste used in the this forecast is the same as used in the previous forecast. The volume of waste was prorated over the retrieval schedule based on the number of tanks being retrieved per year.

\subsection{SOLID WASTE FROM FUTURE FACILITIES}

The future facilities for processing and disposal of tank chemical waste are not well defined. In about two years, conceptual designs for the Phase I facilities are scheduled to be completed. As the conceptual designs are completed and progress is made toward more detailed designs, the accuracy of estimates for solid waste generation should improve. Until 
designs are available, estimates are very preliminary and have to estimated based on knowledge of former processing facilities such as PUREX or B Plant. The feed rates required for the future facilities provides some measure of the size of facilities (Slaathaug 1996). In addition, the designs of canceled projects such as the Hanford Waste Vitrification Plant (HWVP) and Interim Pretreatment Module (IPM) provide a general idea of the equipment and processes that are expected in future facilities.

\subsubsection{High-Level Vitrification Plant}

The solid waste from the high-level vitrification plant (HLVP) was estimated based on the annual production of solid waste by the PUREX plant during operations in the 1980's. The Phase II operations include a large facility and is expected to generate an amount of waste equivalent to the PUREX plant. The PUREX plant typically generated about $1,600 \mathrm{~m}^{3}$ of solid waste per year in the 1980's. The HLVP is expected to include some pretreatment processes and solid wastes generated by these processes are included in the forecasted volumes. In previous solid waste forecasts, the pretreatment processes had been included in a separate facility. The Phase I plant is a demonstration facility and is expected to generate about one fifth as much solid waste on an annual basis as the Phase II facility.

\subsubsection{Low-Level Vitrification Plant}

The Phase II low-level vitrification plant (LLVP) is a very large facility and is expected to generate an amount of waste equivalent to twice the volume of solid waste generated by the PUREX plant. The LLVP will have pretreatment processes and the solid waste generated by these processes are included in the forecasted volumes. In previous solid waste forecasts, the pretreatment processes had been included in a separate facility. The Phase I plant is a demonstration facility and is expected to generate about one fifth as much solid waste on an annual basis as the Phase II facility.

\subsection{WASTE CATEGORIES}

In addition to the volume, the solid waste forecast requires the type of waste be identified by hazard category and radionuclide category. All of the solid waste from the tanks were considered to be mixed waste, having both a hazardous component as defined by RCRA and a radionuclide component. Most of the waste coming from tanks is considered to be remote handled except for tanks that largely contain transuranic (TRU) waste without a large concentration of radioactive cesium and strontium.

Retrieval operations, the high level vitrification plant, and the low level vitrification plant have both contact handled and remote handled waste. The breakout of waste into the various categories was based on past solid waste forecasts (Valero 1996) and waste generated by former plants (Duncan 1993). 
Most of the curies in SST are ${ }^{90} \mathrm{Sr}$ and ${ }^{137} \mathrm{Cs}$. These two elements and their daughters make up between 95 percent and 99 percent of the total curies. Most of the equipment removed from tanks will be greater than the category I limit for these radionuclides but less than the category III limit. The category I limit for ${ }^{90} \mathrm{Sr}$ is $4.3 \times 10^{-3} \mathrm{Ci} / \mathrm{m}^{3}$. The category III limit is $1.5 \times 10^{4} \mathrm{Ci} / \mathrm{m}^{3}(15 \mathrm{Ci} / \mathrm{L})$. The category I limit for ${ }^{137} \mathrm{Cs}$ is $6.3 \times 10^{-3} \mathrm{Ci} / \mathrm{m}^{3}$. The category III limit is $1.3 \times 10^{4} \mathrm{Ci} / \mathrm{m}^{3}(13 \mathrm{Ci} / \mathrm{L})$. The sum of the fractions rule is used to determine the category when more than one radionuclide is present.

Waste from the retrieval operations and the vitrification plants will include some category I waste and some category III waste. None of the waste from the tanks, retrieval operations, or the vitrification plants is expected to be greater than category III. The highest concentration of cesium in any one tank is about $2 \mathrm{Ci} / \mathrm{L}$ in DST 241-AZ-101. The highest concentration of strontium is about $7 \mathrm{Ci} / \mathrm{L}$ (in about $867,000 \mathrm{~L}$ ) in SST 241-C-106. Retrieval operations and other processing will generally tend to dilute the radionuclide concentrations. Some exceptions to this rule may occur during pretreatment processes, but the category III limit should not be exceeded for any solid waste generated during the operation of these processes.

\subsection{DETERMINATION OF TRANSURANIC POTENTIAL FOR TANK EQUIPMENT}

The amount of TRU equipment removed from tanks is significantly affected by an agreement reached with the DOE (1996). Equipment that has not been removed from tanks would be included in the closure plans for the tanks. All equipment removed from SSTs after 2003 will be decontaminated to non-TRU levels. To determine the amount of TRU equipment removed from tanks through 2003, the inventory of solid chemical waste in each of the SSTs was reviewed. Most of the curies of TRU constituents are contributed by plutonium and americium. Very few of the SSTs have a combination of TRU solids and a significant sludge depth.

In order for heavy equipment removed from a tank to be classified as TRU, the concentration of TRU must be $100 \mathrm{nCi} / \mathrm{g}\left(1 \times 10^{-7} \mathrm{Ci} / \mathrm{g}\right)$ of total waste. The total weight of the equipment and any contamination is considered in making the TRU designation (DOE 1995). Tank equipment varies in weight from a few hundred pounds (e.g., $150 \mathrm{lbs}$ to 500 $\mathrm{lbs}$ ) to several thousand pounds ( $150 \mathrm{lbs}$ is $68,000 \mathrm{~g} ; 5,000 \mathrm{lbs}$ is 2.26 million g). To contaminate $150 \mathrm{lbs}$ of waste to TRU levels would require only 0.007 curies of TRU. To contaminate $5,000 \mathrm{lbs}$ of waste to TRU levels requires 0.23 curies of TRU.

Fortunately, the maximum concentration of plutonium in any SST is about $1.3 \times 10^{-5} \mathrm{Ci} / \mathrm{g}$ in Tank TX-118. About $538 \mathrm{~g}$ of this waste would be required to make $150 \mathrm{lbs}$ of waste TRU. About $18 \mathrm{~kg}$ of this waste would be required to make $5,000 \mathrm{lbs}$ of waste TRU. Only four other tanks have a significant potential for TRU contamination based on the TRU concentration in the tank and a significant volume of solids. A large volume of solids is required to contaminate more than the tip of the equipment. A small amount of contamination on the end of the equipment to cause a TRU designation. 
Eighteen other tanks have some potential for creating TRU waste based on the concentration of TRU in the solids and the amount of solids in the tanks. None of the five tanks with significant TRU solids are scheduled for retrieval through 2003. Only one of the eighteen tanks with some potential for TRU contamination is scheduled for retrieval through 2003. However, if equipment is pulled out of tanks three years before retrieval begins, a total of seven out of the 18 tanks are affected. The probability of very long, light-weight equipment in these tanks being TRU is estimated to be in the range of 10 percent to 50 percent. Based on this estimate some of the long-length equipment removed during the years 2001, 2002, and 2003 may be TRU and was included in the forecast.

Based on the experience with Tank 241-SY-102, which had equipment removed which was TRU and contact handled, one-half of the TRU equipment was classified as contact handled and one-half as remote handled. 
WHC-SD-WM-TI-777

Revision 0

This page intentionally left blank. 


\subsection{REFERENCES}

Agnew, S. F., 1996, Hanford Tank Chemical and Radionuclide Inventories: HDW Model Rev. 3, LA-UR-96-858, Los Alamos National Laboratory, Los Alamos, New Mexico.

DOE, 1995, Transuranic Waste Baseline Inventory Report (Revision 2), DOE/CAO-95-1121, U.S. Department of Energy, Richland, Washington.

DOE, 1996, Tank Waste Remediation System (TWRS) Remote Handled Transuranic Waste Volume Forecast, Letter, Jackson Kinzer to A. L. Trego, (April 11, 1996).

U.S. Department of Energy, Richland, Washington.

Duncan, D. R., et al, 1993, Characterization of Past and Present Solid Waste Streams from the Plutonium-Uranium Extraction Plant, WHC-EP-0646, Westinghouse Hanford Company, Richland, Washington.

Galbraith, J. D., 1996, Personal Communication, Westinghouse Hanford Company, Richland, Washington.

Penwell, D. L., 1996, Personal Communication, Westinghouse Hanford Company, Richland, Washington.

Roach, H. L., 1996, Disposal of Tank Farm Long-Length Contaminated Equipment: Alternative Options Study and Engineering Support Information, WHC-SD-WM-ES-265, Rev. 0-A, Westinghouse Hanford Company, Richland, Washington.

Slaathaug, E. J., 1996, Tank Waste Remediation System Optimized Processing Strategy, WHC-SD-WM-TI-0694, Rev. 0, Westinghouse Hanford Company, Richland, Washington.

Valero, O. J., et al., 1996, FY 1996 Solid Waste Integrated Life-Cycle Forecast Volume Summary Volume II, WHC-EP-0900, Westinghouse Hanford Company, Richland, Washington. 
WHC-SD-WM-TI-777

Revision 0

This page intentionally left blank. 
WHC-SD-WM-TI-777

Revision 0

\section{APPENDIX A}

\section{SOLID WASTE FORECAST}


WHC-SD-WM-TI-777

Revision 0

This page intentionally left blank. 
Waste Generator Waste Class $\frac{\text { WHC HLVP }}{\text { CH_LLW_I }}$ End Of Lifecycle: 2028

$$
\text { (in cubic meters) }
$$

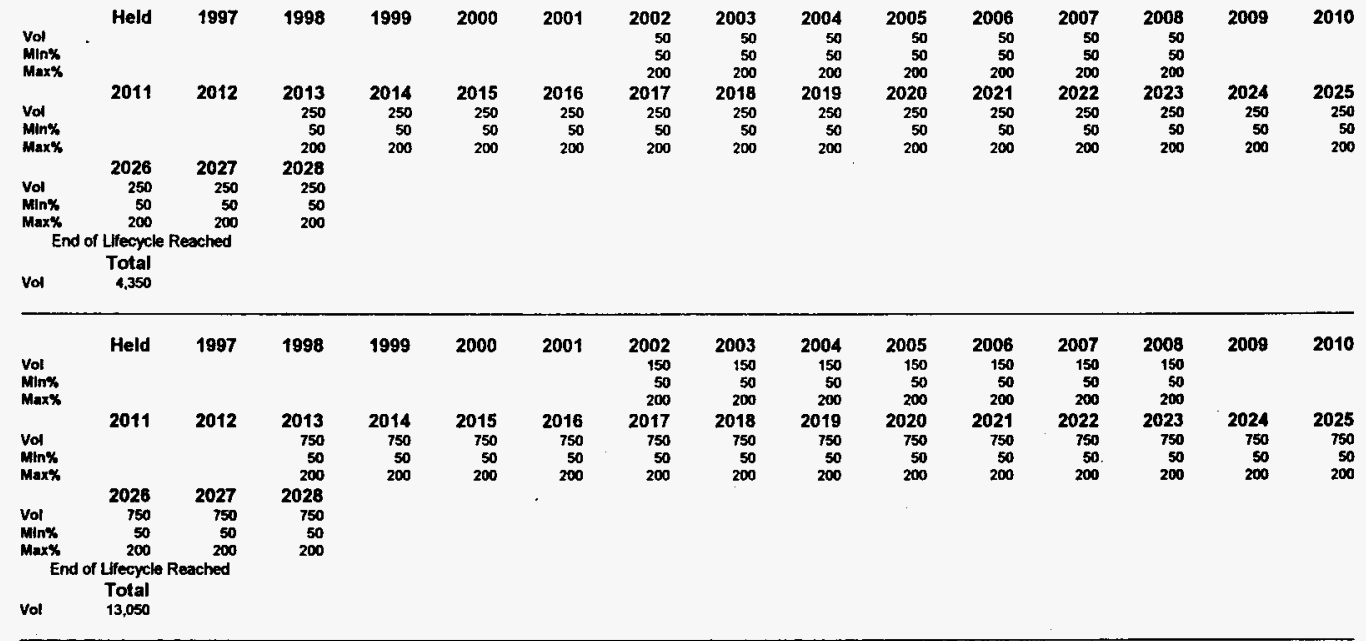


Waste Volumes and Percent Ranges (in cublc meters)

Waste Generator

$$
\text { Weste Class }
$$

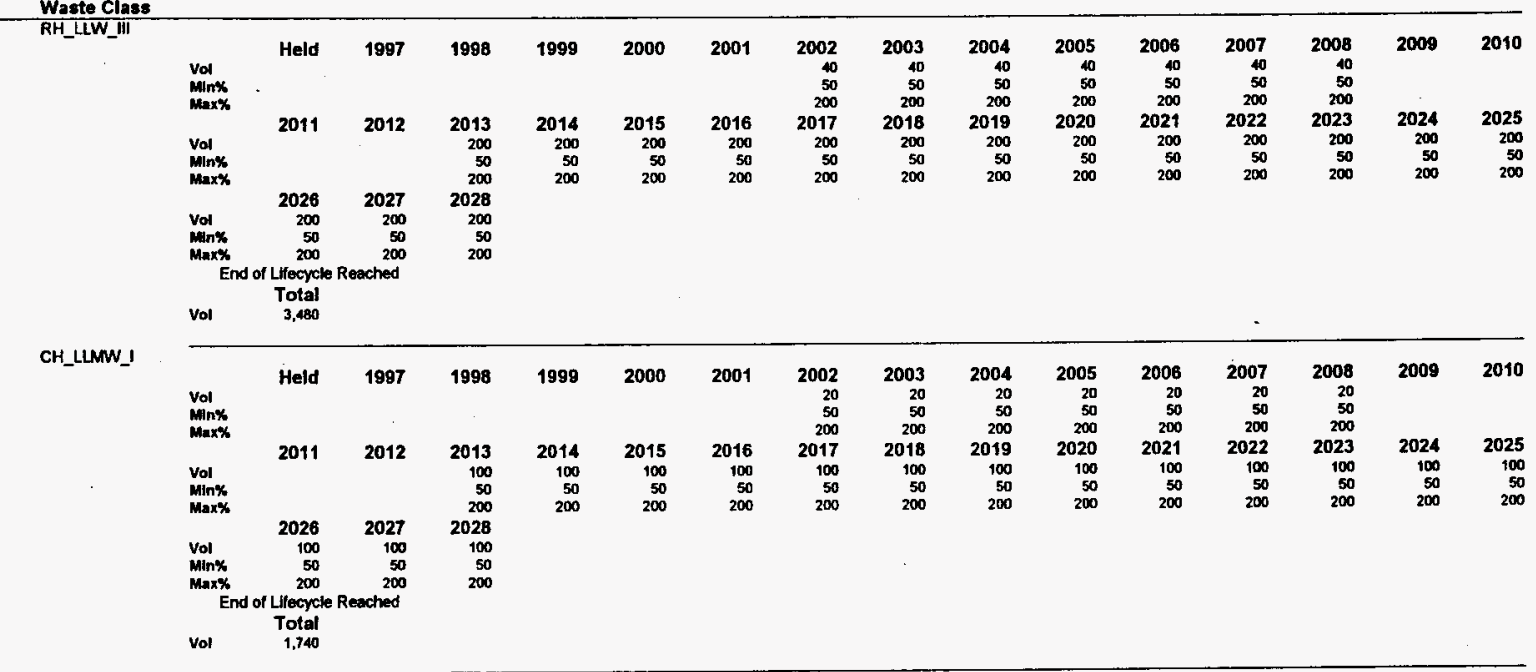


Waste Generator (in cubic meters)

\begin{tabular}{|c|c|c|c|c|c|c|c|c|c|c|c|c|c|c|c|}
\hline $\begin{array}{l}\text { Vol } \\
\min \% \\
\operatorname{Max} \%\end{array}$ & Held & 1997 & 1998 & 1999 & 2000 & 2001 & $\begin{array}{r}2002 \\
40 \\
50 \\
200\end{array}$ & $\begin{array}{r}2003 \\
40 \\
50 \\
200\end{array}$ & $\begin{array}{r}2004 \\
40 \\
50 \\
200\end{array}$ & $\begin{array}{r}2005 \\
40 \\
50 \\
200\end{array}$ & $\begin{array}{r}2006 \\
40 \\
50 \\
200\end{array}$ & $\begin{array}{r}2007 \\
40 \\
50 \\
200\end{array}$ & $\begin{array}{r}2008 \\
40 \\
50 \\
200\end{array}$ & 2009 & 2010 \\
\hline $\begin{array}{l}\text { Yol } \\
\text { Mlnx } \\
\text { Maxx }\end{array}$ & 2011 & 2012 & $\begin{array}{r}2013 \\
200 \\
50 \\
200\end{array}$ & $\begin{array}{r}2014 \\
200 \\
50 \\
200\end{array}$ & $\begin{array}{r}2015 \\
200 \\
50 \\
200\end{array}$ & $\begin{array}{r}2016 \\
200 \\
50 \\
200\end{array}$ & $\begin{array}{r}2017 \\
200 \\
50 \\
200\end{array}$ & $\begin{array}{r}2018 \\
200 \\
50 \\
200\end{array}$ & $\begin{array}{r}2019 \\
200 \\
50 \\
200\end{array}$ & $\begin{array}{r}2020 \\
200 \\
50 \\
200\end{array}$ & $\begin{array}{r}2021 \\
200 \\
50 \\
200\end{array}$ & $\begin{array}{r}2022 \\
200 \\
50 \\
200\end{array}$ & $\begin{array}{r}2023 \\
200 \\
50 \\
200\end{array}$ & $\begin{array}{r}2024 \\
200 \\
50 \\
200\end{array}$ & $\begin{array}{r}2025 \\
200 \\
50 \\
200\end{array}$ \\
\hline $\begin{array}{l}\text { Vol } \\
\text { Min* } \\
\text { Max* } \\
\text { En }\end{array}$ & $\begin{array}{r}2026 \\
200 \\
50 \\
200 \\
\text { L Lifecycle }\end{array}$ & $\begin{array}{r}2027 \\
200 \\
50 \\
200 \\
\text { ached }\end{array}$ & $\begin{array}{r}2028 \\
200 \\
50 \\
200\end{array}$ & & & & & & & & & & & & \\
\hline Vol & $\begin{array}{r}\text { Total } \\
3,480\end{array}$ & & & & & & & & & & & & & & \\
\hline
\end{tabular}

CH_TRUM

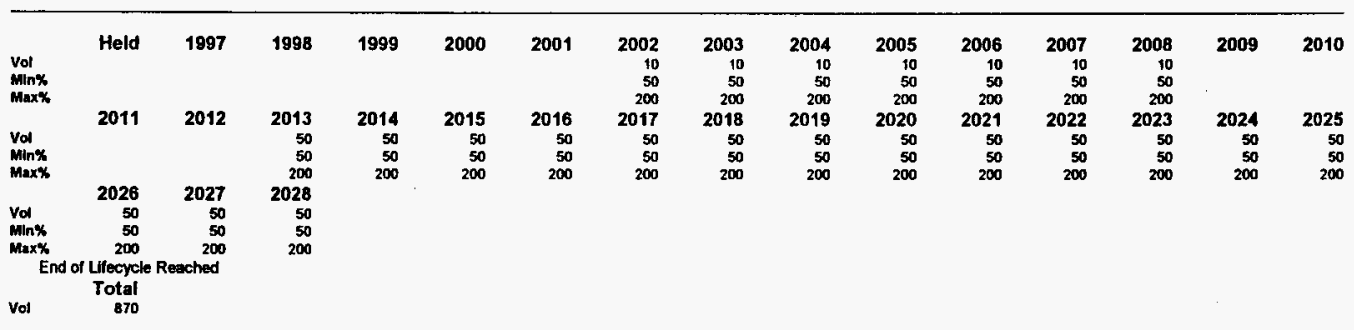



(in cubic meters)

Waste Generator

Waste Class

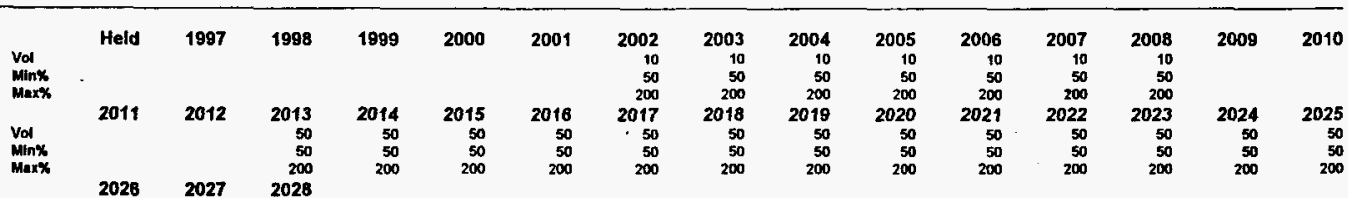

$\begin{array}{lrrr} & 2028 & 2027 & 2028 \\ \text { Vol } & 50 & 50 & 50 \\ \text { Min* } & 50 & 50 & 50\end{array}$

End of Llfecycte Reached

$$
\begin{gathered}
\text { Total } \\
870
\end{gathered}
$$

HAZ

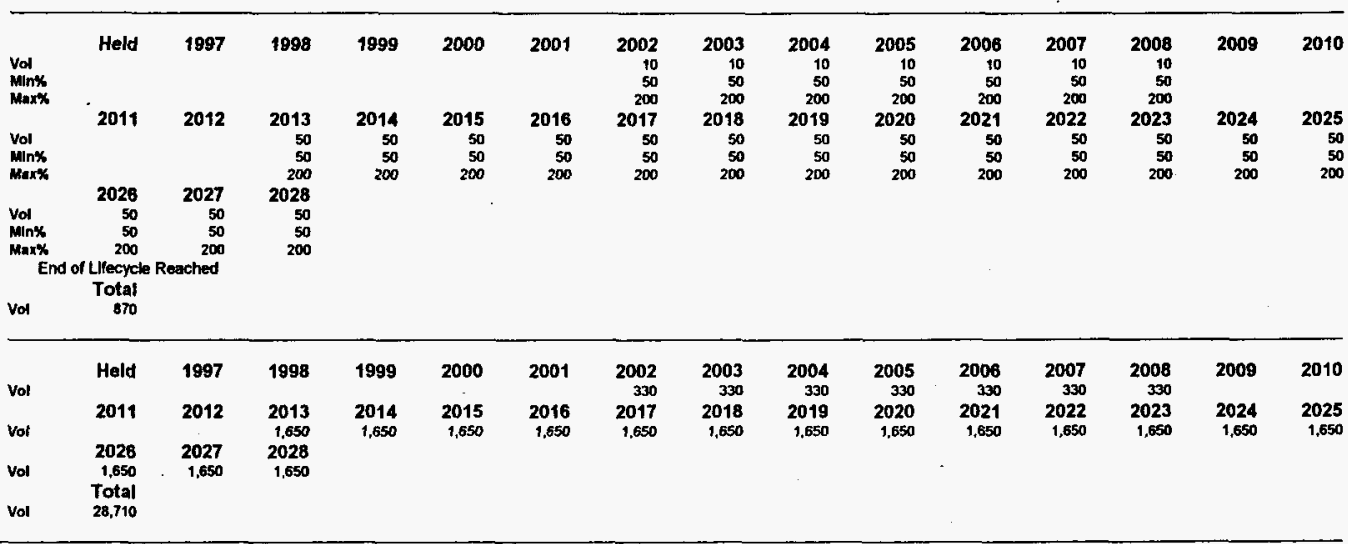



(in cublc meters)

Waste Generator

Waste Class

$\frac{\text { WHC LLVP }}{\text { CH ILW }}$

End Of Lifecycle: _.2021

\begin{tabular}{|c|c|c|c|c|c|c|c|c|c|c|c|c|c|c|c|}
\hline $\begin{array}{l}\text { Vol } \\
\text { Minx } \\
\text { Maxx }\end{array}$ & Held & 1997 & 1998 & 1999 & 2000 & 2001 & $\begin{array}{r}2002 \\
240 \\
50 \\
200\end{array}$ & $\begin{array}{r}2003 \\
240 \\
50 \\
200\end{array}$ & $\begin{array}{r}2004 \\
240 \\
50 \\
200\end{array}$ & $\begin{array}{r}2005 \\
240 \\
50 \\
200\end{array}$ & $\begin{array}{r}2006 \\
240 \\
50 \\
200\end{array}$ & $\begin{array}{r}2007 \\
240 \\
50 \\
200\end{array}$ & $\begin{array}{r}2008 \\
240 \\
50 \\
.200\end{array}$ & $\begin{array}{r}2009 \\
240 \\
50 \\
200\end{array}$ & $\begin{array}{r}2010 \\
240 \\
50 \\
200\end{array}$ \\
\hline & 2011 & 2012 & 2013 & 2014 & 2015 & 2016 & 2017 & 2018 & 2019 & 2020 & 2021 & & & & \\
\hline $\begin{array}{l}\text { Vol } \\
\text { Min } \\
\text { Max\% }\end{array}$ & $\begin{array}{r}1,200 \\
50 \\
200\end{array}$ & $\begin{array}{r}1,200 \\
50 \\
200\end{array}$ & $\begin{array}{r}1,200 \\
50 \\
200\end{array}$ & $\begin{array}{r}1,200 \\
50 \\
200\end{array}$ & $\begin{array}{r}1,200 \\
50 \\
200\end{array}$ & $\begin{array}{r}1,200 \\
50 \\
200\end{array}$ & $\begin{array}{r}1,200 \\
50 \\
200\end{array}$ & $\begin{array}{r}1,200 \\
50 \\
200\end{array}$ & $\begin{array}{r}1,200 \\
50 \\
200\end{array}$ & $\begin{array}{r}1,200 \\
50 \\
200\end{array}$ & $\begin{array}{r}1,200 \\
50 \\
200\end{array}$ & & & & \\
\hline Vol & $\begin{array}{l}\text { Lifecych } \\
\text { Total } \\
\$ 5,360\end{array}$ & lached & & & & & & & & & & & & & \\
\hline
\end{tabular}

CH_LLW_III

\begin{tabular}{|c|c|c|c|c|c|c|}
\hline $\begin{array}{l}\text { Vof } \\
\text { Min\% } \\
\text { Max\% }\end{array}$ & Held & 1997 & 1998 & 1999 & 2000 & 2001 \\
\hline & 2011 & 2012 & 2013 & 2014 & 2015 & 2016 \\
\hline $\begin{array}{l}\text { Vod } \\
\text { Minx } \\
\text { Max* }\end{array}$ & $\begin{array}{r}1,200 \\
50 \\
200\end{array}$ & $\begin{array}{r}1.200 \\
50 \\
200\end{array}$ & $\begin{array}{r}1,200 \\
50 \\
200\end{array}$ & $\begin{array}{r}1.200 \\
50 \\
200\end{array}$ & $\begin{array}{r}1,200 \\
50 \\
200\end{array}$ & $\begin{array}{r}1.200 \\
50 \\
200\end{array}$ \\
\hline Fo & $\begin{array}{l}\text { Lifecycle } \\
\text { Total }\end{array}$ & ached & & & & \\
\hline
\end{tabular}

RH LUW III

\begin{tabular}{|c|c|c|c|c|c|c|c|c|c|c|c|c|c|c|c|}
\hline $\begin{array}{l}\text { Yol } \\
\text { Min\% } \\
\text { Max\% }\end{array}$ & Held & 1997 & 1998 & 1999 & 2000 & 2001 & $\begin{array}{r}2002 \\
60 \\
50 \\
200\end{array}$ & $\begin{array}{r}2003 \\
60 \\
50 \\
200\end{array}$ & $\begin{array}{r}2004 \\
60 \\
50 \\
200\end{array}$ & $\begin{array}{r}2005 \\
60 \\
50 \\
200\end{array}$ & $\begin{array}{r}2006 \\
60 \\
50 \\
200\end{array}$ & $\begin{array}{r}2007 \\
60 \\
50 \\
200\end{array}$ & $\begin{array}{r}2008 \\
60 \\
50 \\
200\end{array}$ & $\begin{array}{r}2009 \\
60 \\
50 \\
200\end{array}$ & $\begin{array}{r}2010 \\
60 \\
50 \\
200\end{array}$ \\
\hline & 2011 & 2012 & 2013 & 2014 & 2015 & 2016 & 2017 & 2018 & 2019 & 2020 & 2021 & & & & \\
\hline $\begin{array}{l}\text { Vol } \\
\text { Min\% } \\
\text { Max\% }\end{array}$ & $\begin{array}{r}300 \\
50 \\
200\end{array}$ & $\begin{array}{r}300 \\
50 \\
200\end{array}$ & $\begin{array}{r}300 \\
50 \\
200\end{array}$ & $\begin{array}{r}300 \\
50 \\
200\end{array}$ & $\begin{array}{r}300 \\
50 \\
200\end{array}$ & $\begin{array}{r}300 \\
50 \\
200\end{array}$ & $\begin{array}{r}300 \\
50 \\
200\end{array}$ & $\begin{array}{r}300 \\
50 \\
200\end{array}$ & $\begin{array}{r}300 \\
50 \\
200\end{array}$ & $\begin{array}{r}300 \\
50 \\
200\end{array}$ & $\begin{array}{r}300 \\
50 \\
200\end{array}$ & & & & \\
\hline En & Lifecycle & ached & & & & & & & & & & & & & \\
\hline
\end{tabular}

\begin{tabular}{|c|c|c|c|c|c|c|c|c|}
\hline $\begin{array}{r}2002 \\
240 \\
50 \\
200 \\
2017 \\
1.200 \\
50 \\
200\end{array}$ & $\begin{array}{r}2003 \\
240 \\
50 \\
200 \\
2018 \\
1,200 \\
50 \\
200\end{array}$ & $\begin{array}{r}2004 \\
240 \\
50 \\
200 \\
2019 \\
1.200 \\
50 \\
200\end{array}$ & $\begin{array}{r}2005 \\
240 \\
50 \\
200 \\
2020 \\
1,200 \\
50 \\
200\end{array}$ & $\begin{array}{r}2006 \\
240 \\
50 \\
200 \\
2021 \\
1,200 \\
50 \\
200\end{array}$ & $\begin{array}{r}2007 \\
240 \\
50 \\
200\end{array}$ & $\begin{array}{r}2008 \\
240 \\
50 \\
200\end{array}$ & $\begin{array}{r}2009 \\
240 \\
50 \\
200\end{array}$ & $\begin{array}{r}2010 \\
240 \\
50 \\
200\end{array}$ \\
\hline $\begin{array}{r}2002 \\
60 \\
50 \\
200 \\
2017 \\
300 \\
50 \\
200\end{array}$ & $\begin{array}{r}2003 \\
60 \\
50 \\
200 \\
2018 \\
300 \\
50 \\
200\end{array}$ & $\begin{array}{r}2004 \\
60 \\
50 \\
200 \\
2019 \\
300 \\
50 \\
200\end{array}$ & $\begin{array}{r}2005 \\
60 \\
50 \\
200 \\
2020 \\
300 \\
50 \\
200\end{array}$ & $\begin{array}{r}2006 \\
60 \\
50 \\
200 \\
2021 \\
300 \\
50 \\
200\end{array}$ & $\begin{array}{r}2007 \\
60 \\
50 \\
200\end{array}$ & $\begin{array}{r}2008 \\
60 \\
50 \\
200\end{array}$ & $\begin{array}{r}2009 \\
60 \\
50 \\
200\end{array}$ & $\begin{array}{r}2010 \\
60 \\
50 \\
200\end{array}$ \\
\hline
\end{tabular}



(in cubic meters)

Waste Generator

Waste Class

CH_LIMW_!

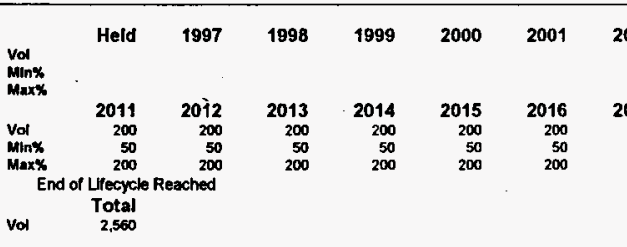

CH_LMW_III

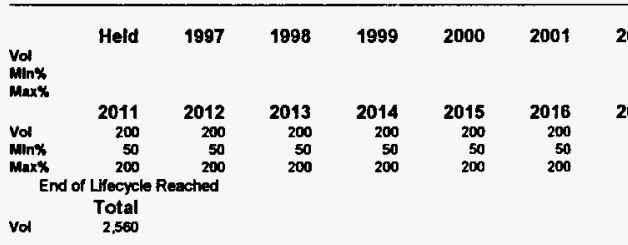

$\begin{array}{rrrrrrrrr}2002 & 2003 & 2004 & 2005 & 2006 & 2007 & 2008 & 2009 & 2010 \\ 40 & 40 & 40 & 40 & 40 & 40 & 40 & 40 & 40 \\ 50 & 50 & 50 & 50 & 50 & 50 & 50 & 50 & 50 \\ 200 & 200 & 200 & 200 & 200 & 200 & 200 & 200 & 200 \\ 2017 & 2018 & 2019 & 2020 & 2021 & & & & \end{array}$

HAZ

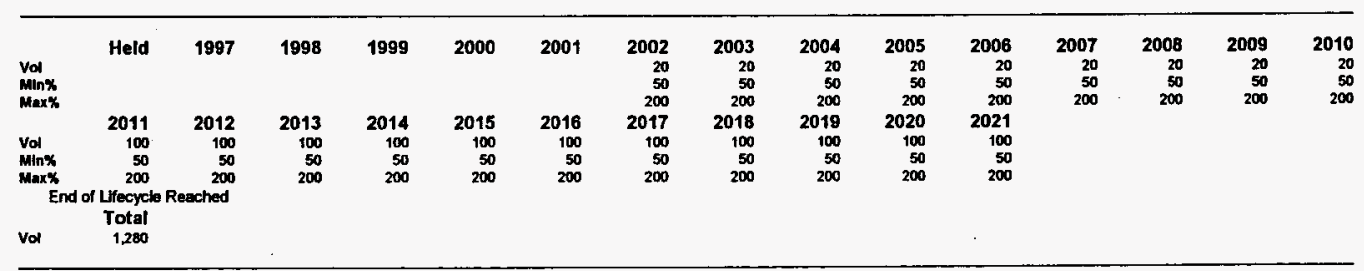


WHC-SD-WM-TI-777

Revision 0

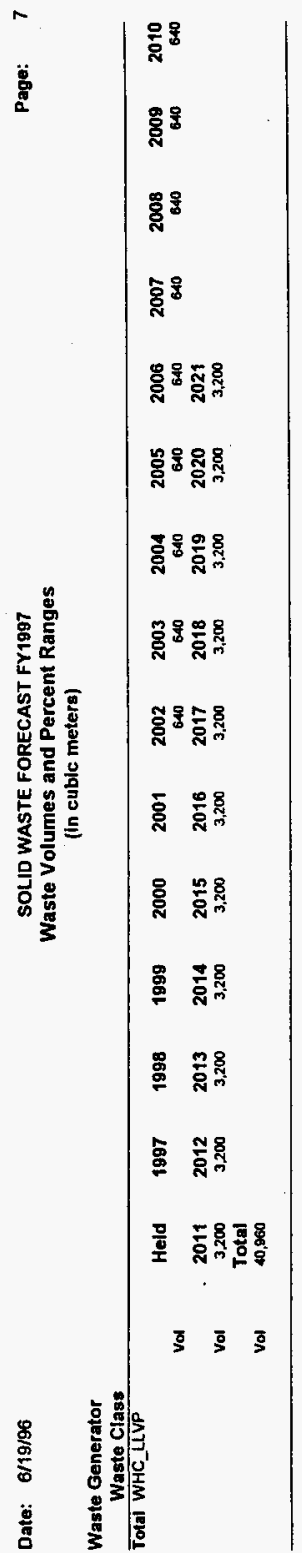


Waste Generator

(in cubic meters)

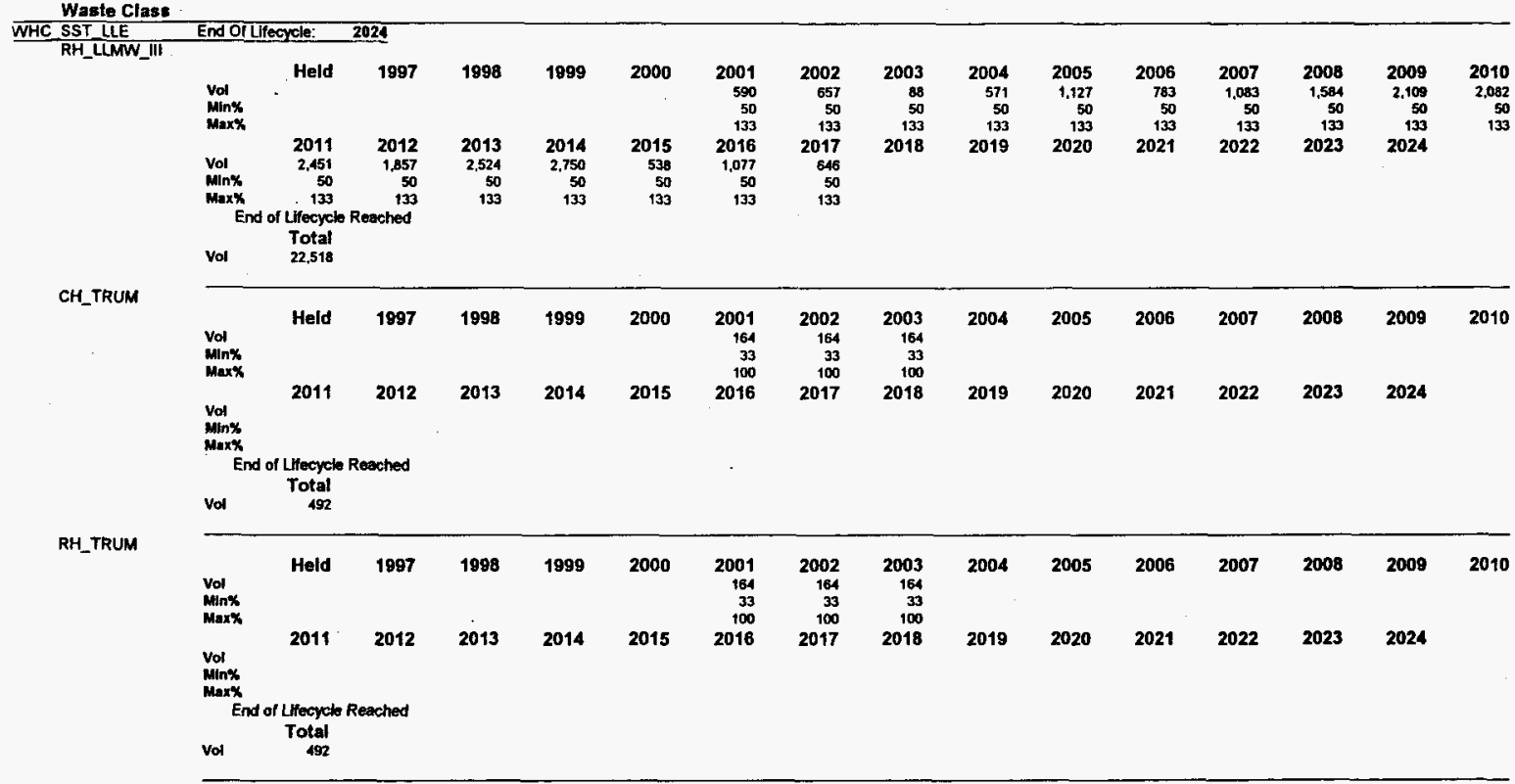

$\frac{7}{0}$ 
Waste Generator

$$
\text { Waste Class }
$$

Total WHC_SST_LLE

\begin{tabular}{|c|c|c|c|c|c|c|c|c|c|c|c|c|c|c|c|}
\hline Vot & & & & & & $\begin{array}{r}2001 \\
917\end{array}$ & $\begin{array}{r}2002 \\
985\end{array}$ & $\begin{array}{r}2003 \\
416\end{array}$ & $\begin{array}{r}2004 \\
571\end{array}$ & $\begin{array}{r}2005 \\
\mathbf{1 , 1 2 7}\end{array}$ & $\begin{array}{r}2006 \\
789\end{array}$ & $\begin{array}{r}2007 \\
1,0033\end{array}$ & $\begin{array}{r}2008 \\
1,584\end{array}$ & $\begin{array}{r}2009 \\
2,109\end{array}$ & $\begin{array}{r}2010 \\
2.082\end{array}$ \\
\hline & - 2011 & 2012 & 2013 & 2014 & 2015 & 2016 & 2017 & 2018 & 2019 & 2020 & 2021 & 2022 & 2023 & 2024 & \\
\hline Vol & $\begin{array}{r}2.451 \\
\text { Total }\end{array}$ & 1,057 & 2,524 & 2.750 & 538 & 1,077 & 648 & & & & & & & & \\
\hline Vol & 23,501 & & & & & & & & & & & & & & \\
\hline
\end{tabular}

$$
\text { umes and Percent Ranges }
$$

$$
\text { (in cubic meters) }
$$


Waste Generator

Waste Clas:

$\frac{\text { WHC SST RET }}{\text { CH_LLW_I }}$ End Of Lifecycle: $\quad \mathbf{2 0 1 8}$

$$
\text { (in cubic meters) }
$$

\begin{tabular}{|c|c|c|c|c|c|c|c|c|c|c|c|c|c|c|c|}
\hline $\begin{array}{l}\text { Vol } \\
\text { Mins } \\
\text { Max* }\end{array}$ & 2011 & 1997 & 1998 & 1999 & 2015 & $\begin{array}{l}2001 \\
2016\end{array}$ & $\begin{array}{l}2002 \\
2017\end{array}$ & $\begin{array}{l}2003 \\
2018\end{array}$ & $\begin{array}{r}2004 \\
16 \\
50 \\
200\end{array}$ & $\begin{array}{r}2005 \\
21 \\
50 \\
200\end{array}$ & $\begin{array}{r}2006 \\
4 \\
50 \\
200\end{array}$ & $\begin{array}{r}2007 \\
12 \\
50 \\
200\end{array}$ & $\begin{array}{r}2008 \\
33 \\
50 \\
200\end{array}$ & $\begin{array}{r}2009 \\
28 \\
50 \\
200\end{array}$ & $\begin{array}{r}2010 \\
36 \\
50 \\
200\end{array}$ \\
\hline
\end{tabular}

$\begin{array}{lrrrrrrr} & 2011 & 2012 & 2013 & 2014 & 2015 & 2016 & 2017 \\ \text { Vol } & 50 & 60 & 61 & 50 & 41 & 86 & 65 \\ \text { Minx } & 50 & 50 & 50 & 50 & 50 & 50 & 50 \\ \text { Max* } & 200 & 200 & 200 & 200 & 200 & 200 & 200 \\ \text { End of Llecycle Reached } & & & & & \end{array}$

Vol

CH_LUMW_I

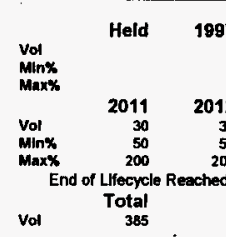

CH_LLMN_III

\begin{tabular}{|c|c|c|c|c|c|c|c|c|c|c|c|c|c|c|c|}
\hline $\begin{array}{l}\text { Vol } \\
\text { Min\% } \\
\text { Maxx }\end{array}$ & Held & 1997 & 1998 & 1999 & 2000 & 2001 & 2002 & 2003 & $\begin{array}{r}2004 \\
14 \\
50 \\
200\end{array}$ & $\begin{array}{r}2005 \\
17 \\
50 \\
200\end{array}$ & $\begin{array}{r}2006 \\
4 \\
50 \\
200\end{array}$ & $\begin{array}{r}2007 \\
10 \\
50 \\
200\end{array}$ & $\begin{array}{r}2008 \\
27 \\
50 \\
200\end{array}$ & $\begin{array}{r}2009 \\
24 \\
50 \\
200\end{array}$ & $\begin{array}{r}2010 \\
31 \\
50 \\
200\end{array}$ \\
\hline & 2011 & 2012 & 2013 & 2014 & 2015 & 2016 & 2017 & 2018 & & & & & & & \\
\hline $\begin{array}{l}\text { Vol } \\
\text { Mink } \\
\text { Maxx }\end{array}$ & $\begin{array}{r}40 \\
50 \\
200\end{array}$ & $\begin{array}{r}52 \\
50 \\
200\end{array}$ & $\begin{array}{r}51 \\
50 \\
200\end{array}$ & $\begin{array}{r}76 \\
50 \\
200\end{array}$ & $\begin{array}{r}34 \\
50 \\
200\end{array}$ & $\begin{array}{r}72 \\
50 \\
200\end{array}$ & $\begin{array}{r}55 \\
50 \\
200\end{array}$ & & & & & & & & \\
\hline & LIfecycle & ached & & & & & & & & & & & & & \\
\hline Vol & $\begin{array}{r}\text { Total } \\
50 ?\end{array}$ & & & & & & & & & & & & & & \\
\hline
\end{tabular}


Waste Volumes and Percent Ranges (in cubic meters)

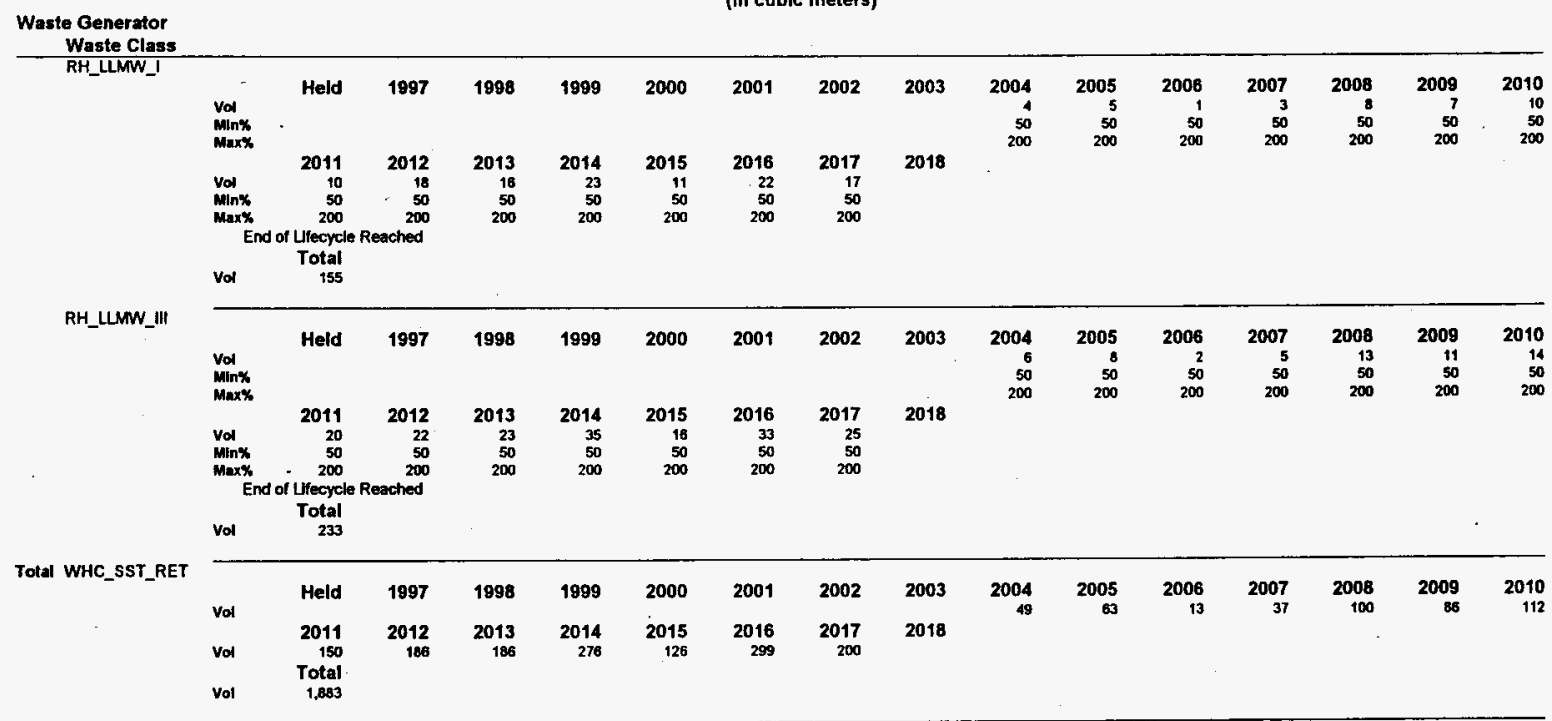

$\stackrel{3}{\stackrel{1}{2}}$ 
Waste Generator

Waste Class, Contelner

$\frac{\text { WHC HLVP }}{\text { CH_LLW_I, H-2-42701 (4x4x8) }}$

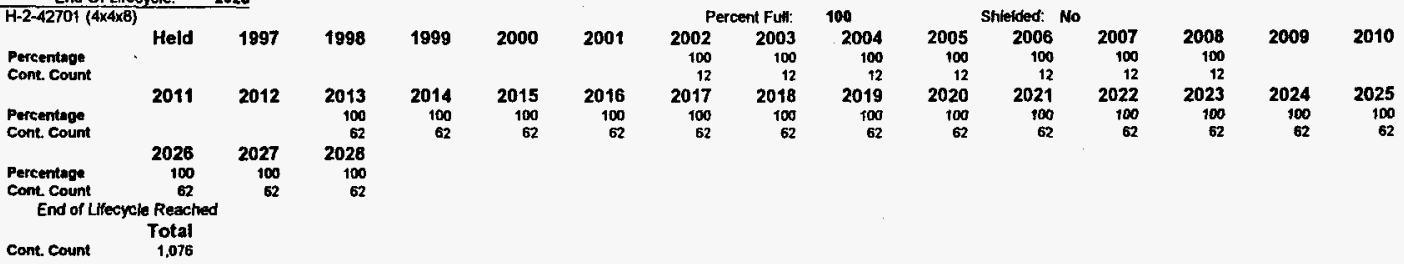

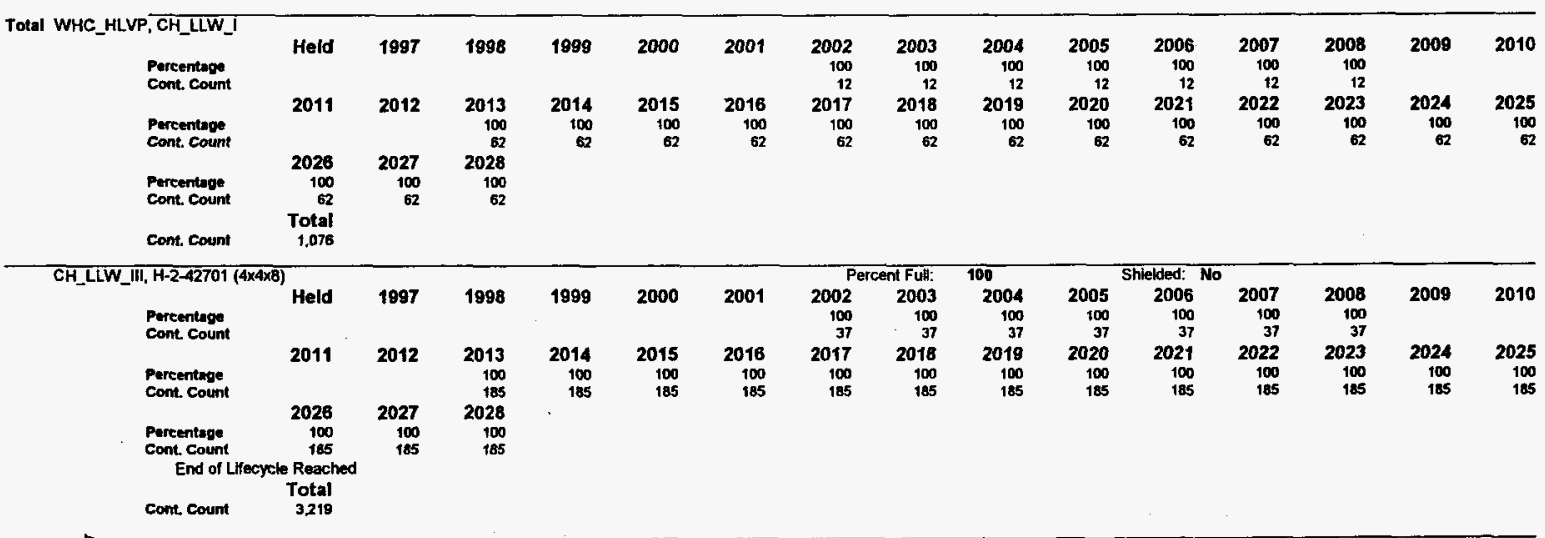

$\stackrel{7}{\frac{1}{7}}$ 


\section{Waste Generator}

Waste Class, Container

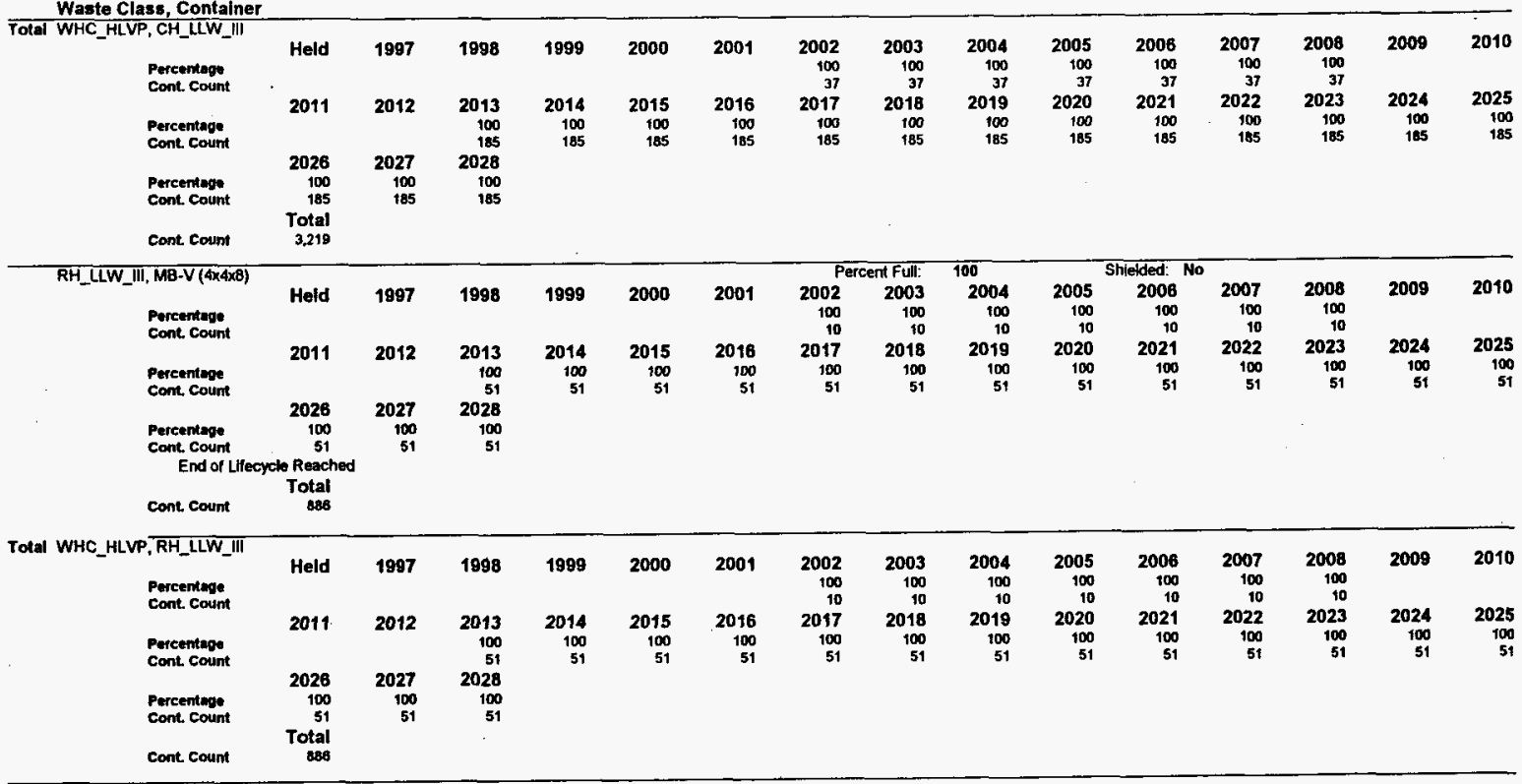


Waste Generator

Waste Class Container

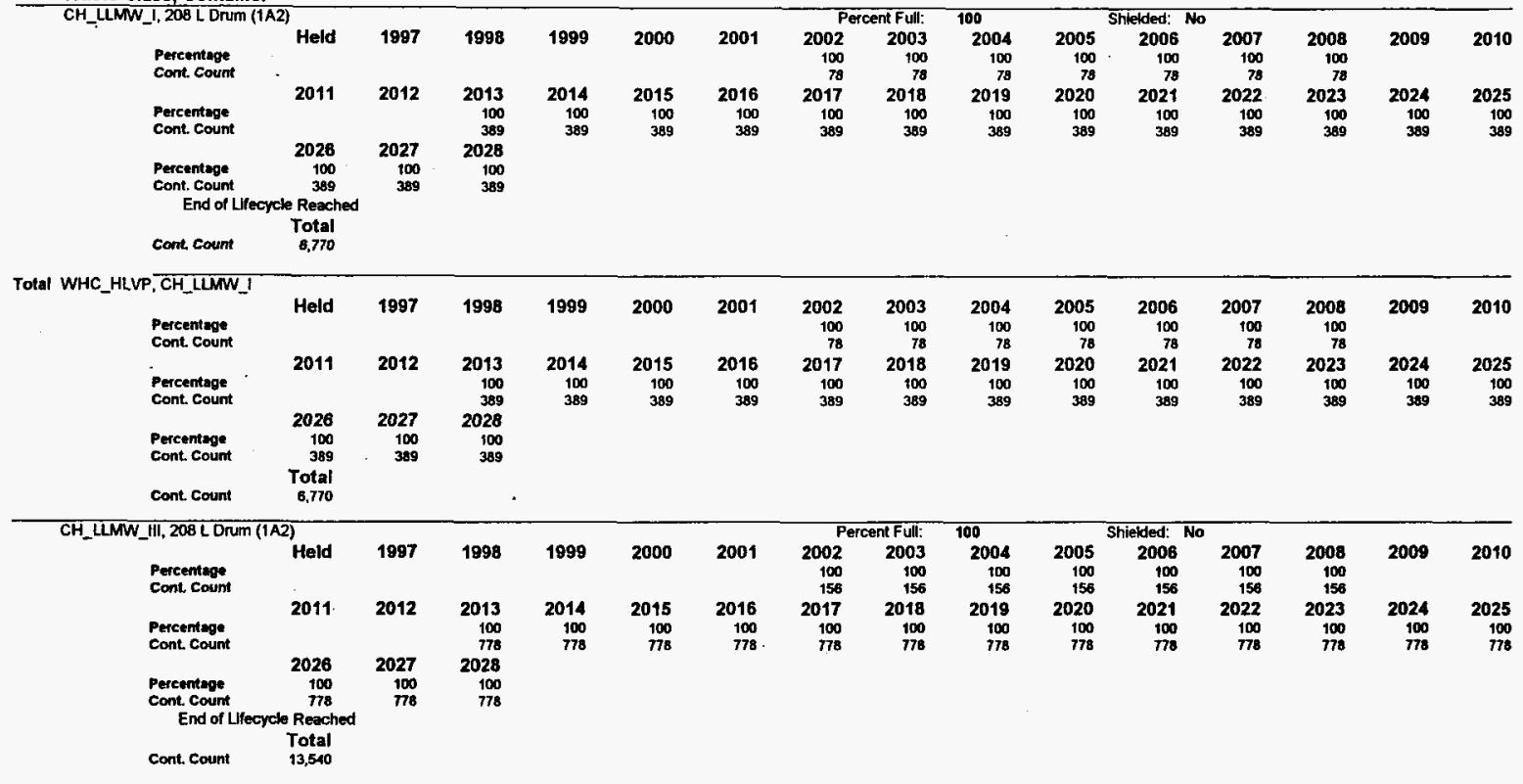

$\frac{7}{2}$ 
Waste Generator

Waste Class, Container

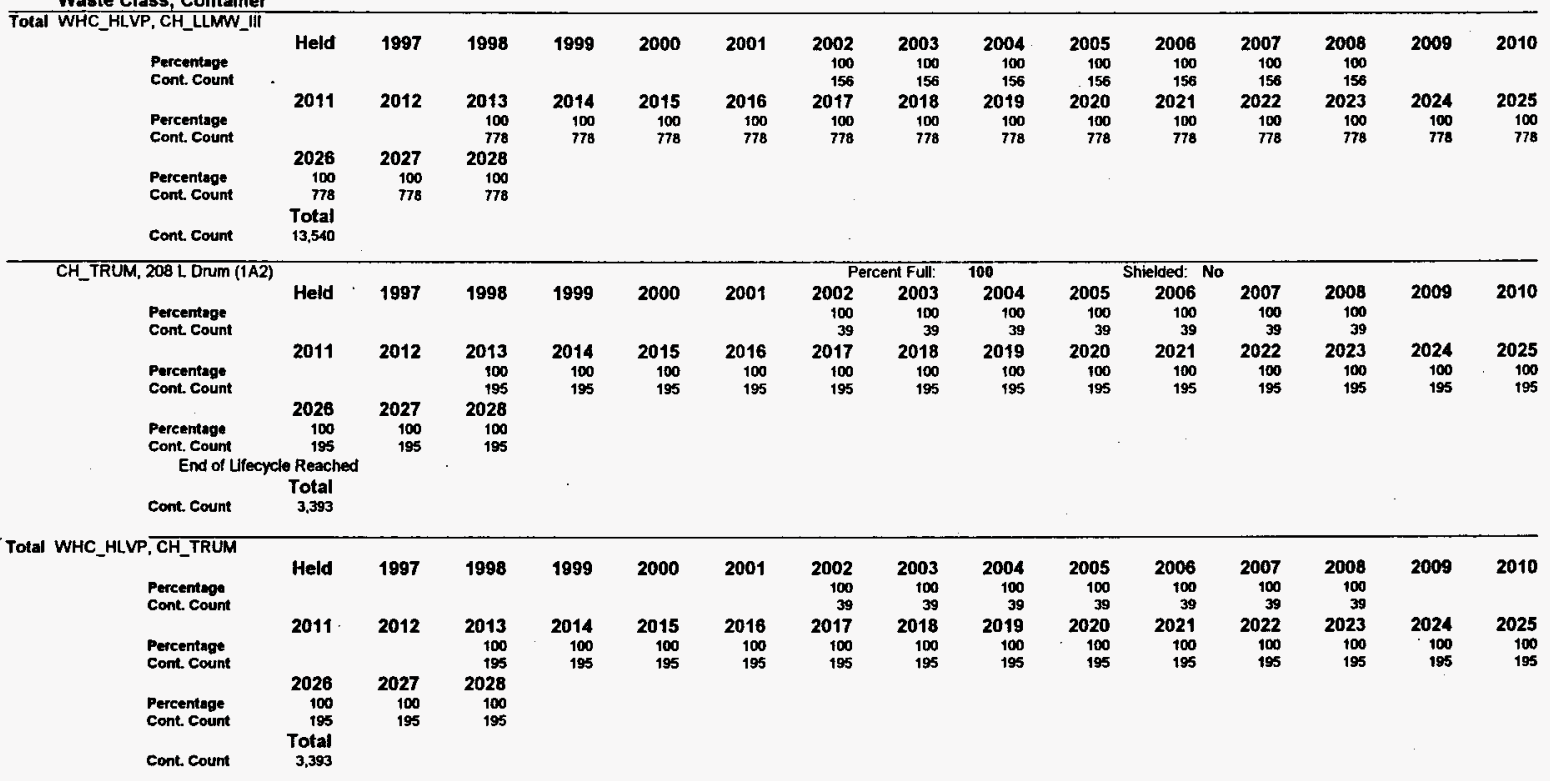


Waste Generator

Waste Class, Container

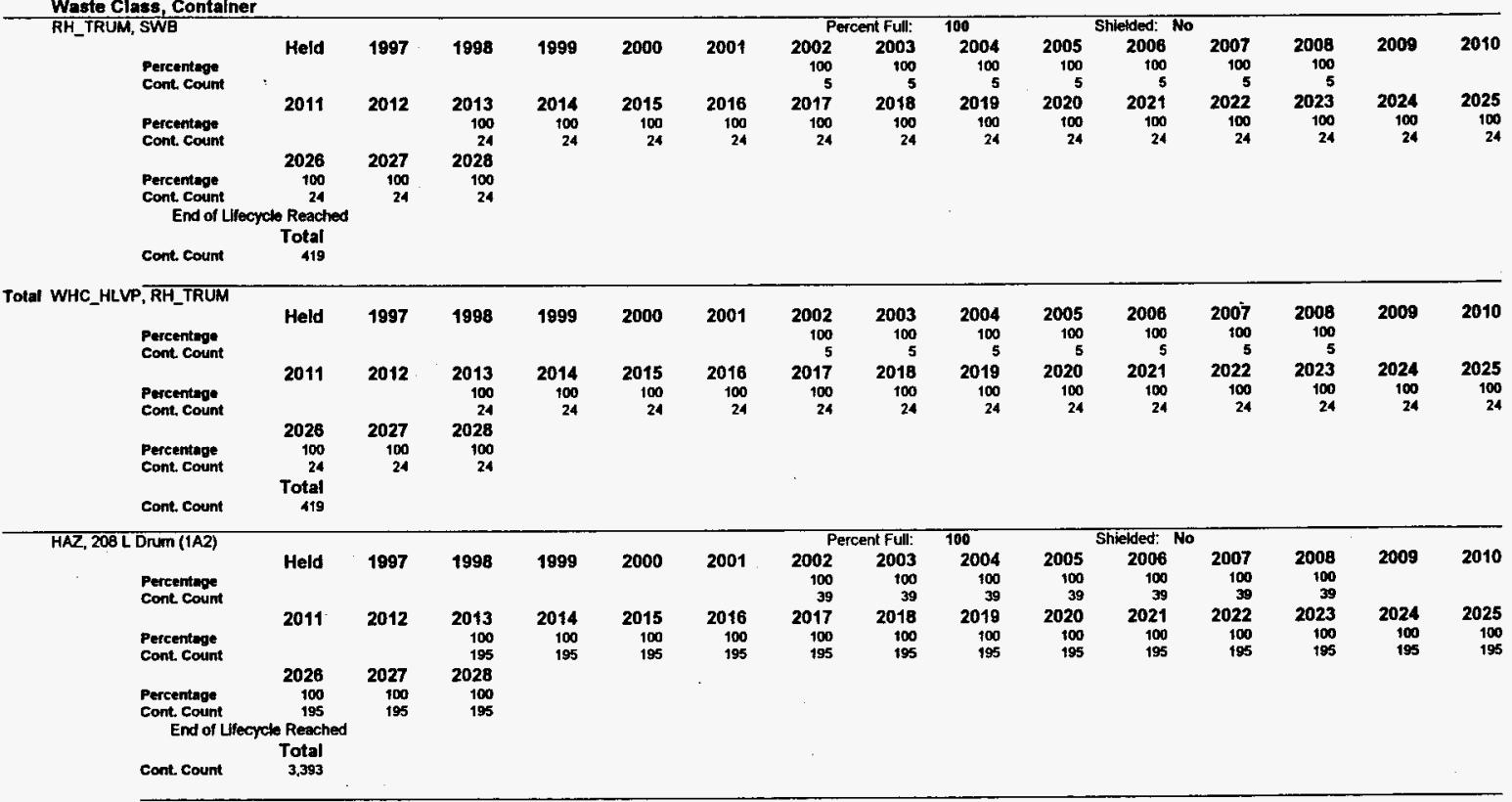

Container Types 


\section{Waste Generator}

Waste Class, Container

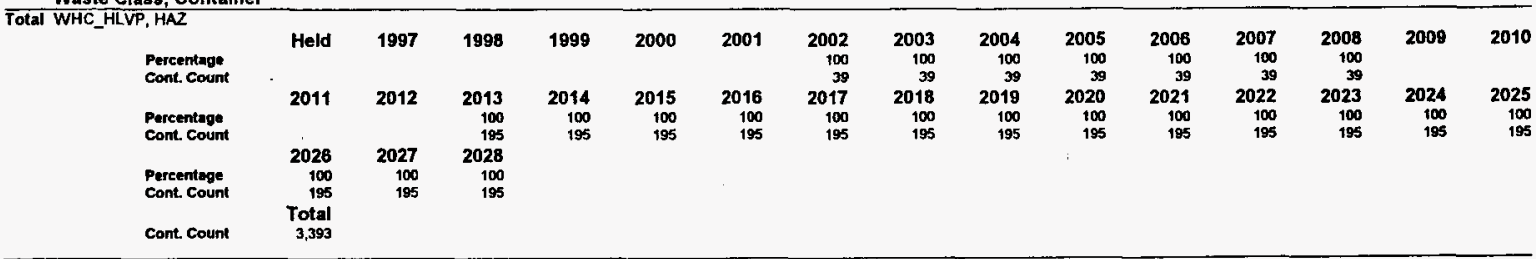


Waste Generator

Waste Class, Container

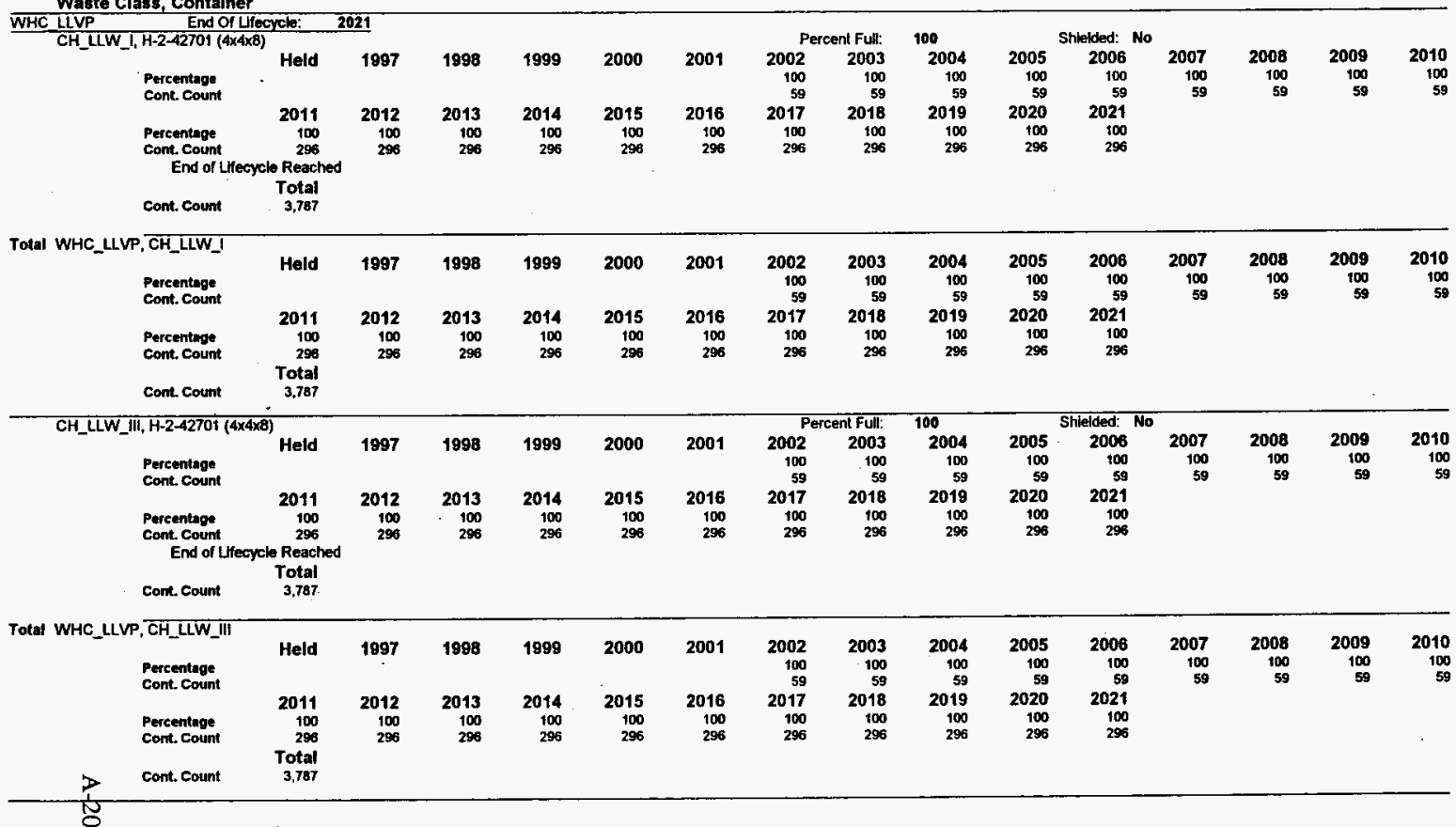


Waste Generator

Waste Class, Container

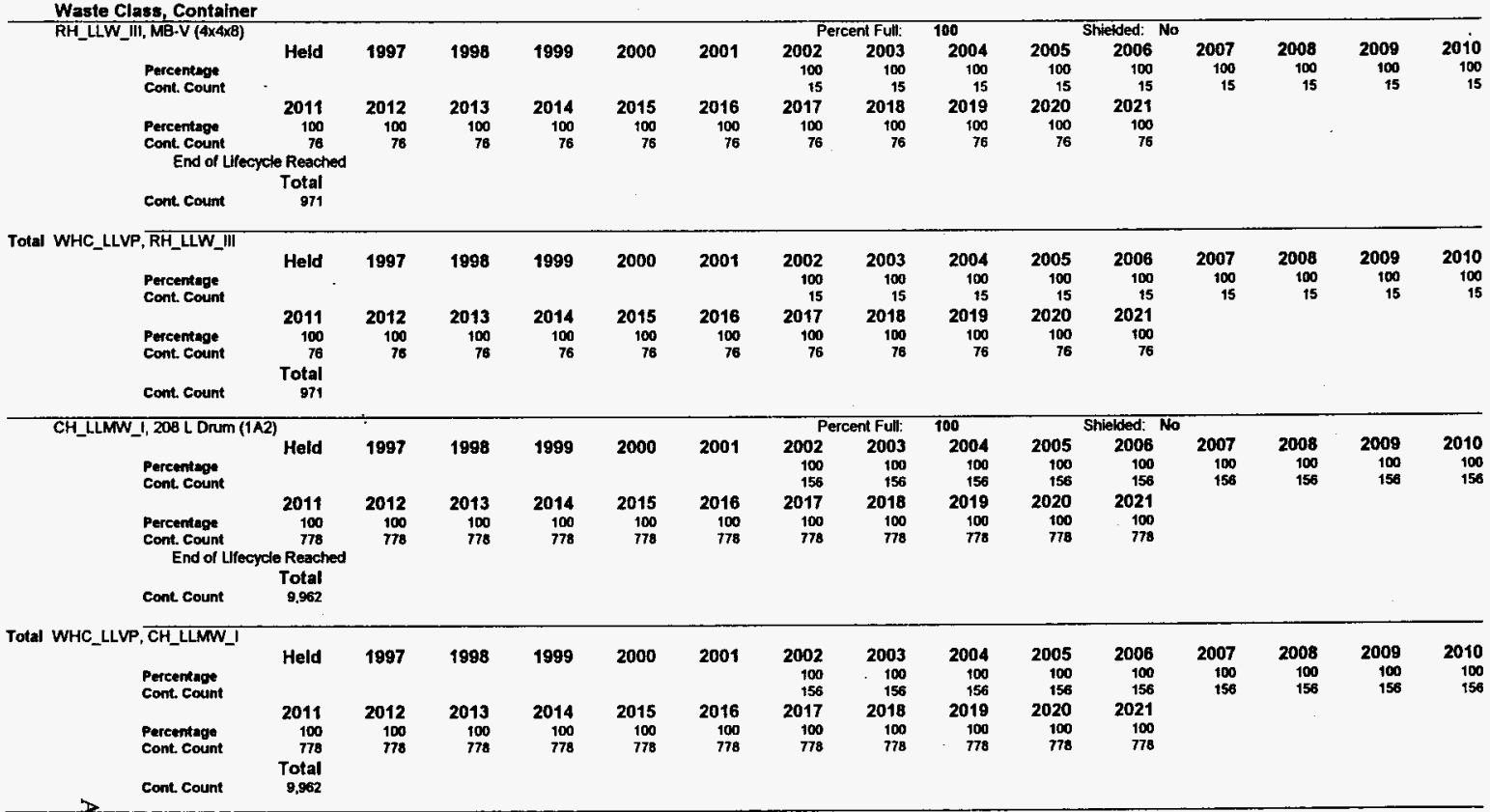

Container Types 
Waste Generator

Container

Waste Class, Container

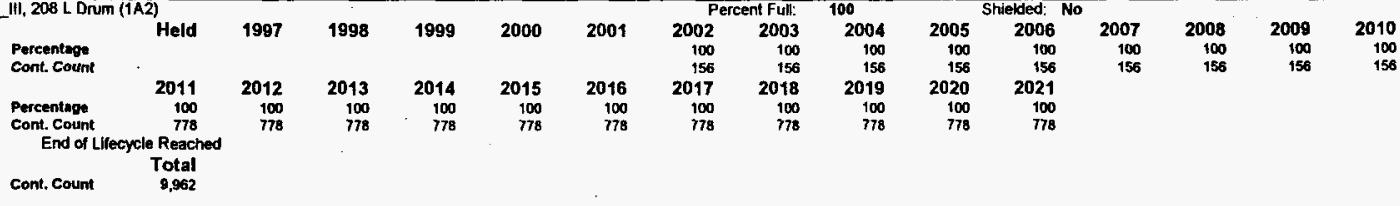

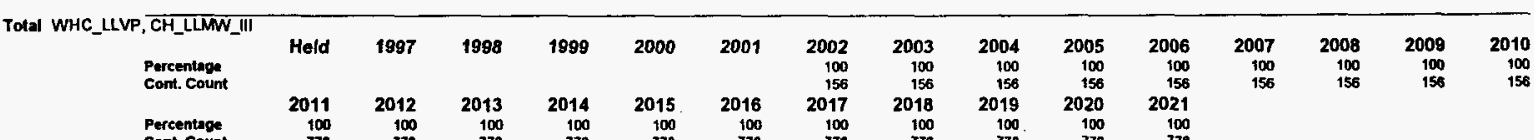

$\begin{array}{rrrrrrrrrrrr} & 2011 & 2012 & 2013 & 2014 & 2015 & 2016 & 2017 & 2018 & 2019 & 2020 & 2021 \\ \text { Percentage } & 100 & 100 & 100 & 100 & 100 & 100 & 100 & 100 & 100 & 100 & 100 \\ \text { Cont Count } & 778 & 778 & 778 & 778 & 778 & 778 & 778 & 778 & 778 & 778 & 778\end{array}$

com. count

Total

Total

HAZ, 208 L DNUm (1A2)

Held $1997 \quad 1998 \quad 1999 \quad 2000 \quad 2001 \quad$ Percent Full: 100

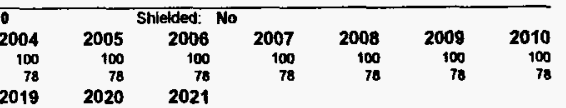

Pertentage
Cont Coumt

Percentege

$\begin{array}{lllllll}2011 & 2012 & 2013 & 2014 & 2015 & 2016 & 2001\end{array}$

$\begin{array}{rrrrr}2002 & 2003 & 2004 & 2005 & 2006 \\ 100 & 100 & 100 & 100 & \\ 78 & 78 & 78 & 78 & \\ 2017 & 2018 & 2019 & 2020 & 202 \\ 100 & 100 & 100 & 100 & \\ 389 & 389 & 389 & 389 & \end{array}$

End of Lffecycle Reached

Total

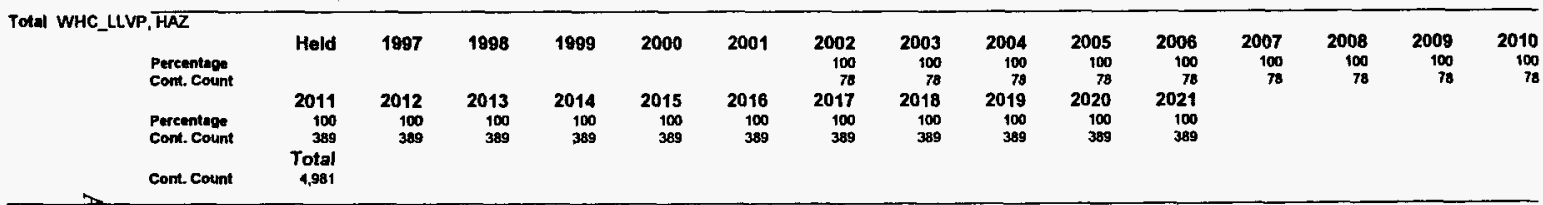


Waste Generator

Waste Class Container

WHC SST LLE

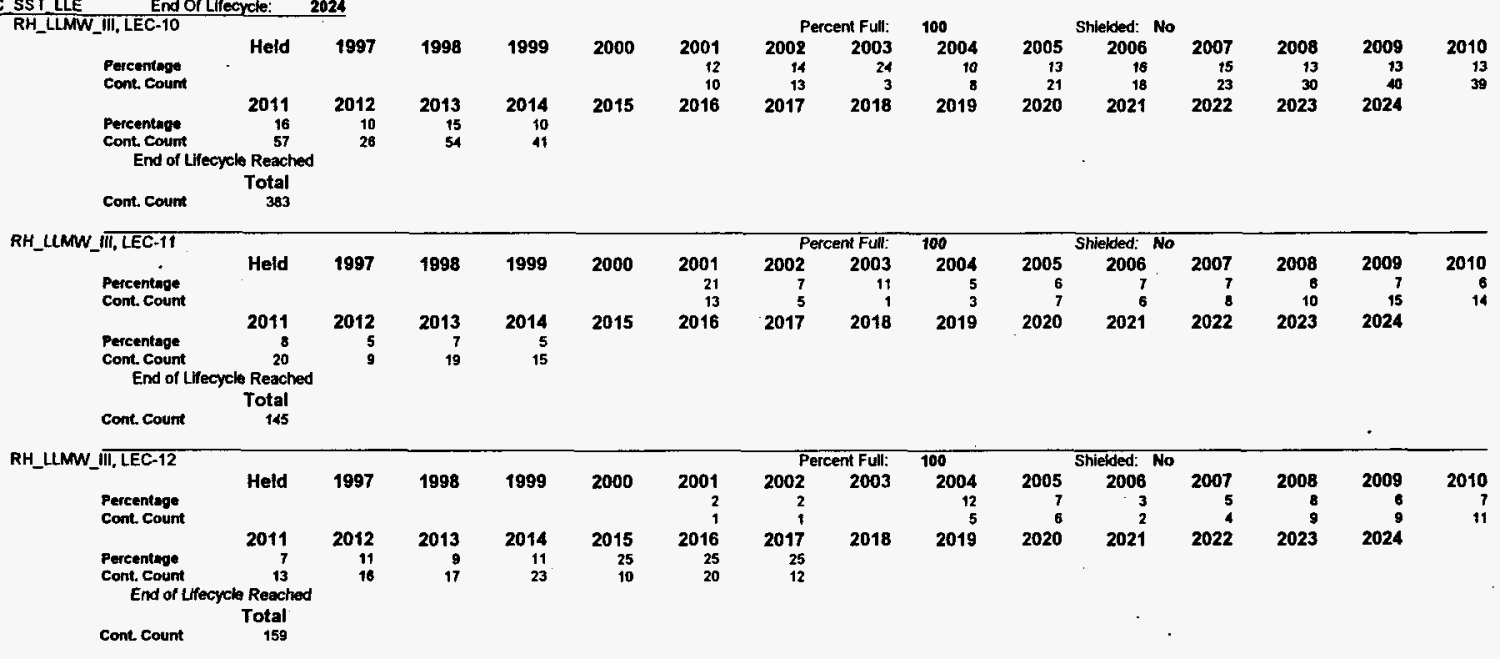




\section{Waste Generator}

Waste Class, Container

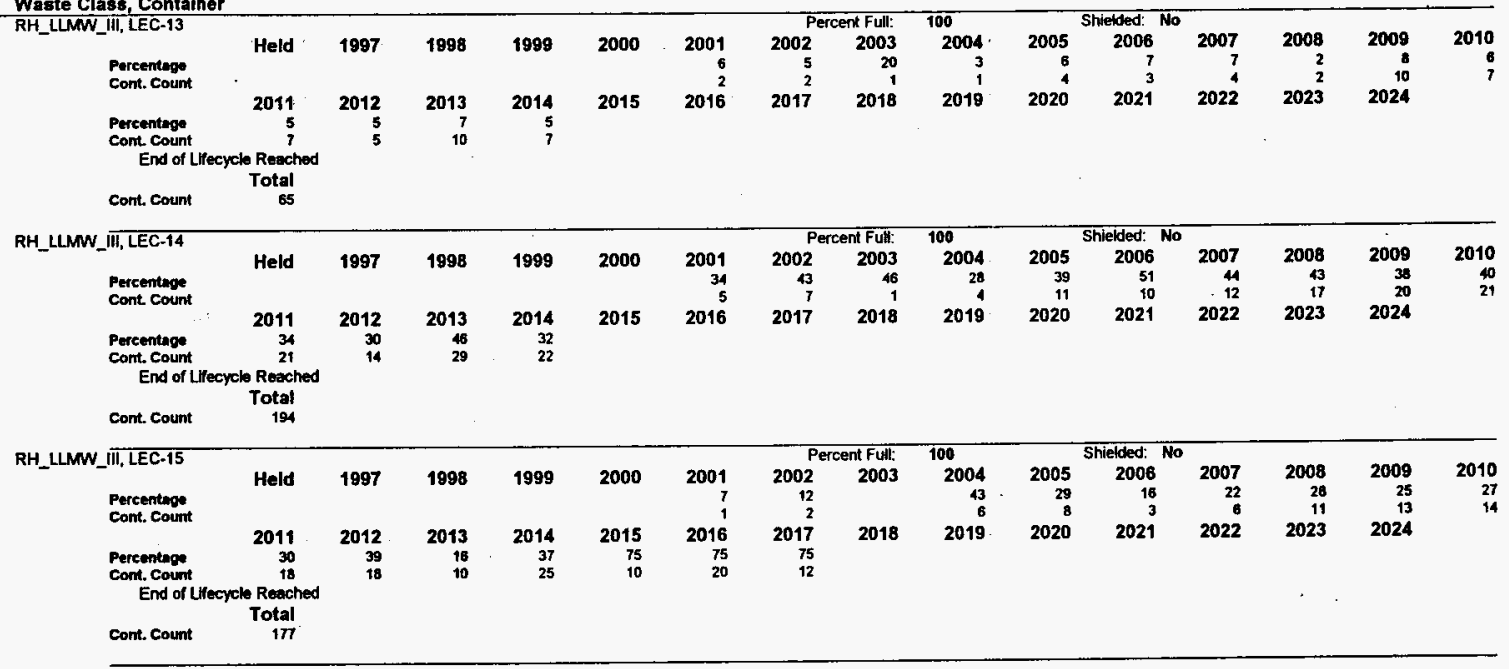


Waste Generator

Waste Class, Container

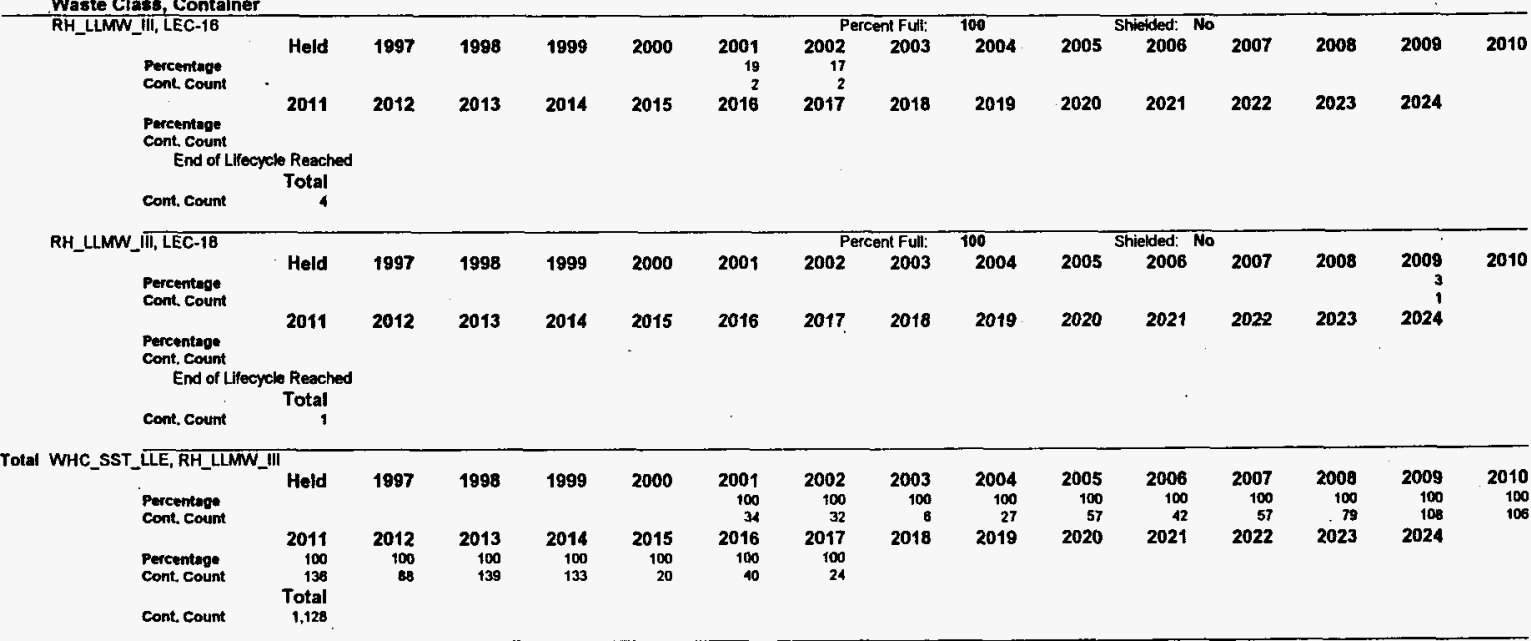


Waste Generator

Waste Class, Container

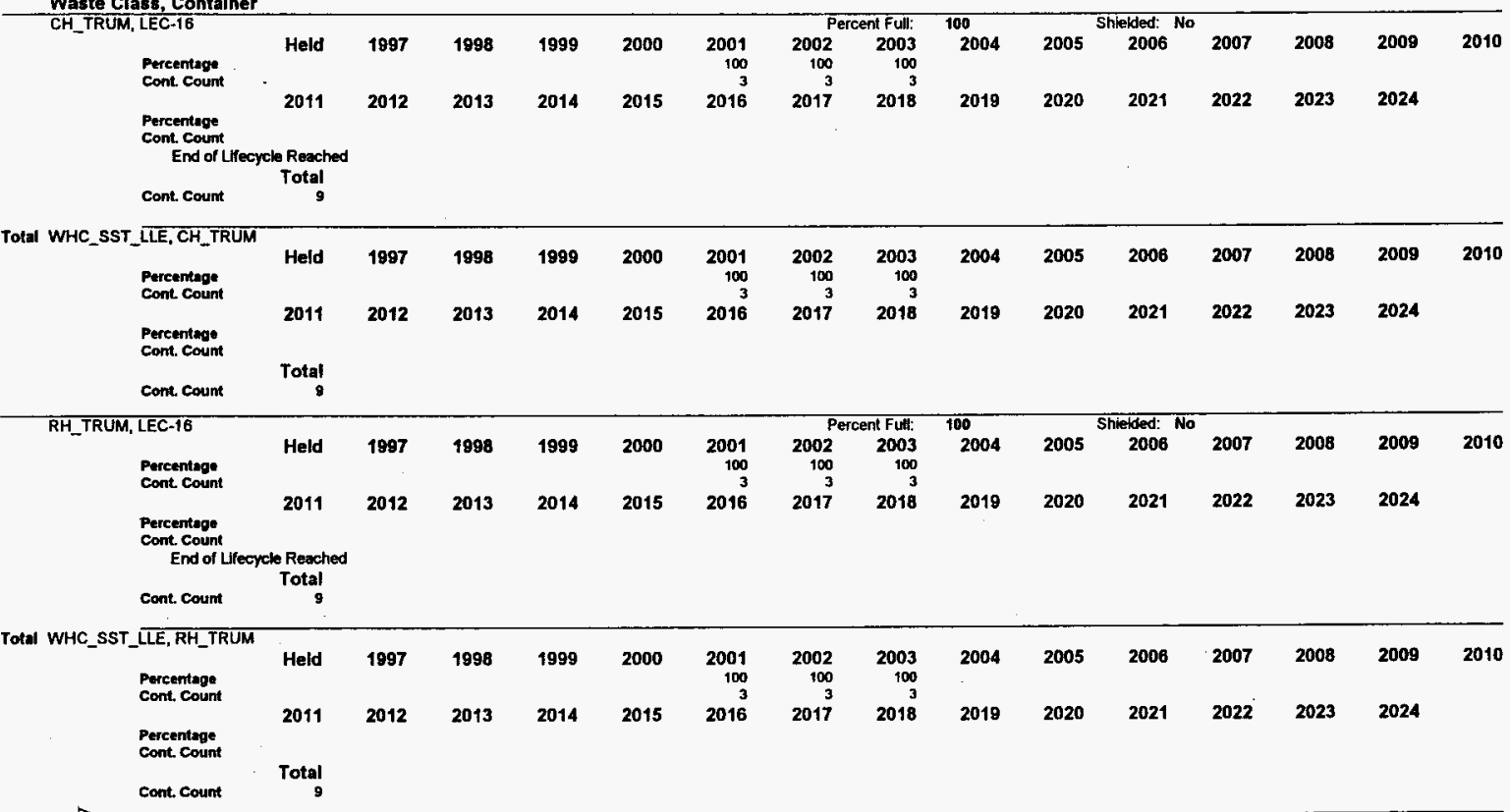


Container Types

Waste Generator

Waste Class, Contalner

End Of Lilecyc

CH_LLW_i, 208 L Drum (1A1)

\begin{tabular}{|c|c|c|c|c|c|c|c|c|c|c|c|c|c|c|c|}
\hline 208 L Drum (1A1) & & & & & & & & nt Ful: & 100 & & Shleided: No & & & & \\
\hline \multirow{2}{*}{$\begin{array}{l}\text { Percentage } \\
\text { Cont. Count }\end{array}$} & Held & 1997 & 1998 & 1999 & 2000 & 2001 & 2002 & 2003 & $\begin{array}{r}2004 \\
100\end{array}$ & $\begin{array}{r}2005 \\
100\end{array}$ & $\begin{array}{r}2006 \\
100\end{array}$ & $\begin{array}{r}2007 \\
100\end{array}$ & $\begin{array}{r}2008 \\
100\end{array}$ & $\begin{array}{r}2009 \\
100\end{array}$ & $\begin{array}{r}2010 \\
100\end{array}$ \\
\hline & 2011 & 2012 & 2013 & 2014 & 2015 & 2016 & 2017 & 2018 & & & & & & & 148 \\
\hline
\end{tabular}

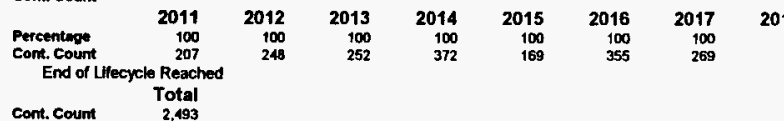

cont. Count 2,493

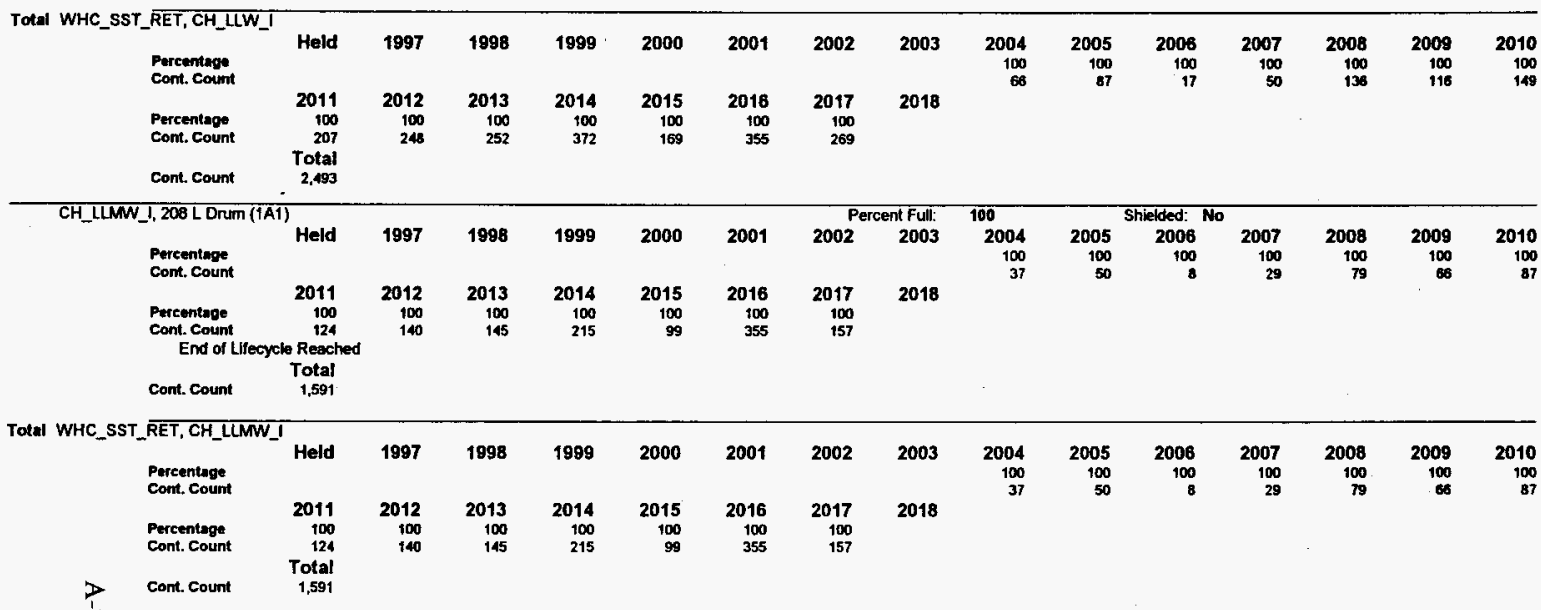


Waste Generator

Waste Class, Container

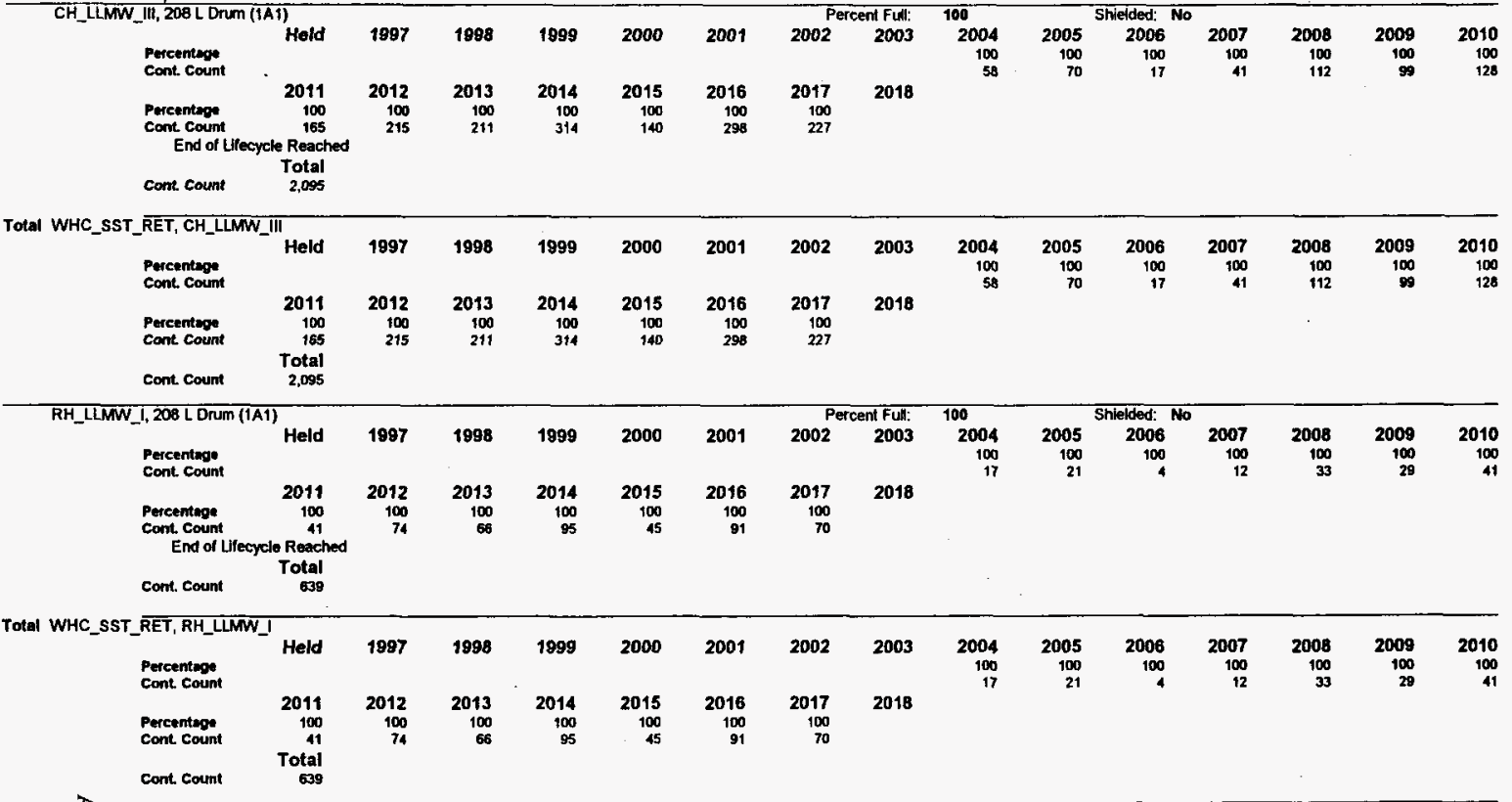



Container Types

\section{Waste Generator}

Waste Class, Container

RH LIMW III, 20 S L Drumer

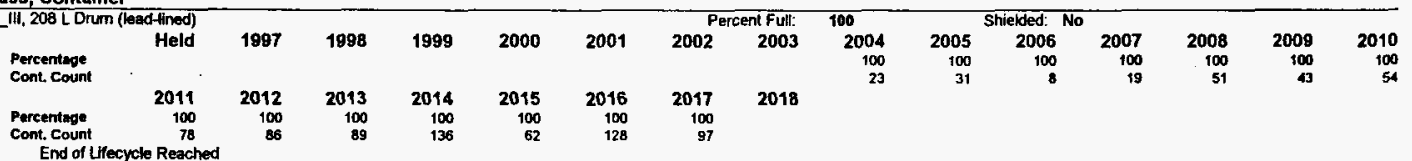

End of Lifecycle Reached

$\begin{array}{rr} & \text { Total } \\ \text { cont. Count } & 905\end{array}$

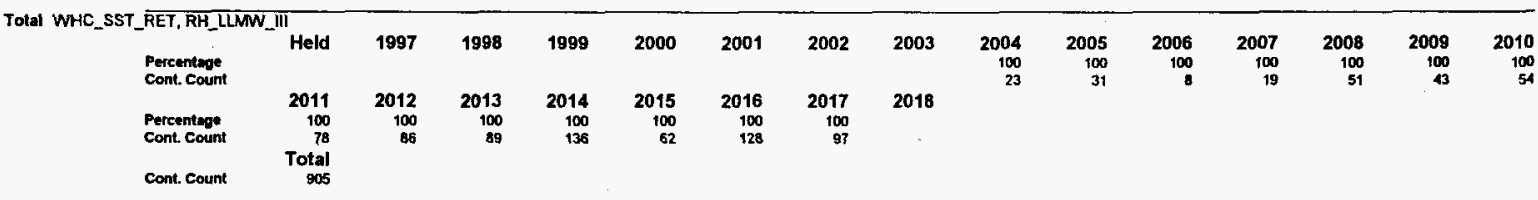


Waste Generator

Waste Class, Container, Physical Waste Form $\frac{\text { WHC_HLVP }}{\text { CH_LIW I, H-2-42701 (4x486), D INORG }}$

\begin{tabular}{|c|c|c|c|c|c|c|c|c|c|c|c|c|c|c|c|}
\hline Peccentape & Held & 1997 & 1998 & 1999 & 2000 & 2001 & $\begin{array}{r}2002 \\
100\end{array}$ & $\begin{array}{r}2003 \\
100\end{array}$ & $\begin{array}{r}2004 \\
100\end{array}$ & $\begin{array}{r}2005 \\
100\end{array}$ & $\begin{array}{r}2006 \\
100\end{array}$ & $\begin{array}{r}2007 \\
100\end{array}$ & $\begin{array}{r}2008 \\
100\end{array}$ & 2009 & 2010 \\
\hline Percentage & 2011 & 2012 & $\begin{array}{r}2013 \\
100\end{array}$ & $\begin{array}{r}2014 \\
100\end{array}$ & $\begin{array}{r}2015 \\
100\end{array}$ & $\begin{array}{r}2016 \\
100\end{array}$ & $\begin{array}{r}2017 \\
100\end{array}$ & $\begin{array}{r}2018 \\
100\end{array}$ & $\begin{array}{r}2019 \\
100\end{array}$ & $\begin{array}{r}2020 \\
100\end{array}$ & $\begin{array}{r}2021 \\
100\end{array}$ & $\begin{array}{r}2022 \\
100\end{array}$ & $\begin{array}{r}2023 \\
100\end{array}$ & $\begin{array}{r}2024 \\
100\end{array}$ & $\begin{array}{r}2025 \\
100\end{array}$ \\
\hline $\begin{array}{l}\text { Percentage } \\
\text { End of }\end{array}$ & $\begin{array}{l}2026 \\
100 \\
\text { Reached }\end{array}$ & $\begin{array}{r}2027 \\
100\end{array}$ & $\begin{array}{r}2028 \\
100\end{array}$ & & & & & & & & & & & & \\
\hline
\end{tabular}

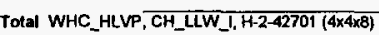

\begin{tabular}{|c|c|c|c|c|c|c|c|c|c|c|c|c|c|c|c|}
\hline Pertentage & Held & 1997 & 1098 & 1999 & 2000 & 2001 & $\begin{array}{r}2002 \\
100\end{array}$ & $\begin{array}{r}2003 \\
100\end{array}$ & $\begin{array}{r}2004 \\
100\end{array}$ & $\begin{array}{r}2005 \\
100\end{array}$ & $\begin{array}{r}2006 \\
100\end{array}$ & $\begin{array}{r}2007 \\
100\end{array}$ & $\begin{array}{r}2008 \\
100\end{array}$ & 2009 & 2010 \\
\hline Percentege & 2011 & 2012 & 2013 & 2014 & 2015 & 2016 & 2017 & $\begin{array}{r}2018 \\
100\end{array}$ & 2019 & $\begin{array}{r}2020 \\
100\end{array}$ & $\begin{array}{r}2021 \\
100\end{array}$ & $\begin{array}{r}2022 \\
100\end{array}$ & $\begin{array}{r}2023 \\
t 00\end{array}$ & $\begin{array}{r}2024 \\
100\end{array}$ & $\begin{array}{r}2025 \\
100\end{array}$ \\
\hline Percentuge & $\begin{array}{r}2026 \\
100\end{array}$ & $\begin{array}{r}2027 \\
100\end{array}$ & $\begin{array}{r}2028 \\
100\end{array}$ & & & & & & & & & & & & \\
\hline
\end{tabular}

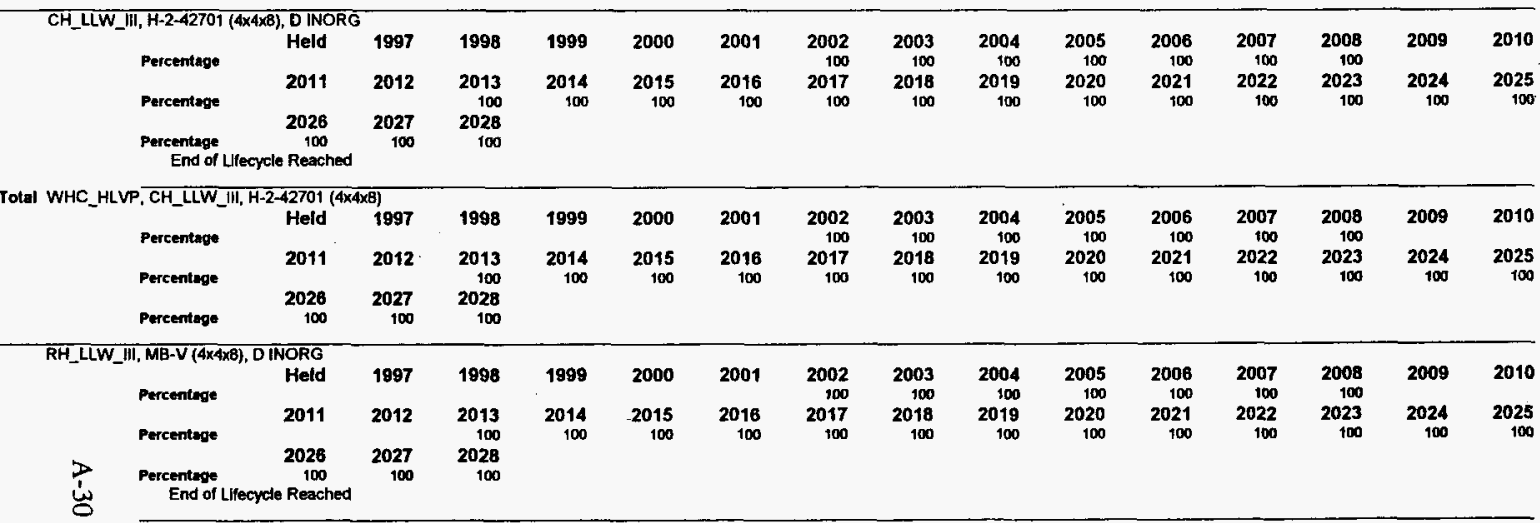




\section{Waste Generator}

Waste Class, Container, Physical Waste Form

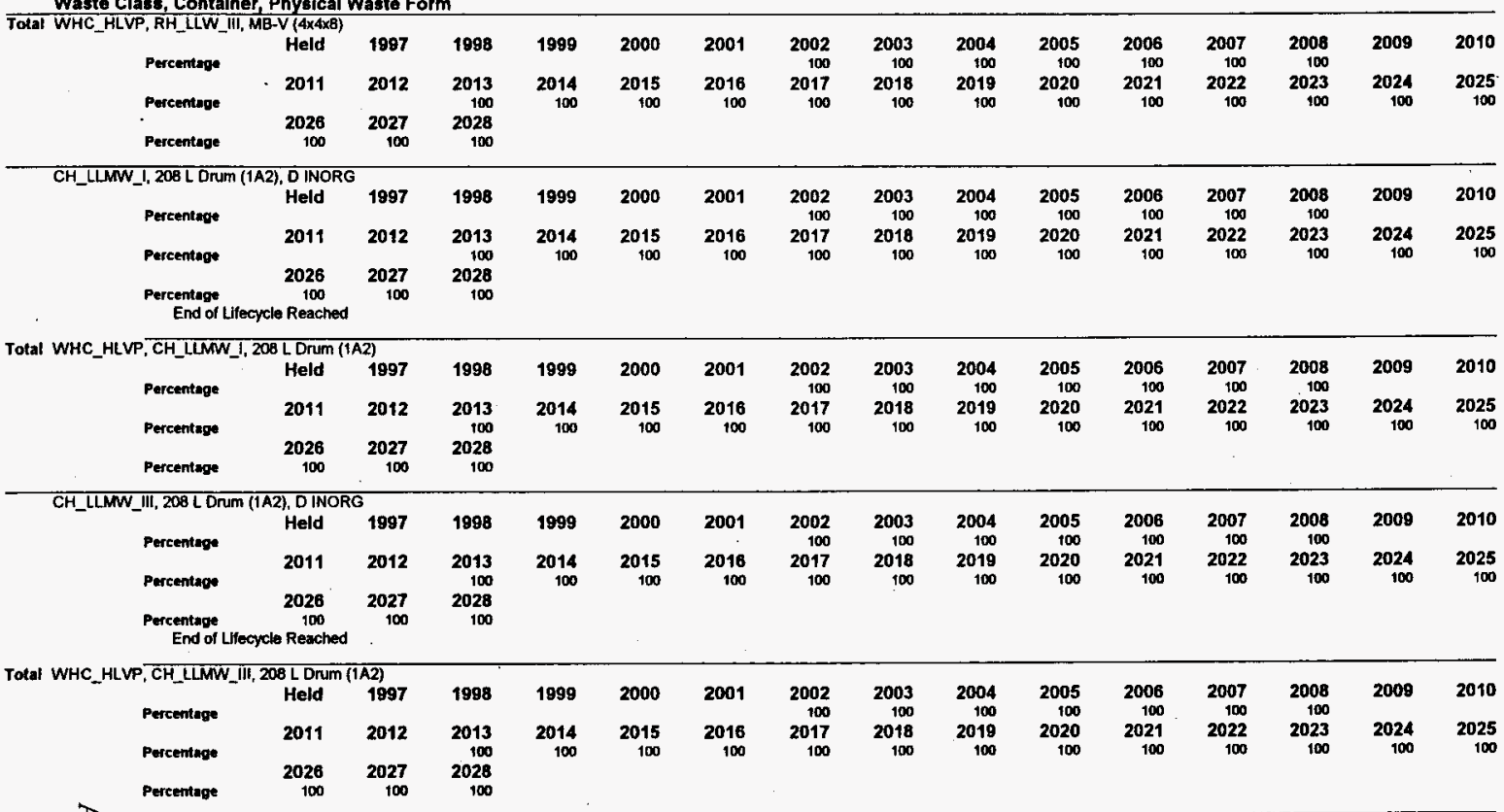

F10


Waste Generator

Waste Class, Container, Physical Waste Form

CH TRUM, 208 L D DUM (1A2), DINORG

\begin{tabular}{|c|c|c|c|c|c|c|c|c|c|c|c|c|c|c|}
\hline Percentuge & Held & 1997 & 1998 & 1999 & 2000 & 2001 & $\begin{array}{r}2002 \\
100\end{array}$ & $\begin{array}{r}2003 \\
100\end{array}$ & $\begin{array}{r}2004 \\
100\end{array}$ & $\begin{array}{r}2005 \\
.100\end{array}$ & $\begin{array}{r}2006 \\
100\end{array}$ & $\begin{array}{r}2007 \\
100\end{array}$ & $\begin{array}{r}2008 \\
100\end{array}$ & 2009 \\
\hline Percentege & 2011 & 2012 & $\begin{array}{r}2013 \\
100\end{array}$ & $\begin{array}{r}2014 \\
100\end{array}$ & $\begin{array}{r}2015 \\
100\end{array}$ & $\begin{array}{r}2016 \\
100\end{array}$ & $\begin{array}{r}2017 \\
100\end{array}$ & $\begin{array}{r}2018 \\
100\end{array}$ & $\begin{array}{r}2019 \\
100\end{array}$ & $\begin{array}{r}2020 \\
100\end{array}$ & $\begin{array}{r}2021 \\
100\end{array}$ & $\begin{array}{r}2022 \\
100\end{array}$ & $\begin{array}{r}2023 \\
100\end{array}$ & $\begin{array}{r}2024 \\
100\end{array}$ \\
\hline $\begin{array}{l}\text { Percentras } \\
\text { End of }\end{array}$ & $\begin{array}{c}2026 \\
100 \\
\text { cto Reached }\end{array}$ & $\begin{array}{r}2027 \\
100\end{array}$ & $\begin{array}{r}2028 \\
100\end{array}$ & & & & & & & & & & & \\
\hline
\end{tabular}

Total WHC_HLVP, CH_TRUM, 208 L Drum (1A2)

\begin{tabular}{|c|c|c|c|c|c|c|c|c|c|c|c|c|c|c|c|}
\hline \multicolumn{16}{|c|}{ CH_TRUM, 20B L Drum (1A2) } \\
\hline Pereenteres & Held & 1997 & 1998 & & & & $\begin{array}{r}2002 \\
100\end{array}$ & $\begin{array}{r}2003 \\
100\end{array}$ & $\begin{array}{r}2004 \\
100\end{array}$ & 2005 & $\begin{array}{r}2006 \\
100\end{array}$ & $\begin{array}{r}2007 \\
100\end{array}$ & $\begin{array}{r}2008 \\
100\end{array}$ & 2000 & 2010 \\
\hline Percentege & 2011 & 2012 & $\begin{array}{r}2013 \\
100\end{array}$ & $\begin{array}{r}2014 \\
100\end{array}$ & 2015 & $\begin{array}{r}2016 \\
100\end{array}$ & $\begin{array}{r}2017 \\
100\end{array}$ & 2018 & $\begin{array}{r}2019 \\
100\end{array}$ & $\begin{array}{r}2020 \\
100\end{array}$ & $\begin{array}{r}2021 \\
100\end{array}$ & $\begin{array}{r}2022 \\
100\end{array}$ & $\begin{array}{r}2023 \\
100\end{array}$ & $\begin{array}{r}2024 \\
100\end{array}$ & $\begin{array}{r}2025 \\
100\end{array}$ \\
\hline Peicationo & 2026 & 2027 & 2028 & & & & & & & & & 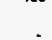 & & & \\
\hline
\end{tabular}

RH_TRUM, SW8, D INORG

$\begin{array}{llrr} & \text { Heid } & 1997 & 199 \\ \text { Percentege } & 2011 & 2012 & 2013 \\ \text { Percentage } & 2026 & 2027 & 202 \\ \text { Percentage } & 100 & 100 & 100 \\ \text { End of Ltfecycle Reached } & & \end{array}$

$\begin{array}{lllllllllllllll}1998 & 1999 & 2000 & 2004 & 2002 & 2003 & 2004 & 2005 & 2006 & 2007 & 2008 & 2009 & 2010\end{array}$

$\begin{array}{rrrrr}2013 & 2014 & 2015 & 2016 & 2017 \\ 100 & 100 & 100 & 100 & 100\end{array}$

RH_TRUM, SWB

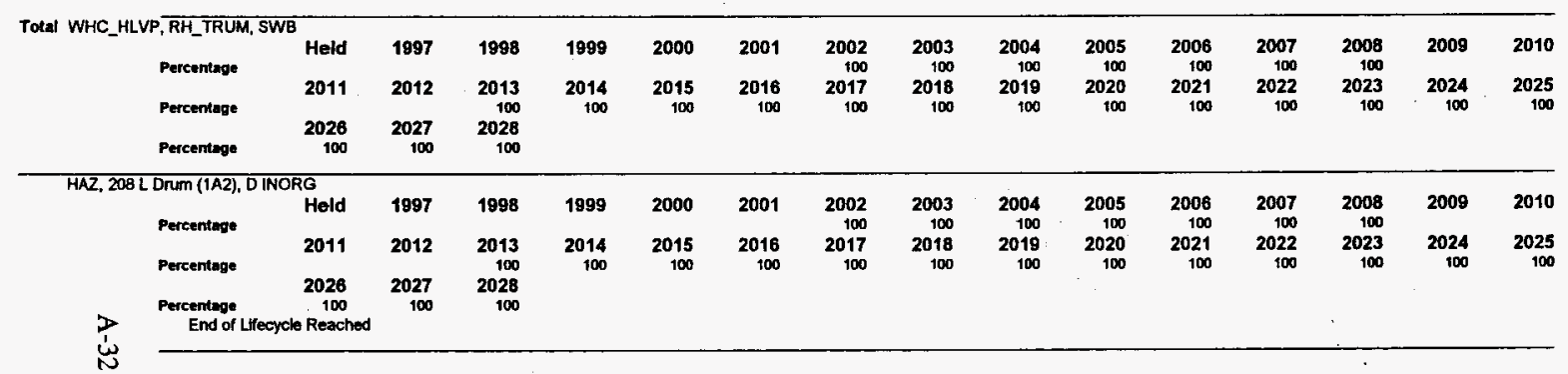


WHC-SD-WM-TI-777

Revision 0

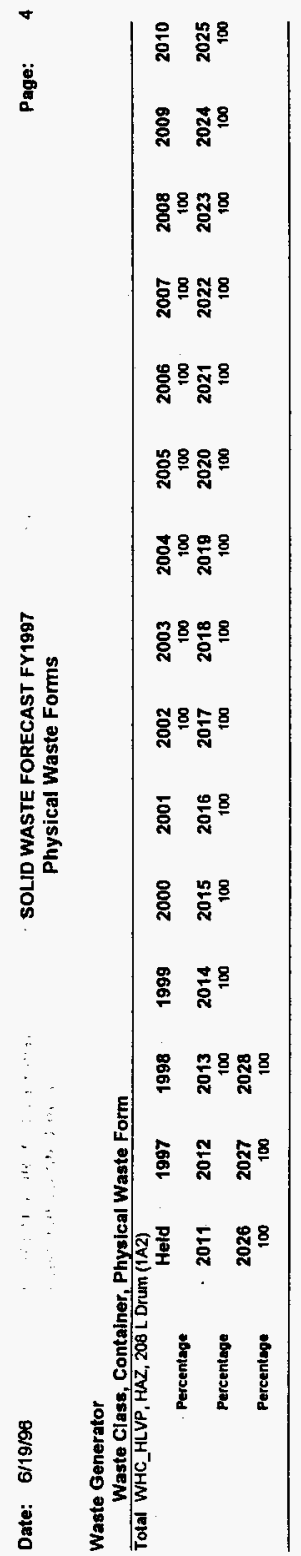

A-33 
Waste Generator

Waste Class, Container, Physical Waste Form

WHC_LLVP 2021

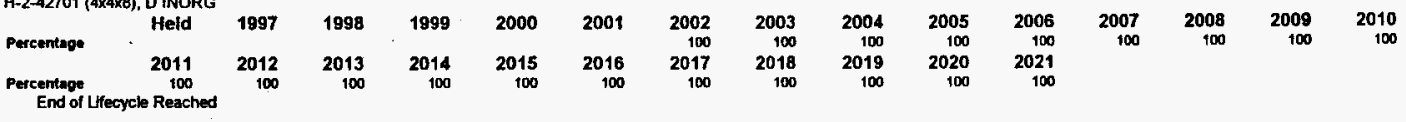

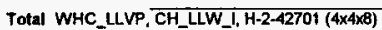

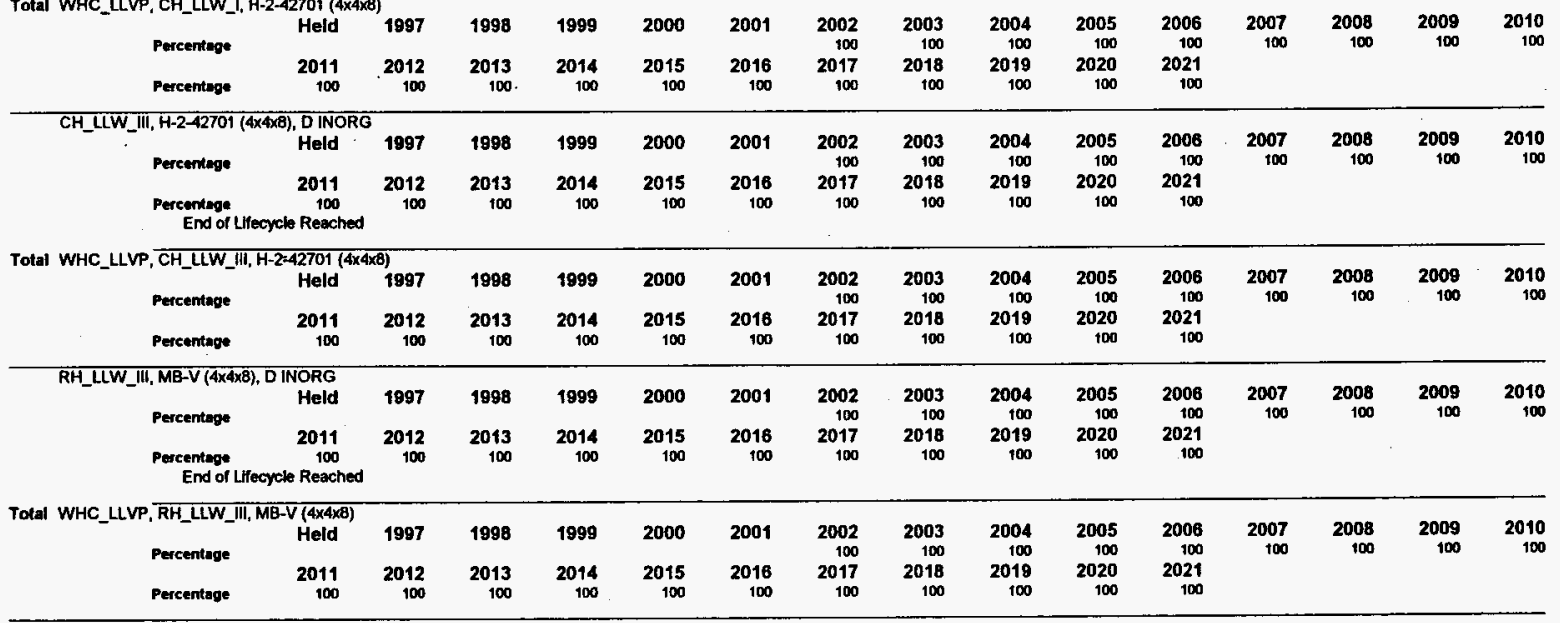

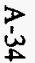


Waste Generator

Waste Class, Container, Physical Waste Form

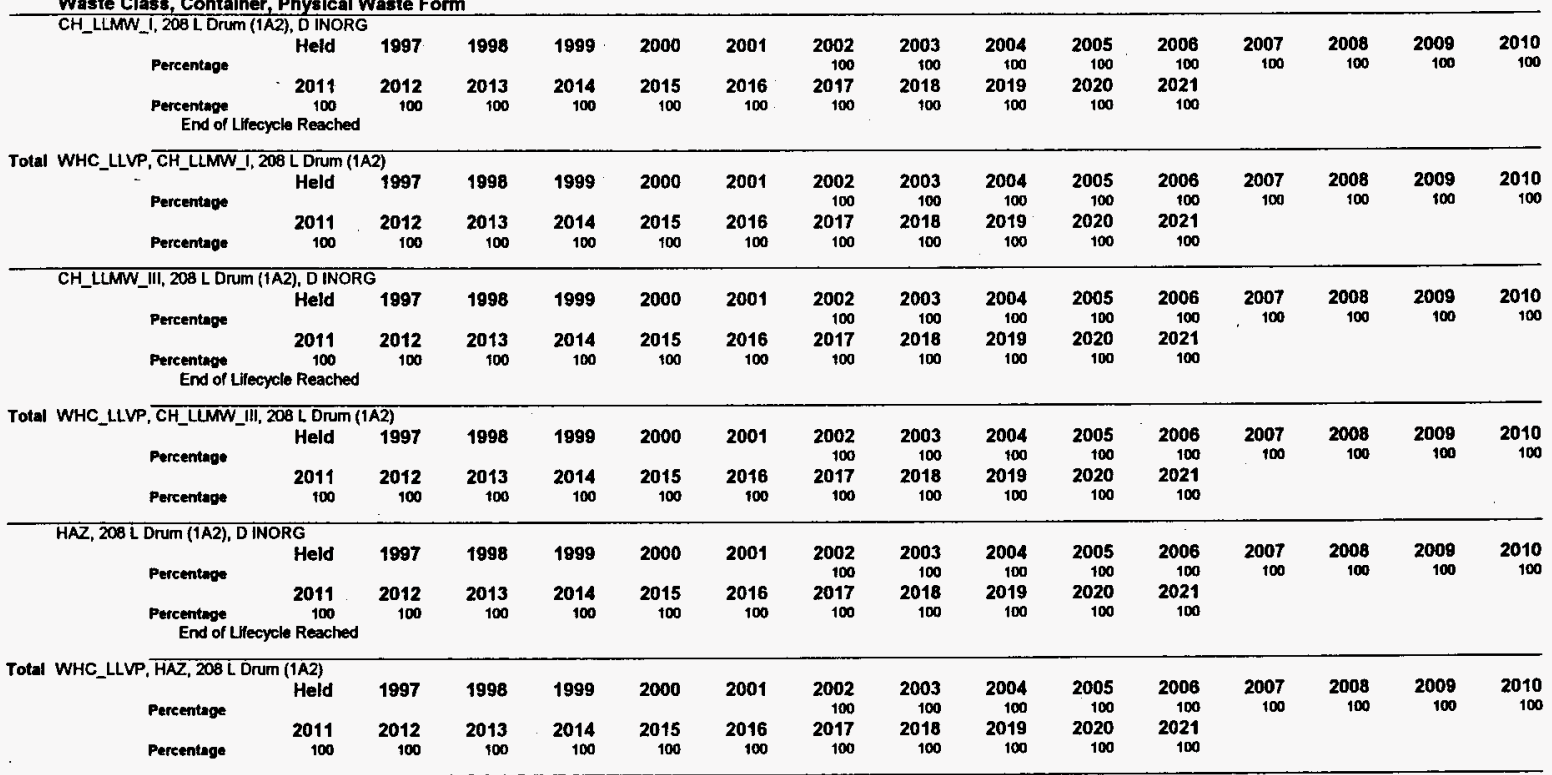

总

Physical Waste Forms 
Waste Generator

Waste Class, Container, Physical Waste Form

\begin{tabular}{c} 
WHC SST LLE \\
\hline RH_LLMW_III, LEC-10, D METAL-CONT
\end{tabular}

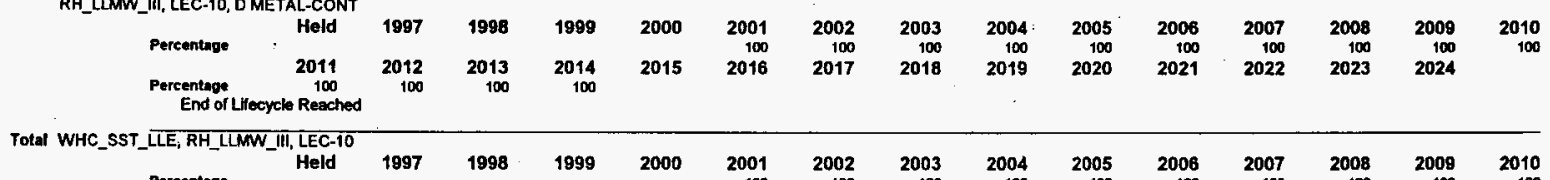

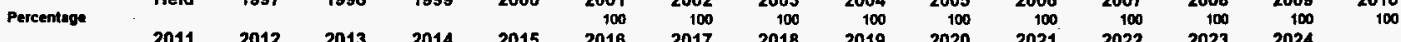

$\begin{array}{rrrrrrrrrrrrrrrr} & 2011 & 2012 & 2013 & 2014 & 2015 & 2016 & 2017 & 2018 & 2019 & 2020 & 2021 & 2022 & 2023 & 2024 & 100\end{array}$

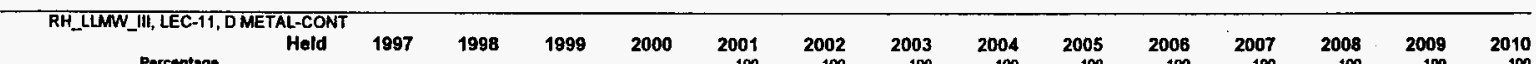

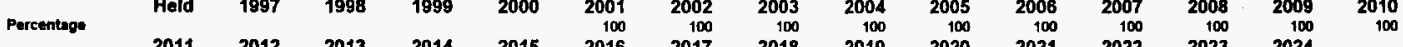

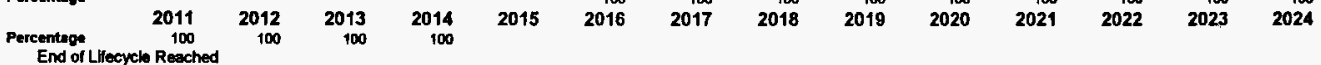

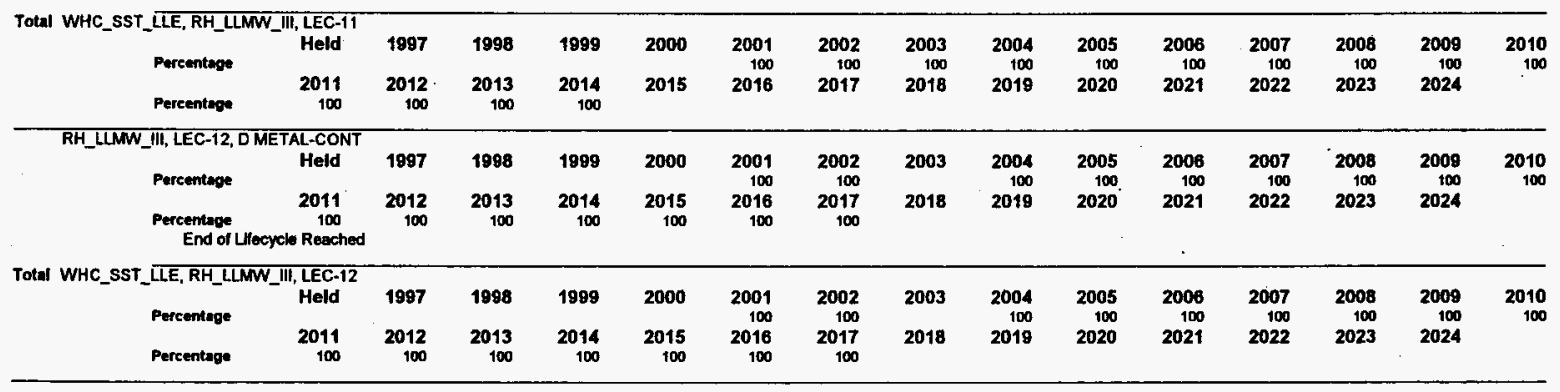

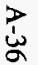


Waste Generator

Waste Class, Container, Physical Waste Form

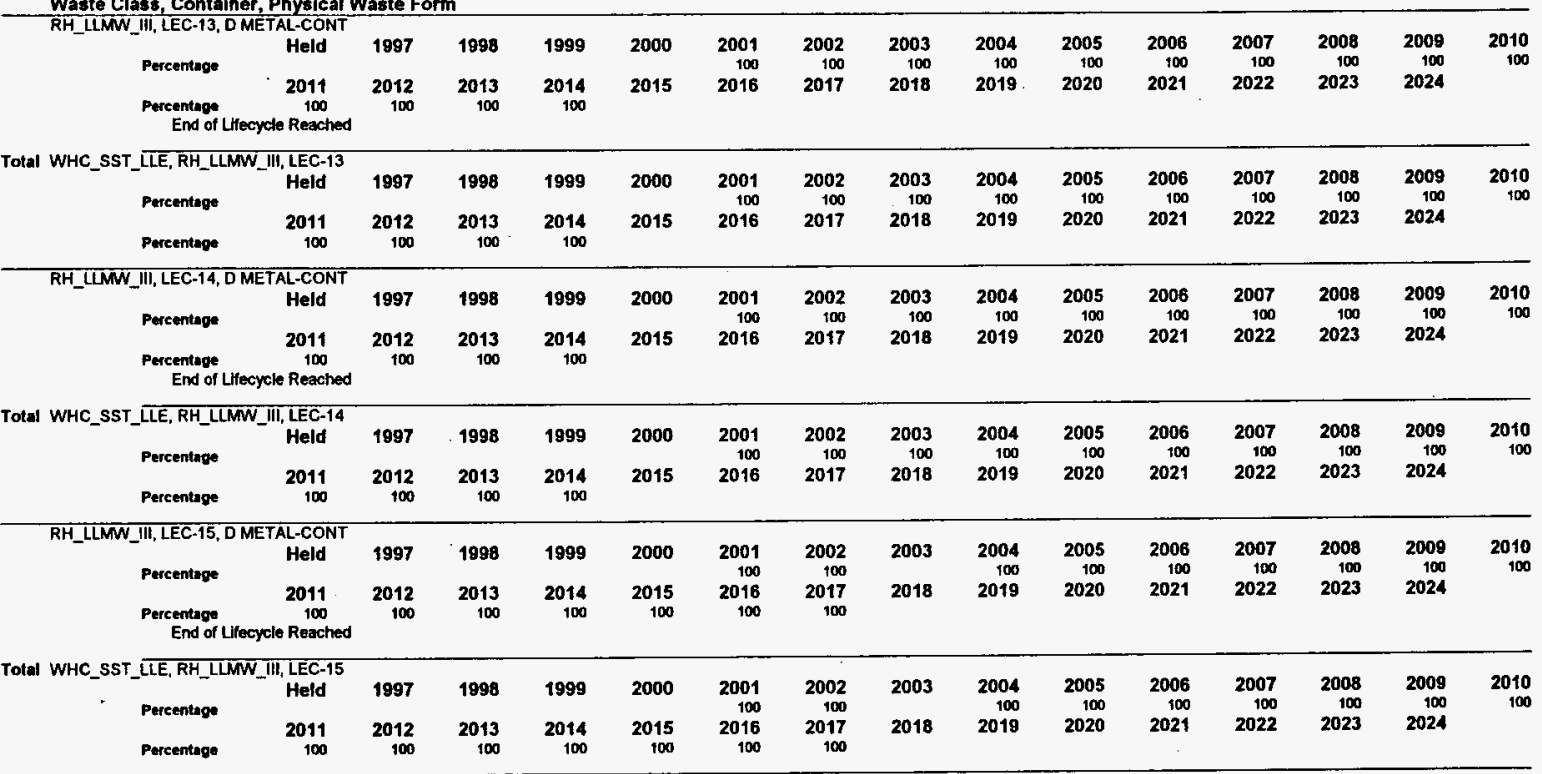

$$
\text { Physical Waste Forms }
$$


Waste Generator

Waste Class, Container, Physical Waste Form

Waste Class, Container, Physical Waste Form

\begin{tabular}{|c|c|c|c|c|c|c|c|c|c|c|c|c|c|c|c|}
\hline \multicolumn{16}{|c|}{ III, LEC-16, D METAL-CONT } \\
\hline Percentage & Held & 1997 & 1998 & 1999 & 2000 & $\begin{array}{r}2001 \\
100\end{array}$ & $\begin{array}{r}2002 \\
100\end{array}$ & 2003 & 2004 & 2005 & 2006 & 2007 & 2008 & 2009 & 2010 \\
\hline $\begin{array}{l}\text { Percentag* } \\
\text { End of } L\end{array}$ & $\begin{array}{l}2011 \\
\text { Reached }\end{array}$ & 2012 & 2013 & 2014 & 2015 & 2016 & 2017 & 2018 & 2019 & 2020 & 2021 & 2022 & 2023 & 2024 & \\
\hline
\end{tabular}

End of Lifecycle Reached
Pentegs

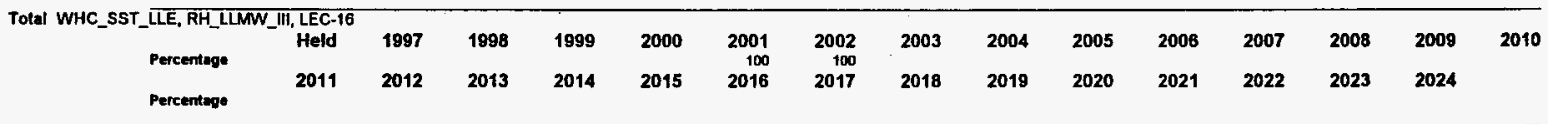

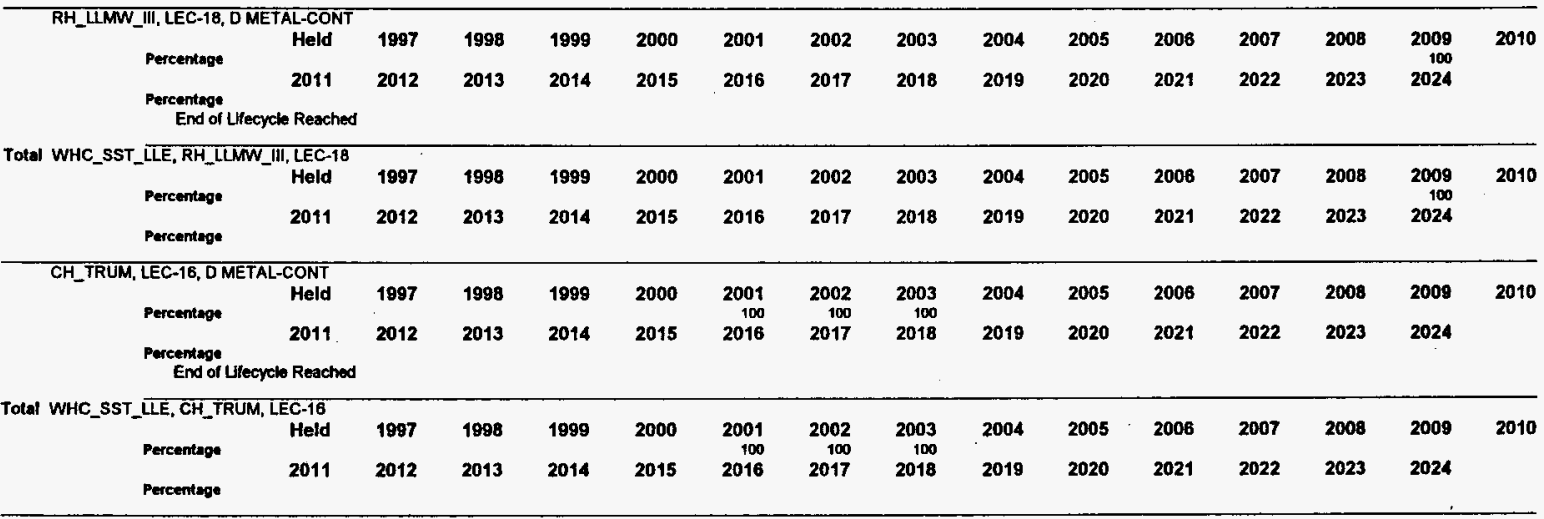


Waste Generator

Waste Class, Container, Physical Waste Form

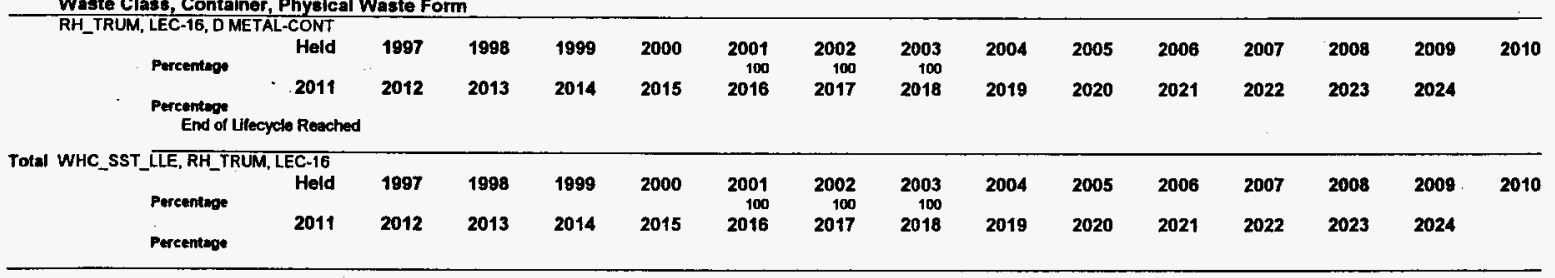


Waste Generator

Waste Class, Container, Physical Waste Form

WHC SST RET End OA Lifecycle: 2018

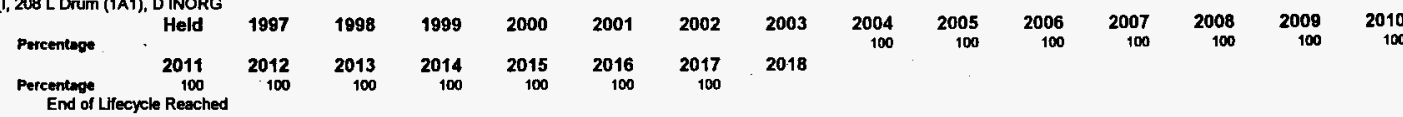

Total WHC_SST_RET, CH_LLW_I, 200 L Drum (1A1)

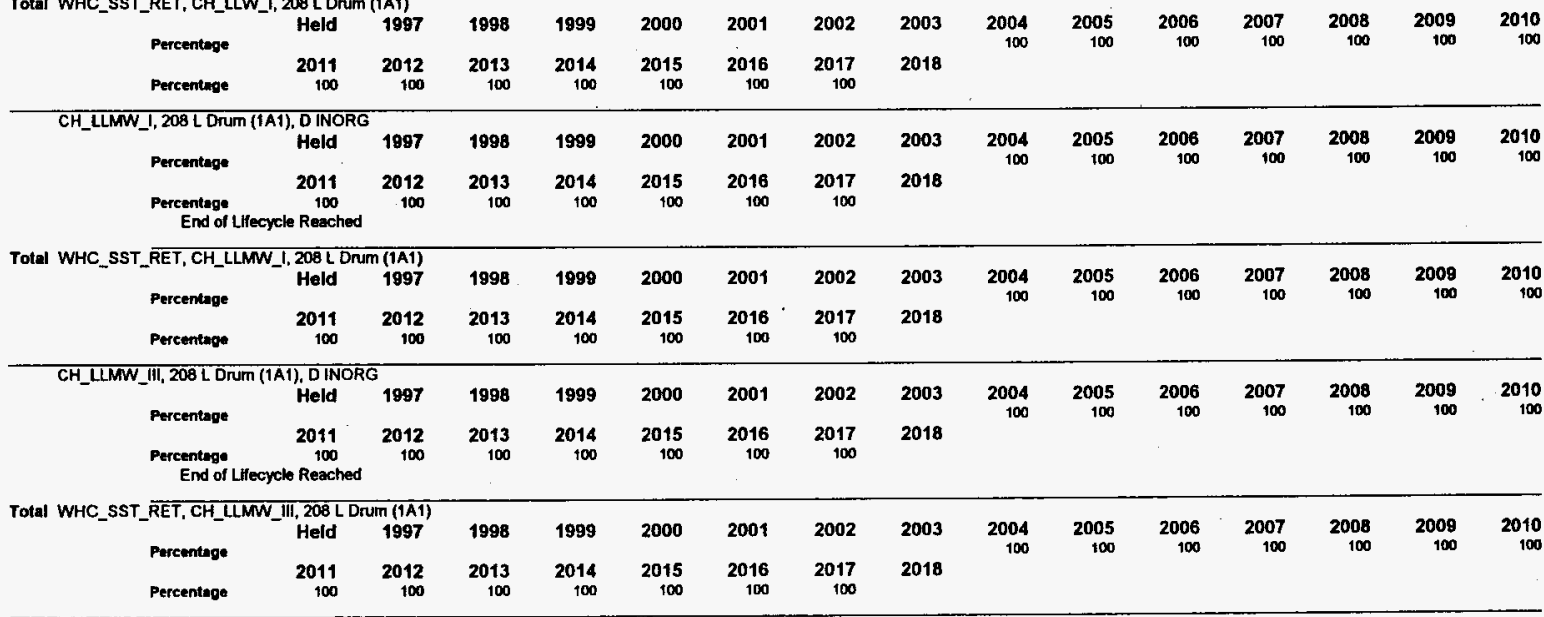

$\frac{D}{8}$ 
Waste Generator

Waste Class, Container, Physical Waste Form

Wh_LLMW_I, 208 L Drum (1A1), D INORG

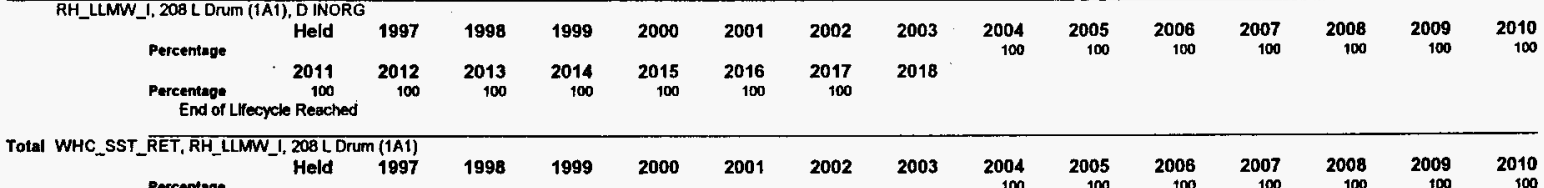

$\begin{array}{lllllllll} & 2011 & 2012 & 2013 & 2014 & 2015 & 2016 & 2017 & 2018\end{array}$

RH_LLMW_III, 208 L Drum (lead-lined), D INORG

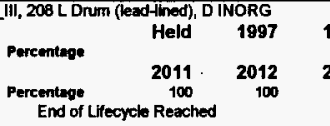

1008

100

\begin{tabular}{|c|c|c|c|c|c|c|c|c|c|c|c|c|c|c|c|}
\hline \multicolumn{16}{|l|}{ ET,RH_LL } \\
\hline ercentuge & Held & 1997 & 1998 & 1999 & 2000 & 2001 & 2002 & & $\begin{array}{r}2004 \\
100\end{array}$ & $\begin{array}{r}2005 \\
100\end{array}$ & $\begin{array}{r}2006 \\
100\end{array}$ & $\begin{array}{r}2007 \\
100\end{array}$ & $\begin{array}{r}2008 \\
100\end{array}$ & $\begin{array}{r}2009 \\
100\end{array}$ & $\begin{array}{r}2010 \\
100\end{array}$ \\
\hline ercentrge & $\begin{array}{r}2011 \\
100\end{array}$ & $\begin{array}{r}2012 \\
100\end{array}$ & $\begin{array}{r}2013 \\
100\end{array}$ & $\begin{array}{r}2014 \\
100\end{array}$ & $\begin{array}{r}2015 \\
100\end{array}$ & $\begin{array}{r}2016 \\
100\end{array}$ & $\begin{array}{r}2017 \\
100\end{array}$ & 2018 & & & & & & & \\
\hline
\end{tabular}


Waste Generator

Waste Class, Container, Physical Waste Form, Hazardous Constituent WHC HEVP

Hazardous Constituents

H.VP End O Lliecycle: 2028

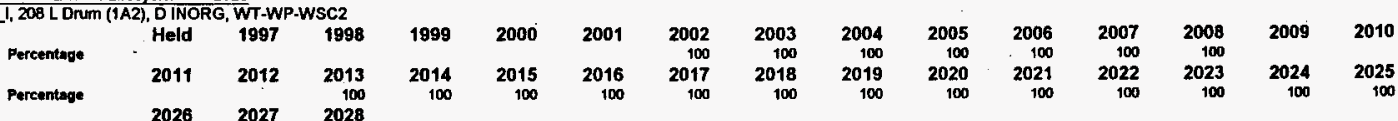

\begin{tabular}{rrrr}
2026 & 2027 & 2028 \\
\hline
\end{tabular}

End of Lifecycle Reached

Total WHC_HLVP, CH_LLMW_, 208 L Orum (1A2), DINORG

\begin{tabular}{|c|c|c|c|c|c|c|c|c|c|c|c|c|c|c|c|}
\hline \multicolumn{16}{|c|}{, CH_LLMW_1, 208 L Orum (1A2), DINORG } \\
\hline Percentage & Held & 1997 & 1998 & 1899 & 2000 & 2001 & $\begin{array}{r}2002 \\
100\end{array}$ & $\begin{array}{r}2003 \\
100\end{array}$ & $\begin{array}{r}2004 \\
100\end{array}$ & $\begin{array}{r}2005 \\
100\end{array}$ & $\begin{array}{r}2006 \\
100\end{array}$ & $\begin{array}{r}2007 \\
100\end{array}$ & $\begin{array}{r}2008 \\
100\end{array}$ & 2009 & 2010 \\
\hline Percentage & 2011 & 2012 & $\begin{array}{r}2013 \\
100\end{array}$ & $\begin{array}{r}2014 \\
100\end{array}$ & $\begin{array}{r}2015 \\
100\end{array}$ & $\begin{array}{r}2016 \\
100\end{array}$ & $\begin{array}{r}2017 \\
100\end{array}$ & $\begin{array}{r}2018 \\
100\end{array}$ & $\begin{array}{r}2019 \\
100\end{array}$ & $\begin{array}{r}2020 \\
100\end{array}$ & $\begin{array}{r}2021 \\
100\end{array}$ & $\begin{array}{r}2022 \\
100\end{array}$ & $\begin{array}{r}2023 \\
100\end{array}$ & $\begin{array}{r}2024 \\
100\end{array}$ & $\begin{array}{r}2025 \\
100\end{array}$ \\
\hline Percentege & $\begin{array}{r}2026 \\
100\end{array}$ & $\begin{array}{r}2027 \\
100\end{array}$ & $\begin{array}{r}2028 \\
100\end{array}$ & & & & & & & & & & & & \\
\hline
\end{tabular}

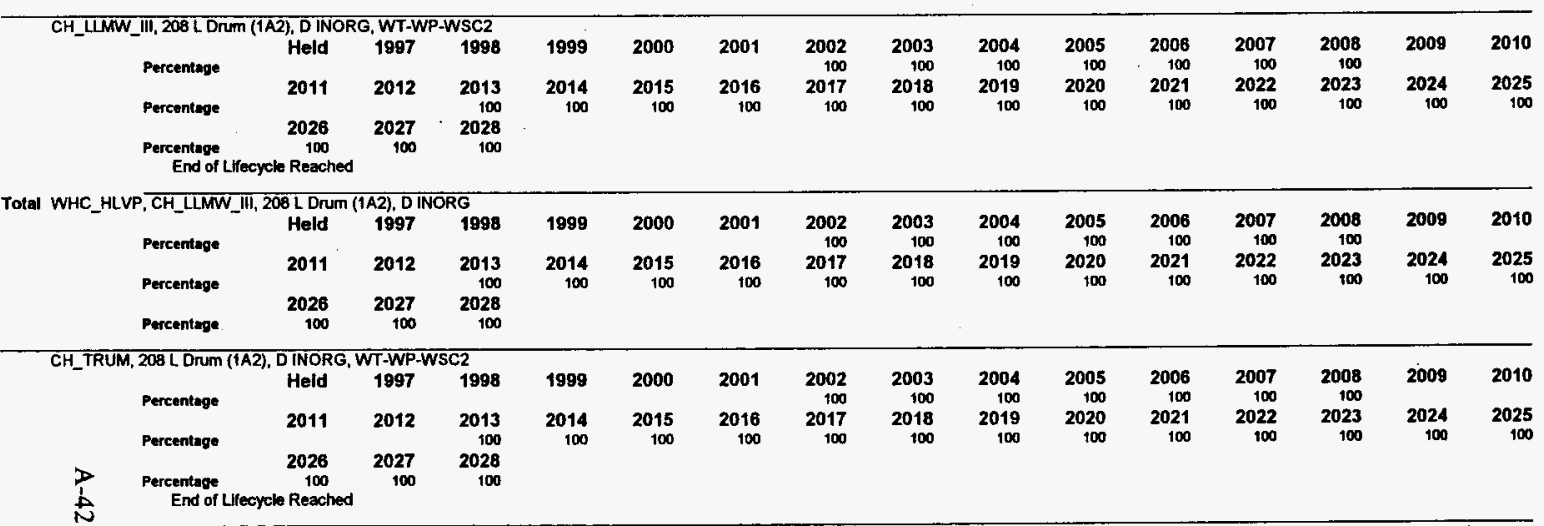


Waste Generator

Waste Class, Container, Physical Waste Form, Hazardous Constituent

Total WHC HLVP, CH TRUM, 200 L Drum (1A2) DINORG, Hazs

\begin{tabular}{|c|c|c|c|c|c|c|c|c|c|c|c|c|c|c|c|}
\hline P. CH_TRUN & Held & 1997 & 1998 & 1999 & 2000 & 2001 & $\begin{array}{r}2002 \\
100\end{array}$ & $\begin{array}{r}2003 \\
100\end{array}$ & $\begin{array}{r}2004 \\
100\end{array}$ & $\begin{array}{r}2005 \\
100\end{array}$ & $\begin{array}{r}2006 \\
100\end{array}$ & $\begin{array}{r}2007 \\
\text { t00 }\end{array}$ & $\begin{array}{r}2008 \\
100\end{array}$ & 2009 & 2010 \\
\hline Pereentage & 2011 & 2012 & $\begin{array}{r}2013 \\
100\end{array}$ & $\begin{array}{r}2014 \\
100\end{array}$ & $\begin{array}{r}2015 \\
100\end{array}$ & $\begin{array}{r}2016 \\
100\end{array}$ & $\begin{array}{r}2017 \\
100\end{array}$ & $\begin{array}{r}2018 \\
100\end{array}$ & $\begin{array}{r}2019 \\
100\end{array}$ & $\begin{array}{r}2020 \\
100\end{array}$ & $\begin{array}{r}2021 \\
100\end{array}$ & $\begin{array}{r}2022 \\
100\end{array}$ & $\begin{array}{r}2023 \\
100\end{array}$ & $\begin{array}{r}2024 \\
100\end{array}$ & $\begin{array}{r}2025 \\
100\end{array}$ \\
\hline & 2026 & 2027 & 2028 & & & & & & & & & & & & \\
\hline Percentrge & 100 & 100 & 100 & & & & & & & & & & & & \\
\hline
\end{tabular}

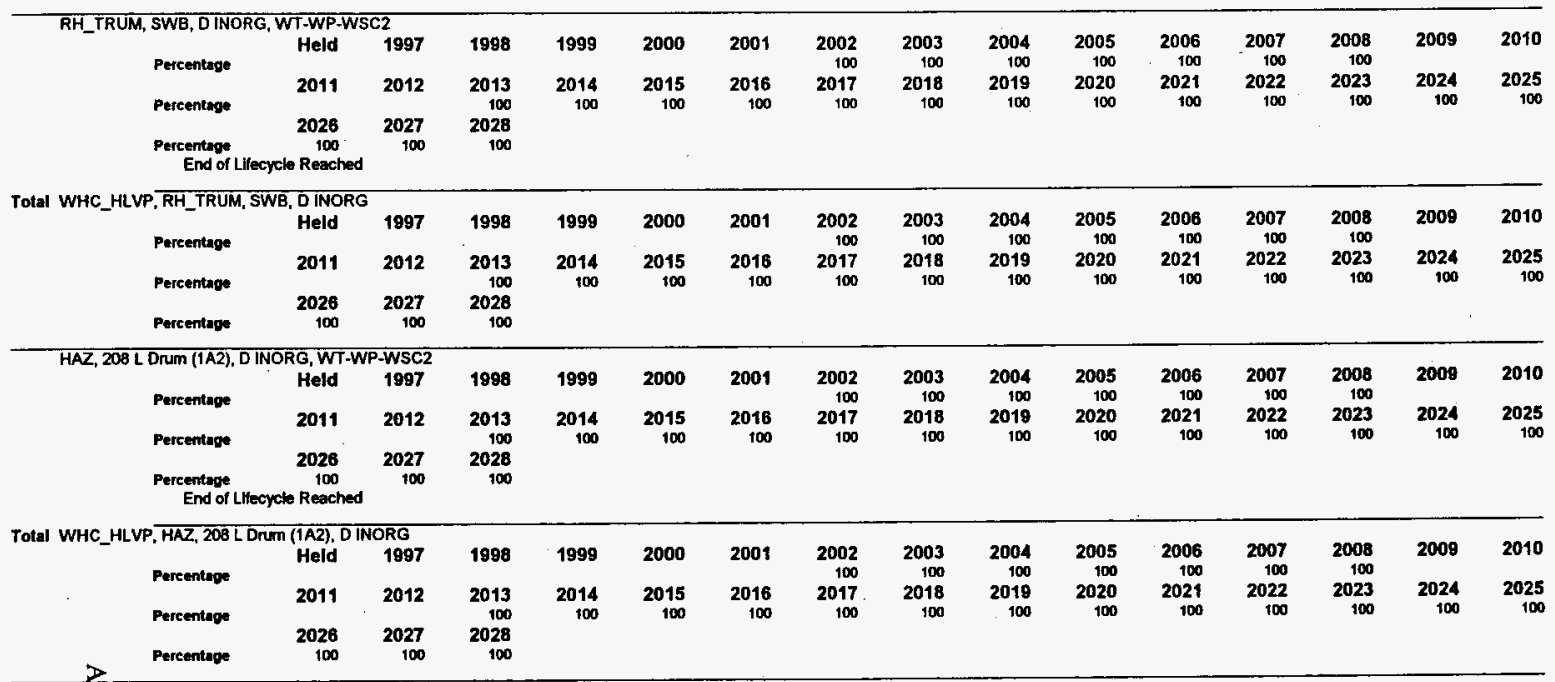

$\omega$ 
Waste Generator

Waste Class, Container, Physical Waste Form, Hazardous Constituent WHC_LLVP $\frac{\text { End Or Lifecrcle: }}{\text { CH_LLMW_I, } 200 \text { L L DUM (1A2), D INORG, WT-WP-WSC2 }}$

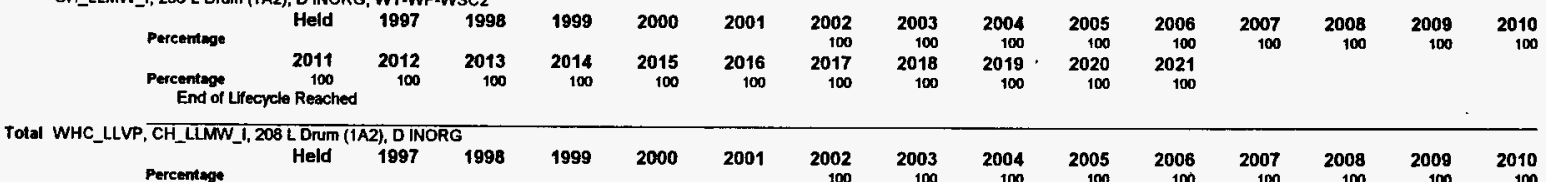

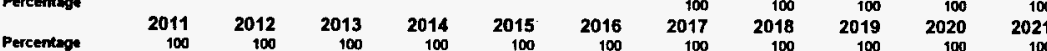

\begin{tabular}{|c|c|c|c|c|c|c|c|c|c|c|c|c|c|c|c|}
\hline \multicolumn{16}{|c|}{ CH_LLMW_III, 208 L Drum (1A2), DINORG, WT-WP-WSC } \\
\hline Percentage & Held & 1997 & 1998 & 1999 & 2000 & 2001 & $\begin{array}{r}2002 \\
100\end{array}$ & $\begin{array}{r}2003 \\
100\end{array}$ & $\begin{array}{r}2004 \\
100\end{array}$ & $\begin{array}{r}2005 \\
100\end{array}$ & $\begin{array}{r}2006 \\
100\end{array}$ & $\begin{array}{r}2007 \\
100\end{array}$ & $\begin{array}{r}2008 \\
100\end{array}$ & $\begin{array}{r}2009 \\
100\end{array}$ & $\begin{array}{r}2010 \\
100\end{array}$ \\
\hline Percentuge & $\begin{array}{r}2011 \\
100\end{array}$ & $\begin{array}{r}2012 \\
100\end{array}$ & $\begin{array}{r}2013 \\
100\end{array}$ & $\begin{array}{r}2014 \\
100\end{array}$ & $\begin{array}{r}2015 \\
100\end{array}$ & $\begin{array}{r}2016 \\
\text { t00 }\end{array}$ & $\begin{array}{r}2017 \\
100\end{array}$ & $\begin{array}{r}2018 \\
100\end{array}$ & $\begin{array}{r}2019 \\
100\end{array}$ & $\begin{array}{r}2020 \\
100\end{array}$ & $\begin{array}{r}2021 \\
700\end{array}$ & & & & \\
\hline
\end{tabular}

$$
\text { End of Lifecycle Reached }
$$

Total WHC_LLVP, CH_LLMW_ili, 208 L Drum (1A2), DINORG

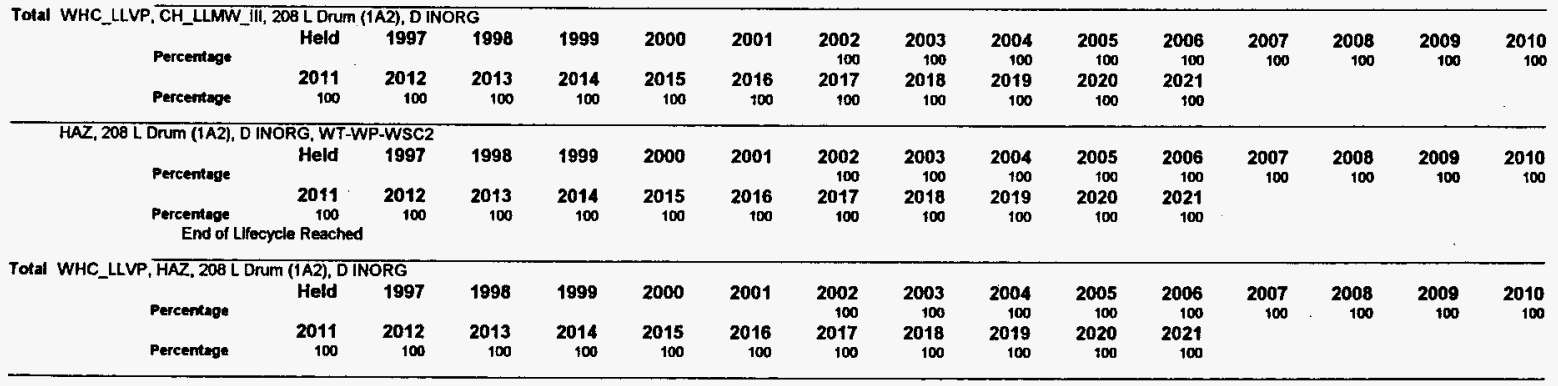

$\stackrel{p}{+}$ 


\section{Waste Generator}

Waste Class, Container, Physical Waste Form, Hazardous Constituent

WHC SST LLE End Of Lifecycle: 2024

Hazardous Constituents

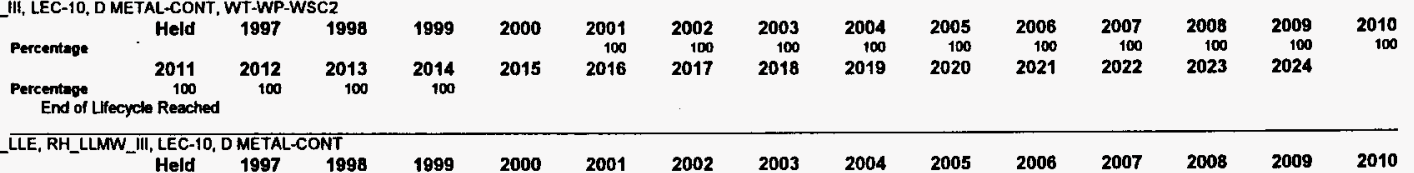

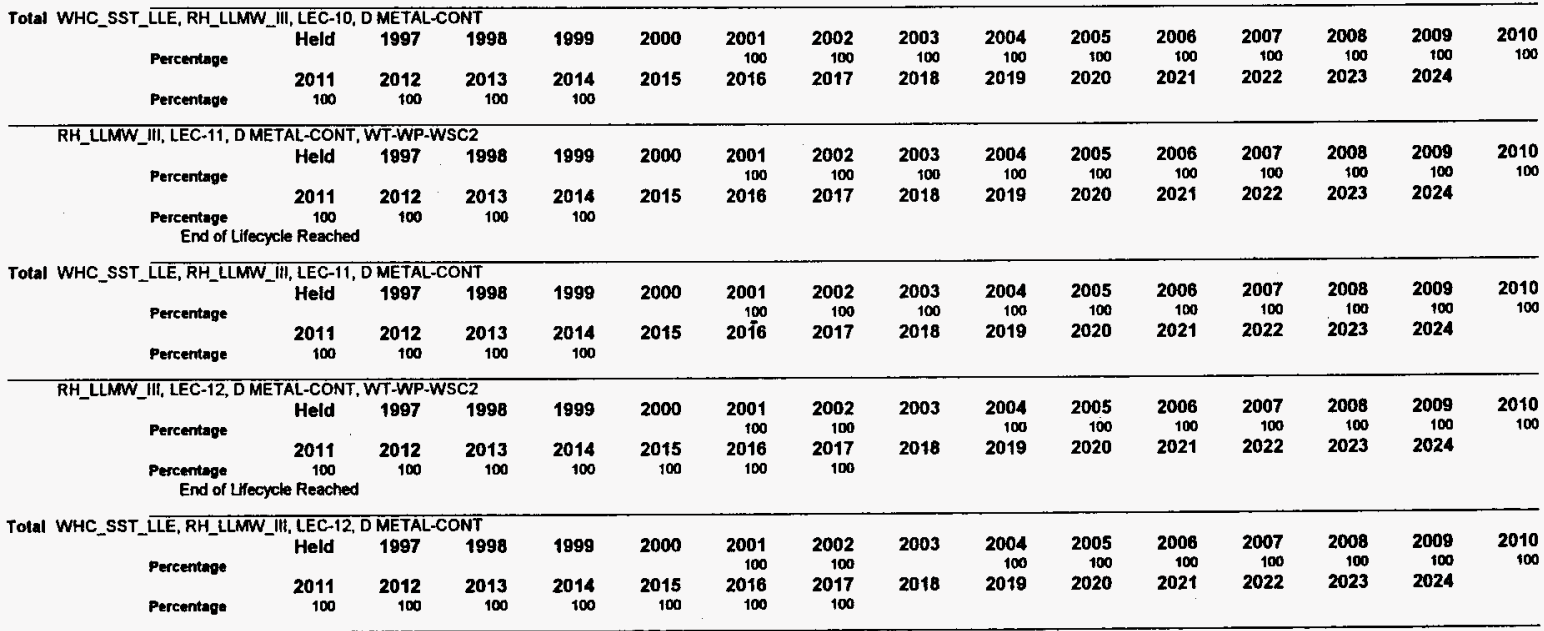

点 
Waste Generator

Waste Class, Container, Physical Waste Form, Hazardous Constituent

RH LIMW III, LEC-13, D METAL-CONT, WT-WP-WSC2

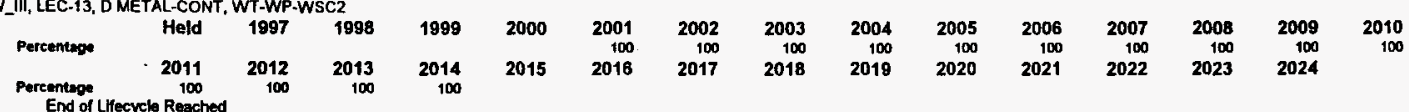

End of Lifecycle Reached

Total WHC_SST_LEE, RH_LLMW_III, LEC-13, DMETAL-CONT

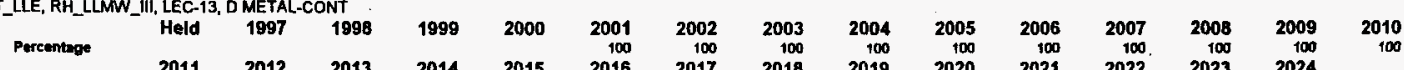

$\begin{array}{rrrr} & 2011 & 2012 & 2013 \\ \text { Percentage } & 100 & 100 & 100\end{array}$

RH_LLWW_III, LEC-14, DMETAL-CONT, WT-WP.WSC2

\begin{tabular}{|c|c|c|c|c|c|c|c|c|c|c|c|c|c|c|c|}
\hline \multicolumn{16}{|c|}{ III, LEC-14, D METAL-CONT, WT-WP.WSC2 } \\
\hline Percentuge & Held & 1997 & 1998 & 1999 & 2000 & $\begin{array}{r}2001 \\
100\end{array}$ & $\begin{array}{r}2002 \\
100\end{array}$ & $\begin{array}{r}2003 \\
100\end{array}$ & $\begin{array}{r}2004 \\
100\end{array}$ & $\begin{array}{r}2005 \\
100\end{array}$ & $\begin{array}{r}2006 \\
100\end{array}$ & $\begin{array}{r}2007 \\
100\end{array}$ & $\begin{array}{r}2008 \\
100\end{array}$ & $\begin{array}{r}2009 \\
100\end{array}$ & $\begin{array}{r}2010 \\
100\end{array}$ \\
\hline $\begin{array}{l}\text { Percentuge } \\
\text { End of } L\end{array}$ & $\begin{array}{c}2011 \\
100 \\
\text { te Reached }\end{array}$ & $\begin{array}{r}2012 \\
100\end{array}$ & $\begin{array}{r}2013 \\
100\end{array}$ & $\begin{array}{r}2014 \\
100\end{array}$ & 2015 & 2016 & 2017 & 2018 & 2019 & 2020 & 2021 & 2022 & 2023 & 2024 & \\
\hline
\end{tabular}

Total WHC_SST_LLE, RH_LLMW_III, LEC-14, DMETALICONT

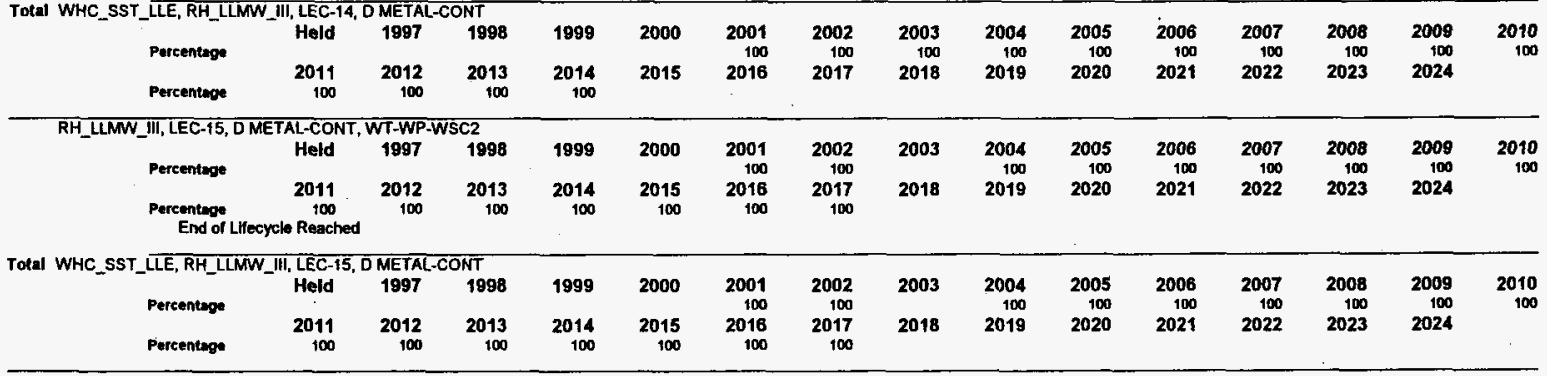

$\frac{1}{b}$ 


\section{Naste Generator}

Waste Class, Contalner, Physical Waste Form, Hazardous Constltuent

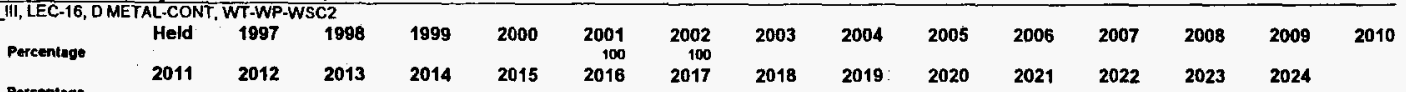

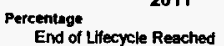

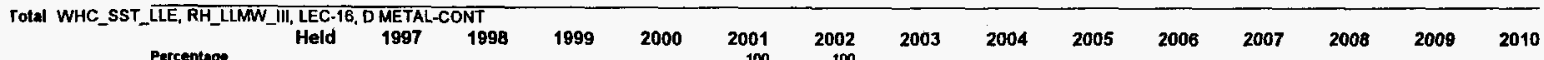

Percentage

$2011 \quad 2012 \quad 2013 \quad 2014 \quad 2015 \quad 2016$

Percentage

RH_LLMW_III, LEC-18, DMETAL-CONT, WT-WP-WSC2

\begin{tabular}{|c|c|c|c|c|c|c|c|c|c|c|c|c|c|c|}
\hline ercente & $\begin{array}{l}\text { Held } \\
2011\end{array}$ & $\begin{array}{l}1997 \\
2012\end{array}$ & $\begin{array}{l}1998 \\
2013\end{array}$ & $\begin{array}{r}1999 \\
2014\end{array}$ & $\begin{array}{l}2000 \\
2015\end{array}$ & $\begin{array}{l}2001 \\
2016\end{array}$ & $\begin{array}{l}2002 \\
2017\end{array}$ & $\begin{array}{l}2003 \\
2018\end{array}$ & $\begin{array}{l}2004 \\
2019\end{array}$ & $\begin{array}{l}2005 \\
2020\end{array}$ & $\begin{array}{l}2006 \\
2021\end{array}$ & $\begin{array}{l}2007 \\
2022\end{array}$ & $\begin{array}{l}2008 \\
2023\end{array}$ & $\begin{array}{r}2009 \\
100 \\
2024\end{array}$ \\
\hline
\end{tabular}

End of Lifecycle Reached

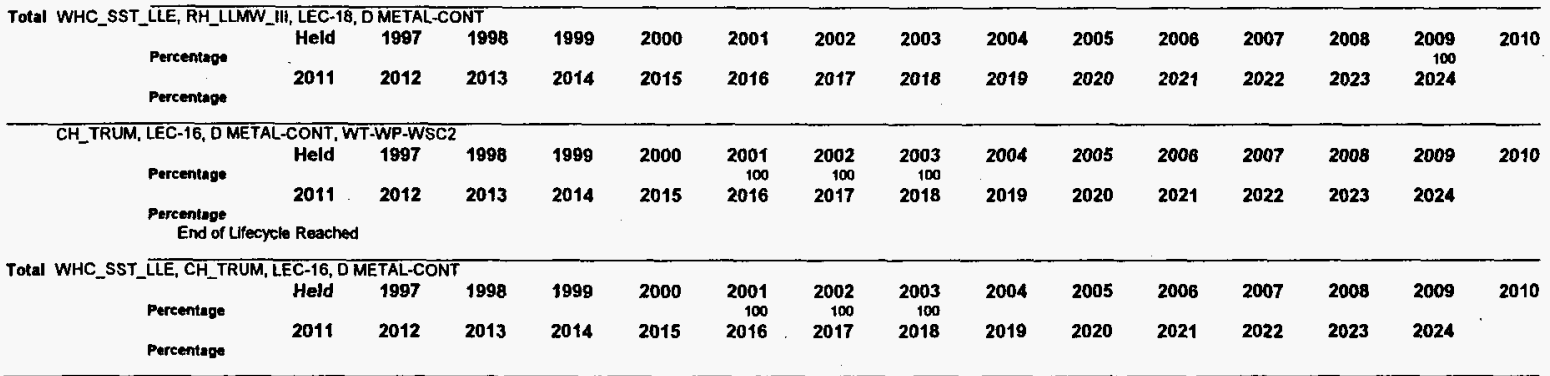

$\frac{3}{3}$ 


\section{Waste Generator}

Waste Class, Container, Physical Waste Form, Hazardous Constituent

RH_TRUM, LEC-16, D METAL-CONT, WT-WP-WSC2

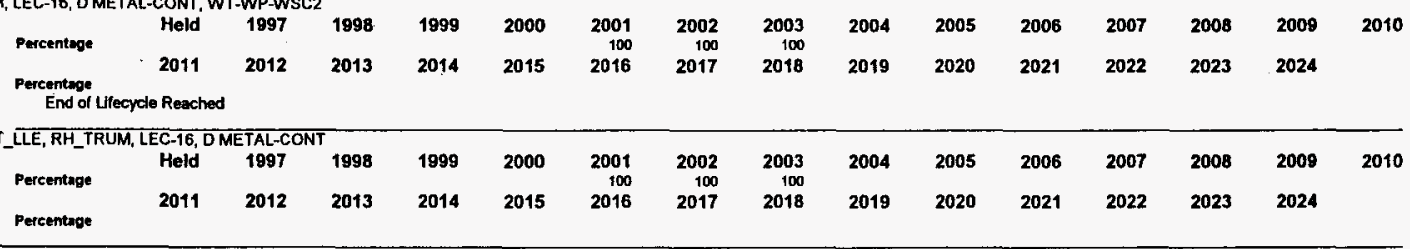


Waste Generator

Waste Class, Container, Physlcal Waste Form, Hazardous Constituent CH_LLMW_I, 208 L Drum (1A1), DINORG WT-WP-WSC2

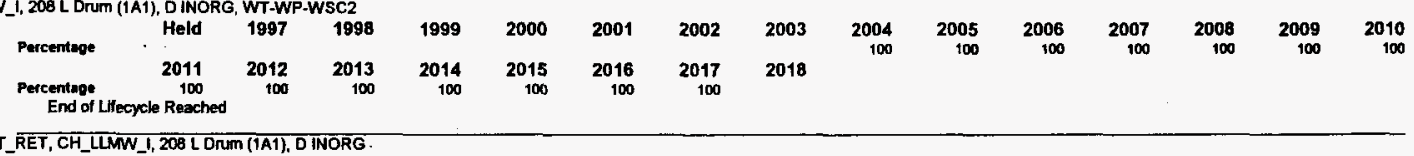

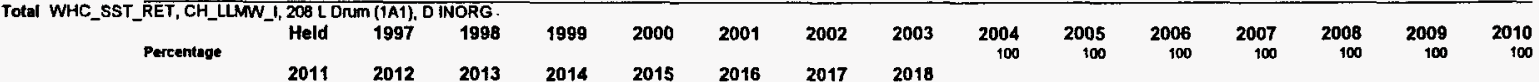

$\begin{array}{rrrrrrrrr} & 2011 & 2012 & 2013 & 2014 & 2015 & 2016 & 2017 & 2018\end{array}$

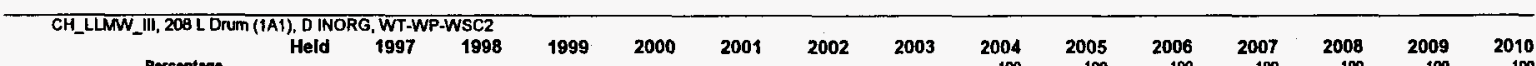

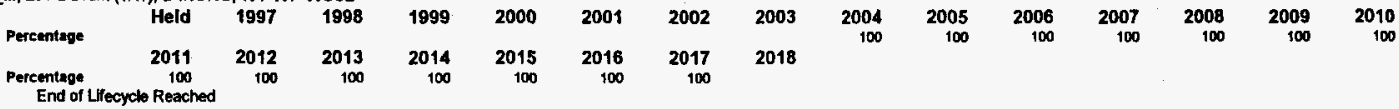
End of Lifecycle Reached

Total WHC_SST_ RET, CH_LWMWIIII, 208 LOTUm (1A1), OINORG

\begin{tabular}{|c|c|c|c|c|c|c|c|c|c|c|c|c|c|c|c|}
\hline RET,CH_LU & III, $208 \mathrm{~L}$ & $\overline{m(1 \mathrm{~A} 1)}$ & NORG & & & & & & & & & & & & \\
\hline & Held & 1997 & 1998 & 1999 & 2000 & 2001 & 2002 & & $\begin{array}{r}2004 \\
100\end{array}$ & $\begin{array}{r}2005 \\
100\end{array}$ & $\begin{array}{r}2006 \\
100\end{array}$ & $\begin{array}{r}2007 \\
100\end{array}$ & $\begin{array}{r}2008 \\
100\end{array}$ & $\begin{array}{r}2009 \\
100\end{array}$ & $\begin{array}{r}2010 \\
100\end{array}$ \\
\hline ercentuge & $\begin{array}{r}2011 \\
100\end{array}$ & $\begin{array}{r}2012 \\
100\end{array}$ & $\begin{array}{r}2013 \\
100\end{array}$ & $\begin{array}{r}2014 \\
100\end{array}$ & $\begin{array}{r}2015 \\
\text { t00 }\end{array}$ & $\begin{array}{r}2016 \\
100\end{array}$ & $\begin{array}{r}2017 \\
100\end{array}$ & 2018 & & & & & & & \\
\hline
\end{tabular}

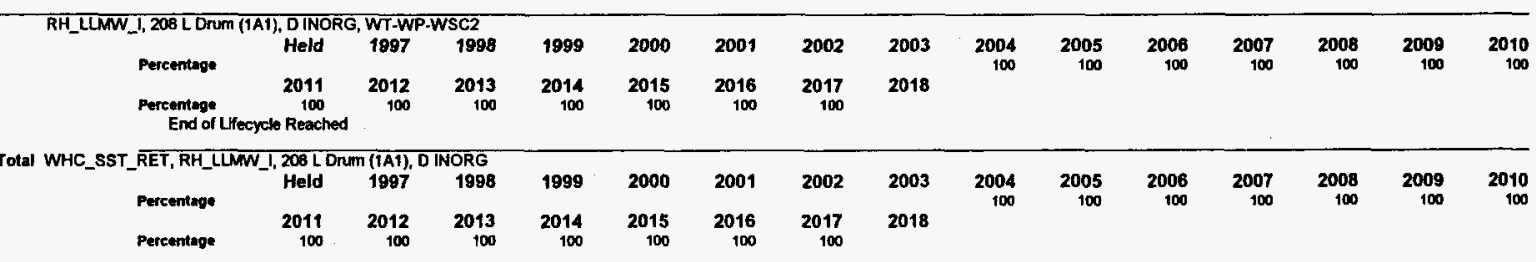

$\frac{1}{b}$

F104 Legend: $A=$ Ignituble, $B=$ Corrosive, $C=$ Reactive, $D=$ Metats w/o $\mathrm{Hg}, E=$ Metals w $\mathrm{Hg}, F=$ Organic, $G=W T, W P, W S C 2, H=P C B<50$ ppm, J $=P C B>=50$ ppm 
Waste Generator

Radionuclide Concentrations

(in curies per cubic meter)

Waste Class, Container, Physical Waste Form, Radionuclide WHC HLVP End Of Lifecycle: 2028

CH_LLW_I, H-2-42701 (4X4x8), O INORG, CESIUM137

\begin{tabular}{|c|c|c|c|c|c|c|c|c|c|c|c|c|c|c|}
\hline cmengentran & $\begin{array}{l}D \text { INOR } \\
\text { Held }\end{array}$ & $\begin{array}{l}\text { CESIUM } \\
1997\end{array}$ & 1998 & 1999 & 2000 & 2001 & 2002 & 2003 & 2004 & 2005 & 2006 & 2007 & 2008 & 2009 \\
\hline 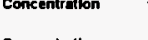 & 2011 & 2012 & 2013 & 2014 & 2015 & 2016 & $\begin{array}{r}001 \\
2017\end{array}$ & $\begin{array}{r}.001 \\
2018\end{array}$ & $\begin{array}{r}.001 \\
2019\end{array}$ & $\begin{array}{r}.001 \\
2020\end{array}$ & $\begin{array}{r}.001 \\
2021\end{array}$ & $\begin{array}{r}.001 \\
2022\end{array}$ & $\begin{array}{r}.01 \\
2023\end{array}$ & 2024 \\
\hline $\begin{array}{l}\text { Concentration } \\
\text { Concentretion }\end{array}$ & $\begin{array}{r}2026 \\
.001\end{array}$ & 2027 & $\begin{array}{r}.001 \\
2028\end{array}$ & & & & & & & & & & & \\
\hline
\end{tabular}

End of lifecycte Reached $\quad .001$

CH_LLW_I, H-2-42701 (4x4488), O INORG, STRONTIUM9O

\begin{tabular}{|c|c|c|c|c|c|c|c|c|c|c|c|c|c|c|c|}
\hline Concentration & Held & 1997 & 1998 & 1999 & 2000 & 2001 & $\begin{array}{r}2002 \\
.005\end{array}$ & $\begin{array}{r}2003 \\
.001\end{array}$ & $\begin{array}{r}2004 \\
.001\end{array}$ & $\begin{array}{r}2005 \\
.001\end{array}$ & $\begin{array}{r}2006 \\
.001\end{array}$ & $\begin{array}{r}2007 \\
.001\end{array}$ & $\begin{array}{r}2008 \\
.001\end{array}$ & 2009 & 2010 \\
\hline Concentration & 2011 & 2012 & $\begin{array}{r}2013 \\
.001\end{array}$ & $\begin{array}{r}2014 \\
.001\end{array}$ & $\begin{array}{r}2015 \\
.001\end{array}$ & $\begin{array}{r}2016 \\
.001\end{array}$ & $\begin{array}{r}2017 \\
.001\end{array}$ & $\begin{array}{r}2018 \\
.001\end{array}$ & $\begin{array}{r}2019 \\
.001\end{array}$ & $\begin{array}{r}2020 \\
.001\end{array}$ & $\begin{array}{r}2021 \\
.001\end{array}$ & $\begin{array}{r}2022 \\
.001\end{array}$ & $\begin{array}{r}2023 \\
.001\end{array}$ & $\begin{array}{r}2024 \\
.001\end{array}$ & $\begin{array}{r}2025 \\
.001\end{array}$ \\
\hline $\begin{array}{l}\text { Concentration } \\
\text {. End of Ufec }\end{array}$ & $\begin{array}{r}2026 \\
.001 \\
\text { Reached }\end{array}$ & $\begin{array}{r}2027 \\
.001\end{array}$ & $\begin{array}{r}2028 \\
.001\end{array}$ & & & & & & & & & & & & \\
\hline
\end{tabular}

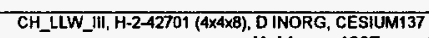

Concentration

Held

19971998

$19992000 \quad 2001$

2002

Concentration

$2011 \quad 2012 \quad 2013$
10

2007
10
2022
10

$\begin{array}{rrrr} & 2026 & 2027 & 2028 \\ \text { Concentration } & 10 & 10 & 10\end{array}$

End of Ufecycle Reached

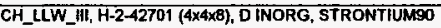

$\begin{array}{llll}\text { concentuation } & \text { Held } & 1997 & 1998 \\ \text { concentration } & 2011 & 2012 & 2013 \\ & 2026 & 2027 & 2028 \\ & 10 & 10 & \end{array}$
10
Concentration
End of Llfecycle Reached

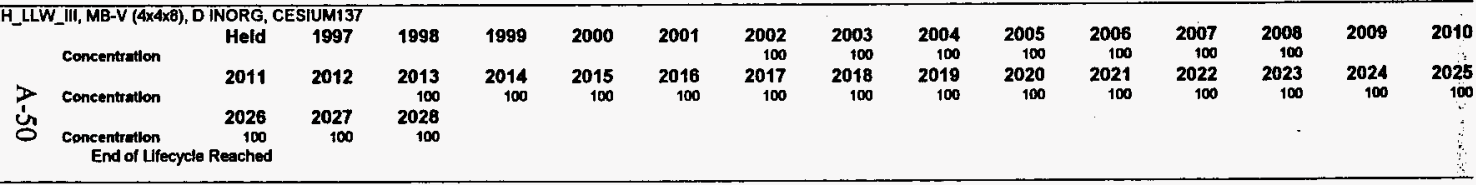


Waste Class, Contalner, Physical Waste Form, Radionuclide

RH_LLW_ili, MB-V $(4 \times 4 \times 8)$, D INORG, STRONTIUMMO

$\begin{array}{lcccc}\text { Concentration } & \text { Held } & 1997 & 1998 & 1999 \\ & 2011 & 2012 & 2013 & 2014 \\ \text { Concentration } & & & 100 & \\ & 2026 & 2027 & 2028 & \\ \begin{array}{c}\text { Concentration } \\ \text { End of Llfecycle Reached }\end{array} & 100 & 100 & \\ & & & & \end{array}$

$1999 \quad 2000 \quad 2001 \quad 2002 \quad 200$

2003

$\begin{array}{rrrr}2014 & 2015 & 2016 & 2017 \\ 100 & 100 & 100 & 100\end{array}$

100
2018
100

$\begin{array}{rrr}2004 & 2005 & 2 \\ 100 & 100 \\ 2019 & 2020 & 200 \\ 100 & 100 & \end{array}$

\begin{tabular}{rr}
2006 & 2007 \\
100 & 100 \\
2021 & 2022 \\
\hline 100 & 100
\end{tabular}

2008
100

$2009 \quad 2010$

End of Lifecycle Reached

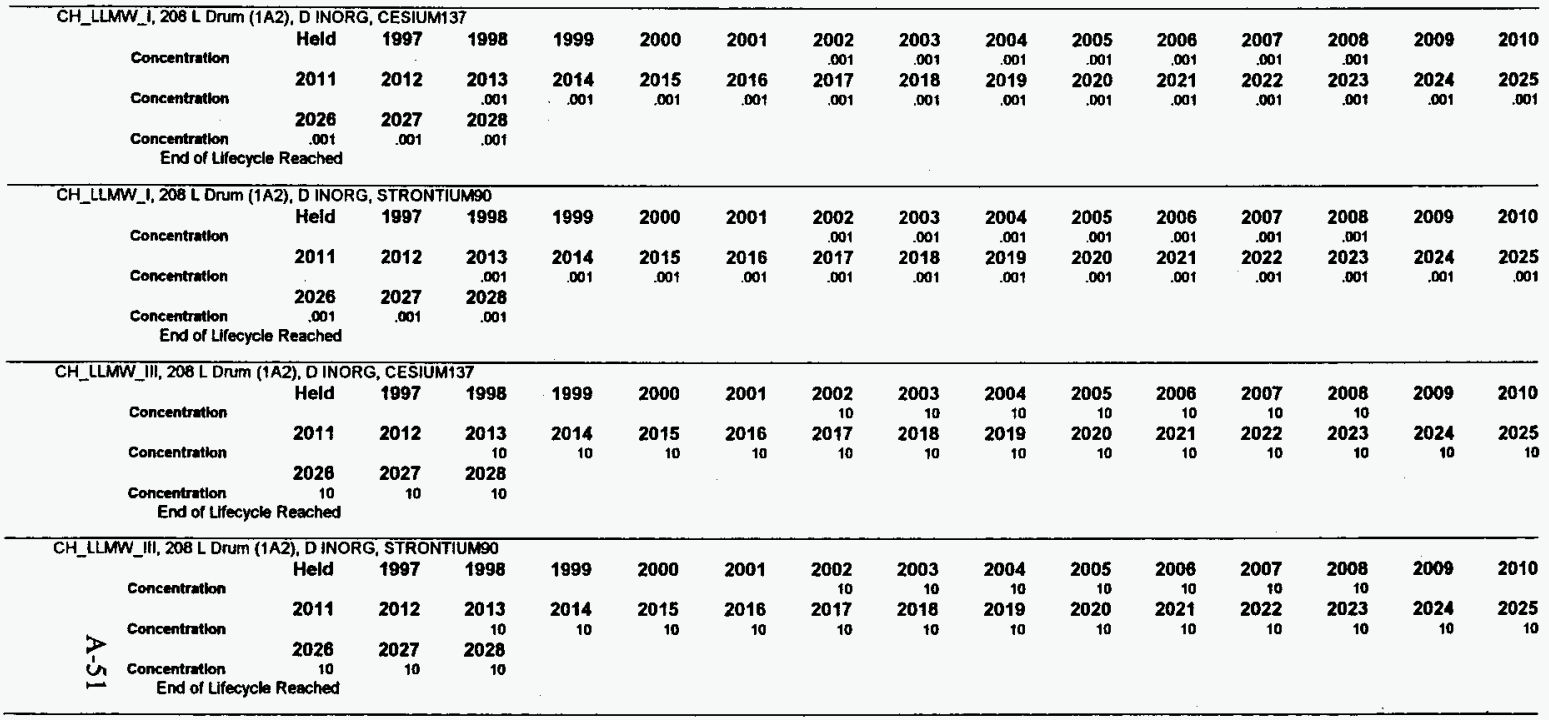


Waste Generator

Radionuclide Concentrations

( in curies per cubic meter)

Waste Class, Container, Physical Waste Form, Radionuclide

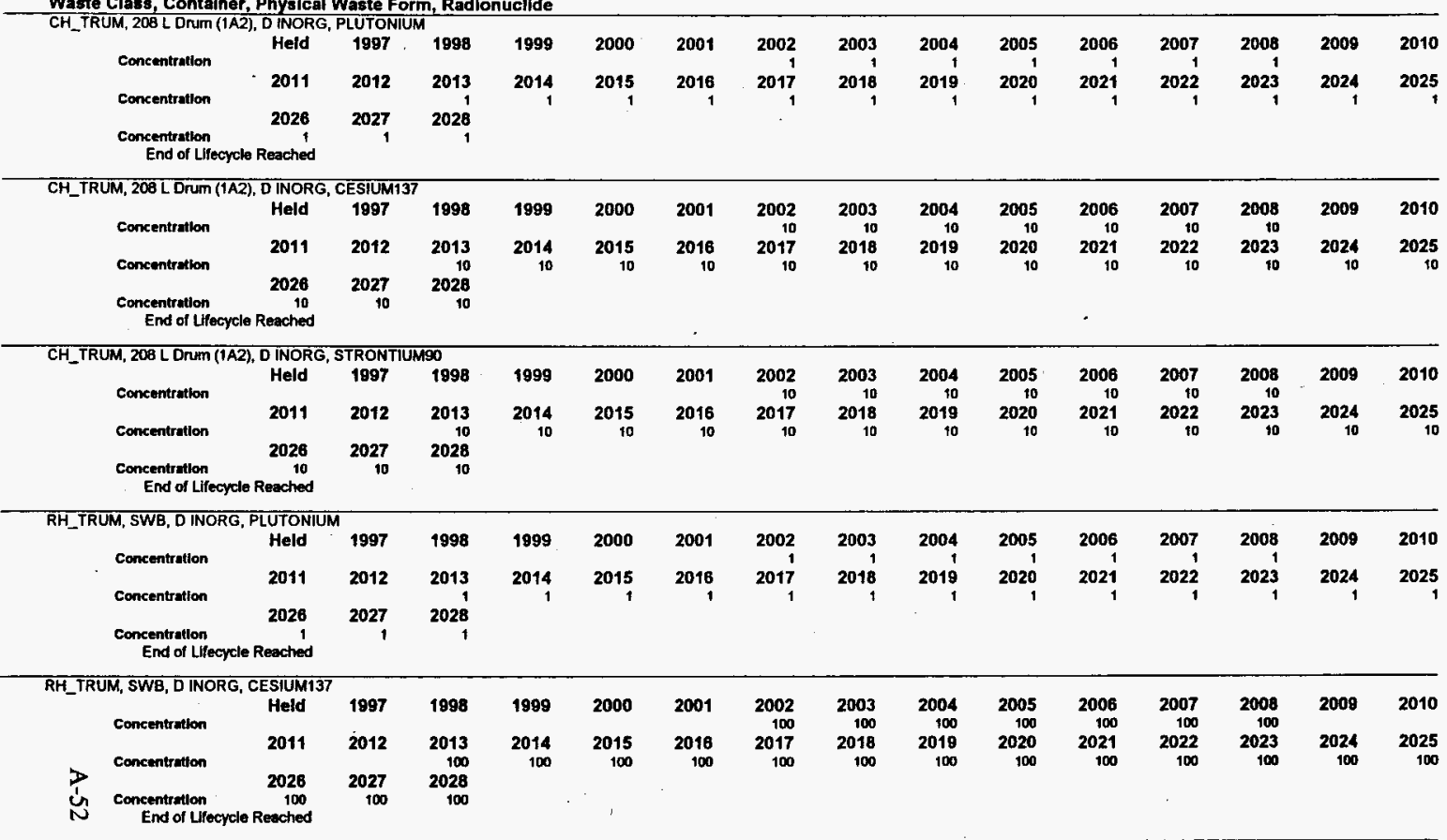


Waste Generator

Waste Class, Contalner, Physical Waste Form, Radionuclide

RH_TRUM, SWB, DTNORG, STRONTIUMGO

$\begin{array}{lllll} & \text { Held } & 1997 & 1998 & 1999\end{array}$

$\begin{array}{lllll}2011 & 2012 & 2013 & 2014\end{array}$

dionuclide Concentrations

( in curies per cubic meter)

$\begin{array}{llll}\text { Concentration } & 2028 & 2027 & 2028\end{array}$

Concentration $\quad 100$ End of Ufecycle Reached

离 
Waste Generator

Radionuciide Concentrations

Waste Class, Container, Physical Waste Form, Radionuclide WHC LLVP End Of Lifecycle: 2021

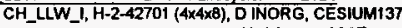

\begin{tabular}{|c|c|c|c|c|c|c|c|c|c|c|c|c|c|c|}
\hline Concentration & Held & 1997 & 1998 & 1999 & 2000 & 2001 & $\begin{array}{r}2002 \\
.001\end{array}$ & $\begin{array}{r}2003 \\
.001\end{array}$ & $\begin{array}{r}2004 \\
.001\end{array}$ & $\begin{array}{r}2005 \\
.001\end{array}$ & $\begin{array}{r}2006 \\
.001\end{array}$ & $\begin{array}{r}2007 \\
.001\end{array}$ & $\begin{array}{r}2008 \\
.001\end{array}$ & $\begin{array}{r}2009 \\
.001\end{array}$ \\
\hline oncentration & $\begin{array}{r}2011 \\
.001\end{array}$ & $\begin{array}{r}2012 \\
.001\end{array}$ & $\begin{array}{r}2013 \\
.001\end{array}$ & $\begin{array}{r}2014 \\
.001\end{array}$ & $\begin{array}{r}2015 \\
.001\end{array}$ & $\begin{array}{r}2016 \\
.001\end{array}$ & $\begin{array}{r}2017 \\
.001\end{array}$ & $\begin{array}{r}2018 \\
.001\end{array}$ & $\begin{array}{r}2019 \\
.001\end{array}$ & $\begin{array}{r}2020 \\
.001\end{array}$ & $\begin{array}{r}2021 \\
.001\end{array}$ & & & \\
\hline
\end{tabular}

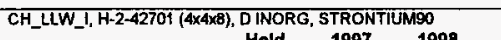

Concentration

Held 1997

$1999 \quad 2000$

Concentrution

2013

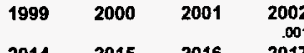

$20032004 \quad 2005$

$\begin{array}{rrrrrr}2005 & 2006 & 2007 & 2008 & 2009 & 2010 \\ 001 & 001 & 001 & 001 & .001 & .001\end{array}$

.001
End of Lifecycle Reached

CH_LLW_III, H-2-42701 (4x4x8), D INORG, CESIUM137

\begin{tabular}{|c|c|c|c|c|c|c|c|c|c|c|c|c|c|c|c|}
\hline \multicolumn{16}{|c|}{ III, H-2-42701 (4x4x8), D INORG, CESIUM137 } \\
\hline Concentration & Held & 1997 & 1998 & 1999 & 2000 & 2001 & $\begin{array}{r}2002 \\
10\end{array}$ & $\begin{array}{r}2003 \\
10\end{array}$ & $\begin{array}{r}2004 \\
10\end{array}$ & $\begin{array}{r}2005 \\
10\end{array}$ & $\begin{array}{r}2006 \\
10\end{array}$ & $\begin{array}{r}2007 \\
\text { t0 }\end{array}$ & $\begin{array}{r}2008 \\
10\end{array}$ & $\begin{array}{r}2009 \\
10\end{array}$ & $\begin{array}{r}2010 \\
10\end{array}$ \\
\hline Concentration & $\begin{array}{r}2011 \\
10 \\
10\end{array}$ & $\begin{array}{r}2012 \\
10\end{array}$ & $\begin{array}{r}2013 \\
10\end{array}$ & $\begin{array}{r}2014 \\
10\end{array}$ & $\begin{array}{r}2015 \\
10\end{array}$ & $\begin{array}{r}2016 \\
10\end{array}$ & $\begin{array}{r}2017 \\
10\end{array}$ & $\begin{array}{r}2018 \\
10\end{array}$ & $\begin{array}{r}2019 \\
10\end{array}$ & $\begin{array}{r}2020 \\
10\end{array}$ & $\begin{array}{r}2021 \\
10\end{array}$ & & & & \\
\hline
\end{tabular}

End of Litecycle Reached

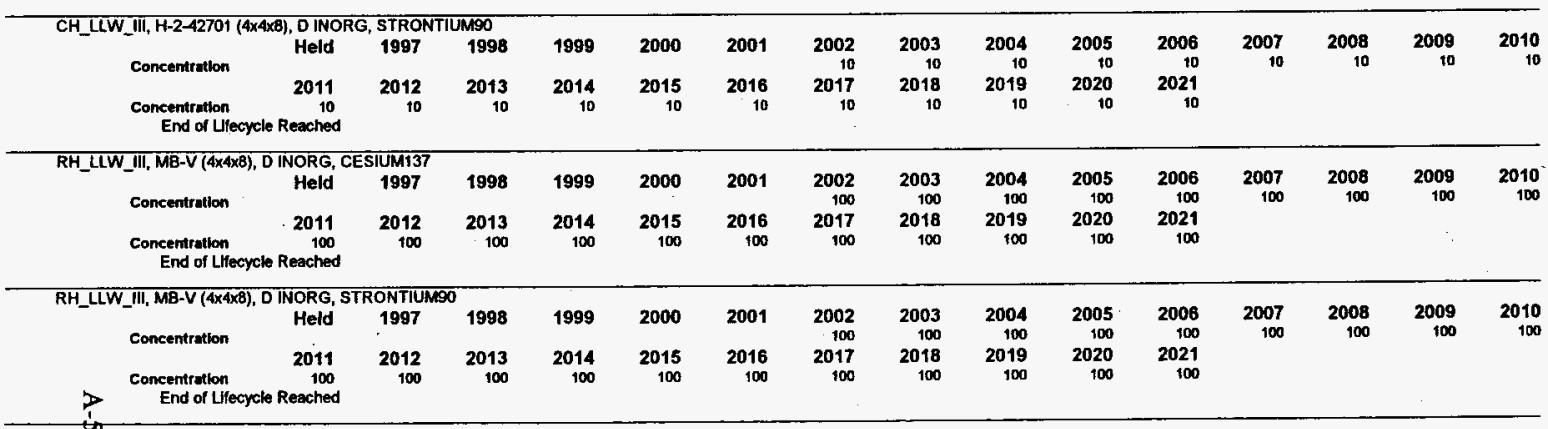


Waste Generator

Radionuclide Concentrations

(in curies per cubic meter)

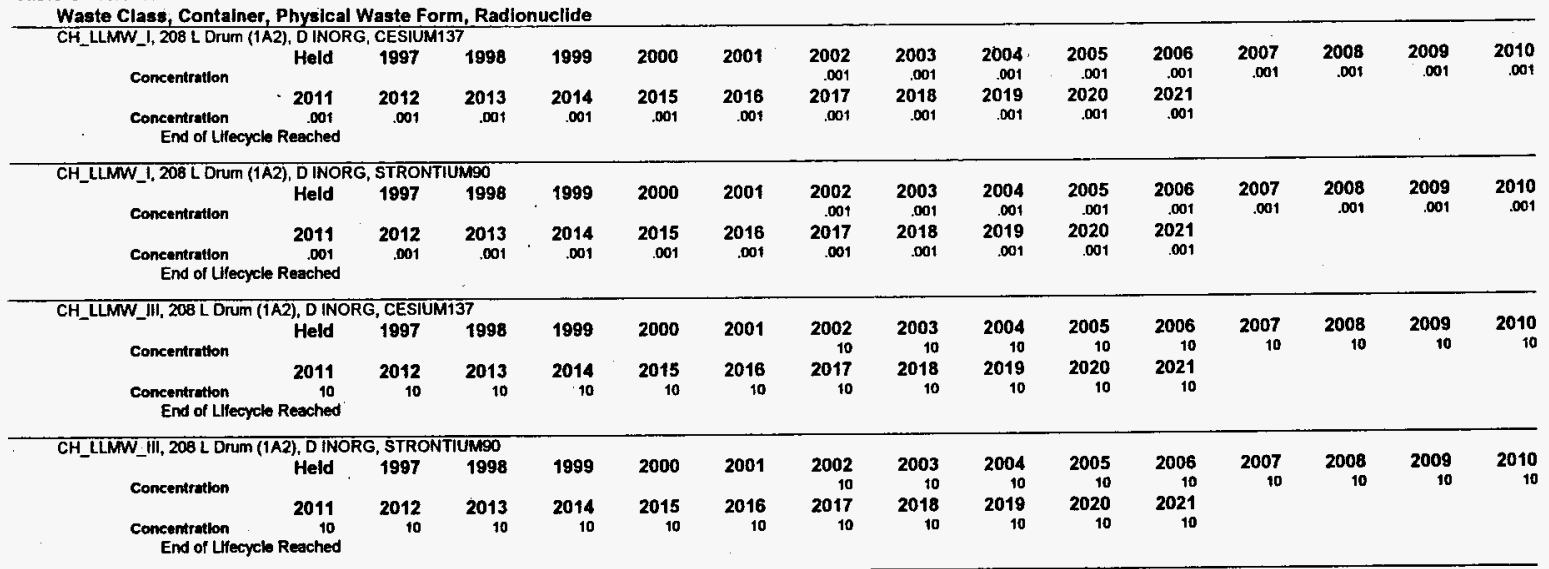


Waste Generator

Radionuclide Concentrations

Waste Class, Container, Physical Waste Form, Radlonuclide

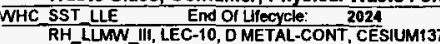

\begin{tabular}{|c|c|c|c|c|c|c|c|c|c|c|c|c|c|c|}
\hline $\begin{array}{l}\text { Concentration } \\
\text { Concentration } \\
\text { End of Lifecycte }\end{array}$ & $\begin{array}{c}\text { Held } \\
2011 \\
100 \\
\text { Reached }\end{array}$ & $\begin{array}{r}1997 \\
2012 \\
100\end{array}$ & $\begin{array}{r}1998 \\
2013 \\
100\end{array}$ & $\begin{array}{r}1999 \\
2014 \\
100\end{array}$ & $\begin{array}{r}2000 \\
2015\end{array}$ & $\begin{array}{r}2001 \\
100 \\
2016\end{array}$ & $\begin{array}{r}2002 \\
100 \\
2017\end{array}$ & $\begin{array}{r}2003 \\
100 \\
2018\end{array}$ & $\begin{array}{r}2004 \\
100 \\
2019\end{array}$ & $\begin{array}{r}2005 \\
100 \\
2020\end{array}$ & $\begin{array}{r}2006 \\
100 \\
2021\end{array}$ & $\begin{array}{r}2007 \\
100 \\
2022\end{array}$ & $\begin{array}{r}2008 \\
100 \\
2023\end{array}$ & $\begin{array}{r}2009 \\
100 \\
2024\end{array}$ \\
\hline 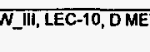 & Held & 1997 & 1998 & 1999 & 2000 & 2001 & 2002 & 2003 & 2004 & 2005 & 2006 & 2007 & 2008 & 2009 \\
\hline
\end{tabular}

\begin{tabular}{|c|c|c|c|c|c|c|c|c|c|c|c|c|c|c|}
\hline 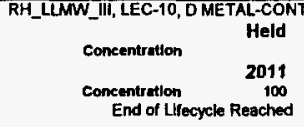 & $\begin{array}{l}\text { STRONT } \\
1997 \\
2012 \\
100\end{array}$ & $\begin{array}{l}190 \\
1998 \\
2013 \\
100\end{array}$ & $\begin{array}{r}1999 \\
2014 \\
100\end{array}$ & $\begin{array}{l}2000 \\
2015\end{array}$ & $\begin{array}{r}2001 \\
100 \\
2016\end{array}$ & $\begin{array}{r}2002 \\
100 \\
2017\end{array}$ & $\begin{array}{r}2003 \\
100 \\
2018\end{array}$ & $\begin{array}{r}2004 \\
100 \\
2019\end{array}$ & $\begin{array}{r}2005 \\
100 \\
2020\end{array}$ & $\begin{array}{r}2006 \\
100 \\
2021\end{array}$ & $\begin{array}{r}2007 \\
100 \\
2022\end{array}$ & $\begin{array}{r}2008 \\
100 \\
2023\end{array}$ & $\begin{array}{r}2009 \\
100 \\
2024\end{array}$ & $\begin{array}{r}2010 \\
100\end{array}$ \\
\hline $\begin{array}{cc}\text { RH_LLMW_fil, LEC-11, D METAL-CONT } & \text { Held } \\
\text { Concentration } & 2011 \\
\text { Concentration } & 100 \\
\text { End of Lifecycle Reached }\end{array}$ & $\begin{array}{l}\text { CESIUM1 } \\
1997 \\
2012 \\
100\end{array}$ & $\begin{array}{r}1998 \\
2013 \\
100\end{array}$ & $\begin{array}{r}1999 \\
2014 \\
100\end{array}$ & $\begin{array}{l}2000 \\
2015\end{array}$ & $\begin{array}{r}2001 \\
100 \\
2016\end{array}$ & $\begin{array}{r}2002 \\
100 \\
2017\end{array}$ & $\begin{array}{r}2003 \\
100 \\
2018\end{array}$ & $\begin{array}{r}2004 \\
100 \\
2019\end{array}$ & $\begin{array}{r}2005 \\
100 \\
2020\end{array}$ & $\begin{array}{r}2006 \\
100 \\
2021\end{array}$ & $\begin{array}{r}2007 \\
100 \\
2022\end{array}$ & $\begin{array}{r}2008 \\
100 \\
2023\end{array}$ & $\begin{array}{r}2009 \\
100 \\
2024\end{array}$ & $\begin{array}{r}2010 \\
100\end{array}$ \\
\hline $\begin{array}{cc}\text { RH_LLWW_IIT, LEC-11, D METAL-CONT } & \text { Held } \\
\text { Concentration } & 2011 \\
& 100 \\
\text { Concentration } & 100 \\
\text { End of Lifecycle Reached }\end{array}$ & $\begin{array}{l}\text { STRONT } \\
1997 \\
2012 \\
100\end{array}$ & $\begin{array}{l}190 \\
1998 \\
2013 \\
100\end{array}$ & $\begin{array}{r}1999 \\
2014 \\
100\end{array}$ & $\begin{array}{l}2000 \\
2015\end{array}$ & $\begin{array}{r}2001 \\
100 \\
2016\end{array}$ & $\begin{array}{r}2002 \\
100 \\
2017\end{array}$ & $\begin{array}{r}2003 \\
100 \\
2018\end{array}$ & $\begin{array}{r}2004 \\
100 \\
2019\end{array}$ & $\begin{array}{r}2005 \\
100 \\
2020\end{array}$ & $\begin{array}{r}2006 \\
100 \\
2021\end{array}$ & $\begin{array}{r}2007 \\
100 \\
2022\end{array}$ & $\begin{array}{r}2008 \\
100 \\
2023\end{array}$ & $\begin{array}{r}2009 \\
100 \\
2024\end{array}$ & $\begin{array}{r}2010 \\
100\end{array}$ \\
\hline $\begin{array}{cc}\text { RH_LLMW_III, LEC-12, O METAL-CONT } & \text { Held } \\
\text { Concentration } & \\
& 2011 \\
\text { Concentration } & 100 \\
\text { End of Lifecycle Reached }\end{array}$ & $\begin{array}{l}\text { ESIUM1 } \\
1997 \\
2012 \\
100\end{array}$ & $\begin{array}{r}1998 \\
2913 \\
100\end{array}$ & $\begin{array}{r}1899 \\
2014 \\
100\end{array}$ & $\begin{array}{r}2000 \\
2015 \\
100\end{array}$ & $\begin{array}{r}2001 \\
100 \\
2016 \\
100\end{array}$ & $\begin{array}{r}2002 \\
100 \\
2017 \\
100\end{array}$ & $\begin{array}{l}2003 \\
2018\end{array}$ & $\begin{array}{r}2004 \\
100 \\
2019\end{array}$ & $\begin{array}{r}2005 \\
100 \\
2020\end{array}$ & $\begin{array}{r}2006 \\
100 \\
2021\end{array}$ & $\begin{array}{r}2007 \\
100 \\
2022\end{array}$ & $\begin{array}{r}2008 \\
100 \\
2023\end{array}$ & $\begin{array}{r}2009 \\
100 \\
2024\end{array}$ & $\begin{array}{r}2010 \\
100\end{array}$ \\
\hline 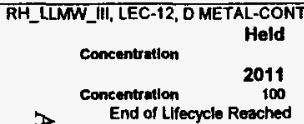 & $\begin{array}{r}\text { STRONT } \\
1997 \\
2012 \\
100\end{array}$ & $\begin{array}{l}1900 \\
1998 \\
2013 \\
100\end{array}$ & $\begin{array}{r}1999 \\
2014 \\
100\end{array}$ & $\begin{array}{r}2000 \\
2015 \\
100\end{array}$ & $\begin{array}{r}2001 \\
100 \\
2016 \\
100\end{array}$ & $\begin{array}{r}2002 \\
100 \\
2017 \\
100\end{array}$ & $\begin{array}{l}2003 \\
2018\end{array}$ & $\begin{array}{r}2004 \\
100 \\
2019\end{array}$ & $\begin{array}{r}2005 \\
100 \\
2020\end{array}$ & $\begin{array}{r}2006 \\
100 \\
2021\end{array}$ & $\begin{array}{r}2007 \\
100 \\
2022\end{array}$ & $\begin{array}{r}2008 \\
500 \\
2023\end{array}$ & $\begin{array}{r}2009 \\
100 \\
2024\end{array}$ & $\begin{array}{r}2010 \\
100\end{array}$ \\
\hline
\end{tabular}


Waste Class, Contalner, Physical Waste Form, Radionuclide

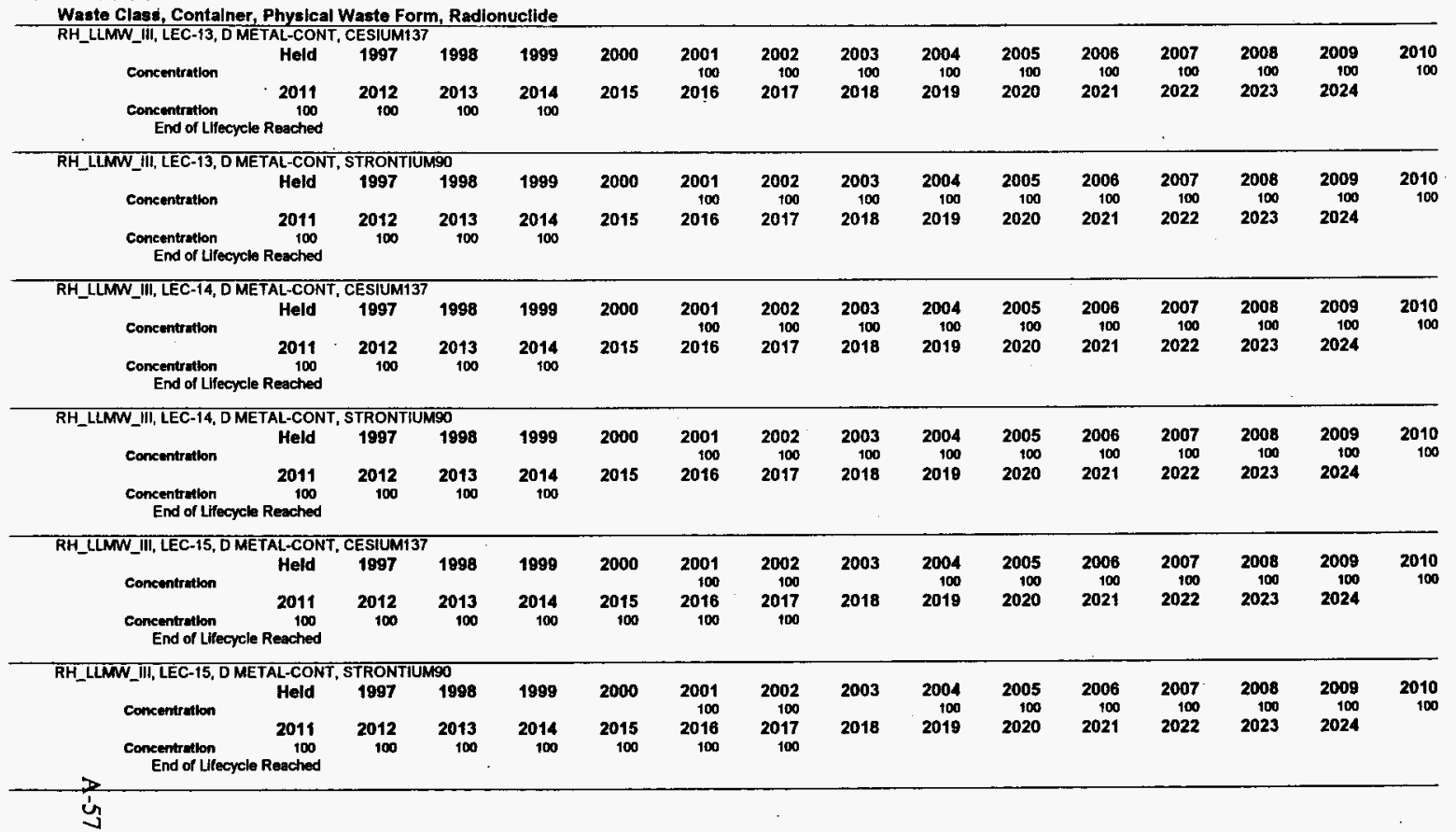




\section{Vaste Generator}

(In curles per cublic meter)

Waste Class, Container, Physical Waste Form, Radionuclide

RH_LLMW_III, LEC-16, D METAL-CONT, CESIUM137

\begin{tabular}{|c|c|c|c|c|c|c|c|c|c|c|c|c|c|c|c|}
\hline $\begin{array}{l}\text { Concentrution } \\
\text { Concentration } \\
\text { End of Lifec }\end{array}$ & $\begin{array}{l}\text { Held } \\
2011 \\
\text { Reached }\end{array}$ & $\begin{array}{l}1997 \\
2012\end{array}$ & $\begin{array}{l}1998 \\
2013\end{array}$ & $\begin{array}{l}1999 \\
2014\end{array}$ & $\begin{array}{l}2000 \\
2015\end{array}$ & $\begin{array}{r}2001 \\
100 \\
2016\end{array}$ & $\begin{array}{r}2002 \\
100 \\
2017\end{array}$ & $\begin{array}{l}2003 \\
2018\end{array}$ & $\begin{array}{l}2004 \\
2019\end{array}$ & $\begin{array}{l}2005 \\
2020\end{array}$ & $\begin{array}{l}2006 \\
2021\end{array}$ & $\begin{array}{l}2007 \\
2022\end{array}$ & $\begin{array}{l}2008 \\
2023\end{array}$ & $\begin{array}{l}2009 \\
2024\end{array}$ & 2010 \\
\hline Concentration & $\begin{array}{l}\text { AL-CON } \\
\text { Held } \\
2011\end{array}$ & $\begin{array}{c}\text { STRONT } \\
1997 \\
2012\end{array}$ & $\begin{array}{l}1998 \\
190 \\
2013\end{array}$ & $\begin{array}{l}1999 \\
2014\end{array}$ & $\begin{array}{l}2000 \\
2015\end{array}$ & $\begin{array}{r}2001 \\
100 \\
2016\end{array}$ & $\begin{array}{r}2002 \\
100 \\
2017\end{array}$ & $\begin{array}{l}2003 \\
2018\end{array}$ & $\begin{array}{l}2004 \\
2019\end{array}$ & $\begin{array}{l}2005 \\
2020\end{array}$ & $\begin{array}{l}2006 \\
2021\end{array}$ & $\begin{array}{l}2007 \\
2022\end{array}$ & $\begin{array}{l}2008 \\
2023\end{array}$ & $\begin{array}{r}2009 \\
2024\end{array}$ & 2010 \\
\hline
\end{tabular}

Concentration

End of Lifecycte Reached

\begin{tabular}{|c|c|c|c|c|c|c|c|c|c|c|c|c|c|c|}
\hline $\begin{array}{l}\text { RH_LLMW_III, LEC-18, D METAL-CON } \\
\text { Held } \\
\text { Concentration } \\
\text { Concentration } \\
\text { End of Lifecycle Reached }\end{array}$ & $\begin{array}{c}\text { CESIUM } \\
1997 \\
2012\end{array}$ & $\begin{array}{r}1998 \\
2013\end{array}$ & $\begin{array}{l}1989 \\
2014\end{array}$ & $\begin{array}{l}2000 \\
2015\end{array}$ & $\begin{array}{l}2001 \\
2016\end{array}$ & $\begin{array}{l}2002 \\
2017\end{array}$ & $\begin{array}{l}2003 \\
2018\end{array}$ & $\begin{array}{l}2004 \\
2019\end{array}$ & $\begin{array}{l}2005 \\
2020\end{array}$ & $\begin{array}{l}2006 \\
2021\end{array}$ & $\begin{array}{l}2007 \\
2022\end{array}$ & $\begin{array}{l}2008 \\
2023\end{array}$ & $\begin{array}{r}2009 \\
100 \\
2024\end{array}$ & 2010 \\
\hline $\begin{array}{l}\text { RH_LLMW_III, LEC } \bar{C}-18,0 \text { METAL-CON } \\
\text { Held } \\
\text { Concentration } \\
\text { Concentration } \\
\text { End of Lifecycle Reached }\end{array}$ & $\begin{array}{c}\text { STRONT } \\
1997 \\
2012 \\
\end{array}$ & $\begin{array}{l}190 \\
1998 \\
2013\end{array}$ & $\begin{array}{l}1999 \\
2014\end{array}$ & $\begin{array}{l}2000 \\
2015\end{array}$ & $\begin{array}{l}2001 \\
2016\end{array}$ & $\begin{array}{l}2002 \\
2017\end{array}$ & $\begin{array}{l}2003 \\
2018\end{array}$ & $\begin{array}{l}2004 \\
2019\end{array}$ & $\begin{array}{l}2005 \\
2020\end{array}$ & $\begin{array}{l}2006 \\
2021\end{array}$ & $\begin{array}{l}2007 \\
2022\end{array}$ & $\begin{array}{l}2008 \\
2023\end{array}$ & $\begin{array}{r}2009 \\
100 \\
2024\end{array}$ & 2010 \\
\hline $\begin{array}{c}\text { CH_TRUM, LEC-16, DMETAL-CONT, } \mathrm{P} \\
\text { Held } \\
\text { Concentration } \\
\text { Concentration } \\
\text { End of Lifecycle Reached }\end{array}$ & $\begin{array}{l}\text { JTONIUN } \\
1997 \\
2012\end{array}$ & $\begin{array}{l}1998 \\
2013\end{array}$ & $\begin{array}{l}1999 \\
2014\end{array}$ & $\begin{array}{l}2000 \\
2015\end{array}$ & $\begin{array}{r}2001 \\
1 \\
2016\end{array}$ & $\begin{array}{r}2002 \\
1 \\
2017\end{array}$ & $\begin{array}{r}2003 \\
1 \\
2018\end{array}$ & $\begin{array}{l}2004 \\
2019\end{array}$ & $\begin{array}{l}2005 \\
2020\end{array}$ & $\begin{array}{l}2006 \\
2021\end{array}$ & $\begin{array}{l}2007 \\
2022\end{array}$ & $\begin{array}{l}2008 \\
2023\end{array}$ & $\begin{array}{l}2009 \\
2024\end{array}$ & 2010 \\
\hline $\begin{array}{c}\text { CH_TRUM, LEC-16, D METAL-CONT, C } \\
\text { Concentration } \\
\text { Coneentration } \\
\text { End of Lfecycle Reached }\end{array}$ & $\begin{array}{c}\text { SIUM137 } \\
1997 \\
2012\end{array}$ & $\begin{array}{l}1998 \\
2043\end{array}$ & $\begin{array}{r}1999 \\
2014\end{array}$ & $\begin{array}{l}2000 \\
2015\end{array}$ & $\begin{array}{r}2001 \\
10 \\
2016\end{array}$ & $\begin{array}{r}2002 \\
10 \\
2017\end{array}$ & $\begin{array}{r}2003 \\
10 \\
2018\end{array}$ & $\begin{array}{c}2004 \\
2019\end{array}$ & $\begin{array}{r}2005 \\
2020\end{array}$ & $\begin{array}{l}2006 \\
2021\end{array}$ & $\begin{array}{l}2007 \\
2022\end{array}$ & $\begin{array}{l}2008 \\
2023\end{array}$ & $\begin{array}{l}2009 \\
2024\end{array}$ & 2010 \\
\hline
\end{tabular}


Waste Generator

Radionuclide Concentrations

(in curies per cubic meter)

Waste Class, Container, Physical Waste Form, Radionuclide

CH_TRUM, LEC-16, D METAL-CONT, STRONTIUM9O

coneentration Held 1997

$1998 \quad 1999 \quad 2000$ $\begin{array}{lllll}2011 & 2012 & 2013 & 2014 & 2015\end{array}$

2001

2001 2002 2003

2004

10
2017

10
2018

$2018 \quad 2019$

20192020

$2020 \quad 2021$

$2021 \quad 2022$

$2022 \quad 2023$

20232024

End of Lilfecycle Reached .

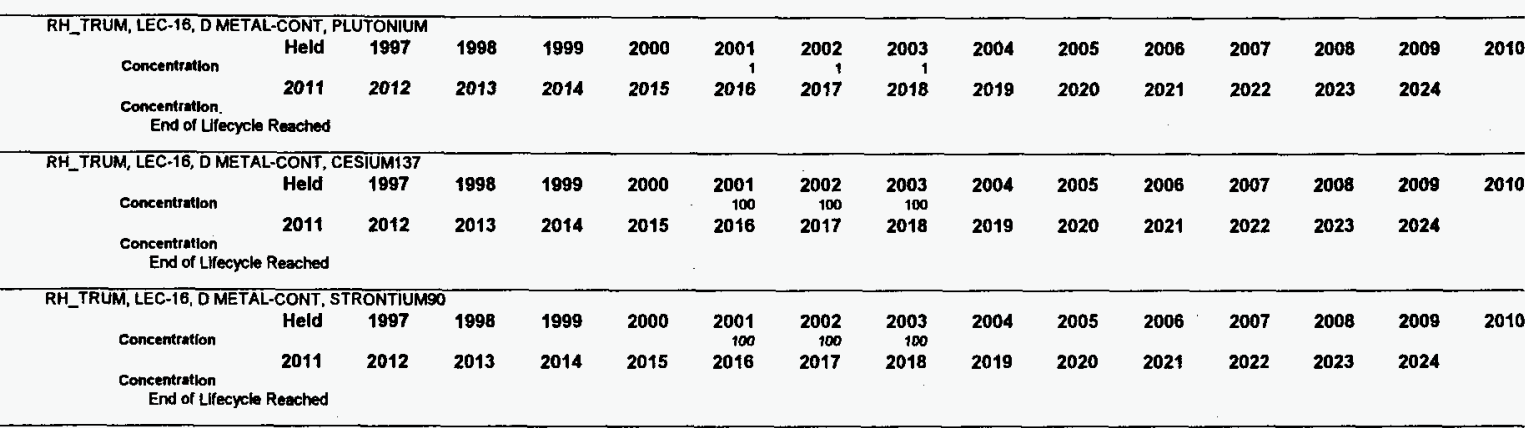


Naste Generator

Radionuclide Concentrations

Page: 11

( In curies per cubic meter)

NiC Waste Form, Radionuclide

\begin{tabular}{c} 
WHC SST RET \\
\hline CH_LLW_I. 208 L Drum (1A1), D INORG, CESIUM 137 \\
Heid
\end{tabular}

\begin{tabular}{|c|c|c|c|c|c|c|c|c|c|c|c|c|c|c|c|}
\hline Concentration & Heid & 1997 & & & & & & & $\begin{array}{r}2004 \\
.009\end{array}$ & $\begin{array}{r}2005 \\
.001\end{array}$ & $\begin{array}{r}2006 \\
.001\end{array}$ & $\begin{array}{r}2007 \\
.001\end{array}$ & $\begin{array}{r}2008 \\
.001\end{array}$ & $\begin{array}{r}2009 \\
.001\end{array}$ & $\begin{array}{r}2010 \\
.001\end{array}$ \\
\hline $\begin{array}{l}\text { Concentration } \\
\text { End of Lifecycle }\end{array}$ & $\begin{array}{c}2011 \\
.001 \\
\text { Reached }\end{array}$ & $\begin{array}{r}2012 \\
.001\end{array}$ & $\begin{array}{r}2013 \\
.001\end{array}$ & $\begin{array}{r}2014 \\
.001\end{array}$ & $\begin{array}{r}2015 \\
.001\end{array}$ & $\begin{array}{r}2016 \\
.001\end{array}$ & $\begin{array}{r}2017 \\
.001\end{array}$ & 2018 & & & & & & & \\
\hline Concentration & Held & 1997 & $\begin{array}{l}190 \\
1998\end{array}$ & 1999 & 2000 & 2001 & 2002 & 2003 & $\begin{array}{r}2004 \\
.001\end{array}$ & $\begin{array}{r}2005 \\
.001\end{array}$ & $\begin{array}{r}2006 \\
.001\end{array}$ & $\begin{array}{r}2007 \\
.001\end{array}$ & $\begin{array}{r}2008 \\
.001\end{array}$ & $\begin{array}{r}2009 \\
.001\end{array}$ & $\begin{array}{r}2010 \\
.001\end{array}$ \\
\hline
\end{tabular}

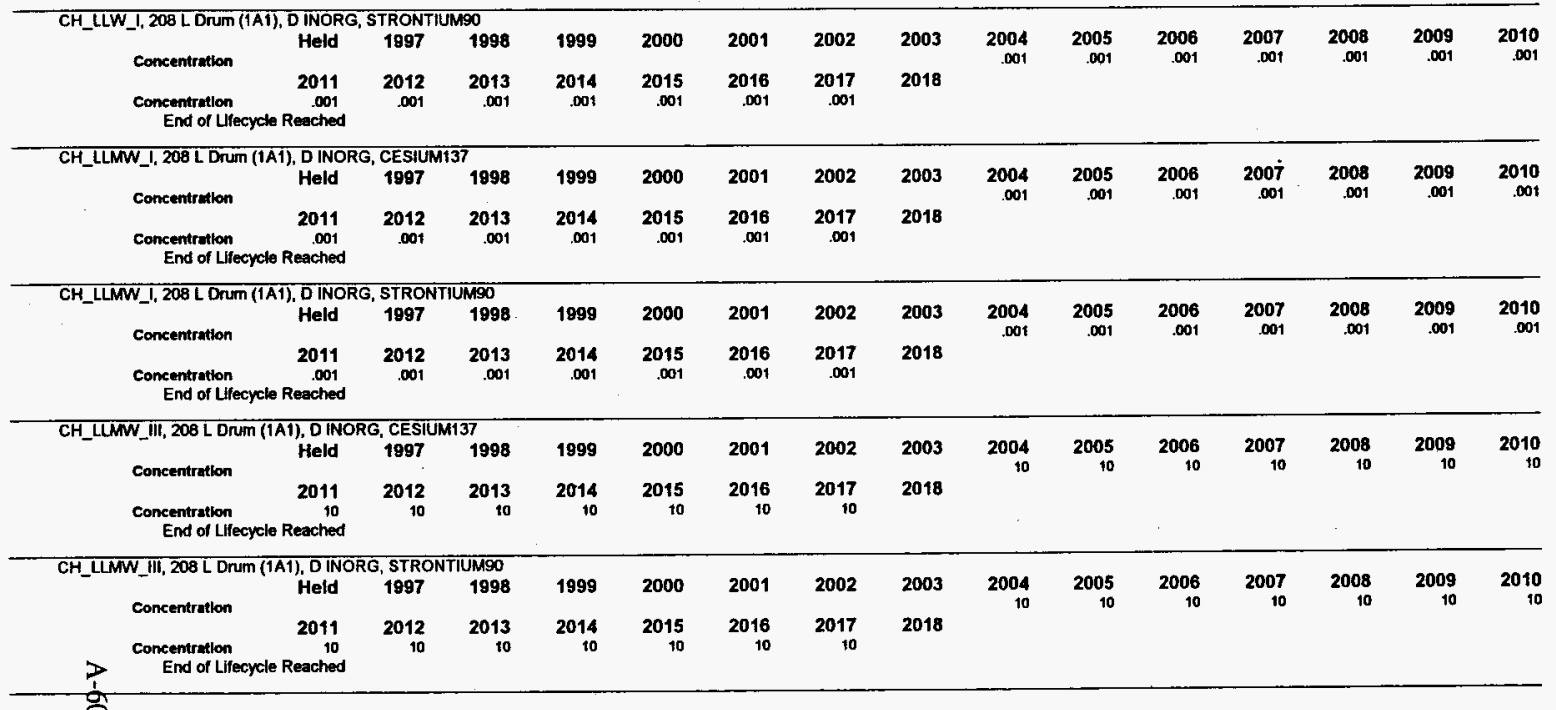


Waste Generator

Radionuclide Concentrations

( In curies per cubic meter)

Waste Class, Container, Physical Waste Form, Radionuclide

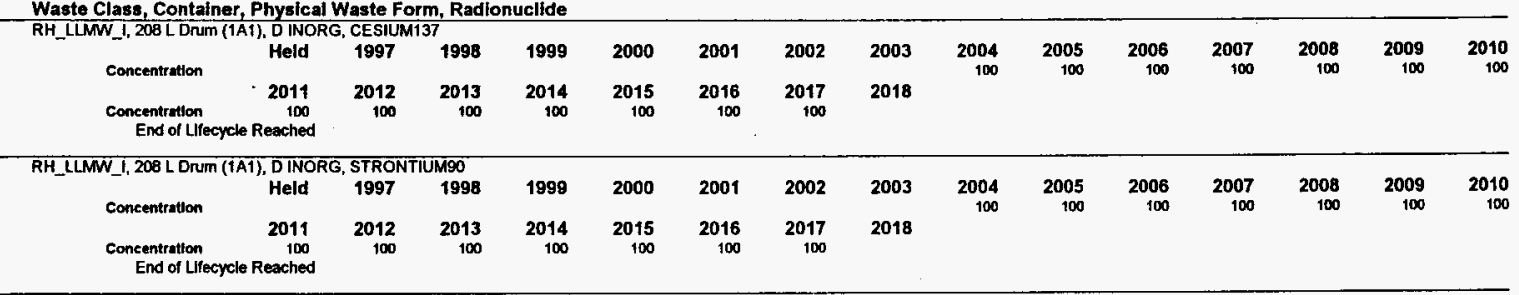


WHC-SD-WM-TI-777

Revision 0

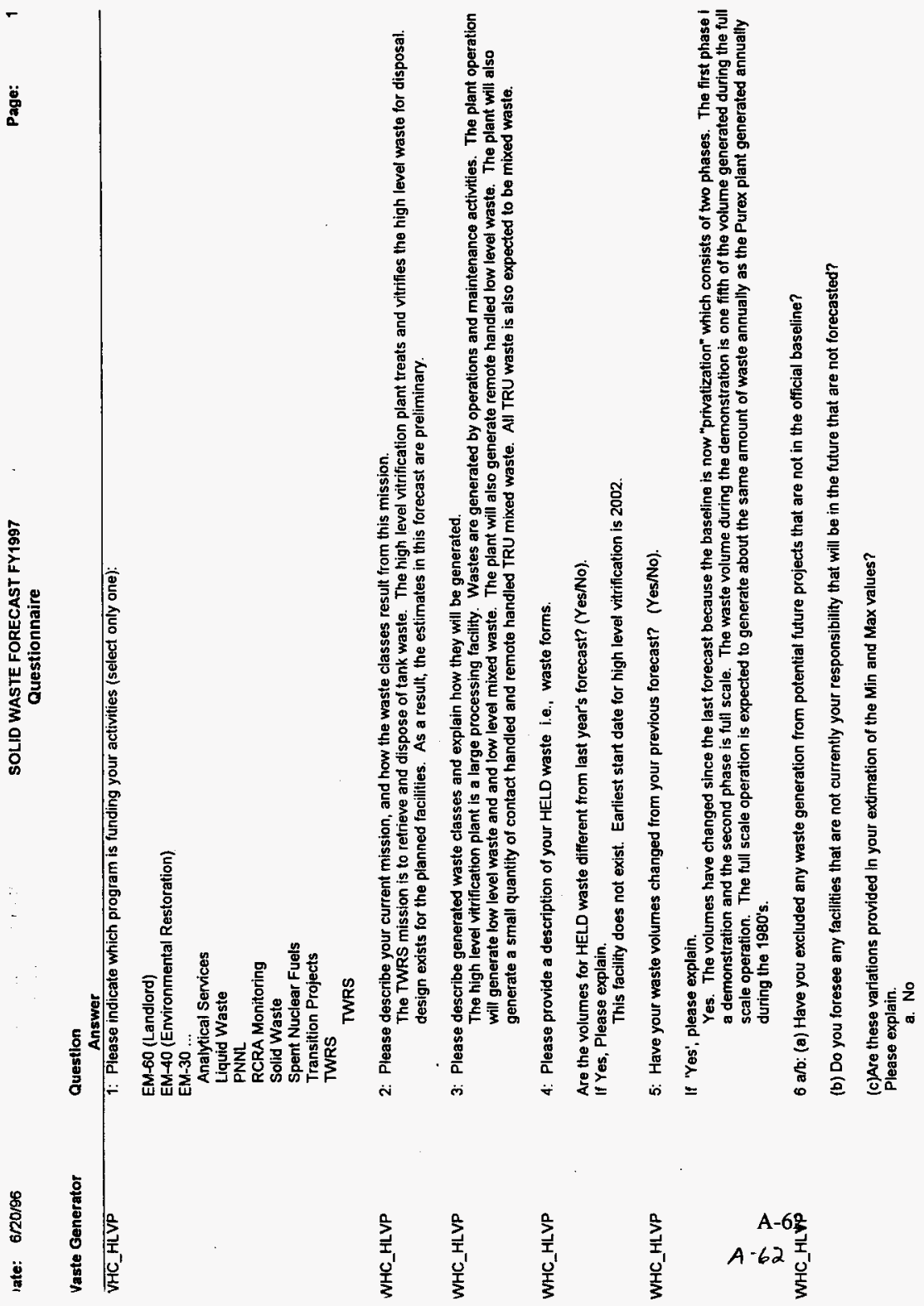




\begin{tabular}{r} 
Vaste Generator \\
\hline $\begin{array}{r}\text { Question No } \\
\text { Answer }\end{array}$ \\
c. N/A
\end{tabular}

NHC_HLVP

7: Will waste be separated into like waste forms prior to shipping?

For example, if you generate contaminated soils, inorganic particulates and metal debris, will you ship these waste in separate containers, or will the waste b combined and placed into one container? Please explain for each waste class.

Waste will generally be packaged as generated. If the wastes are generated separately, the wastes will be packaged separately.

NHC_HLVP

8 a/b: (a) Will you generate hazardous or mixed waste? (Yes/No)

If 'Yes', (b) Will you place several hazardous constituents in one container or will you segregate the constituents priot to shipping?

That is, If you generate toxic metals and ignitables, will you ship these constituents in separate containers or will they be combined and placed into one container?

a. Yes

b. Wastes contaminated by tank waste may contain hazardous constituents. Segregation will be used to maximum extent possible.

9: Please describe your quarterly expected shipping schedule for each applicable waste class during FY 1997.

For example: $40 \%$ of waste shipped during the first quarter, $20 \%$ shipped in the second quarter, $40 \%$ shipped in the fourth quarter N/A. This facility does not start until 2002 or later.

10: Discuss the assumptions that were used to prepare this forecast

The demonstration plant begins in 2002 and operates through 2008. The full scale plant begins in 2013 and nuns through 2028. All of the tani waste is retrieved and processed. The schedule is based on the TWRS Disposal Program OWVP for March 1, 1996, updated May 14, 1996.

11: Please list any Tri-Party Agreernent (TPA) milestones that affect your facility/program.

For each TPA milestone please indicate your status in achieving the milestone and whether these milestones are reflected in your forecast.

The related milestones are discussed in a memorandum from John Wagoner to the Department of Ecology, "Completion of Negotiations of Hanfor Federal Facility Agreement and Consent Order Milestones M-50-00, M-5i-00, and M-60-00," dated December 21, 1995.

12: What assumptions were used in estimating the minimum and maximum waste percentages identified in Table 1?

For example, the minimum waste $\%$ is based upon the majority of the waste being sent to a commercial disposal site.

The maximum waste $\%$ is based upon additional projects being initiated that will generate waste not accounted for in the best estimate volume. The waste numbers are very preliminary and were generally given a range of $50 \%$ to $200 \%$, primarily due to the uncertainty of dealing with preliminary facility and processing concepts. 


\begin{tabular}{l}
$\begin{array}{c}\text { Question } \\
\text { Answer }\end{array}$ \\
\hline VHC_HLVP \\
$\begin{array}{l}\text { 13: Please provide a description of how the radionuclides were determined? } \\
\text { For example, historical data or sample analysis data were used. } \\
\text { The radionuclide estimates are preliminary based on expected feed materials and processes. Reference WHC-SD-WM-TI-694, "Tank Waste } \\
\text { Remediation System Optimized Processing Strategy." Cesium-137 and Strontium-90 generally dominate all other radionuclides in the tank waste. }\end{array}$
\end{tabular}

NHC_HLVP

$14 \mathrm{a} / \mathrm{b} / \mathrm{c}$ : Indicate confidence levels for the following waste characterization data:

For example, the physical waste forms are based upon historical characterization information.

(a) Physical Waste Forms

High

Low

Medium

Explain:

(b) Hazardous Constituents

High

Low

Medium

Explain:

(c) Radiation Type

High

Low

Medium

Explain:

a. Meduium. The physical waste forms are based on waste historically generated by Hanford processing facilities and the knowedge of the characteristics of tank waste.

b. Medium

c. Medium 
Vaste Generator

VHC.HLVP

Question

Answer

$15 \mathrm{a} / \mathrm{b} / \mathrm{c}$ : (a) What percentage of the waste forecasted is existing waste and what percentage is future generated waste?

(b) Of your existing waste, how confident are you in the current shipping schedule? (High, Low, Medium)

Explain:

(c) Of your future generated waste, how confident are you in the volumes and shipping schedule? (High, Low, Medium)

Explain:

a. All waste is to be generated in the future.

b. N/A

c. Low. Schedules are subject to change due to contracting uncertainties at Hanford and with the privatized contracts.

NHC_HLVP

$16 \mathrm{a} / \mathrm{b} / \mathrm{c}$ : Indicate whether your forecast includes: operational, terminal clean-out \& stabilization (TC\&S) ,and/or D\&D wastes.

Does this forecast include:

(a) Operational waste?

(b) Terminal clean-out \& stabilization waste (TC\&S)?

(c) Decontamination \& Decommissioning waste (D\&D)?

a. Yes

b. No

c. No

17: If you have not included TC\&S or D\&D waste, will this waste potentially be managed at Hanford? (Yes/No)

If 'Yes', please provide an estimate of the expected volumes.

Terminal cleanout and D\&D will be after 2008 for the demonstration facility and after 2028 for the full scale facility. How this waste is handled is unknown.

18: When will your facility transition into a terminal clean-out and stabilization mission? This is unknown.

19: When is your facility expected to be added to the Surplus Facility List? This is unknown. 


\begin{tabular}{lc} 
Vaste Generator & $\begin{array}{c}\text { Question } \\
\text { Answer }\end{array}$ \\
\hline VHC_HLVP & $20:$ Will waste
\end{tabular}

Questionnaire

20: Will waste be generated between terminal clean-out/stabilization and $\mathrm{D} \overline{\mathrm{Q}} \mathrm{D}$ ? (Yes/No)

If 'Yes', please provide a waste description and identify the organization and department that has responsibility for this close-out waste? This is unknown.

NHC HLVP

21: What document titles and numbers can be provided that give insight into solid waste generation at your facility?

Please provide a tist of document tities and document numbers that provide insight into solid waste generated during operations, terminal clean-out and stabilization activities and or D\&D activities.

Reference WHC-SD-WM-TI-694, "Tank Waste Remediation System Optimized Processing Strategy."

NHC_HLVP

22: Does your facility have an approved Site Treatment Plan (STP) or a Proposed Site Treatment Plan (PSTP) in progress?

NOTE - OFFSITE GENERATORS ONLY, re: Richfand Operations Office Letter (RL) \#95-SWT-174, Guidance for Acceptance of Offsite Mixed Waste, Not applicable

WHC_LLVP
1: Please indicate which program is
EM-60 (Landlord)
EM-40 (Environmental Restoration)
EM-30 ...
Analytical Services
Liquid Waste
PNNL
RCRA Monitoring
Solid Waste
Spent Nuclear Fuels
Transition Projects
TWRS TWRS

WHC LLVP

WHC LLYE
$\frac{1}{2}$
$\frac{1}{1}$

WHC_LLVP T)

2: Please describe your current mission, and how the waste classes result from this mission

The TWRS mission is to retrieve and dispose of tank waste. The low level vitrification plant treats and vitrifies the low levet waste for disposal. No design exists for the planned facilities. As a result, the estimates in this forecast are preliminary.

3: Please describe generated waste classes and explain how they will be generated.

The low level vitrification plant is a large processing facility even larger than the high level vitrification plant. Wastes are generated by operations and maintenance activities. The plant operation will generate low level waste and and low level mixed waste. The plant will also generate remote handled low level waste. The plant is not expected to generate TRU waste.

4: Please provide a description of your HELD waste i.e., waste forms.

Are the volumes for HELD waste difierent from last year's forecast? (Yes/No)

If Yes, Please explain.

This facility does not exist. Earliest start date for low level vitrification is $\mathbf{2 0 0 2}$ 


\section{Waste Generator Question}

Answer

WHC_LLVP $\quad 5$ : Have your waste volumes changed from your previous forecast? (Yes/No).

If 'Yes', please explain.

Yes. The volumes have changed since the last forecast because the baseline is now "privatization" which consists of two phases. The first phase $i$ a demonstration and the second phase is full scale. The waste volume during the demonstration is one fifth of the volume generated during the full scale operation. The full scale operation is expected to generate about twice the amount of waste annually as the Purex plant generated annually during the 1980 's. The previous forecast included a pretreatment facility. Pretreatment is no longer a separate facility. Required pretreatment is included in the vitrification facility.

WHC_LLVP

6 a/b: (a) Mave you excluded any waste generation from potential future projects that are not in the official baseline?

(b) Do you foresee any facilities that are not currently your responsibility that will be in the future that are not forecasted?

(c) Are these variations provided in your extimation of the Min and Max values? Please explain.
a. No
b. No
c. N/A

7: Will waste be separated into like waste forms prior to shipping?

For example, if you generate contaminated soils, inorganic particulates and metal debris, will you ship these waste in separate containers, or will the waste b combined and placed into one container?

Please explain for each waste class.

Waste will generaily be packaged as generated. If the wastes are generated separately, the wastes will be packaged separately.

WHC_LLVP

$8 \mathrm{a} / \mathrm{b}$ : (a) Will you generate hazardous or mixed waste? (Yes/No)

If 'Yes', (b) Will you place several hazardous constituents in one container or will you segregate the constituents prior to shipping?

That is, if you generate toxic metals and ignitables, will you ship these constituents in separate containers or will they be combined and placed into one container?

a. Yes

b. Wastes contaminated by tank waste may contain hazardous constituents. Segregation will be used to maximum extent possible. 
Waste Generator

WHC_LLVP

WHC_LLVP

WHC_LLVP

WHC_LLVP

WHC_LLVP

$\begin{array}{ll} & \begin{array}{l}\text { For exam } \\ \text { (a) Physica }\end{array} \\ & \text { High } \\ & \text { Low } \\ & \text { Medium } \\ \coprod_{\infty} & \text { Explain: }\end{array}$

(b) Hazardous Constituents

High

Low

Medium

Answer

Physical Waste Forms

Medium

\section{Questionnaire}

10: Discuss the assumptions that were used to prepare this forecast.

The demonstration plant begins in 2002 and operates through 2011. The full scale plant begins in 2011 and runs through 2021. All of the tank waste is retrieved and processed. The schedule is based on the TWRS Disposal Program OWVP for March 1, 1996, updated May 14, 1996.

11: Please list any Tri-Party Agreement (TPA) milestones that affect your facility/program.

For each TPA milestone please indicate your status in achieving the milestone and whether these milestones are reflected in your forecast.

The related milestones are discussed in a memorandum from John Wagoner to the Department of Ecology, "Completion of Negotiations of Hanfor Federal Facility Agreement and Consent Order Milestones M-50-00, M-51-00, and M-60-00," dated December 21, 1995.

12: What assumptions were used in estimating the minimum and maximum waste percentages identified in Table 1?

For example, the minimum waste $\%$ is based upon the majority of the waste being sent to a commercial disposal site.

The maximum waste $\%$ is based upon additional projects being initiated that will generate waste not accounted for in the best estimate volume. The waste numbers are very preliminary and were generally given a range of $50 \%$ to $200 \%$, primarily due to the uncertainty of dealing with preliminary facility and processing concepts.

13: Please provide a description of how the radionuclides were determined?

For example, historical data or sample analysis data were used.

The radionuclide estimates are preliminary based on expected feed materials and processes. Reference WHC-SD-WM-TI-694, "Tank Waste

Remediation System Optimized Processing Strategy." The dominate radionuclides in the tanks are cesium-137 and strontium-90.

$14 \mathrm{a} / \mathrm{b} / \mathrm{c}$ : Indicate confidence levels for the following waste characterization data:

or example, the physical waste forms are based upon historical characterization information. 
Date: $6 / 20 / 96$

Waste Generator

Question Answer

Explain:

(c) Radiation Type

High

Low

Medium

Explain:

a. Meduium. The physical waste forms are based on waste historically generated by Hanford processing lacilities and the knowledge of the characteristics of tank waste.

b. Medium

c. Medium

WHC_LLVP

WHC_LLVP

3
1
8
$15 \mathrm{a} / \mathrm{b} / \mathrm{c}$ : (a) What percentage of the waste forecasted is existing waste and what percentage is future generated waste?

(b) Of your existing waste, how confident are you in the current shipping schedule? (High, Low, Medium) Explain:

(c) Of your future generated waste, how confident are you in the volumes and shipping schedule? (High, Low, Medium) Explain:

a. All waste is to be generated in the future.

b. N/A

c. Low, schedules are subject to change due to contracting uncertainties at Hanford and with the privatized contracts.

Does this forecast include:

(a) Operational waste?

(b) Terminal clean-out \& stabilization waste (TC\&S)?

(c) Decontamination \& Decommissioning waste (D\&D)?

a. Yes

b. No 


\begin{tabular}{cc} 
Waste Generator & $\begin{array}{c}\text { Question } \\
\text { Answer }\end{array}$ \\
\hline & $-\quad$ c. No
\end{tabular}

WHC_LLVP

17: If you have not included TC\&S or D\&D waste, will this waste potentially be managed at Hanford? (Yes/No)

If 'Yes', please provide an estimate of the expected volumes.

Terminal cleanout and D\&D will be after 2011 for the demonstration facility and after 2021 for the full scale facility. How this waste is handled is unknown.

WHC_LLVP 18: When will your facility transition into a terminal clean-out and stabilization mission? This is unknown.

WHC_LLVP $\quad$ 19: When is your facility expected to be added to the Surplus Facility List? This is unknown.

WHC_LLVP

20: Will waste be generated between terminal clean-out/stabilization and D\&D? (Yes/No)

If 'Yes', please provide a waste description and identify the organization and department that has responsibility for this ciose-out waste? This is unknown.

WHC_LLVP

21: What document titles and numbers can be provided that give insight into solid waste generation at your facility?

Please provide a list of document titles and document numbers that provide insight into solid waste generated during operations, terminal clean-out and stabilization activities and or D\&D activities. Reference WHC-SD-WM-TI-694, "Tank Waste Remediation System Optimized Processing Strategy."

WHC_LLVP

22: Does your facility have an approved Site Treatment Plan (STP) or a Proposed Site Treatment Plan (PSTP) in progress?

NOTE - OFFSITE GENERATORS ONLY, re: Richland Operations Office Letter (RL) \#95-SWT-174, Guidance for Acceptance of Offsite Mixed Waste. Not applicable

\begin{tabular}{ll}
\hline WHC_SST_LE & 1: Please indicate which program is \\
& EM-60 (Landlord) \\
& EM-40 (Environmental Restoration) \\
& EM-30... \\
Analytical Services \\
Liquid Waste \\
PNNL \\
RCRA Monitoring \\
Solid Waste \\
Spent Nuclear Fuels \\
Transition Projects \\
TWRS TWRS
\end{tabular}




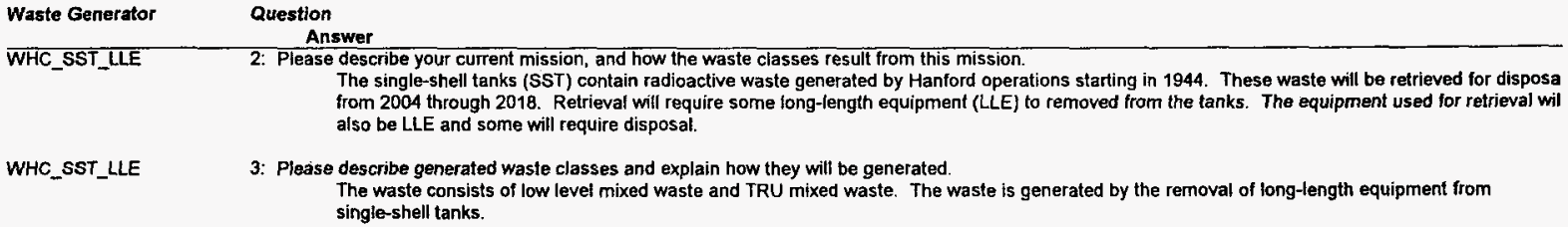

The single-shell tanks (SST) contain radioactive waste generated by Hanford operations starting in 1944. These waste will be retrieved for disposa from 2004 through 2018. Retrieval will require some long-length equipment (LLE) to removed from the tanks. The equipment used for retrieval wil also be LLE and some will require disposal.

WHC_SST_LLE

3: Piease describe generated waste classes and explain how they will be generated.

The waste consists of low level mixed waste and TRU mixed waste. The waste is generated by the removal of long-length equipment from single-shell tanks.

WHC SST_LLE

WHC_SST_LEE

WHC_SST_ULE
4: Please provide a description of your HELD waste i.e., waste forms.

Are the volumes for HELD waste different from last year's forecast? (Yes/No)

If Yes, Please explain.

Some of the existing equipment in tanks will require removal.

5: Have your waste volumes changed from your previous forecast? (Yes/No).

If 'Yes', please explain.

The waste volumes for LLE have changed based on a new estimate of the amount of existing equipment and the amount of equipment used for retrieval. A new analysis was done for the amount of waste which will be TRU. In addition, an agreement was reached with DOE tor the eventual disposal of the LLE. All equipment removed from the tanks after 2003 will be handled according to the new agreement.

6 a/b: (a) Have you excluded any waste generation from potential future projects that are not in the official baseline?

(b) Do you foresee any facilities that are not currentty your responsibility that will be in the future that are not forecasted?

(c)Are these variations provided in your extimation of the Min and Max values?

Please explain.
a. No
b. No
c. N/A

7: Will waste be separated into like waste forms prior to shipping?

For example, if you generate contaminated soils, inorganic particulates and metal debris, will you ship these waste in separate containers, or will the waste b combined and placed into one container?

Please explain for each waste class.

Each piece of equipment generally will be packaged individually. Each package will be handled according to its waste class.

WHC SST LLE

If 'Yes', (b) Will you place several hazardous constituents in one container or will you segregate the constituents prior to shipping? 


\section{Waste Generato}

WHC_SST_LLE

WHC_SST_LLE

WHC_SST_LLE

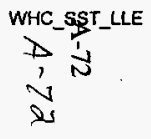

WHC_SST_LLE
Question

Questionnaire

Answer

That is, if you generate toxic metals and ignitables, will you ship these constituents in separate containers or will they be combined and placed into one container?

a. All of the waste removed from the taniks is considered mixed waste. Each piece of equipment is expected to be packaged individually. The contamination will be attached to the equipment.

b. Each piece of equipment is expected to be packaged individualiy.

9: Please describe your quarterly expected shipping schedule for each applicable waste class during FY 1997

For example: $40 \%$ of waste shipped during the first quarter, $20 \%$ shipped in the second quarter, $40 \%$ shipped in the fourth quarter. No waste is expected in 1997

10: Discuss the assumptions that were used to prepare this forecast.

The forecast is based on the worst case removal of equipment from SST. The removal of existing equipment occurs about three years before retrieval is scheduled to begin based on the latest retrieval sequence (Penwell, personal communication).

Retrieval is expected to use an average of two pumps and two sluicers per tank. One half of this equipment is included in the forecast. Disposal o this equipment occurs during the retrieval years. The other equipment is disposed in the same manner as the tanks and is not included in the forecast.

11: Please list any Tri-Party Agreement (TPA) milestones that affect your facility/program.

For each TPA milestone please indicate your status in achieving the milestone and whether these milestones are reflected in your forecast.

The related milestones are discussed in a memorandum from John Wagoner to the Department of Ecology, "Completion of Negotiations of Hanfor Federal Facility Agreement and Consent Order Milestones M-50-00, M-51-00, and M-60-00," dated December 21, 1995.

12. What assumptions were used in estimating the minimum and maximum waste percentages identified in Table 1?

For example, the minimum waste $\%$ is based upon the majority of the waste being sent to a commercial disposal site.

The maximum waste $\%$ is based upon additional projects being initiated that will generate waste not accounted for in the best estimate volume. The waste numbers are very preliminary and were generally given a range of $50 \%$ to $200 \%$, primarily due to the uncertainty of dealing with preliminary facility and processing cencepts.

13: Please provide a description of how the radionuclides were determined?

For example, historical data or sample analysis data were used.

The radionuclide estimates are preliminary based on expected feed materials and processes. Reference WHC-SD-WM-TI-694, "Tank Waste Remediation System Optimized Processing Strategy."

$14 \mathrm{a} / \mathrm{b} / \mathrm{c}$ : Indicate confidence levels for the following waste characterization data:

For example, the physical waste forms are based upon historical characterization information.

(a) Physical Waste Forms 


\section{Waste Generator Question}

\begin{tabular}{c} 
Waste Generator $\quad \begin{array}{c}\text { Question } \\
\text { Answer }\end{array}$ \\
\hline
\end{tabular}

High

Low

Medium

Explain:

(b) Hazardous Constituents

High

Low

Medium

Explain:

(c). Radiation Type

High

Low

Medium

Explain:

a. Medium. Equipment and tank waste characteristics are generally known within a factor of two and ten, respectively.

b. Medium

c. Medium

$15 \mathrm{a} / \mathrm{b} / \mathrm{c}$ : (a) What percentage of the waste forecasted is existing waste and what percentage is future generated waste?

(b) Of your existing waste, how confident are you in the current shipping schedule? (High, Low, Medium) Explain:

(c) Of your future generated waste, how confident are you in the volumes and shipping schedule? (High, Low, Medium)

Explain:

a. Most of the long-length equipment is existing equipment in the tanks. However, a significant percentage will be future equipment used for tank waste retrieval.

b. Medium. The shipping schedule depends on continuation of existing program plans and on budget allocations. 
Date: $6 / 20 / 96$

Waste Generator

WHC_SST_LLE

WHC_SST_LLE

WHC_SST_LLE

WHC_SST_LLE

.

WHC_SST_LLE

WHC_SST_LLE

$\frac{1}{1}$
SOLID WASTE FORECAST FY1997

Page:

Question

Answer

c. Medium. The shipping volumes are accurate, somewhat conservatively high. The shipping schedule will depend on continuation of the existing program plans and budgets.

$16 \mathrm{a} / \mathrm{b} / \mathrm{c}$ : Indicate whether your forecast includes: operational, terminal clean-out \& stabilization (TC\&S), and/or D\&D wastes.

Does this forecast include:

(a) Operational waste?

(b) Terminal clean-out \& stabilization waste (TC\&S)?

(c) Decontamination \& Decommissioning waste (D\&D)?

a. Yes. Only operational wastes are included. Tank closure and D\&D are not inciuded.

b. No.

c. No.

17: If you have not included TC\&S or D\&D waste, will this waste potentially be managed at Hanford? (Yes/No)

If 'Yes', please provide an estimate of the expected volumes. Unknown.

18: When will your facility transition into a terminal clean-out and stabilization mission? Unknown.

19: When is your facility expected to be added to the Surplus Facility List? Unknown.

20: Will waste be generated between terminal clean-out/stabilization and D\&D? (Yes/No)

If 'Yes'. please provide a waste description and identify the organization and department that has responsibility for this close-out waste? Unknown.

21: What document titles and numbers can be provided that give insight into solid waste generation at your facility?

Please provide a list of document ttles and document numbers that provide insight into solid waste generated during operations, terminal clean-out and stabilization activities and or D\&D activities.

Reference WHC-SD-WM-TI-694, "Tank Wasfe Remediation System Optimized Processing Strategy."

Letter, Jackson Kinzer, DOE-Richland to Westinghouse Hanford Company, dated April 11, 1996, "Tank Waste Remediation System (TWRS) Remote Handled Transuranic Waste Volume Forecast." 


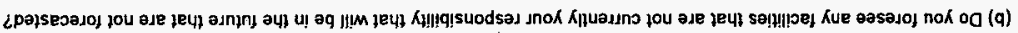

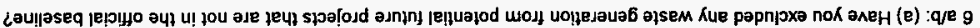

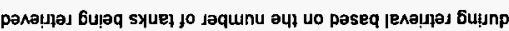

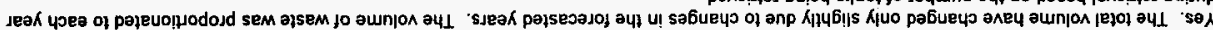

Hiejdxe eseajd ', sel,

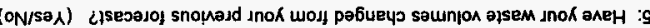

aUON

Ui!ejdxe eseerd 'sex II

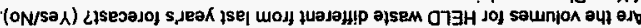

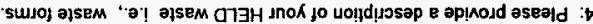

$1 \exists \mathbf{y}^{-1} 1 \mathrm{SS}^{-} \mathrm{OHM}$

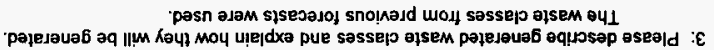

$1 \exists \mathrm{Y}^{-} \perp \mathrm{SS}^{-} \mathrm{\top} \mathrm{HM}$

8102 466nod4l tooz woy

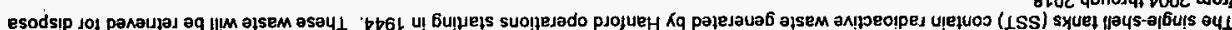

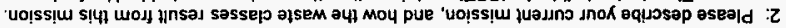

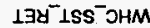

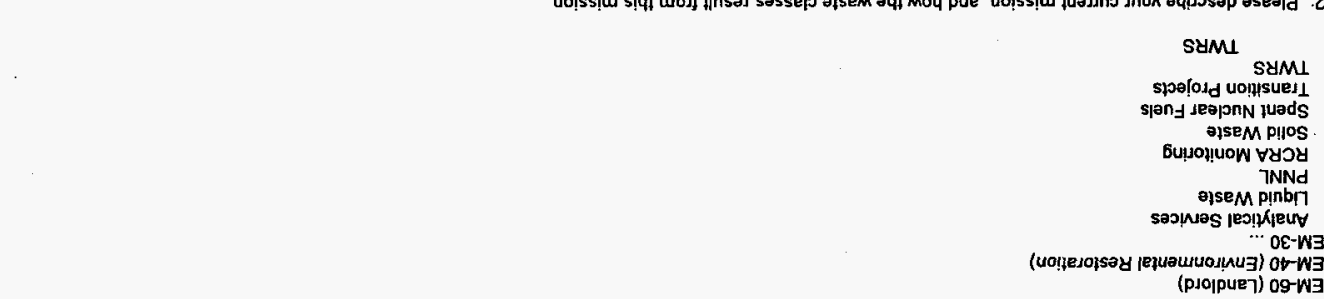

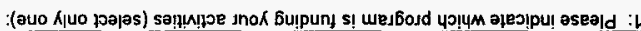

$134^{-1} 1 S^{-} \mathrm{HM}$

elqeo!!dde joN

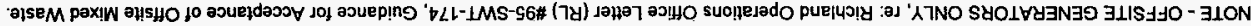

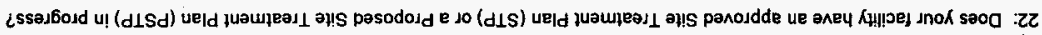

$\exists 77^{-1 S S^{-} \mathrm{OHM}}$

Jamsut

uopjsano

JopEJauag ө|sEM

aม!̣euuonsano

bt :abed

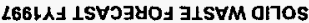

96/0Z/9 :əyea 


\section{Waste Generator Question}

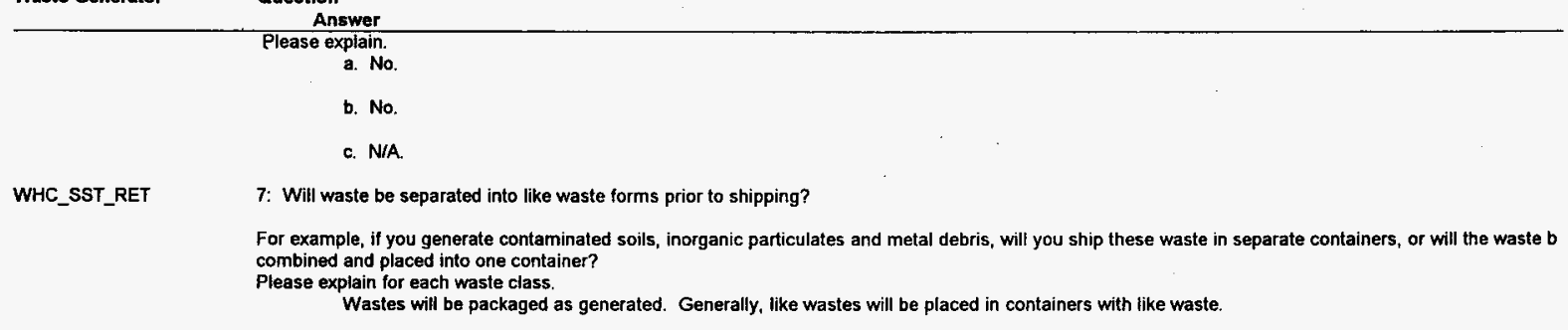

$8 \mathrm{a} / \mathrm{b}$ : (a) Will you generate hazardous or mixed waste? (Yes/No)

If 'Yes', (b) Will you place several hazardous constituents in one container or will you segregate the constituents prior to shipping?

That is, if you generate toxic metals and ignitables, will you ship these constituents in separate containers or will they be combined and placed into one container?

a. Yes. The tanks contain mixed waste and a majority of the solid waste will be mixed waste.

b. Hazardous materials will be segregated to the extent possible.

WHC_SST_RET

9: Please describe your quarterly expected shipping schedule for each applicable waste class during FY 1997.

For example: $40 \%$ of waste shipped during the first quarter, $20 \%$ shipped in the second quarter, $40 \%$ shipped in the fourth quarter. None.

WHC_SST_RET

WHC_SST_RET

1)

WHC_SST_RET

10: Discuss the assumptions that were used to prepare this forecast.

The total volume from the previous forecast was used.

11: Please list any Tri-Party Agreement (TPA) milestones that affect your facility/program.

For each TPA milestone please indicate your status in achieving the milestone and whether these milestones are reflected in your forecast. The related milestones are discussed in a memorandum from John Wagoner to the Department of Ecology, "Completion of Negotiations of Henfor Federal Facility Agreement and Consent Order Milestones M-50-00, M-51-00, and M-60-00," dated December 21, 1995.

12: What assumptions were used in estimating the minimum and maximum waste percentages identified in Table 1 ?

For example, the minimum waste $\%$ is based upon the majority of the waste being sent to a commercial disposal site.

The maximum waste $\%$ is based upon additional projects being initiated that will generate waste not accounted for in the best estimate volume.

The waste numbers are very preliminary and were generally given a range of $50 \%$ to $200 \%$, primarily due to the uncertainty of dealing with preliminary facility and processing concepts. 
Date: $6 / 20 / 96$

$:$ :

SOLID WASTE FORECAST FY1997

Questionnaire

Waste Generato

Question

Answer

WHC_SST_RET

13: Please provide a description of how the radionuclides were determined?

For example, historical data or sample analysis data were used.

The radionuclide estimates are preliminary based on expected feed materials and processes. Reference WHC-SD-WM-TI-694, Tank Waste

Remediation System Optimized Processing Strategy." The radionuclides in the tank waste are dominated by cesium- 137 and strontium- 90 .

WHC_SST_RET

$14 \mathrm{a} / \mathrm{b} / \mathrm{c}$ : Indicate confidence levels for the following waste characterization data:

For example, the physical waste forms are based upon historical characterization information.

(a) Physical Waste Forms

High

Low

Medium

Explain:

(b) Hazardous Constituents

High

Low

Medium

Explain:

(c) Radiation Type

High

Low

Medium

Explain:

a. Medium. Retrieval operations are ill-defined at this time

b. Medium. Retrieval operations are ill-defined at this time

c. Medlum. Retrieval operations are ill-defined at this time. 


\section{Waste Generator}

WHC_SST_RET

WHC_SST_RET
Question

Answer

$15 \mathrm{a} / \mathrm{b} / \mathrm{c}$ : (a) What percentage of the waste forecasted is existing waste and what percentage is future generated waste?

(b) Of your existing waste, how confident are you in the current shipping schedule? (High, Low, Medium)

Explain:

(c) Of your future generated waste, how confident are you in the volumes and shipping schedule? (High, Low, Medium)

Explain

a. The waste is future generated waste produced by retrieval operations

b. N/A

c. Medium. Retrieval operations are it-defined and the schedule is subject to change

$16 \mathrm{a} / \mathrm{b} / \mathrm{c}$ : Indicate whether your forecast includes: operational, terminal clean-out \& stabilization (TC\&S) ,and/or D\&D wastes

Does this forecast include:

(a) Operational waste?

(b) Terminal clean-out \& stabilization waste (TC\&S)?

(c) Decontamination \& Decommissioning waste (D\&D)?

a. Yes

b. No

c. No

17: If you have not included TC\&S or D\&D waste, will this waste potentially be managed at Hanford? (Yes/No)

If 'Yes', please provide an estimate of the expected volumes. Unknown.

18: When will your facility transition into a terminal clean-out and stabilization mission? Unknown.

19: When is your facility expected to be added to the Surplus Facility List? Unknown.

20: Will waste be generated between terminal clean-out/stabilization and D\&D? (Yes/No)

If 'Yes', please provide a waste description and identify the organization and department that has responsibility for this close-out waste? 
Date: $6 / 20 / 96$

SOLID WASTE FORECAST FY1997

Page:

Waste Generator Question

Questionnaire

Answer

WHC_SST_RET 21: What document titles and numbers can be provided that give insight into solid waste generation at your facility?

Please provide a list of document titles and document numbers that provide insight into solid waste generated during operations, terminal clean-out and stabilization activities and or D\&D activities.

Reference WHC-SD-WM-TI-694, "Tank Waste Remediation System Optimized Processing Strategy."

WHC-EP-0900, "FY1996 Solid Waste Integrated Life-Cycle Forecast Volume Summary," February, 1996.

WHC_SST_RET

22: Does your facility have an approved Site Treatment Plan (STP) or a Proposed Sile Treatment Plan (PSTP) in progress?

NOTE - OFFSITE GENERATORS ONLY, re: Richland Operations Office Letter (RL) \#95-SWT-174, Guidance for Acceptance of Offsite Mixed Waste. Not applicable 
Date: $6 / 19 / 96$

SOLID WASTE FORECAST FY1997

Notes

Waste Generator Waste Class

Container

Physical Waste Form

HC or RAD

Potential demonstration start in 2002 (Phase 1). Full scale operation will begin about 2013 and end about 2028.

WHC_LIV

Phase 1 demonstration of low level vitrification begins in 2002. Phase 1 may continue through 2011. Phase 2 operations run from 2011 through 2021.

$5 / 21 / 96$ No

WHC_SST_LLE

All SST retrieval completed by 2028 .

George Reddick

$5 / 21 / 96$ No

WHC SST RET

Complete demonstration on one tank by 2003. Complete all SST retrieval by 2018 . 
Abbreviations

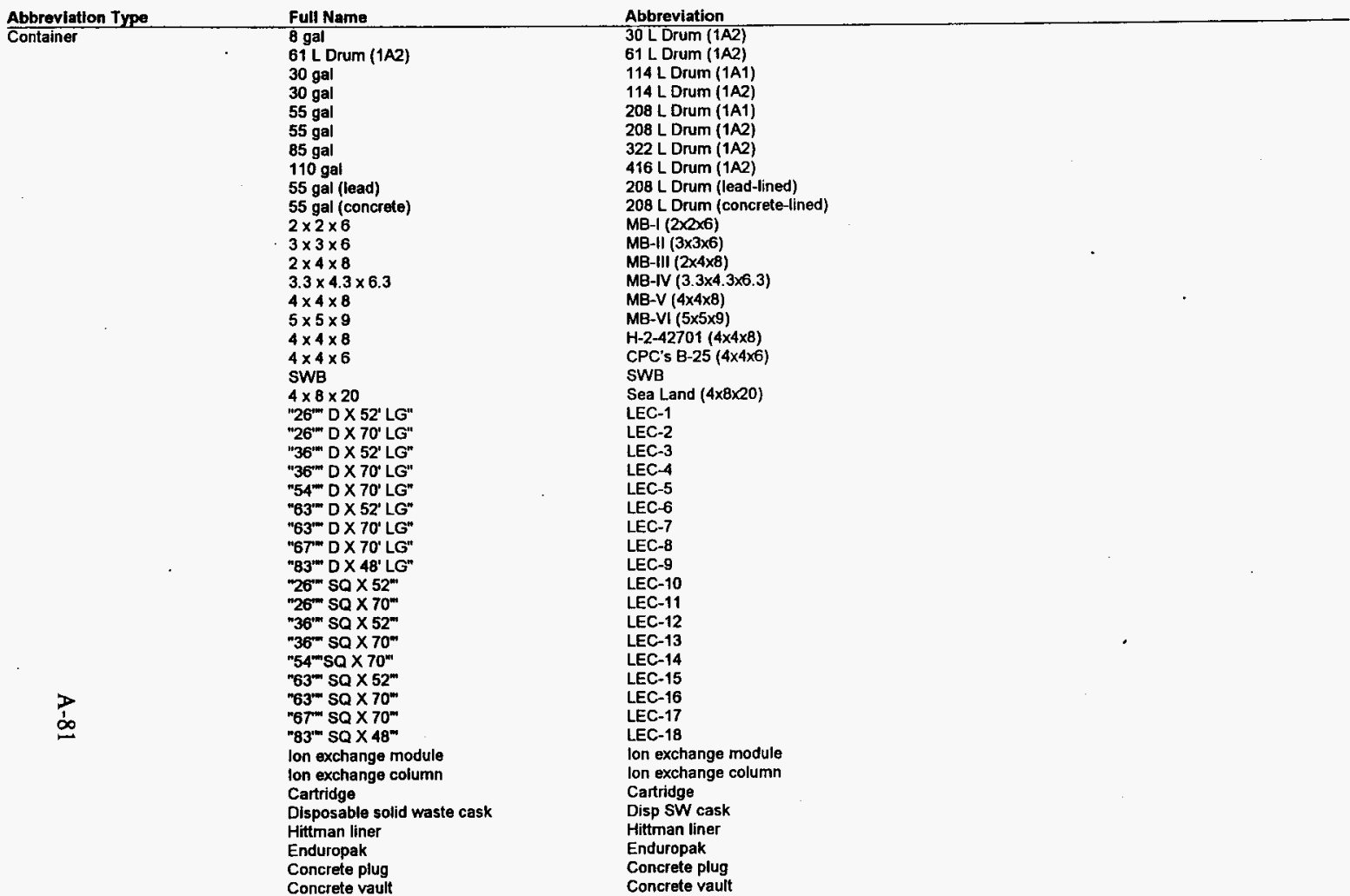




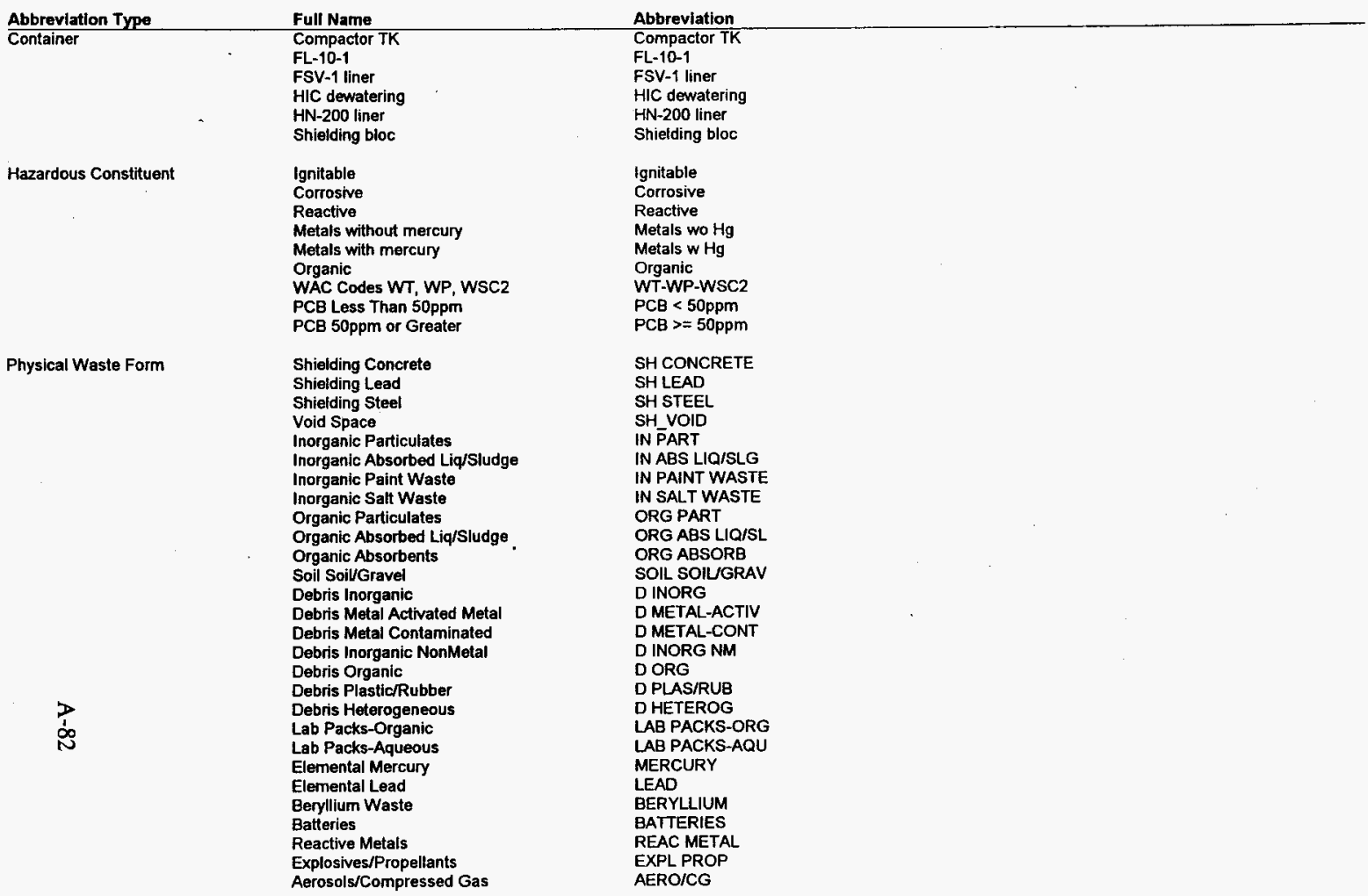

SORB

INORG

LAB PACKS-ORG

BERYLLIUM

REAC METAL 
Abbrevlation Type

Full Name

Radion uclide

CARBON14

Radium 22

Technetium 99

Selenium 79

Plutonium 238, 239,240

Plutonium 241

Neptunium 237

Uranium 233

Uranium 234

Uranium 235

Uranium 238

lodine 129

lodiine 131

Tritium

Americium 241,243

Chlorine 36

Cesium 137

Strontium 90

Phosphorus 32

Sulfur 35

Coball 60

Lead 210

RADIUM226

TECHNETIUM99

SELENIUM79

PLUTONIUM

PLUTONIUM241

NEPTUNIUM237

URANIUM233

URANIUM234

URANIUM235

URANIUM238

IODINE129

IODINE131

TRITIUM

AMERICIUM

CHLORINE36

CESIUM137

STRONTIUM9O

PHOSPHOROUS32

SULFUR35

COBALT6O

LEAD210 
Date: $6 / 19 / 96$

SOLID WASTE FORECAST FY1997

Complete Data Summary

Page: 1

Waste Generator

Waste Class, Contalner, Physical Waste Form, (Radionuclide or Hazardous Constituent)

$\frac{\text { WHC HLVP }}{\text { CHLLW. }}$

\begin{tabular}{|c|c|c|c|c|c|c|c|c|c|c|c|c|c|c|c|}
\hline $\begin{array}{l}\text { Vol } \\
\text { Minx } \\
\text { Maxx }\end{array}$ & Held & 1997 & 1998 & 1999 & 2000 & 2001 & $\begin{array}{r}2002 \\
50 \\
50 \\
200\end{array}$ & $\begin{array}{r}2003 \\
50 \\
50 \\
200\end{array}$ & $\begin{array}{r}2004 \\
50 \\
50 \\
200\end{array}$ & $\begin{array}{r}2005 \\
50 \\
50 \\
200\end{array}$ & $\begin{array}{r}2006 \\
50 \\
50 \\
200\end{array}$ & $\begin{array}{r}2007 \\
50 \\
50 \\
200\end{array}$ & $\begin{array}{r}2008 \\
50 \\
50 \\
200\end{array}$ & 2009 & 2010 \\
\hline $\begin{array}{l}\text { Vol } \\
\text { Mint } \\
\text { Mex* }\end{array}$ & 2011 & 2012 & $\begin{array}{r}2013 \\
250 \\
50 \\
200\end{array}$ & $\begin{array}{r}2014 \\
250 \\
50 \\
200\end{array}$ & $\begin{array}{r}2015 \\
250 \\
50 \\
200\end{array}$ & $\begin{array}{r}2016 \\
250 \\
50 \\
200\end{array}$ & $\begin{array}{r}2017 \\
250 \\
50 \\
200\end{array}$ & $\begin{array}{r}2018 \\
250 \\
50 \\
200\end{array}$ & $\begin{array}{r}2019 \\
250 \\
50 \\
200\end{array}$ & $\begin{array}{r}2020 \\
250 \\
50 \\
200\end{array}$ & $\begin{array}{r}2021 \\
250 \\
50 \\
200\end{array}$ & $\begin{array}{r}2022 \\
250 \\
50 \\
200\end{array}$ & $\begin{array}{r}2023 \\
250 \\
50 \\
200\end{array}$ & $\begin{array}{r}2024 \\
250 \\
50 \\
200\end{array}$ & $\begin{array}{r}2025 \\
250 \\
50 \\
200\end{array}$ \\
\hline
\end{tabular}

\begin{tabular}{lrrr} 
& 2026 & 2027 & 2028 \\
Vol & 250 & 250 & 250 \\
Minx & 50 & 50 & 50 \\
Max* & 200 & 200 & 200 \\
\multicolumn{2}{c}{ End of Ufecycle Reached } \\
M
\end{tabular}

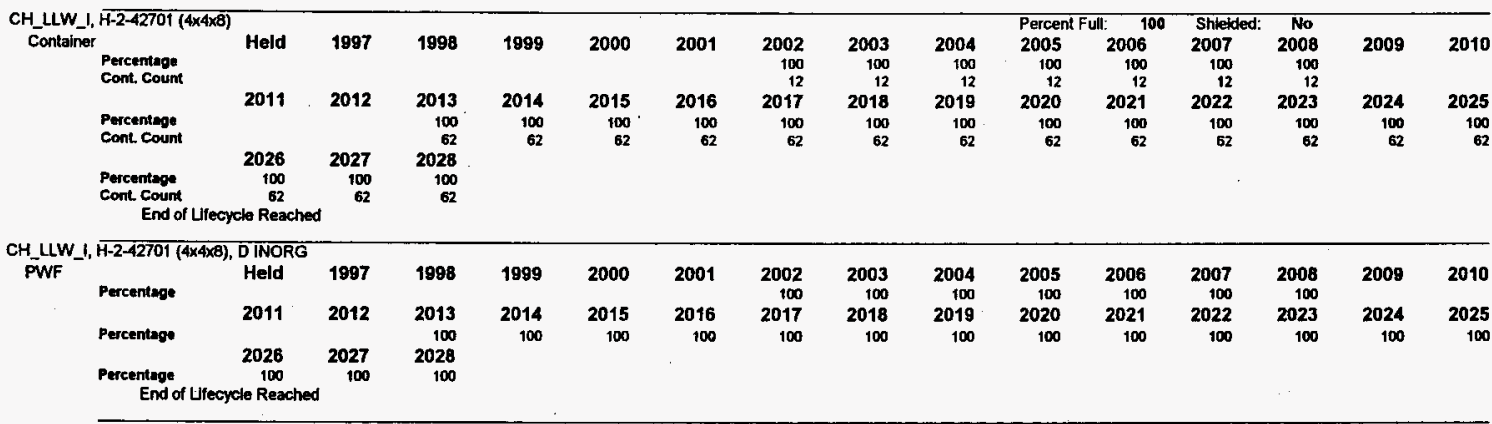

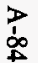


Waste Generator

Waste Class, Container, Physical Waste Form, (Radionuclide or Hazardous Constituent)

CH_LLW_I, H-2-42701 (4x4×8), D INORG, CESIUM137

RAD

concentration.

Held $1997^{1} 1998$

Concentration

2011

20122013

1999. 2000

20012002

2003

\section{4}

2005

$\begin{array}{rrrrrr}005 & 2006 & 2007 & 2008 & 2009 & 2010 \\ .001 & .001 & .001 & .001 & & \\ 001 & 2021 & 2022 & 2023 & 2024 & 2025 \\ .001 & .001 & .001 & .001 & .001 & .001\end{array}$

Concentration $\quad$\begin{tabular}{rrr}
2026 & 2027 & 2028 \\
\hline & 001 & .001
\end{tabular}

End of Liecycle Reached

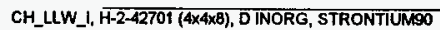

RAD

\begin{tabular}{lrrr} 
Concentration & Heid & 1997 & 1 \\
& 2011 & 2012 & 2013 \\
Concentration & & & \\
& 2026 & 2027 & 2028 \\
Concentration & $\begin{array}{c}.001 \\
\text { End of Uifecycle Reached }\end{array}$ & .001 & \\
\hline
\end{tabular}

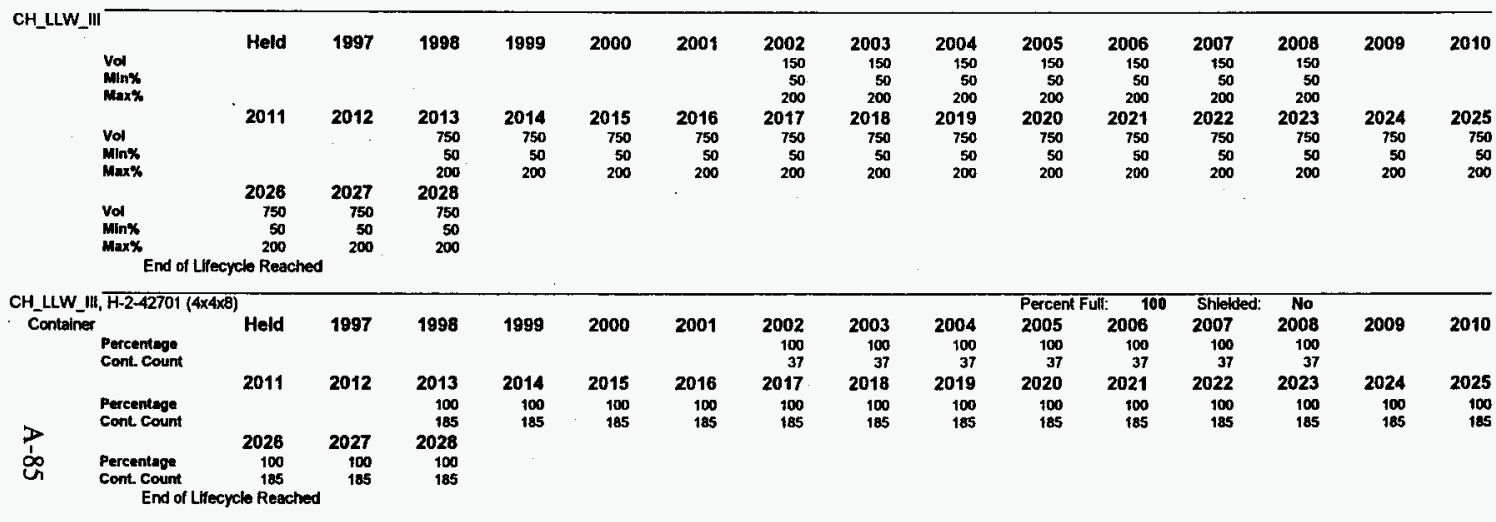


Waste Generator

Waste Class, Container, Physical Waste Form, (Radionuclide or Hazardous Constituent)

PWF

$\begin{array}{llll}\text { Percentsge } & \text { Held } & 1997 & 1998 \\ \text { Percentuge } & 2011 & 2012 & 2013 \\ & 2026 & 2027 & 2028 \\ \text { Percentsge } & \begin{array}{l}100 \\ \text { End of Lifecycte Reached }\end{array} & 100 & \\ \end{array}$

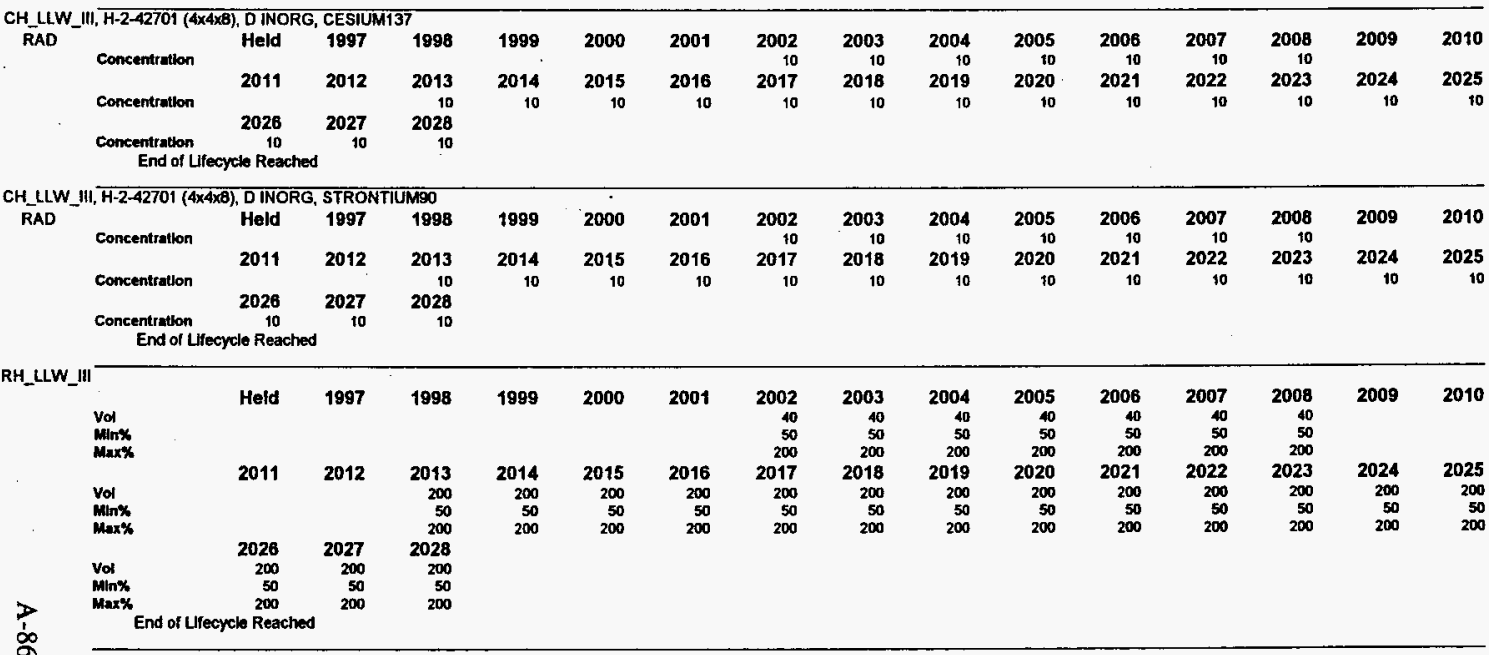


Waste Generator

Waste Class, Container, Physical Waste Form, (Radionuclide or Hazardous Constituent)

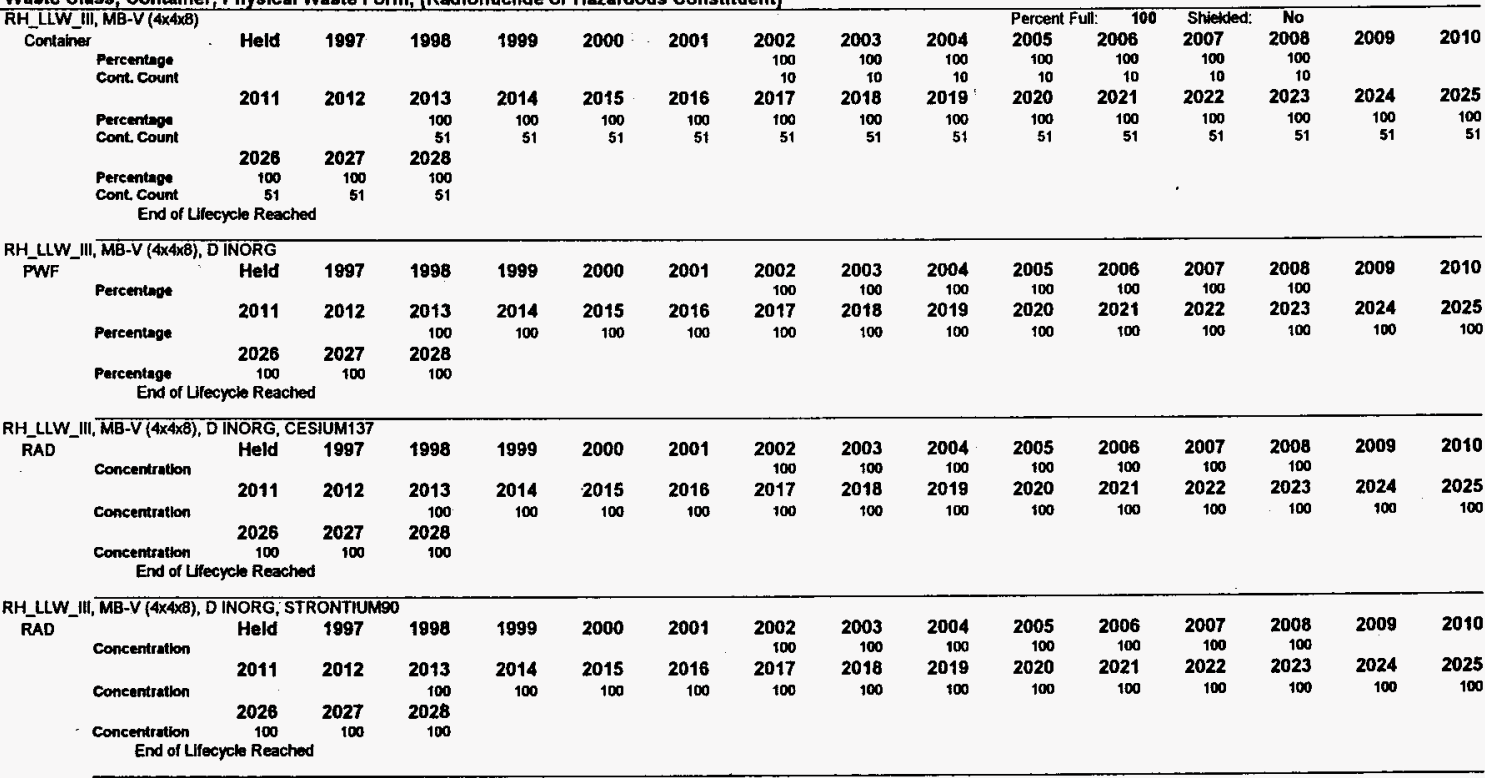

$\underset{⿱ 亠 乂}{\dot{j}}$ 
Waste Generator

Waste Class, Container, Physical Waste Form, (Radlonuclide or Hazardous Constituent)

CH_LLMW_I

\begin{tabular}{|c|c|c|c|c|c|c|c|c|c|c|c|c|c|c|c|}
\hline $\begin{array}{l}\text { Vol } \\
\text { Min\% } \\
\text { Max* }\end{array}$ & Held & 1997 & 1998 & 1999 & 2000 & 2001 & $\begin{array}{r}2002 \\
20 \\
50 \\
200\end{array}$ & $\begin{array}{r}2003 \\
20 \\
50 \\
200\end{array}$ & $\begin{array}{r}2004 \\
20 \\
50 \\
200\end{array}$ & $\begin{array}{r}2005 \\
20 \\
50 \\
200\end{array}$ & $\begin{array}{r}2006 \\
20 \\
50 \\
200\end{array}$ & $\begin{array}{r}2007 \\
20 \\
50 \\
200\end{array}$ & $\begin{array}{r}2008 \\
20 \\
50 \\
200\end{array}$ & 2009 & 2010 \\
\hline ink & 2011 & 2012 & $\begin{array}{r}2013 \\
t 00 \\
50 \\
200\end{array}$ & $\begin{array}{r}2014 \\
100 \\
50 \\
200\end{array}$ & $\begin{array}{r}2015 \\
100 \\
50 \\
200\end{array}$ & $\begin{array}{r}2016 \\
100 \\
50 \\
200\end{array}$ & $\begin{array}{r}2017 \\
100 \\
50 \\
200\end{array}$ & $\begin{array}{r}2018 \\
100 \\
50 \\
200\end{array}$ & $\begin{array}{r}2019 \\
100 \\
50 \\
200\end{array}$ & $\begin{array}{r}2020 \\
100 \\
50 \\
200\end{array}$ & $\begin{array}{r}2021 \\
100 \\
50 \\
200\end{array}$ & $\begin{array}{r}2022 \\
100 \\
50 \\
200\end{array}$ & $\begin{array}{r}2023 \\
100 \\
50 \\
200\end{array}$ & $\begin{array}{r}2024 \\
100 \\
50 \\
200\end{array}$ & $\begin{array}{r}2025 \\
100 \\
50 \\
200\end{array}$ \\
\hline
\end{tabular}

$\begin{array}{lrrr} & 2026 & 2027 & 2028 \\ \text { Vol } & 100 & 100 & 100 \\ \text { Mln\% } & 50 & 50 & 50\end{array}$

$\begin{array}{crrr}\text { Min\% } & 50 & 50 & 100 \\ \text { Max\% } & 200 & 50 \\ \text { End of Lifecycle Reached } & 200 & 200\end{array}$

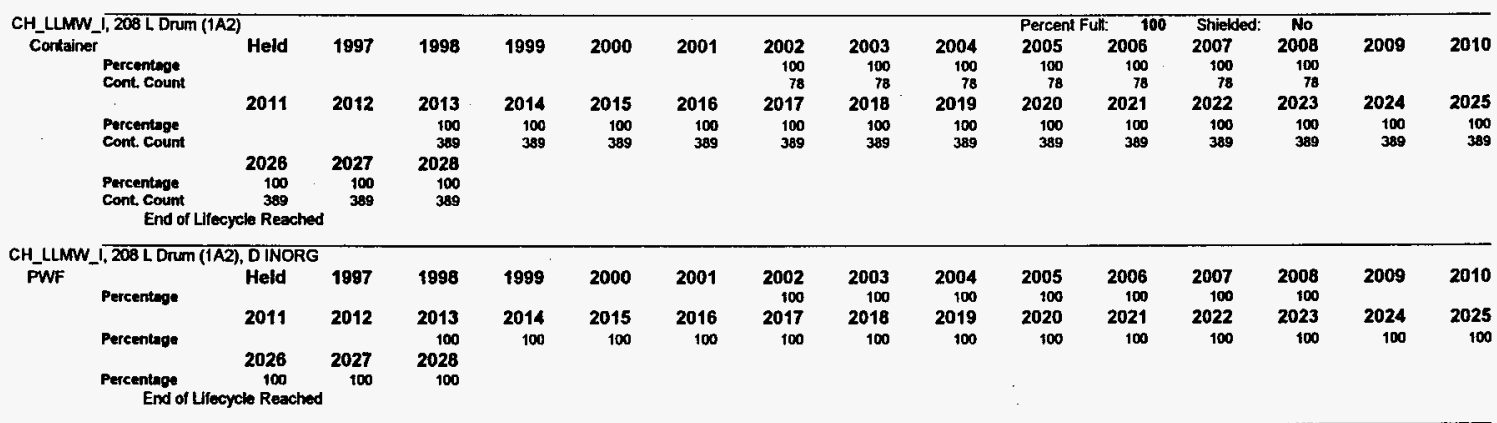

$\underset{\infty}{\infty}$ 
Waste Generator

Waste Class, Container, Physical Waste Form, (Radionuclide or Hazardous Constituent)

CH_LLMW_, 208 L Drum (1A2), D INORG, WT-WP WSC2

$\mathrm{HC}$

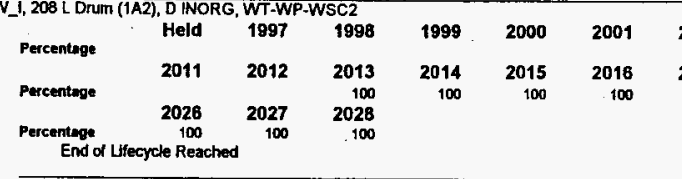

\begin{tabular}{|c|c|c|c|c|c|c|c|c|c|c|c|c|c|c|c|c|}
\hline \multirow{5}{*}{$\underset{\text { RAD }}{\mathrm{CH}_{-} \text {UW }}$} & 1,208 L Drum & ), DINO & CESIU & & & & & & & & & & & & & \\
\hline & Concentration & Held & 1997 & 1998 & 1999 & 2000 & 2001 & $\begin{array}{r}2002 \\
.001\end{array}$ & $\begin{array}{r}2003 \\
.001\end{array}$ & $\begin{array}{r}2004 \\
.001\end{array}$ & $\begin{array}{r}2005 \\
.001\end{array}$ & $\begin{array}{r}2006 \\
.004\end{array}$ & $\begin{array}{r}2007 \\
.001\end{array}$ & $\begin{array}{r}2008 \\
.001\end{array}$ & 2009 & 2010 \\
\hline & & 2011 & 2012 & 2013 & 2014 & 2015 & 2016 & 2017 & 2018 & 2019 & 2020 & 2021 & 2022 & 2023 & 2024 & 2025 \\
\hline & Concentration & & & 001 & .001 & .001 & .001 & .001 & .001 & .001 & .001 & .001 & .001 & .001 & .001 & .001 \\
\hline & $\begin{array}{l}\text { Concentration } \\
\text { End of } L\end{array}$ & $\begin{array}{r}2026 \\
.001 \\
\text { cle Rea }\end{array}$ & $\begin{array}{r}2027 \\
.001\end{array}$ & $\begin{array}{r}2028 \\
.001\end{array}$ & & & & & & & & & & & & \\
\hline
\end{tabular}

CH_LLMW_, 208 LDRUM (1A2), D INORG, STRONNTIUNCO

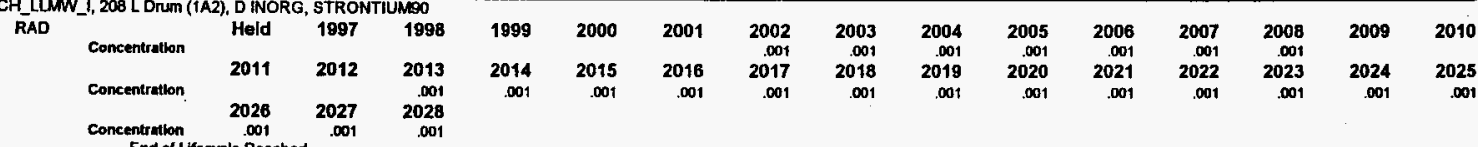

End of Lifecycle Reached

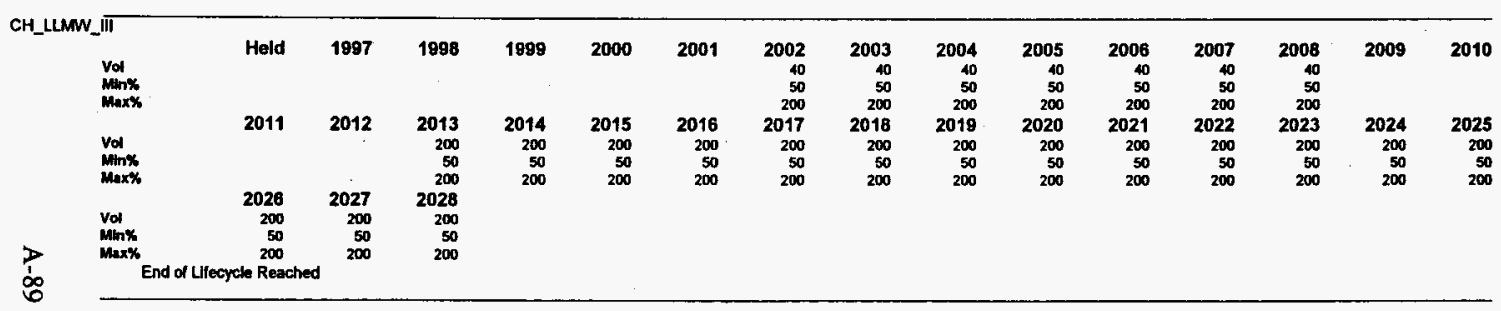


Waste Generator

Waste Class; Container; Physical Waste Form, (Radionuclide or Hazardous Constituent)

CH_LLMW_III, 208 L Drum (1A2)

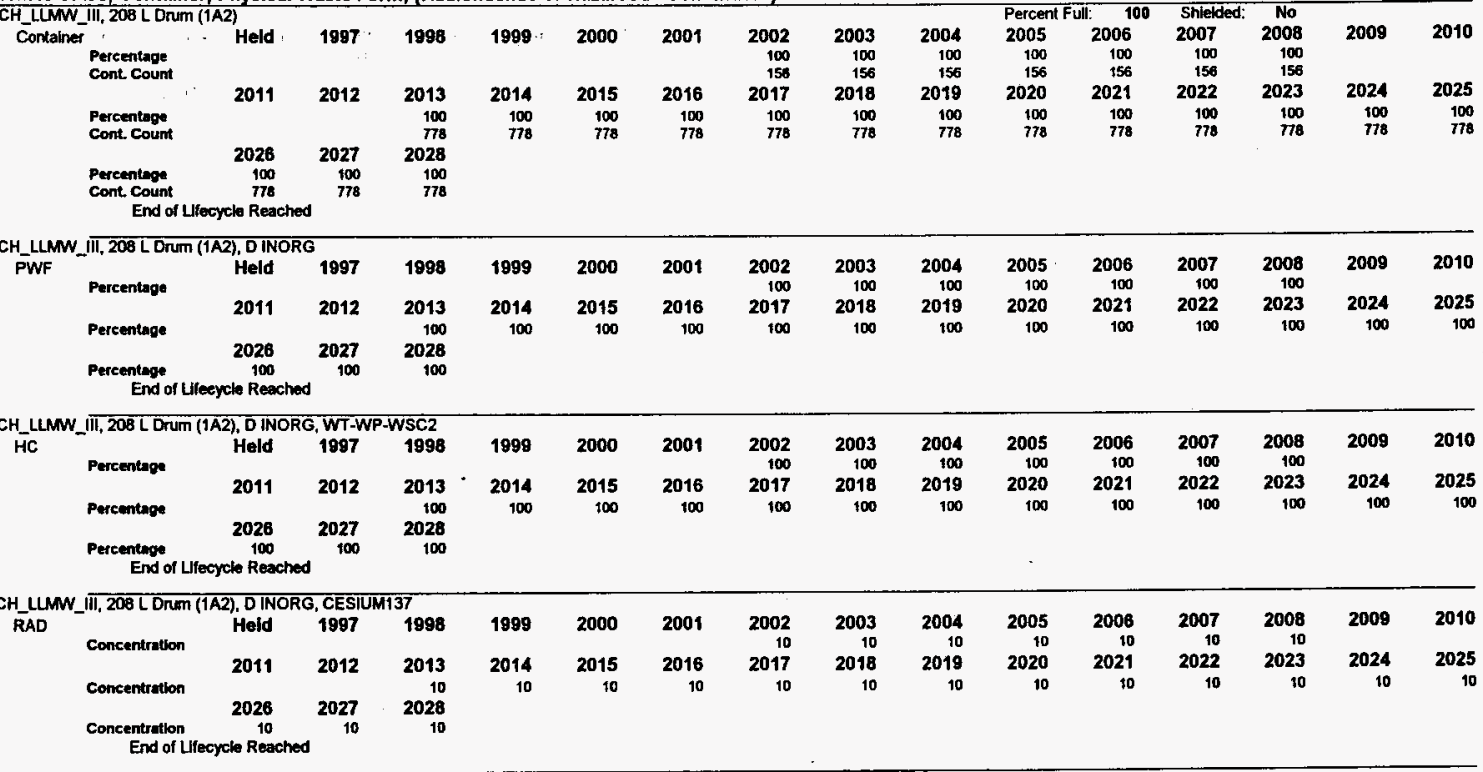

安 
Waste Generator

Waste Class, Contalner, Physical Waste Form, (Radionuclide or Hazardous Constituent)

CH_LLMW_III, 208 L D DUm (1A2), DINORG, STRONTIUMMO

RAD

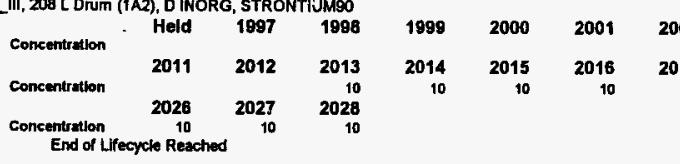

$\begin{array}{rr}2003 & 2004 \\ 2018 & 2019\end{array}$

00042005

100

$\begin{array}{rrrr}2007 & 2008 & 2009 & 2010 \\ 10 & 10 & & \\ 2022 & 2023 & 2024 & 2025 \\ 10 & 10 & 10 & 10\end{array}$

CH_TRUM End of Lifecycle Reached

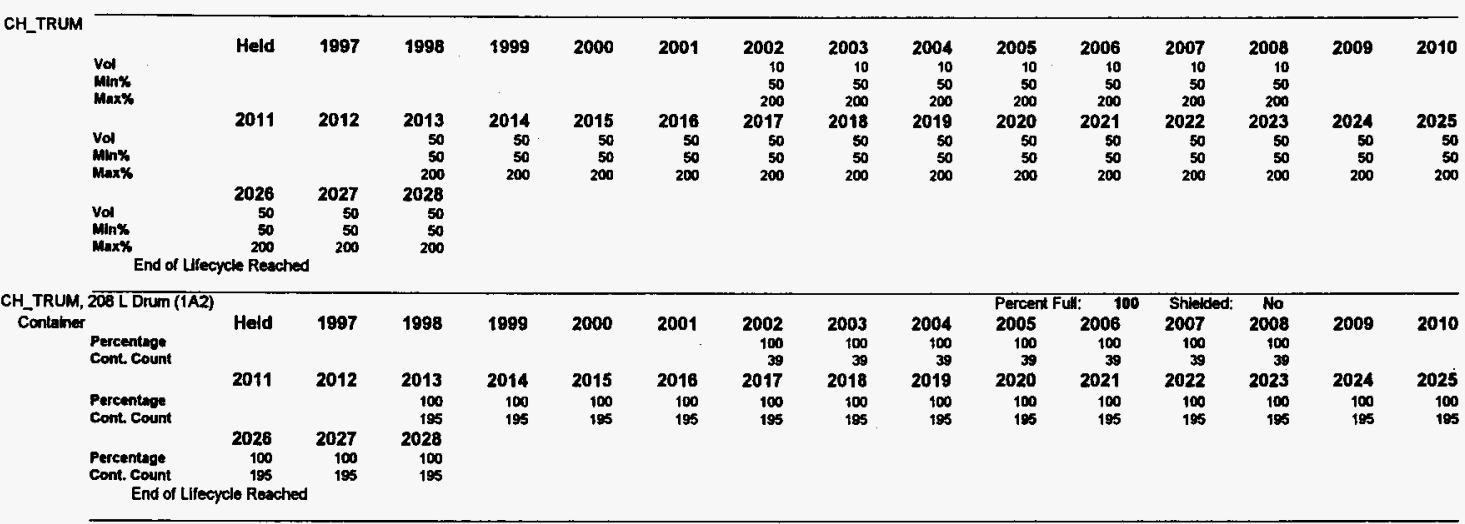

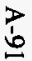


Waste Generator

Waste Class, Container, Physical Waste Form, (Radlonuclide or Hazardous Constituent)

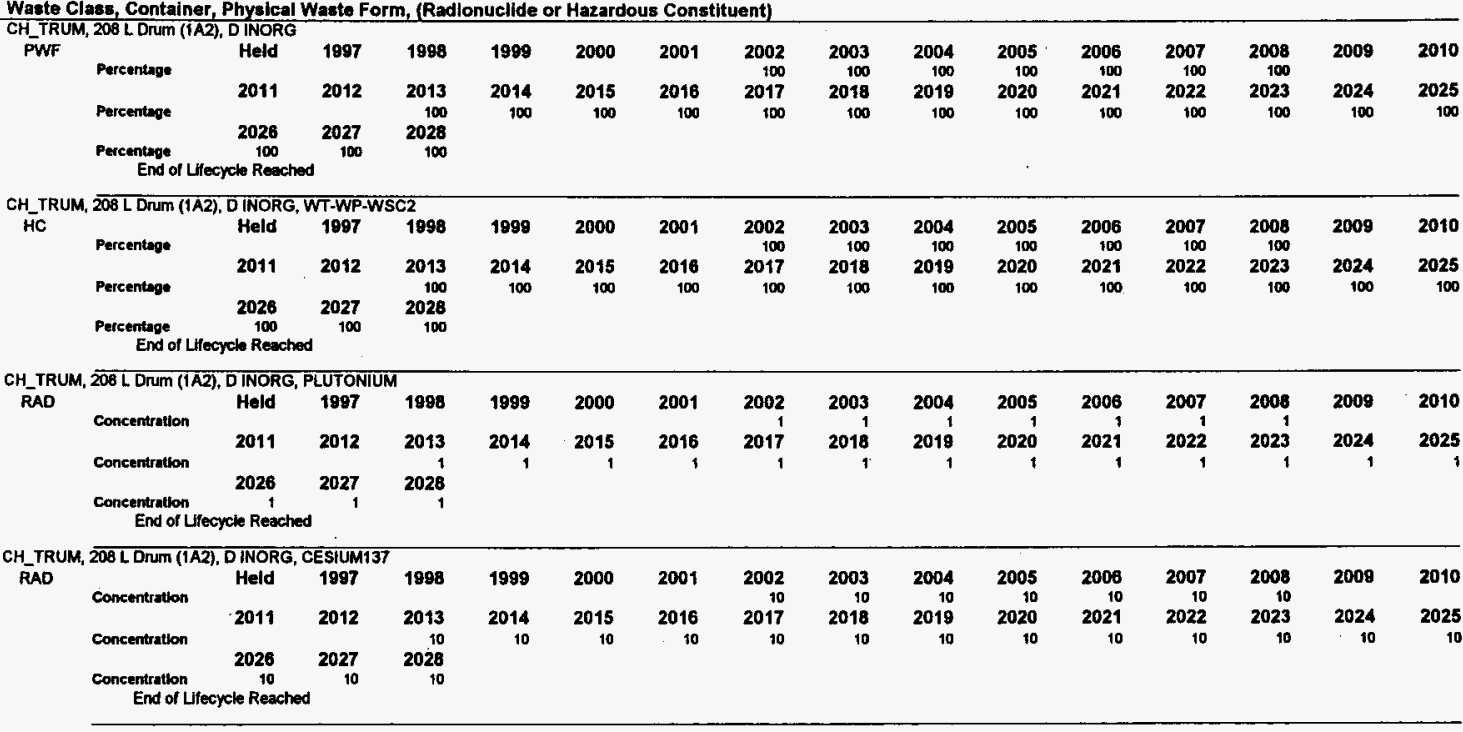


Waste Generator

Waste Class, Container, Physical Waste Form, (Radionuclide or Hazardous Constituent

CH_tRUM, 208 L Drum (1A2), D INORG, STRONTIÜMO

$$
\text { RAD }
$$

$\begin{array}{lrrrrrrr}\text { Concentration } & \text { Held } & 1997 & 1998 & 1989 & 2000 & 2001 & 2002 \\ & 2011 & 2012 & 2013 & 2014 & 2015 & 2016 & 2017 \\ \text { Concentration } & 2026 & 2027 & 2028 & 10 & 10 & 10 & \\ \begin{array}{c}\text { Concentration } \\ \text { End of Lifecycie Reached }\end{array} & 10 & 10 & & & & \end{array}$

$\begin{array}{rrrrrrrrr}2002 & 2003 & 2004 & 2005 & 2006 & 2007 & 2008 & 2009 & 2010 \\ 10 & 10 & 10 & 10 & 10 & 10 & 10 & & \\ 2017 & 2018 & 2019 & 2020 & 2021 & 2022 & 2023 & 2024 & 2025 \\ 10 & 10 & 10 & 10 & 10 & 10 & 10 & 10 & 10\end{array}$

RH_TRUM

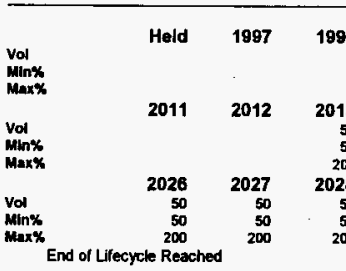

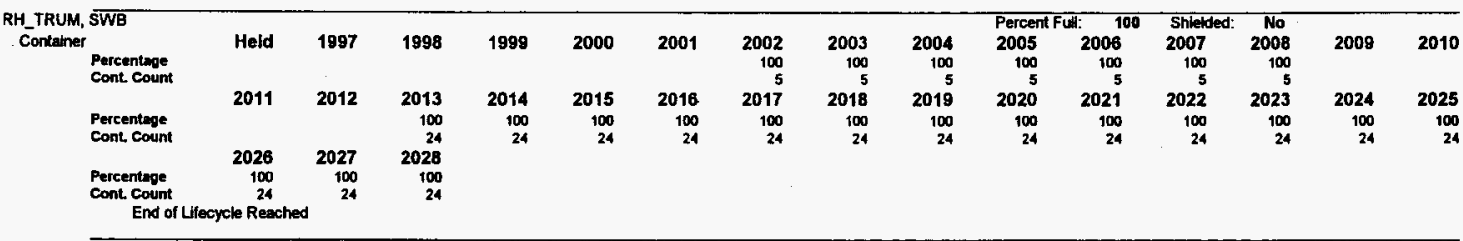


Waste Generator

Waste Class, Contalner, Physical Waste Form, (Radlonuclide or Hazardous Constituent

RH_TRUM, SWB, DINORG

PWF

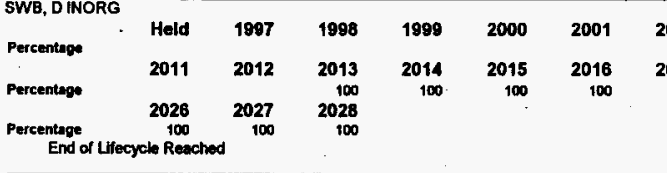

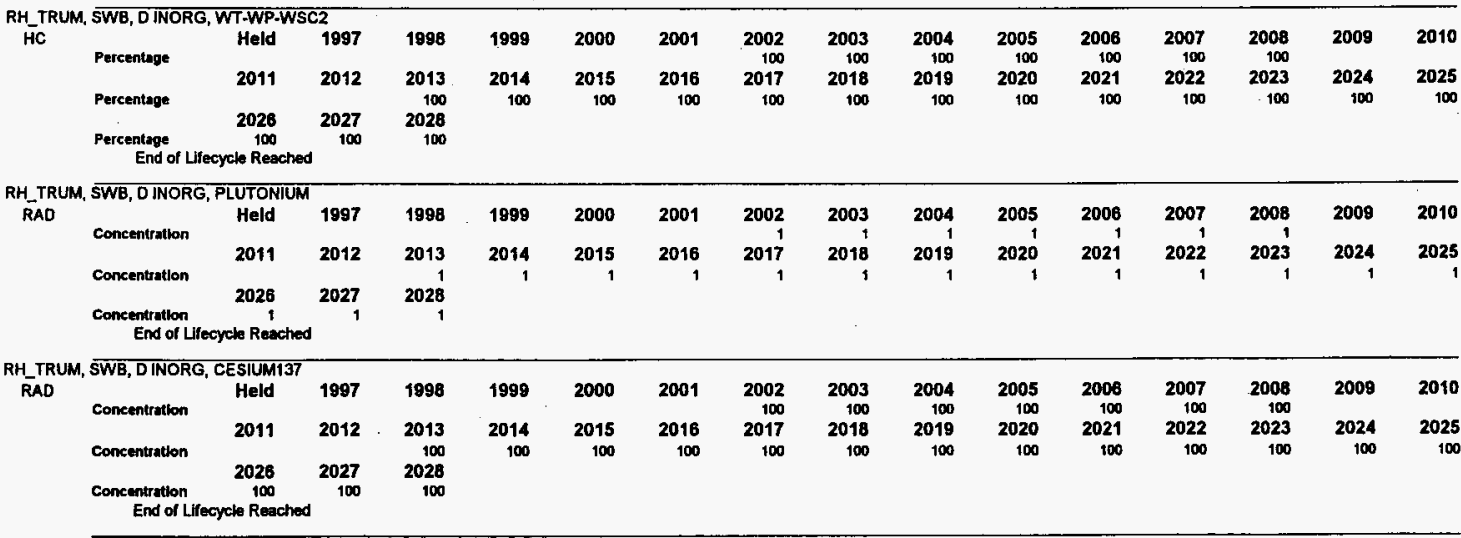

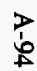


Waste Generator

Waste Claes, Container, Physical Waste Form, (Radlonucllde or Hazardous Constituent)

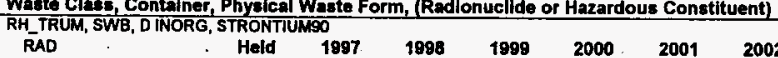

$\begin{array}{rrrrrrrr}\text { Concentration } & \text { Held } & 1997 & 1998 & 1998 & 2000 & 2001 & 2008 \\ & 2011 & 2012 & 2013 & 2014 & 2015 & 2016 & 2010 \\ \text { Concentration } & 2026 & 2027 & 2028 & 100 & 100 & 100 & \end{array}$

2003

2004

2005

$\begin{array}{rrrr} & 2026 & 2027 & 2028 \\ \text { Concentration } & 100 & 100 & 100\end{array}$
End of Ufecycle Reached

HAZ

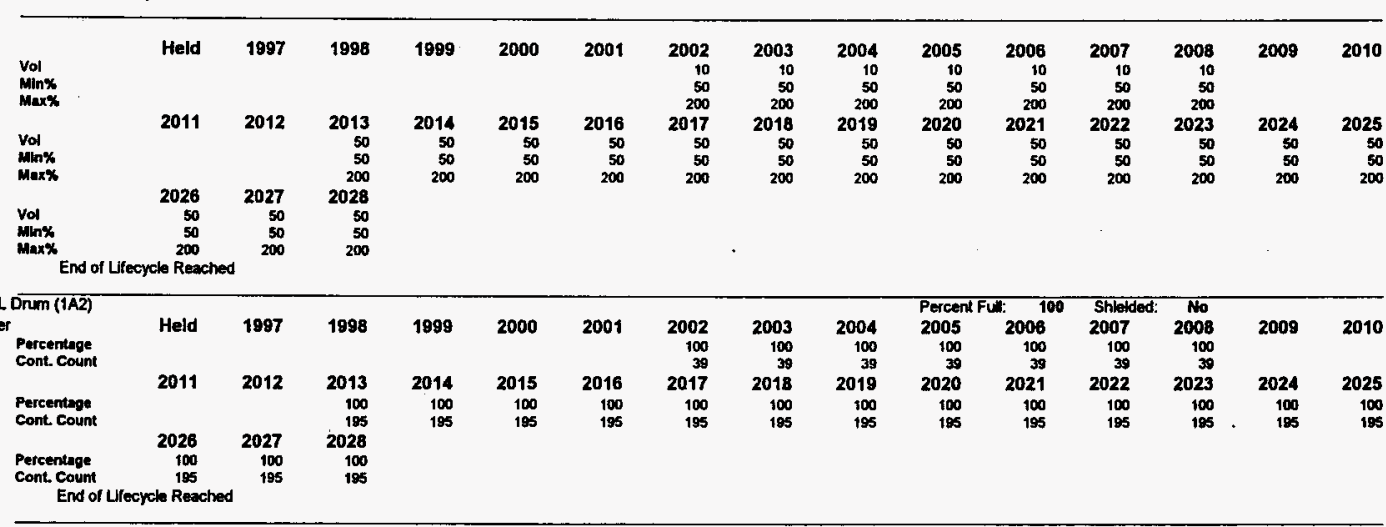

点 
Waste Generator

Waste Class Container, Physical Waste Form, (Radionuclide or Hazardous Constlituent)

HAZ 208 D Drum (1A2) DINORG

PWF

$\begin{array}{llll}\text { Percentage } & \text { Held } & 1997 & 199 \\ \text { Percentage } & 2011 & 2012 & 201 \\ & 2026 & 2027 & 202 \\ \text { Petcentage } & 100 & 100 & \\ \text { End of Llfecycle Reached } & \end{array}$

HAZ, 208 L DRUm (1A2), D INORG, WT-WP-WSC2
HC

$\begin{array}{lrrr}\text { Percentage } & \text { Held } & 1997 & 1 \\ & 2011 & 2012 & 20 \\ \text { Percentage } & & & \\ & 2026 & 2027 & 2028 \\ \text { Percentage } & 100 & 100 & \end{array}$

End of Lifecycle Reached

$\begin{array}{rrrr}1999 & 2000 & 2001 & 2002 \\ 2014 & 2015 & 2016 & 2017 \\ 100 & 100 & 100 & 100\end{array}$

$\begin{array}{rr}2002 & 2003 \\ 100 & 2017 \\ 100 & 2010\end{array}$

$\begin{array}{rr}2003 & 2004 \\ 100 & 100\end{array}$

2005
100
2020

2006

2007

2008

20092010

100

2019
100

2020
100

$\begin{array}{rrr}100 & 100 & 100\end{array}$

2021

100

2022
100

2023

2024 2025

$\begin{array}{rrrr}1999 & 2000 & 2001 & 2002 \\ 2014 & 2015 & 2016 & 2017\end{array}$

$\begin{array}{rrrr}2003 & 2004 & 2005 \\ 100 & 100 & 100 \\ 2018 & 2019 & 2020 & 202\end{array}$

$\begin{array}{rrrrr}2006 & 2007 & 2008 & 2009 & 2010 \\ 100 & 100 & 100 & & \\ 2021 & 2022 & 2023 & 2024 & 2025 \\ 100 & 100 & 100 & 100 & 100\end{array}$




\section{Waste Generator}

Waste Class, Container, Physical Waste Form, (Radionuclide or Hazardous Constituent) WHC LLVP

CH_LLW_I

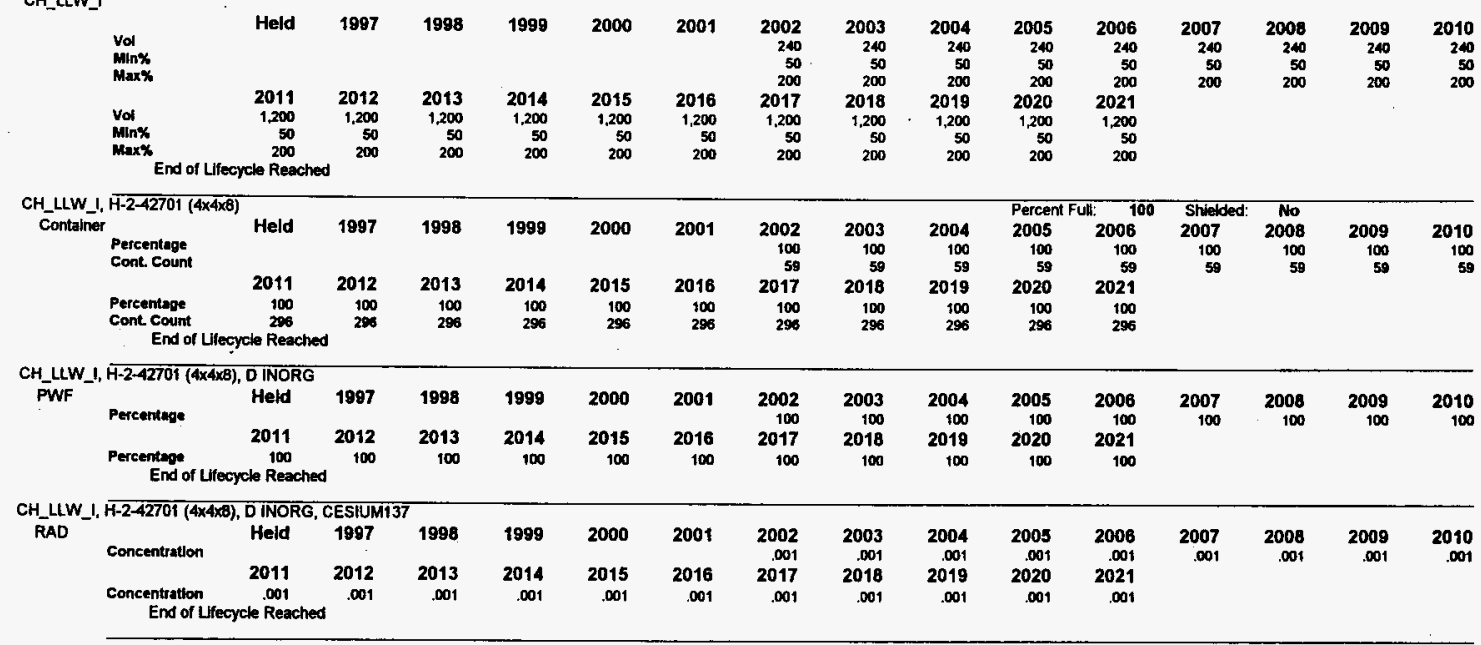

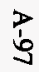


Waste Generator

Waste Class, Container, Physical Waste Form, (Radionuclide or Hazardous Constituent)

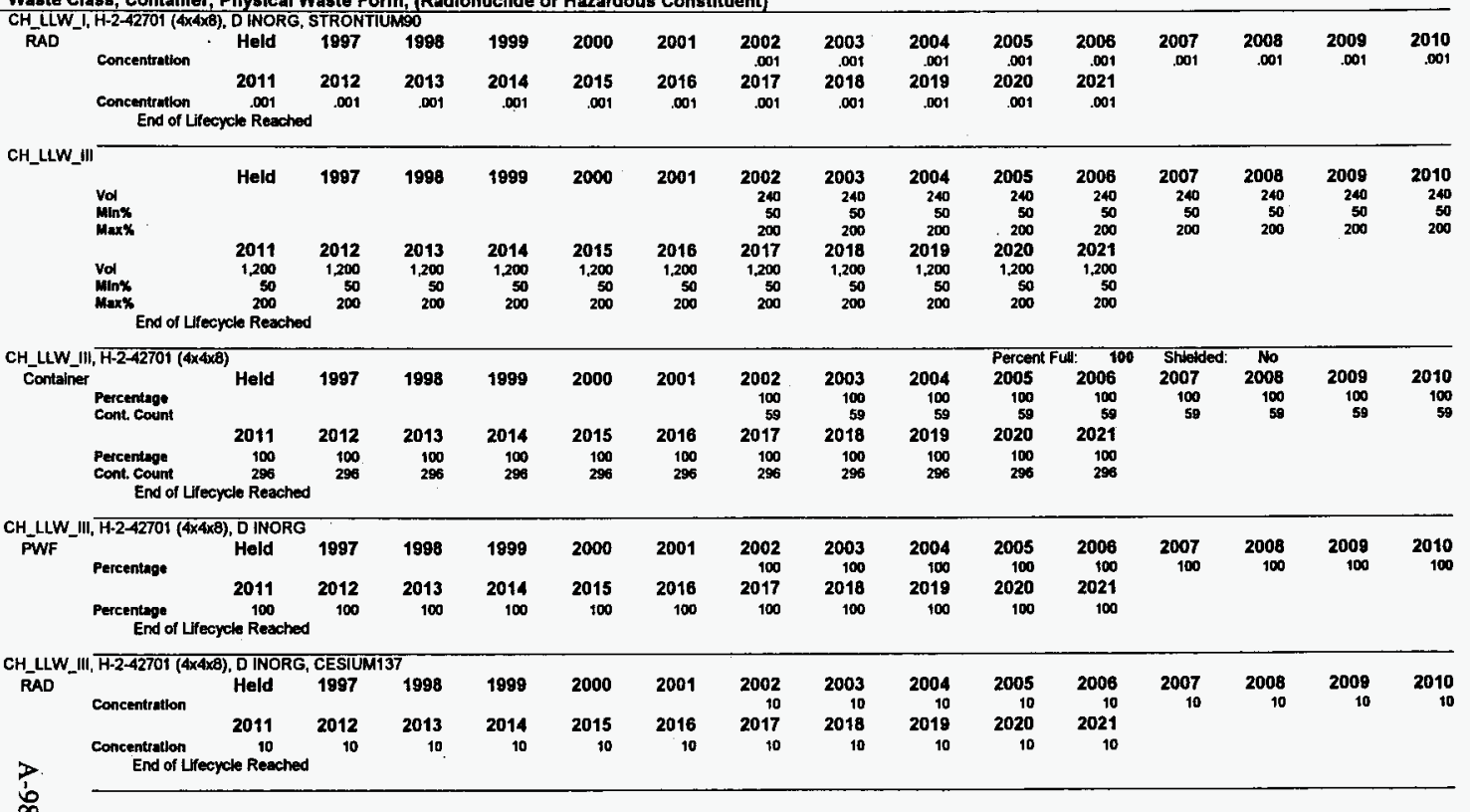


Waste Generator

Waste Class, Contalner, Physical Waste Form, (Radlonuclide or Hazardous Constituent)

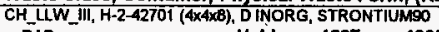

RAD

\begin{tabular}{lrrrrrrr} 
Concentration & Heid & 1997 & 1998 & 1999 & 2000 & 2001 & 2002 \\
& 2011 & 2012 & 2013 & 2014 & 2015 & 2016 & 2017 \\
$\begin{array}{c}\text { Concentration } \\
\text { End of Lifecycle Reached }\end{array}$ & 10 & 10 & 10 & 10 & 10 & \\
\hline
\end{tabular}

$\begin{array}{rr}2002 & 2003 \\ 10 & 10 \\ 2017 & 2018 \\ \text { to } & 10\end{array}$

2004
10
2019

2005
10

$\begin{array}{rr}10 & 2006 \\ 2020 & 2021\end{array}$

2021

\begin{tabular}{|c|c|c|c|c|c|c|c|c|c|c|c|c|c|c|c|c|}
\hline RH_LLW_III & $\begin{array}{l}\text { Nol } \\
\text { Min\% } \\
\text { Max* } \\
\text { Vol } \\
\text { Min\% } \\
\text { Max* End of Lif }\end{array}$ & $\begin{array}{r}2011 \\
300 \\
50 \\
200 \\
\text { yele Reac }\end{array}$ & $\begin{array}{r}2012 \\
300 \\
50 \\
200 \\
\end{array}$ & $\begin{array}{r}2013 \\
300 \\
50 \\
200\end{array}$ & $\begin{array}{r}2014 \\
300 \\
50 \\
200\end{array}$ & $\begin{array}{r}2015 \\
300 \\
50 \\
200\end{array}$ & $\begin{array}{r}2001 \\
\\
2016 \\
300 \\
50 \\
200\end{array}$ & $\begin{array}{r}2002 \\
60 \\
50 \\
200 \\
2017 \\
300 \\
50 \\
200\end{array}$ & $\begin{array}{r}2003 \\
60 \\
50 \\
200 \\
2018 \\
300 \\
50 \\
200\end{array}$ & $\begin{array}{r}2004 \\
60 \\
50 \\
200 \\
2019 \\
300 \\
50 \\
200\end{array}$ & $\begin{array}{r}2005 \\
60 \\
50 \\
200 \\
2020 \\
300 \\
50 \\
200\end{array}$ & $\begin{array}{r}2006 \\
60 \\
50 \\
200 \\
2021 \\
300 \\
50 \\
200\end{array}$ & $\begin{array}{r}2007 \\
60 \\
50 \\
200\end{array}$ & $\begin{array}{r}2008 \\
60 \\
50 \\
200\end{array}$ & $\begin{array}{r}2009 \\
60 \\
50 \\
200\end{array}$ & $\begin{array}{r}2010 \\
80 \\
50 \\
200\end{array}$ \\
\hline$\underset{\text { Container }}{R H}$ & $\begin{array}{l}\text { It, MB-V (4x4)(8) } \\
\text { Percentuge } \\
\text { Cont. Count } \\
\text { Percentuge } \\
\text { Cont. Count } \\
\text { End of Lite }\end{array}$ & $\begin{array}{r}\text { Held } \\
\\
2011 \\
100 \\
76 \\
\text { ycle Reac }\end{array}$ & $\begin{array}{r}1997 \\
2012 \\
100 \\
76\end{array}$ & $\begin{array}{r}1998 \\
2 \\
2013 \\
100 \\
76\end{array}$ & $\begin{array}{r}1999 \\
\\
2014 \\
100 \\
76\end{array}$ & $\begin{array}{r}2000 \\
\\
2015 \\
100 \\
76\end{array}$ & $\begin{array}{r}2001 \\
2016 \\
100 \\
76\end{array}$ & $\begin{array}{r}2002 \\
100 \\
15 \\
2017 \\
100 \\
76\end{array}$ & $\begin{array}{r}2003 \\
100 \\
15 \\
2018 \\
100 \\
76\end{array}$ & $\begin{array}{r}2004 \\
100 \\
15 \\
2019 \\
100 \\
76\end{array}$ & $\begin{array}{c}\text { Percent } \\
2005 \\
100 \\
15 \\
2020 \\
100 \\
76\end{array}$ & $\begin{array}{c}\text { dil: } 100 \\
2006 \\
100 \\
15 \\
2021 \\
100 \\
76\end{array}$ & $\begin{array}{l}\text { Shiclded: } \\
2007 \\
100 \\
15\end{array}$ & $\begin{array}{r}\text { No } \\
2008 \\
100 \\
15\end{array}$ & $\begin{array}{r}2000 \\
t 00 \\
15\end{array}$ & $\begin{array}{r}2010 \\
100 \\
15\end{array}$ \\
\hline 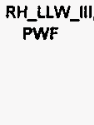 & $\begin{array}{l}\text { II, MB-V }(4 \times 4 \times 6) \\
\text { Percentage } \\
\text { Percentage } \\
\text { End of Life }\end{array}$ & $\begin{array}{l}\text { INORG } \\
\text { Held } \\
2011 \\
100 \\
\text { yele Reac }\end{array}$ & $\begin{array}{r}1997 \\
2012 \\
100\end{array}$ & $\begin{array}{r}1998 \\
2013 \\
100\end{array}$ & $\begin{array}{r}1999 \\
2014 \\
100\end{array}$ & $\begin{array}{r}2000 \\
2015 \\
100\end{array}$ & $\begin{array}{r}2001 \\
2016 \\
100\end{array}$ & $\begin{array}{r}2002 \\
100 \\
2017 \\
100\end{array}$ & $\begin{array}{r}2003 \\
100 \\
2018 \\
100\end{array}$ & $\begin{array}{r}2004 \\
100 \\
2019 \\
100\end{array}$ & $\begin{array}{r}2005 \\
100 \\
2020 \\
100\end{array}$ & $\begin{array}{r}2008 \\
100 \\
2021 \\
100\end{array}$ & $\begin{array}{r}2007 \\
100\end{array}$ & $\begin{array}{r}2008 \\
100\end{array}$ & $\begin{array}{r}2009 \\
100\end{array}$ & $\begin{array}{r}2010 \\
100\end{array}$ \\
\hline RH_LLW_III $_{\text {RAD }}$ & $\begin{array}{l}\text { MB-V }(4 \times 4 \times 8) \text {, } \\
\text { Concentration } \\
\text { Concentration } \\
\text { End of Life }\end{array}$ & $\begin{array}{l}\text { INORG, } \\
\text { Meld } \\
2011 \\
\text { to0 } \\
\text { cie Reac }\end{array}$ & $\begin{array}{r}\text { SIUM137 } \\
\begin{array}{r}1997 \\
2012 \\
100\end{array}\end{array}$ & $\begin{array}{r}1998 \\
2013 \\
100\end{array}$ & $\begin{array}{r}1999 \\
2014 \\
100\end{array}$ & $\begin{array}{r}2000 \\
2015 \\
100\end{array}$ & $\begin{array}{r}2001 \\
2016 \\
100\end{array}$ & $\begin{array}{r}2002 \\
100 \\
2017 \\
100\end{array}$ & $\begin{array}{r}2003 \\
100 \\
2018 \\
100\end{array}$ & $\begin{array}{r}2004 \\
100 \\
2019 \\
100\end{array}$ & $\begin{array}{r}2005 \\
100 \\
2020 \\
100\end{array}$ & $\begin{array}{r}2008 \\
100 \\
2021 \\
100\end{array}$ & $\begin{array}{r}2007 \\
100\end{array}$ & $\begin{array}{r}2008 \\
100\end{array}$ & $\begin{array}{r}2009 \\
100\end{array}$ & $\begin{array}{r}2010 \\
100\end{array}$ \\
\hline
\end{tabular}


Waste Generator

Waste Class, Container, Physical Waste Form, (Radionuclide or Hazardous Constituent)

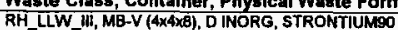

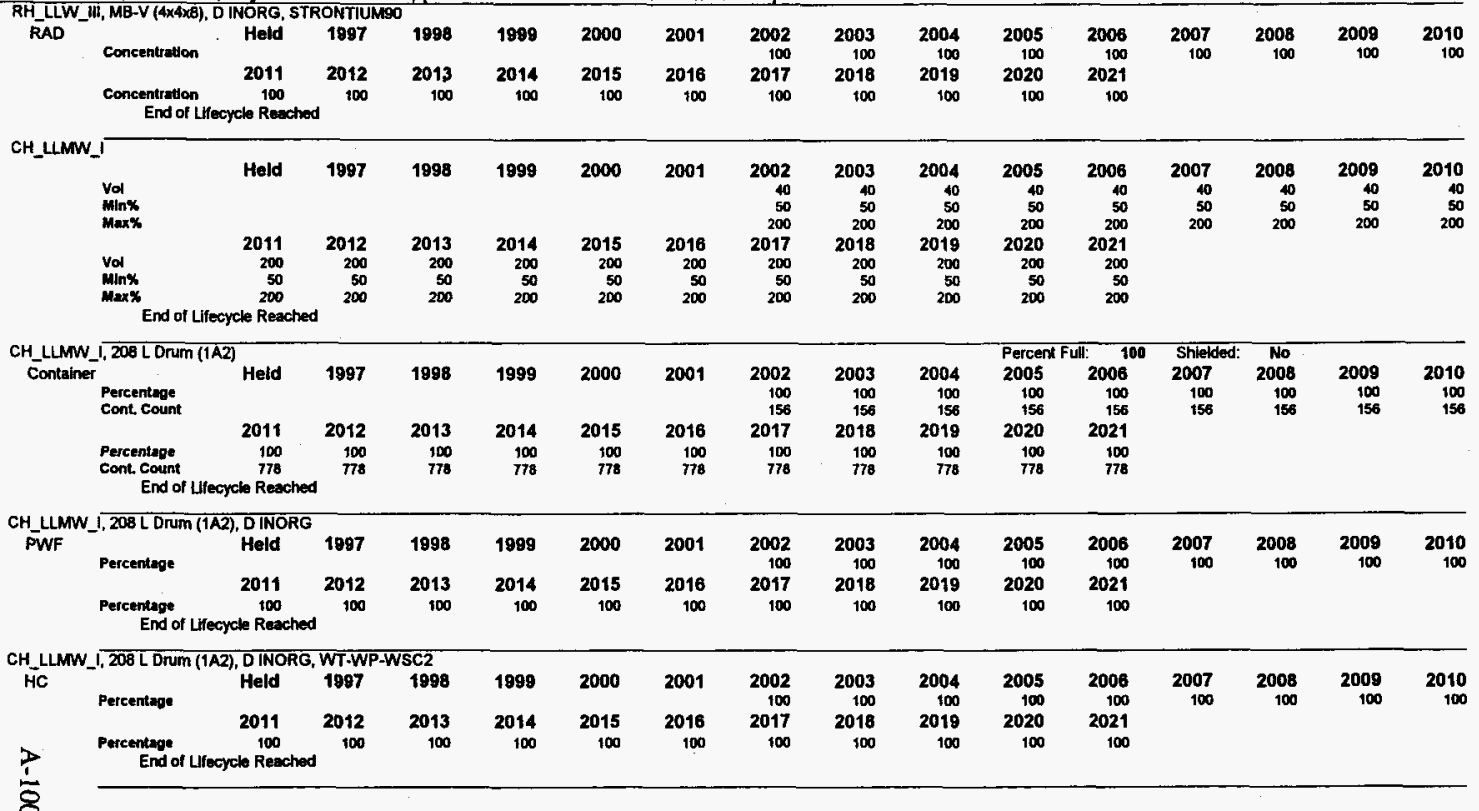


Waste Generator

Waste Class, Contelner, Physical Waste Form, (Radionuclide or Hazardous Constituent)

CH_LLMW_I, 208 L Drum (IAZ), DINORG, CESIUMI37

RAD

\begin{tabular}{lrrrrrr} 
& & & & \\
Concentration & Held & 1997 & 1998 & 1999 & 2000 & 2001 \\
Concentration & 2011 & 2012 & 2013 & 2014 & 2015 & 2016 \\
End of Lifecycle Reached & .001 & .001 & .001 & .001 & .001 \\
\hline
\end{tabular}

CH_LLMW_1, 208 L DIUM (1A2), D INORG, STRONTIUM9

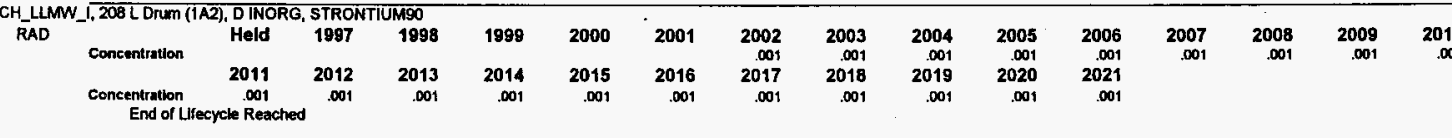

CH_LLMW_III End of Lifecycle Reached

\begin{tabular}{|c|c|c|c|c|c|c|c|c|c|c|}
\hline $\begin{array}{l}\text { Vol } \\
\text { Min\% } \\
\text { Max\% }\end{array}$ & Held & 1997 & 1998 & 1999 & 2000 & 2001 & $\begin{array}{r}2002 \\
40 \\
50 \\
200\end{array}$ & $\begin{array}{r}2003 \\
40 \\
50 \\
200\end{array}$ & $\begin{array}{r}2004 \\
40 \\
50 \\
200\end{array}$ & $\begin{array}{r}2005 \\
40 \\
50 \\
200\end{array}$ \\
\hline $\begin{array}{l}\text { Vol } \\
\text { Min\% } \\
\text { Max\% }\end{array}$ & $\begin{array}{r}2011 \\
200 \\
50 \\
200\end{array}$ & $\begin{array}{r}2012 \\
200 \\
50 \\
200\end{array}$ & $\begin{array}{r}2013 \\
200 \\
50 \\
200\end{array}$ & $\begin{array}{r}2014 \\
200 \\
50 \\
200\end{array}$ & $\begin{array}{r}2015 \\
200 \\
50 \\
200\end{array}$ & $\begin{array}{r}2016 \\
200 \\
50 \\
200\end{array}$ & $\begin{array}{r}2017 \\
200 \\
50 \\
200\end{array}$ & $\begin{array}{r}2018 \\
200 \\
50 \\
200\end{array}$ & $\begin{array}{r}2019 \\
200 \\
50 \\
200\end{array}$ & $\begin{array}{r}2020 \\
200 \\
50 \\
200\end{array}$ \\
\hline
\end{tabular}

$\begin{array}{rrrrr}2006 & 2007 & 2008 & 2009 & 2010 \\ 10 & 40 & 40 & 40 & 40 \\ 50 & 50 & 50 & 50 & 50 \\ 200 & 200 & 200 & 200 & 200\end{array}$

CH_LLMW_III, 208 L ORum (1A2) Container

Percentupe
Cont. Count

Percentuge
Cont. Count

)

\begin{tabular}{llll}
\hline 997 & $1998 \quad 1999 \quad 2000$
\end{tabular}

$2000 \quad 2001$

$2002 \quad 2003 \quad 2004$ Percent Full:

2021
200
50
200

\begin{tabular}{|c|c|c|c|c|c|c|c|c|c|c|c|c|c|c|c|c|}
\hline \multicolumn{17}{|c|}{ CH_LLMW_IIT, 208 L Drum (1A2), D INORG } \\
\hline PWF & Percentage & & & & & & & $\begin{array}{r}2002 \\
100\end{array}$ & $\begin{array}{r}2003 \\
100\end{array}$ & $\begin{array}{r}2004 \\
100\end{array}$ & $\begin{array}{r}2005 \\
100\end{array}$ & $\begin{array}{r}2006 \\
100\end{array}$ & $\begin{array}{r}2007 \\
100\end{array}$ & $\begin{array}{r}2008 \\
100\end{array}$ & $\begin{array}{r}2009 \\
900\end{array}$ & $\begin{array}{r}2010 \\
100\end{array}$ \\
\hline & & 2011 & 2012 & 2013 & 2014 & 2015 & 2016 & 2017 & 2018 & 2019 & 2020 & 2021 & & & & \\
\hline 7 & $\begin{array}{l}\text { Parcentage } \\
\text { End of }\end{array}$ & $\begin{array}{r}100 \\
\text { ycle Reac }\end{array}$ & 100 & 100 & 100 & 100 & 100 & 100 & 100 & 100 & 100 & 100 & & & & \\
\hline
\end{tabular}


Waste Generator

Waste Class, Container, Physical Waste Form, (Radionuclide or Hazardous Constituent)

Waste Clase, Container, Physical Waste Form, (Radionuclide or Hazardous Constituent)

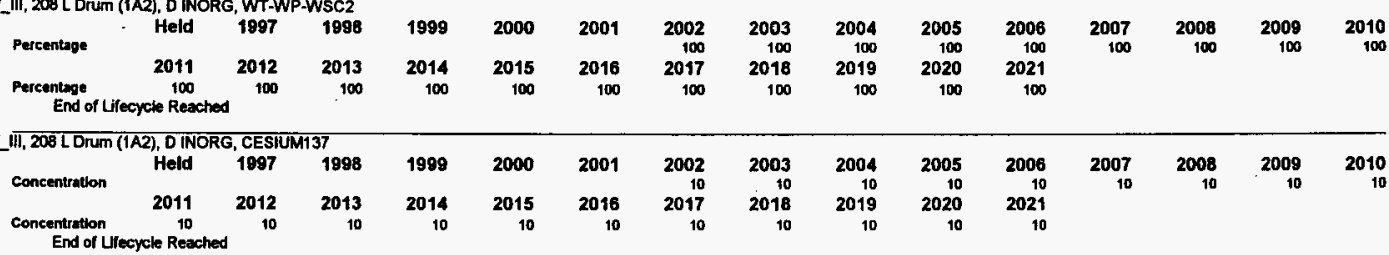

CH_LLMW_III, 208 L Drum (1A2), DINORG, CESIUM13) RAD End of Ufecycle Reached

\begin{tabular}{|c|c|c|c|c|c|c|c|c|c|c|c|c|c|c|c|c|}
\hline CH_LLM & III, $20 \mathrm{~B}$ L Drum & (2), DINC & G, STRO & UMSO & & & & & & & & & & & & \\
\hline RAD & Concentration & $\begin{array}{l}\text { Hold } \\
2011\end{array}$ & $\begin{array}{l}1897 \\
2012\end{array}$ & $\begin{array}{r}1998 \\
2013\end{array}$ & $\begin{array}{r}1998 \\
2014\end{array}$ & $\begin{array}{r}2000 \\
2015\end{array}$ & $\begin{array}{r}2001 \\
2016\end{array}$ & $\begin{array}{r}2002 \\
10 \\
2017\end{array}$ & $\begin{array}{r}2003 \\
10 \\
2018\end{array}$ & $\begin{array}{r}2004 \\
10 \\
2019\end{array}$ & $\begin{array}{r}2005 \\
10 \\
2020\end{array}$ & $\begin{array}{r}2006 \\
10 \\
2021\end{array}$ & $\begin{array}{r}2007 \\
10\end{array}$ & $\begin{array}{r}2008 \\
10\end{array}$ & $\begin{array}{r}2009 \\
10\end{array}$ & $\begin{array}{r}2010 \\
10\end{array}$ \\
\hline & $\begin{array}{l}\text { Concentration } \\
\text { End of Lif }\end{array}$ & 10 & 10 & 10 & 10 & 10 & 10 & 10 & 10 & 10 & 10 & 10 & & & & \\
\hline
\end{tabular}

HAZ End of Lifecycle Reached

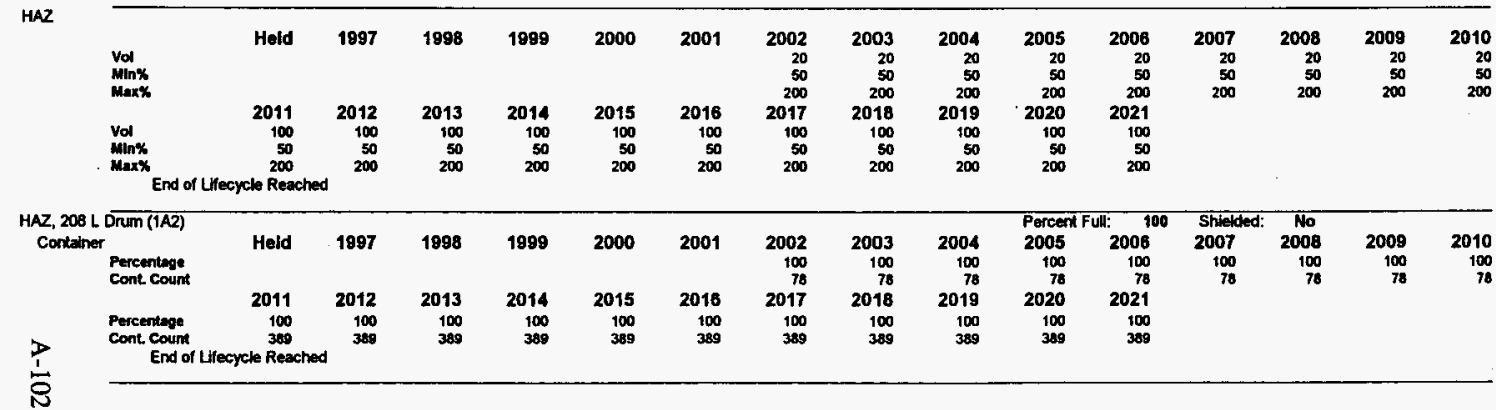


Waste Generator

. Waste Class, Container, Physical Waste Form, (Radionucllde or Hazardous Constituent)

PWF

$\begin{array}{lrr} & \text { Held } & 1997 \\ \text { Percentuge } & & \\ & 2011 & 20 \\ \text { Percentage } & 100 & \end{array}$

$\begin{array}{llllll}1998 & 1999 & 2000 & 2001 & 2002 & 2003\end{array}$

2003
100
2018

2004
100
2019

$\begin{array}{rrr}100 & 2005 & 2006 \\ & 100 & 500\end{array}$

$\begin{array}{rr}2005 & 2006 \\ 100 & 500 \\ 2020 & 2021\end{array}$

2007

2008

$2009 \quad 2010$

100

100

\begin{tabular}{|c|c|c|c|c|c|c|c|c|c|c|c|c|c|c|c|c|}
\hline \multirow{2}{*}{\multicolumn{17}{|c|}{$\begin{array}{l}\text { HAZ, } 208 \text { L D DUm (1A2), D INORG, WT-WP-WSC2 } \\
\text { HC }\end{array}$}} \\
\hline & & & & 1998 & 1999 & 2000 & 2001 & 2002 & 2003 & 2004 & 2005 & 2006 & 2007 & 2008 & 2009 & 2010 \\
\hline \multirow{2}{*}{ HC } & Percentuge & 2011 & 2012 & 2013 & 2014 & 2015 & 2016 & $\begin{array}{r}100 \\
2017\end{array}$ & $\begin{array}{r}100 \\
2018\end{array}$ & $\begin{array}{r}100 \\
2019\end{array}$ & $\begin{array}{r}100 \\
2020\end{array}$ & $\begin{array}{r}100 \\
2021\end{array}$ & 100 & 100 & 100 & 100 \\
\hline & $\begin{array}{l}\text { Parcentage } \\
\text { End of }\end{array}$ & $\begin{array}{l}100 \\
\text { cle Reac }\end{array}$ & 100 & 100 & 100 & 100 & 100 & 100 & 100 & 100 & 100 & 100 & & & & \\
\hline
\end{tabular}

息 
Waste Generator

Waste Clase, Container, Physical Waste Form, (Radionuclide or Hazardous Constituent)

WHC SST LLE End of Llecycle: 202

\begin{tabular}{|c|c|c|c|c|c|c|c|c|c|c|c|c|c|c|c|}
\hline $\begin{array}{l}\text { Vol } \\
\text { Min\% }\end{array}$ & Held & 1997 & 1998 & 1999 & 2000 & $\begin{array}{r}2001 \\
590 \\
50 \\
133\end{array}$ & $\begin{array}{r}2002 \\
657 \\
50 \\
133\end{array}$ & $\begin{array}{r}2003 \\
68 \\
50 \\
133\end{array}$ & $\begin{array}{r}2004 \\
571 \\
50 \\
133\end{array}$ & $\begin{array}{r}2005 \\
1.127 \\
50 \\
133\end{array}$ & $\begin{array}{r}2006 \\
763 \\
50 \\
133\end{array}$ & $\begin{array}{r}2007 \\
1,003 \\
50 \\
133\end{array}$ & $\begin{array}{r}2008 \\
1,584 \\
50 \\
133\end{array}$ & $\begin{array}{r}2009 \\
2.109 \\
50 \\
133\end{array}$ & $\begin{array}{r}2010 \\
2,062 \\
50 \\
133\end{array}$ \\
\hline & 2011 & 2012 & $\begin{array}{r}2013 \\
2524\end{array}$ & $\begin{array}{r}2014 \\
2750\end{array}$ & 2015 & $\begin{array}{r}2016 \\
1077\end{array}$ & 2017 & 2018 & 2019 & 2020 & 2021 & 2022 & 2023 & 2024 & \\
\hline
\end{tabular}

$\begin{array}{lrrrrrrr} & 2011 & 2012 & 2013 & 2014 & 2015 & 2016 & 2017 \\ \text { Vot } & 2,451 & 1,857 & 2,524 & 2,750 & 538 & 1,077 & 546 \\ \text { Min\% } & 50 & 50 & 50 & -50 & 50 & 50 & 50 \\ \text { Max* } & 133 & 139 & 133 & 133 & 133 & 133 & 133 \\ \text { End of Lfecycle Reached } & & & & & & \end{array}$

End of Lifecycla Reached

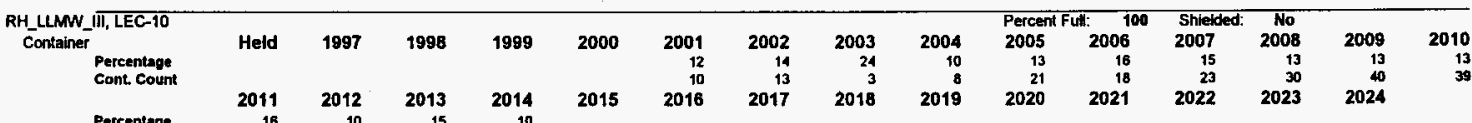

Pereentoge

End of Lffecycle Reached

\begin{tabular}{|c|c|c|c|c|c|c|c|c|c|c|c|c|c|c|c|c|}
\hline PWF & $\begin{array}{l}\text { Percentage } \\
\text { Percentage } \\
\text { End of }\end{array}$ & $\begin{array}{r}\text { Held } \\
2011 \\
100 \\
\text { cle Rea }\end{array}$ & $\begin{array}{r}1997 \\
2012 \\
100\end{array}$ & $\begin{array}{r}1998 \\
2013 \\
100\end{array}$ & $\begin{array}{r}1999 \\
2014 \\
100\end{array}$ & $\begin{array}{l}2000 \\
2015\end{array}$ & $\begin{array}{r}2001 \\
100 \\
2016\end{array}$ & $\begin{array}{r}2002 \\
100 \\
2017\end{array}$ & $\begin{array}{r}2003 \\
100 \\
2018\end{array}$ & $\begin{array}{r}2004 \\
100 \\
2019\end{array}$ & $\begin{array}{r}2005 \\
100 \\
2020\end{array}$ & $\begin{array}{r}2006 \\
100 \\
2021\end{array}$ & $\begin{array}{r}2007 \\
100 \\
2022\end{array}$ & $\begin{array}{r}2008 \\
100 \\
2023\end{array}$ & $\begin{array}{r}2009 \\
100 \\
2024\end{array}$ & $\begin{array}{r}2010 \\
\quad 100\end{array}$ \\
\hline${ }_{\text {HC }}^{R H}$ & $\begin{array}{l}\text { III, LEC-10, } \\
\text { Percentage } \\
\text { Percentage } \\
\text { End of }\end{array}$ & $\begin{array}{r}\text { AL-COI } \\
\text { Held } \\
2011 \\
100 \\
\text { cle Rea }\end{array}$ & $\begin{array}{r}\text { NT-WP } \\
1997 \\
2012 \\
100\end{array}$ & $\begin{array}{r}1898 \\
2013 \\
100\end{array}$ & $\begin{array}{r}1999 \\
2014 \\
100\end{array}$ & $\begin{array}{l}2000 \\
2015\end{array}$ & $\begin{array}{r}2001 \\
100 \\
2016\end{array}$ & $\begin{array}{r}2002 \\
100 \\
2017\end{array}$ & $\begin{array}{r}2003 \\
100 \\
2018\end{array}$ & $\begin{array}{r}2004 \\
100 \\
2019\end{array}$ & $\begin{array}{r}2005 \\
100 \\
2020\end{array}$ & $\begin{array}{r}2006 \\
100 \\
2021\end{array}$ & $\begin{array}{r}2007 \\
100 \\
2022\end{array}$ & $\begin{array}{r}2008 \\
100 \\
2023\end{array}$ & $\begin{array}{r}2009 \\
100 \\
2024\end{array}$ & $\begin{array}{r}2010 \\
100\end{array}$ \\
\hline
\end{tabular}

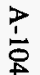


Waste Generator

Waste Class, Container, Physical Waste Form, (Radionuclide or Hazardous Constifuent)

WH_LLIWW_III, LEC-10, D METAL-CONT, CESIUMI37

\begin{tabular}{|c|c|c|c|c|c|c|c|c|c|c|c|c|c|c|c|c|}
\hline RAD & $\begin{array}{l}\text { Concentriation } \\
\text { Concenturation } \\
\text { End of Ufe }\end{array}$ & $\begin{array}{l}\text { Held } \\
2011 \\
100 \\
\text { vele Reached }\end{array}$ & $\begin{array}{r}1997 \\
2012 \\
100\end{array}$ & $\begin{array}{r}1998 \\
2013 \\
100\end{array}$ & $\begin{array}{r}1890 \\
2014 \\
100\end{array}$ & $\begin{array}{l}2000 \\
2015\end{array}$ & $\begin{array}{r}2001 \\
100 \\
2016\end{array}$ & $\begin{array}{r}2002 \\
100 \\
2017\end{array}$ & $\begin{array}{r}2003 \\
100 \\
2018\end{array}$ & $\begin{array}{r}2004 \\
100 \\
2019\end{array}$ & $\begin{array}{r}2005 \\
100 \\
2020\end{array}$ & $\begin{array}{r}2006 \\
100 \\
2021\end{array}$ & $\begin{array}{r}2007 \\
100 \\
2022\end{array}$ & $\begin{array}{r}2008 \\
100 \\
2023\end{array}$ & $\begin{array}{r}2009 \\
100 \\
2024\end{array}$ & $\begin{array}{r}2010 \\
100\end{array}$ \\
\hline$\underset{\text { RAD }}{R H L L M W}$ & $\begin{array}{l}\text { III, LEC-10, DME } \\
\text { Concentration } \\
\text { Concentration } \\
\text { End of Life }\end{array}$ & $\begin{array}{l}\text { AL-CONT, } \\
\text { Held } \\
2011 \\
100 \\
\text { pele Reached }\end{array}$ & $\begin{array}{r}1997 \\
2012 \\
100\end{array}$ & $\begin{array}{r}190 \\
1998 \\
2013 \\
100 \\
\end{array}$ & $\begin{array}{r}1989 \\
2014 \\
100\end{array}$ & $\begin{array}{l}2000 \\
2015\end{array}$ & $\begin{array}{r}2001 \\
100 \\
2016\end{array}$ & $\begin{array}{r}2002 \\
100 \\
2017\end{array}$ & $\begin{array}{r}2003 \\
100 \\
2018\end{array}$ & $\begin{array}{r}2004 \\
100 \\
2019\end{array}$ & $\begin{array}{r}2005 \\
100 \\
2020\end{array}$ & $\begin{array}{r}2006 \\
100 \\
2021\end{array}$ & $\begin{array}{r}2007 \\
100 \\
2022\end{array}$ & $\begin{array}{r}2008 \\
100 \\
2023\end{array}$ & $\begin{array}{r}2009 \\
100 \\
2024\end{array}$ & $\begin{array}{r}2010 \\
100\end{array}$ \\
\hline $\begin{array}{c}\text { RH_LLMW } \\
\text { Container }\end{array}$ & $\begin{array}{l}\text { 11i, LEC-11 } \\
\text { Percentage } \\
\text { Cont. Count } \\
\text { Percentage } \\
\text { Cont. Count } \\
\text { End of Life }\end{array}$ & $\begin{array}{l}\text { Held } \\
2011 \\
20 \\
20 \\
\text { clo Reached }\end{array}$ & $\begin{array}{r}1997 \\
2012 \\
5 \\
9\end{array}$ & $\begin{array}{r}1988 \\
2013 \\
7 \\
19\end{array}$ & $\begin{array}{r}1999 \\
\\
2014 \\
5 \\
15\end{array}$ & $\begin{array}{l}2000 \\
2015\end{array}$ & $\begin{array}{r}2001 \\
21 \\
13 \\
2016\end{array}$ & $\begin{array}{r}2002 \\
7 \\
5 \\
2017\end{array}$ & $\begin{array}{r}2003 \\
11 \\
1 \\
2018\end{array}$ & $\begin{array}{r}2004 \\
5 \\
3 \\
2019\end{array}$ & $\begin{array}{c}\text { Percent } \\
2005 \\
6 \\
7 \\
2020\end{array}$ & $\begin{array}{c}\text { U: } 100 \\
2006 \\
7 \\
6 \\
2021\end{array}$ & $\begin{array}{c}\text { Shilided: } \\
2007 \\
7 \\
8 \\
2022\end{array}$ & $\begin{array}{r}\text { No } \\
2008 \\
6 \\
10 \\
2023 \\
\end{array}$ & $\begin{array}{r}2009 \\
7 \\
15 \\
2024\end{array}$ & $\begin{array}{r}2010 \\
8 \\
14\end{array}$ \\
\hline $\begin{array}{l}\text { RH_LLMW } \\
\text { PWF }\end{array}$ & $\begin{array}{l}\text { III, LEC-11, D ME } \\
\text { Percentuge } \\
\text { Percentege } \\
\text { End of Life }\end{array}$ & $\begin{array}{l}\text { AL-CONT } \\
\text { Held } \\
2011 \\
100 \\
\text { cle Reached }\end{array}$ & $\begin{array}{r}1997 \\
2012 \\
100\end{array}$ & $\begin{array}{r}1998 \\
2013 \\
100\end{array}$ & $\begin{array}{r}1999 \\
2014 \\
100\end{array}$ & $\begin{array}{l}2000 \\
2015\end{array}$ & $\begin{array}{r}2001 \\
100 \\
2016\end{array}$ & $\begin{array}{r}2002 \\
100 \\
2017\end{array}$ & $\begin{array}{r}2003 \\
100 \\
2018\end{array}$ & $\begin{array}{r}2004 \\
100 \\
2019\end{array}$ & $\begin{array}{r}2005 \\
100 \\
2020\end{array}$ & $\begin{array}{r}2006 \\
100 \\
2021\end{array}$ & $\begin{array}{r}2007 \\
100 \\
2022\end{array}$ & $\begin{array}{r}2008 \\
100 \\
2023\end{array}$ & $\begin{array}{r}2009 \\
100 \\
2024\end{array}$ & $\begin{array}{r}2010 \\
100\end{array}$ \\
\hline $\mathrm{RH}_{\mathrm{HC}}$ & $\begin{array}{l}\text { (II, LEC-11, D ME } \\
\text { Percentege } \\
\text { Percentege } \\
\text { End of Life }\end{array}$ & $\begin{array}{l}\text { AL-CONT, V } \\
\text { Held } \\
2011 \\
100 \\
\text { cheached }\end{array}$ & $\begin{array}{r}\text { NT-WP- } \\
1997 \\
2012 \\
100\end{array}$ & $\begin{array}{r}1998 \\
2013 \\
\text { t00 }\end{array}$ & $\begin{array}{r}1999 \\
2014 \\
100\end{array}$ & $\begin{array}{l}2000 \\
2015\end{array}$ & $\begin{array}{r}2001 \\
100 \\
2016\end{array}$ & $\begin{array}{r}2002 \\
100 \\
2017\end{array}$ & $\begin{array}{r}2003 \\
100 \\
2018\end{array}$ & $\begin{array}{r}2004 \\
100 \\
2019\end{array}$ & $\begin{array}{r}2005 \\
100 \\
2020\end{array}$ & $\begin{array}{r}2006 \\
100 \\
2021\end{array}$ & $\begin{array}{r}2007 \\
100 \\
2022\end{array}$ & $\begin{array}{r}2008 \\
100 \\
2023\end{array}$ & $\begin{array}{r}2000 \\
100 \\
2024\end{array}$ & $\begin{array}{r}2010 \\
100\end{array}$ \\
\hline
\end{tabular}

$\frac{7}{8}$ 


\section{Waste Generator}

Waste Class, Container, Phyclical Waste Form, (Radionuclide or Hazardous Constituent)

AH_LIMW_II, LEC-11, DMETAL-CONT, CESIUMIJ7

RAD

\begin{tabular}{ccc|} 
& Held & 1997 \\
Concentration & & \\
& 2011 & 2012 \\
Concentration & 100 & 100 \\
End of Lifecycle Reached & & 201 \\
\hline
\end{tabular}

1998
2013
100

1999
2014
100

2000

2001
100
2016

$\begin{array}{rr}2002 & 2003 \\ 100 & 100 \\ 2017 & 2018\end{array}$

2004
100
2019

RH_LLMW_III, LEC-11, D METAL-CONT, STTRONTIUMMSO

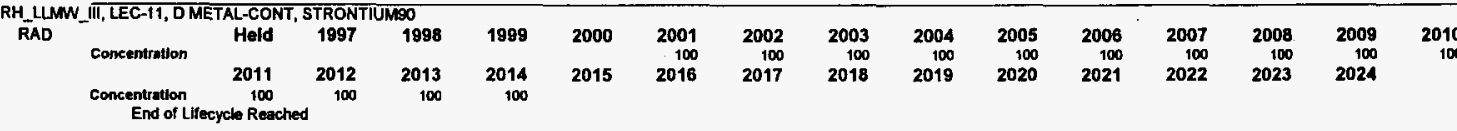

\begin{tabular}{|c|c|c|c|c|c|c|c|c|c|c|c|c|c|c|c|}
\hline \multirow{4}{*}{$\begin{array}{r}\text { RH_LLMW_III, LEC-12 } \\
\text { Container } \\
\text { Percentage } \\
\text { Cont. Count }\end{array}$} & & & & & & & & & & Percen & 10 & Shielded: & No & & \\
\hline & Held & 1897 & 1998 & 1999 & 2000 & 2001 & 2002 & 2003 & 2004 & 2005 & 2006 & 2007 & 2008 & 2009 & 2010 \\
\hline & & & & & & & & & 12 & & & 5 & & & 7 \\
\hline & 2011 & 2012 & 2013 & 2014 & 2015 & 2016 & $2017^{1}$ & 2018 & $2019^{5}$ & $2020^{6}$ & $2021^{2}$ & 2022 & 2023 & 2024 & \\
\hline $\begin{array}{l}\text { Percentage } \\
\text { Cont. Count }\end{array}$ & 7 & $\begin{array}{l}11 \\
16\end{array}$ & 9 & $\begin{array}{l}11 \\
23\end{array}$ & 25 & $\begin{array}{l}25 \\
20\end{array}$ & 25 & & & & & & & & \\
\hline
\end{tabular}
End of Lifecycte Reached

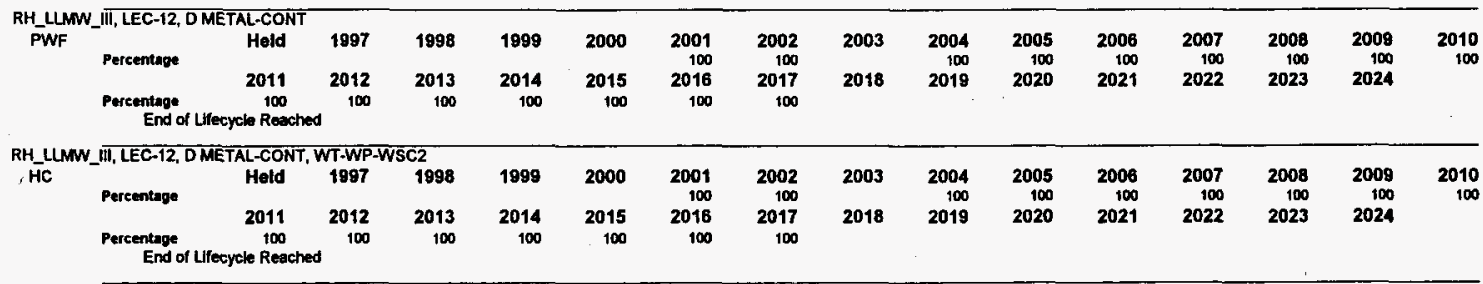

$\frac{2}{8}$ 
Waste Generator

Waste Class, Container, Physical Waste Form, (Radionuclide or Hazardous Constituent)

RH_LLLWW_III, LEC-12, D METAL-CONT, CESIUMI37

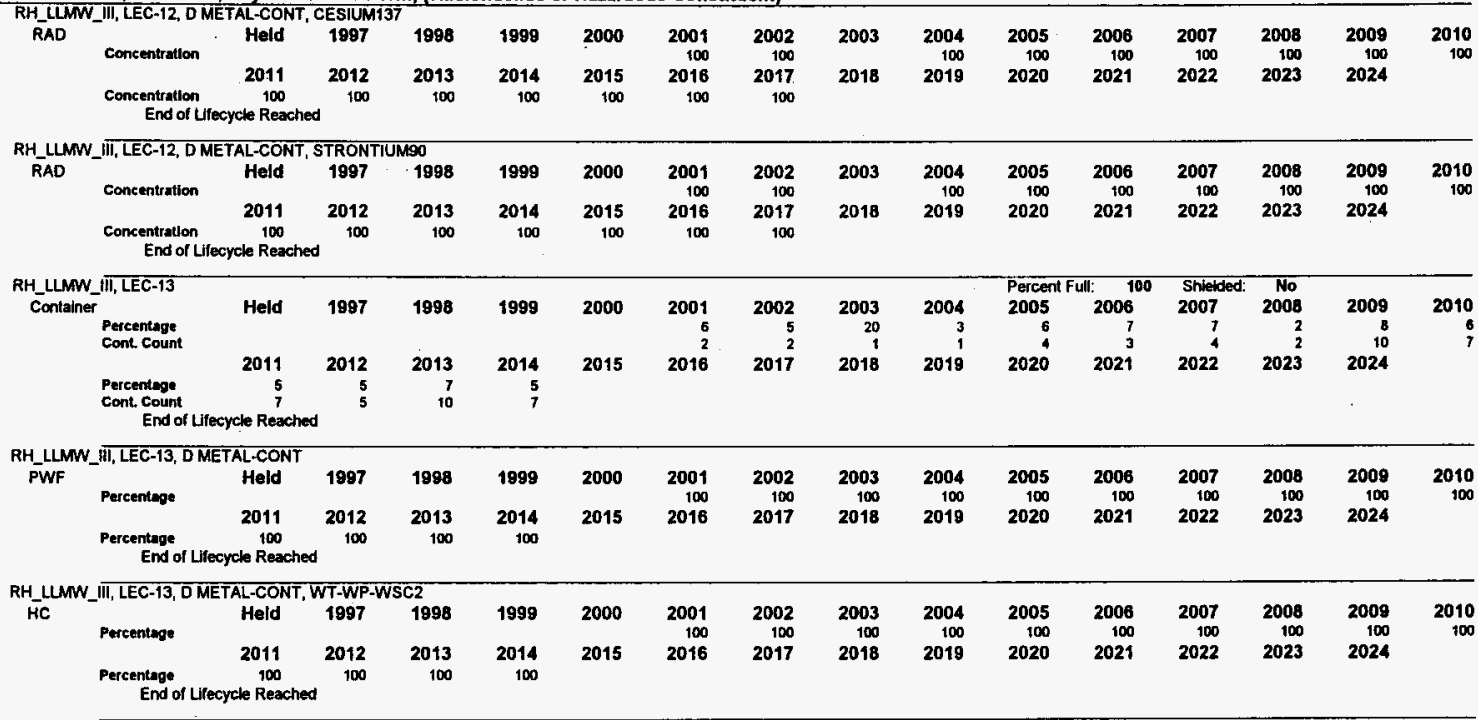


Waste Generator

Waste Class, Container, Physical Waste Form, (Radionuclide or Hazardous Constituent)

\begin{tabular}{|c|c|c|c|c|c|c|c|c|c|c|c|c|c|c|c|c|}
\hline $\begin{array}{c}\text { RH_LLMW } \\
\text { RAD }\end{array}$ & $\begin{array}{l}\text { III, LEC-13, D M } \\
\text { Concentration } \\
\text { Concentration } \\
\text { End of Life }\end{array}$ & $\begin{array}{l}\text { TAL-CONT, } \\
\text { Held } \\
2011 \\
100 \\
\text { ycle Reache }\end{array}$ & $\begin{array}{r}1997 \\
2012 \\
100\end{array}$ & $\begin{array}{r}1998 \\
2013 \\
100\end{array}$ & $\begin{array}{r}1999 \\
2014 \\
100\end{array}$ & $\begin{array}{l}2000 \\
2015\end{array}$ & $\begin{array}{r}2001 \\
100 \\
2016\end{array}$ & $\begin{array}{r}2002 \\
100 \\
2017\end{array}$ & $\begin{array}{r}2003 \\
100 \\
2018\end{array}$ & $\begin{array}{r}2004 \\
100 \\
2019\end{array}$ & $\begin{array}{r}2005 \\
100 \\
2020\end{array}$ & $\begin{array}{r}2006 \\
100 \\
2021\end{array}$ & $\begin{array}{r}2007 \\
100 \\
2022\end{array}$ & $\begin{array}{r}2008 \\
100 \\
2023\end{array}$ & $\begin{array}{r}2009 \\
100 \\
2024\end{array}$ & $\begin{array}{r}2010 \\
100\end{array}$ \\
\hline$\underset{\text { RAD }}{\text { RH_LLMW }}$ & $\begin{array}{l}\text { III, LEC-13, DME } \\
\text { Concentration } \\
\text { Concentration } \\
\text { End of Life }\end{array}$ & $\begin{array}{l}\text { ALL-CONT, } \\
\text { Held } \\
2011 \\
100 \\
\text { Ycle Reached }\end{array}$ & $\begin{array}{r}\text { STRONT } \\
1997 \\
2012 \\
100\end{array}$ & $\begin{array}{r}1998 \\
2013 \\
100\end{array}$ & $\begin{array}{r}1999 \\
2014 \\
100\end{array}$ & $\begin{array}{l}2000 \\
2015\end{array}$ & $\begin{array}{r}2001 \\
100 \\
2016\end{array}$ & $\begin{array}{r}2002 \\
100 \\
2017\end{array}$ & $\begin{array}{r}2003 \\
100 \\
2018\end{array}$ & $\begin{array}{r}2004 \\
100 \\
2019\end{array}$ & $\begin{array}{r}2005 \\
100 \\
2020\end{array}$ & $\begin{array}{r}2006 \\
100 \\
2021\end{array}$ & $\begin{array}{r}2007 \\
100 \\
2022\end{array}$ & $\begin{array}{r}2008 \\
100 \\
2023\end{array}$ & $\begin{array}{r}2009 \\
100 \\
2024\end{array}$ & $\begin{array}{r}2010 \\
100\end{array}$ \\
\hline RH_LLMW, & $\begin{array}{l}\sqrt{111, \text { LEC-14 }} \\
\text { Percentage } \\
\text { Cont. Count } \\
\text { Percentuge } \\
\text { Conk. Count } \\
\text { End of Life }\end{array}$ & $\begin{array}{l}\text { Held } \\
2011 \\
34 \\
21 \\
\text { cle Reached }\end{array}$ & $\begin{array}{r}1997 \\
2012 \\
30 \\
14\end{array}$ & $\begin{array}{r}1998 \\
2013 \\
45 \\
29\end{array}$ & $\begin{array}{r}1999 \\
2014 \\
32 \\
22\end{array}$ & $\begin{array}{l}2000 \\
2015\end{array}$ & $\begin{array}{r}2001 \\
34 \\
5 \\
2016\end{array}$ & $\begin{array}{r}2002 \\
43 \\
7 \\
2017\end{array}$ & $\begin{array}{r}2003 \\
46 \\
1 \\
2018\end{array}$ & $\begin{array}{r}2004 \\
28 \\
4 \\
2019\end{array}$ & $\begin{array}{c}\text { Percent } \\
2005 \\
39 \\
11 \\
2020\end{array}$ & $\begin{array}{c}100 \\
2006 \\
51 \\
10 \\
2021\end{array}$ & $\begin{array}{c}\text { Shielded: } \\
2007 \\
44 \\
12 \\
2022\end{array}$ & $\begin{array}{r}\text { No } \\
2008 \\
43 \\
17 \\
2023\end{array}$ & $\begin{array}{r}2009 \\
38 \\
20 \\
2024\end{array}$ & $\begin{array}{r}2010 \\
40 \\
21\end{array}$ \\
\hline$\underset{\text { PWF }}{R H_{\text {_L }} L M W}$ & $\begin{array}{l}\text { III, LEC-14, D ME } \\
\text { Percentage } \\
\text { Percentage } \\
\text { End of Life }\end{array}$ & $\begin{array}{l}\text { AL-CONT } \\
\text { Held } \\
2011 \\
100 \\
\text { pcle Reacher }\end{array}$ & $\begin{array}{r}1997 \\
2012 \\
100\end{array}$ & $\begin{array}{r}1998 \\
2013 \\
100\end{array}$ & $\begin{array}{r}1999 \\
2014 \\
100\end{array}$ & $\begin{array}{l}2000 \\
2015\end{array}$ & $\begin{array}{r}2001 \\
100 \\
2016\end{array}$ & $\begin{array}{r}2002 \\
100 \\
2017\end{array}$ & $\begin{array}{r}2003 \\
100 \\
2018\end{array}$ & $\begin{array}{r}2004 \\
100 \\
2019\end{array}$ & $\begin{array}{r}2005 \\
100 \\
2020\end{array}$ & $\begin{array}{r}2006 \\
100 \\
2021\end{array}$ & $\begin{array}{r}2007 \\
100 \\
2022\end{array}$ & $\begin{array}{r}2008 \\
100 \\
2023\end{array}$ & $\begin{array}{r}2009 \\
100 \\
2024\end{array}$ & $\begin{array}{r}2010 \\
100\end{array}$ \\
\hline $\mathrm{RH}_{\mathrm{HC}}^{\mathrm{RH} L \mathrm{~L} M \mathrm{w}_{-}}$ & $\begin{array}{l}\text { III, LEC-14, DME } \\
\text { Percentage } \\
\text { Percentage } \\
\text { End of Life }\end{array}$ & $\begin{array}{l}\text { AL-CONT, } \\
\text { Held } \\
2011 \\
100 \\
\text { rcle Reached }\end{array}$ & $\begin{array}{r}\text { NT-WP-Y } \\
1997 \\
2012 \\
100\end{array}$ & $\begin{array}{r}\bar{C} 2 \\
1998 \\
2013 \\
100\end{array}$ & $\begin{array}{r}1989 \\
2014 \\
100\end{array}$ & $\begin{array}{l}2000 \\
2015\end{array}$ & $\begin{array}{r}2001 \\
100 \\
2016\end{array}$ & $\begin{array}{r}2002 \\
100 \\
2017\end{array}$ & $\begin{array}{r}2003 \\
100 \\
2018\end{array}$ & $\begin{array}{r}2004 \\
100 \\
2019\end{array}$ & $\begin{array}{r}2005 \\
100 \\
2020\end{array}$ & $\begin{array}{r}2006 \\
100 \\
2021\end{array}$ & $\begin{array}{r}2007 \\
100 \\
2022\end{array}$ & $\begin{array}{r}2008 \\
100 \\
2023\end{array}$ & $\begin{array}{r}2009 \\
100 \\
2024\end{array}$ & $\begin{array}{r}2010 \\
100\end{array}$ \\
\hline
\end{tabular}

$\frac{p}{8}$ 
Waste Generator

Waste Class, Container, Physical Waste Form, (Radionuclide or Hazardous Constltuent)

RH_LLMW_ _ii, LEC-14, D METAL-CONT, CESIUM137

RAD

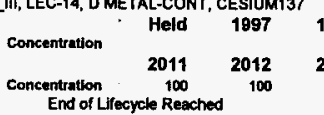

$1998 \quad 1989$

$2000 \quad 2001 \quad 2002$

$2002 \quad 2003$

2003
100

$2004 \quad 2005$

$\begin{array}{rrr}2008 & 2009 & 2010 \\ 100 & 100 & 100\end{array}$

$2014 \quad 2015 \quad 2018$

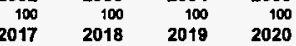

$\begin{array}{rrrrr}2000 & 2007 & 2008 & 2009 & 2010 \\ 100 & 100 & 100 & 100\end{array}$

End of Lifecycle Reached

RH_LLMW_III, LEC-14, D METAL-CONT, STRONTIUMSO

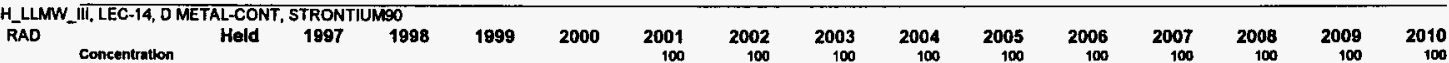

$2011 \quad 2012 \quad 2013 \quad 2014$

End of Lifecycle Reached

RH_LLMW
Container

Percentage

\begin{tabular}{rrrrrrr} 
& 2011 & 2012 & 2013 & 2014 & 2015 & 2016 \\
Percentage & 30 & 39 & 16 & 37 & 75 & \\
\hline
\end{tabular}

Cont checycle Reached

RH_LLMW_III, LEC-15, D METAL-CONT

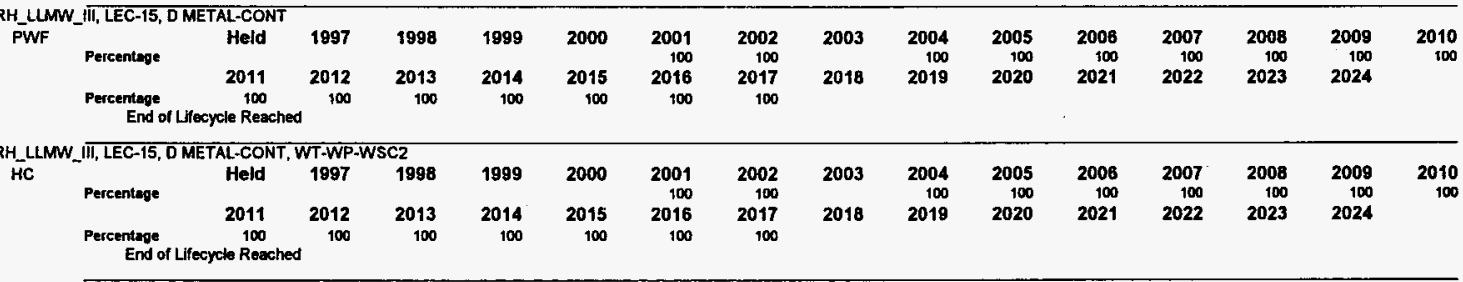

$\frac{7}{1}$ 
Waste Generator

Waste Class, Contalner, Physical Waste Form, (Radionuclide or Hazardous Constituent)

RH_LLMW_III, LEC-15, D METAL-CONT, CESIUM137

RAD

\begin{tabular}{ccc|} 
Concentration & Held & 1997 \\
& 2011 & 2012 \\
Concentration & 100 & 100 \\
End of Lifecycle Reached
\end{tabular}

$199819992000 \quad 2001$

End of Lifecycle Reached

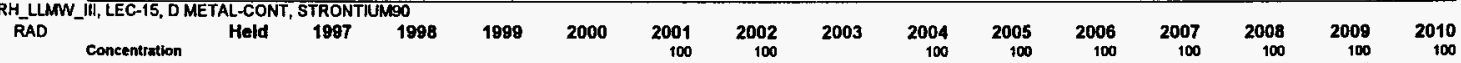

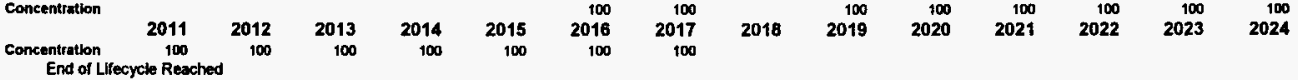
End of Lifecycle Reached

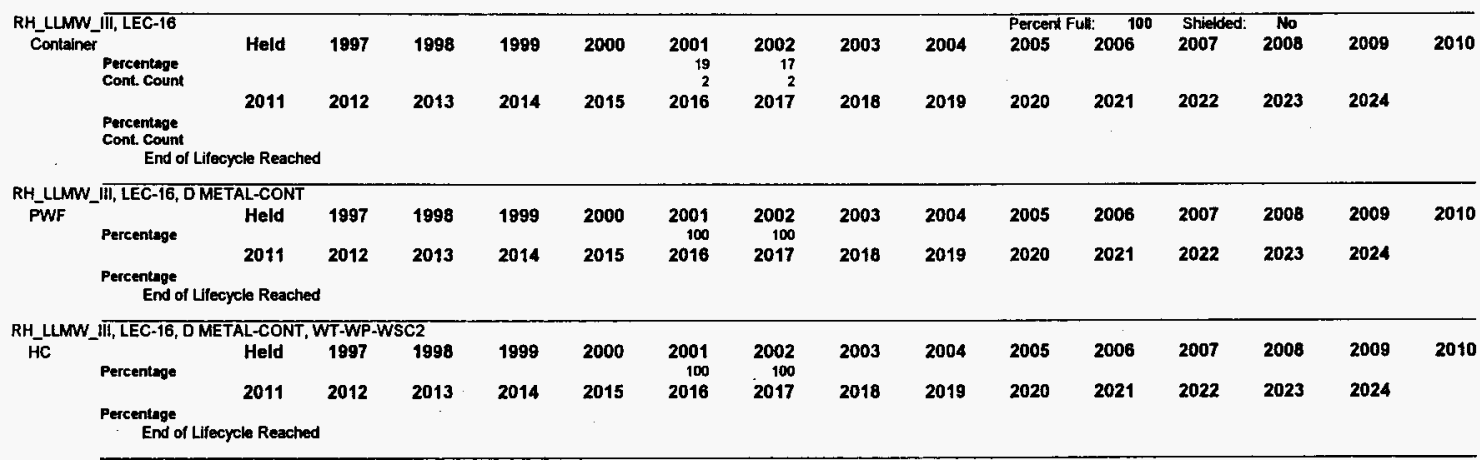

$\stackrel{\stackrel{1}{\rightleftarrows}}{\stackrel{D}{0}}$ 
Waste Generator

Waste Class, Container, Physical Waste Form, (Radionuclide or Hazardous Constituent)

\begin{tabular}{|c|c|c|c|c|c|c|c|c|c|c|c|c|c|c|c|c|}
\hline RAD & $\begin{array}{l}\text { Concentration } \\
\text { Concentration } \\
\text { End of Lif }\end{array}$ & $\begin{array}{l}\text { Held } \\
2011\end{array}$ & 2012 & $\begin{array}{l}1998 \\
2013\end{array}$ & $\begin{array}{l}1999 \\
2014\end{array}$ & $\begin{array}{l}2000 \\
2015\end{array}$ & $\begin{array}{r}2001 \\
100 \\
2016\end{array}$ & $\begin{array}{r}2002 \\
100 \\
2017\end{array}$ & $\begin{array}{l}2003 \\
2018\end{array}$ & $\begin{array}{l}2004 \\
2019\end{array}$ & $\begin{array}{l}2005 \\
2020\end{array}$ & $\begin{array}{l}2006 \\
2021\end{array}$ & $\begin{array}{l}2007 \\
2022\end{array}$ & $\begin{array}{l}2008 \\
2023\end{array}$ & $\begin{array}{l}2009 \\
2024\end{array}$ & 2010 \\
\hline$\underset{\text { RAD }}{\text { RH_LLMW }}$ & $\begin{array}{l}\text { III, LEC-16, DM } \\
\text { Concentration } \\
\text { concentration } \\
\text { End of Lif }\end{array}$ & $\begin{array}{l}\text { AL-CONT } \\
\text { Held } \\
2011 \\
\text { cle Reach }\end{array}$ & $\begin{array}{l}\text { STRONTI } \\
1997 \\
2012\end{array}$ & $\begin{array}{l}190 \\
1998 \\
2013\end{array}$ & $\begin{array}{l}1999 \\
2014\end{array}$ & $\begin{array}{l}2000 \\
2015\end{array}$ & $\begin{array}{r}2001 \\
100 \\
2016\end{array}$ & $\begin{array}{r}2002 \\
100 \\
2017\end{array}$ & $\begin{array}{l}2003 \\
2018\end{array}$ & $\begin{array}{l}2004 \\
2019\end{array}$ & $\begin{array}{l}2005 \\
2020\end{array}$ & $\begin{array}{l}2006 \\
2021\end{array}$ & $\begin{array}{l}2007 \\
2022\end{array}$ & $\begin{array}{l}2008 \\
2023\end{array}$ & $\begin{array}{l}2009 \\
2024\end{array}$ & 2010 \\
\hline $\begin{array}{l}\text { RH_LLMW } \\
\text { Container }\end{array}$ & $\begin{array}{l}\text { Iit, LEC-18 } \\
\text { Percentage } \\
\text { Cont. Count } \\
\text { Percentage } \\
\text { Cont. Count } \\
\text { End of Lite }\end{array}$ & $\begin{array}{l}\text { Held } \\
2011\end{array}$ & $\begin{array}{l}1997 \\
2012\end{array}$ & 2013 & $\begin{array}{l}1999 \\
2014\end{array}$ & $\begin{array}{l}2000 \\
2015\end{array}$ & $\begin{array}{l}2001 \\
2016\end{array}$ & $\begin{array}{l}2002 \\
2017\end{array}$ & $\begin{array}{l}2003 \\
2018\end{array}$ & $\begin{array}{l}2004 \\
2019\end{array}$ & $\begin{array}{l}\text { Percent } \\
2005 \\
2020\end{array}$ & $\begin{array}{l}2006 \\
2021\end{array}$ & $\begin{array}{l}\text { Shieided: } \\
2007 \\
2022\end{array}$ & $\begin{array}{c}\text { No } \\
2008 \\
2023\end{array}$ & $\begin{array}{r}2009 \\
3 \\
1 \\
2024\end{array}$ & 2010 \\
\hline$\underset{\text { PWF }}{R \text { RHLIMW }}$ & $\begin{array}{l}\text { III, LEC-18, DM } \\
\text { Pereentage } \\
\text { Percentage } \\
\text { End of Life }\end{array}$ & $\begin{array}{l}\text { AL-CONT } \\
\text { Held } \\
2011 \\
\text { cle Reach }\end{array}$ & $\begin{array}{l}1997 \\
2012\end{array}$ & $\begin{array}{l}1998 \\
2013\end{array}$ & $\begin{array}{l}1999 \\
2014\end{array}$ & $\begin{array}{l}2000 \\
2015\end{array}$ & $\begin{array}{l}2001 \\
2016\end{array}$ & $\begin{array}{l}2002 \\
2017\end{array}$ & $\begin{array}{l}2003 \\
2018\end{array}$ & $\begin{array}{l}2004 \\
2019\end{array}$ & $\begin{array}{l}2005 \\
2020\end{array}$ & $\begin{array}{l}2006 \\
2021\end{array}$ & $\begin{array}{l}2007 \\
2022\end{array}$ & $\begin{array}{l}2008 \\
2023\end{array}$ & $\begin{array}{r}2009 \\
100 \\
2024\end{array}$ & 2010 \\
\hline$\underset{\text { HC }}{R H \text { ILMW }}$ & $\begin{array}{l}\text { III, LEC-18, O M } \\
\text { Percentage } \\
\text { Percentage } \\
\text { End of Life }\end{array}$ & $\begin{array}{l}\text { Al-CONT } \\
\text { Held } \\
2011 \\
\text { clo Reach }\end{array}$ & $\begin{array}{l}\text { NT-WP-V } \\
1997 \\
2012\end{array}$ & $\begin{array}{l}C 2 \\
1998 \\
2013\end{array}$ & $\begin{array}{l}1999 \\
2014\end{array}$ & $\begin{array}{l}2000 \\
2015\end{array}$ & $\begin{array}{l}2001 \\
2016\end{array}$ & $\begin{array}{l}2002 \\
2017\end{array}$ & $\begin{array}{l}2003 \\
2018\end{array}$ & $\begin{array}{l}2004 \\
2019\end{array}$ & $\begin{array}{l}2005 \\
2020\end{array}$ & $\begin{array}{l}2006 \\
2021\end{array}$ & $\begin{array}{l}2007 \\
2022\end{array}$ & $\begin{array}{l}2008 \\
2023\end{array}$ & $\begin{array}{r}2009 \\
100 \\
2024\end{array}$ & 2010 \\
\hline
\end{tabular}

5
$\square$
$\square$ 
Waste Generator

Waste Class, Container, Phyaical Waste Form, (Radionuclide or Hazardous Constituent)

RH_LLMW_III, LEC-18, D METAL-CONT, CESIUMI RAD

concentration.

Held 1997

Concentratton

20112012

End of Ltecycle Reached

RH_LLMW_III, LEC-18, D METAL-CONT, STRONTIUMEO

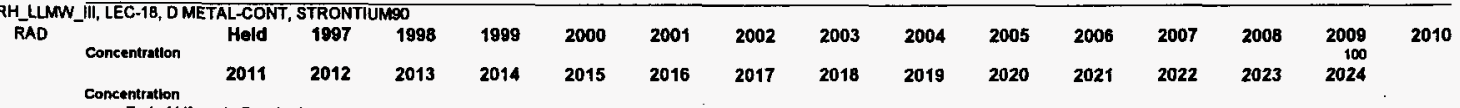
End of Lifecycle Reached

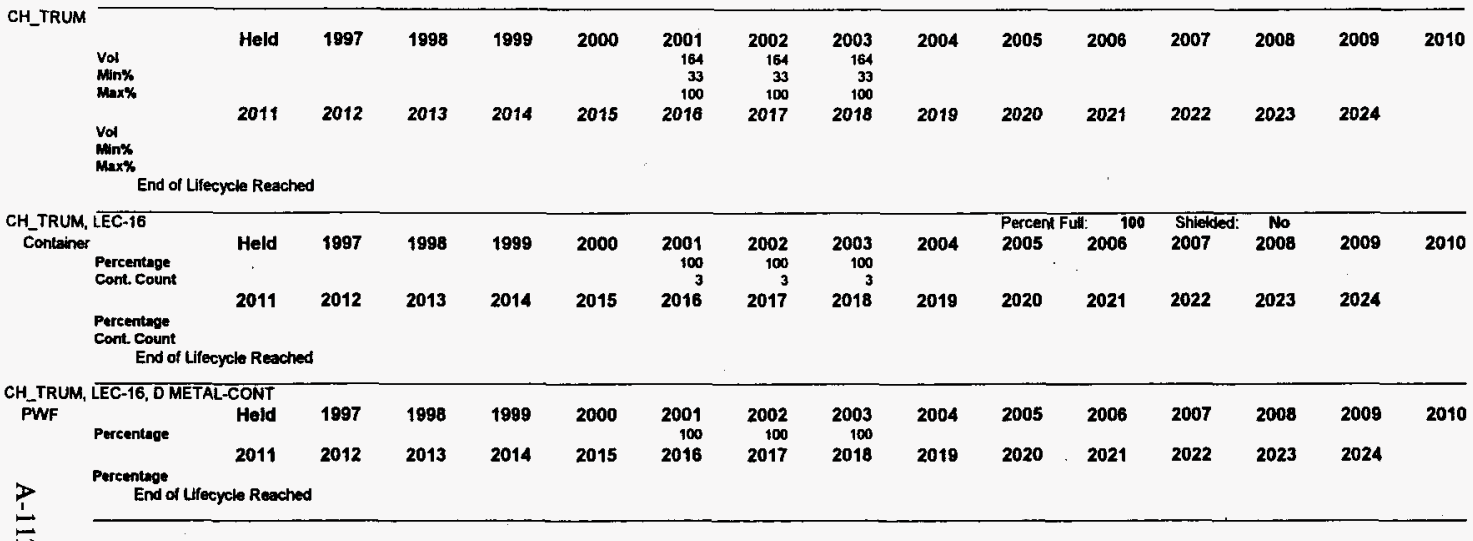




\section{Waste Generator}

Waste Class, Container, Physical Waste Form, (Radionuclide or Hazardous Constituent)

CH_TRUM, LEC-16, D METAL-CONT, WT-WP-WSC2

HC

$\begin{array}{lllllll}\text { Percentage } & \text { Held } & 1997 & 1998 & 1999 & 2000 & 200 \\ & 2011 & 2012 & 2013 & 2014 & 2015 & \\ \text { Percentuge } & & & & & \end{array}$

End of Lifecycle Reached

\begin{tabular}{|c|c|c|c|c|c|c|c|c|c|c|c|c|c|c|c|c|}
\hline$\underset{\text { RAD }}{C H \text { TRUM }}$ & $\begin{array}{l}\text { LEC-16, D MET } \\
\text { Concentration } \\
\text { Concentration } \\
\text { End of Lif }\end{array}$ & $\begin{array}{l}\text { CONT, } \\
\text { Held } \\
2011 \\
\text { cle Rear }\end{array}$ & $\begin{array}{c}\text { TONIUI } \\
1997 \\
2012\end{array}$ & $\begin{array}{r}1998 \\
2013\end{array}$ & $\begin{array}{l}1999 \\
2014\end{array}$ & $\begin{array}{l}2000 \\
2015\end{array}$ & $\begin{array}{r}2001 \\
1 \\
2016\end{array}$ & $\begin{array}{r}2002 \\
1 \\
2017\end{array}$ & $\begin{array}{r}2003 \\
1 \\
2018\end{array}$ & $\begin{array}{l}2004 \\
2019\end{array}$ & $\begin{array}{l}2005 \\
2020\end{array}$ & $\begin{array}{l}2006 \\
2021\end{array}$ & $\begin{array}{l}2007 \\
2022\end{array}$ & $\begin{array}{l}2008 \\
2023\end{array}$ & $\begin{array}{l}2009 \\
2024\end{array}$ & 2010 \\
\hline$\underset{\text { RAD }}{\text { CH_TRUM, }}$ & $\begin{array}{l}\text { LEC-16, OMET } \\
\text { Concentration } \\
\text { Concentratlon } \\
\text { End of Lif }\end{array}$ & $\begin{array}{l}\text { CONT, } \\
\text { Held } \\
2011 \\
\text { cle Reac }\end{array}$ & $\begin{array}{c}\text { SIUM137 } \\
1997 \\
2012\end{array}$ & $\begin{array}{r}1998 \\
2013\end{array}$ & $\begin{array}{r}1999 \\
2014\end{array}$ & $\begin{array}{l}2000 \\
2015\end{array}$ & $\begin{array}{r}2001 \\
10 \\
2016\end{array}$ & $\begin{array}{r}2002 \\
10 \\
2017\end{array}$ & $\begin{array}{r}2003 \\
10 \\
2018\end{array}$ & $\begin{array}{r}2004 \\
2019\end{array}$ & $\begin{array}{l}2005 \\
2020\end{array}$ & $\begin{array}{l}2006 \\
2024\end{array}$ & $\begin{array}{l}2007 \\
2022\end{array}$ & $\begin{array}{l}2008 \\
2023\end{array}$ & $\begin{array}{l}2009 \\
2024\end{array}$ & 2010 \\
\hline$\underset{\text { RAD }}{\mathrm{CH} \text { TRUM, }}$ & $\begin{array}{l}\text { LEC-16, D MET } \\
\text { Concentration } \\
\text { Concentration } \\
\text { End of Lif }\end{array}$ & $\begin{array}{l}\text { CŌNT, } \\
\text { Held } \\
2011 \\
\text { cle Reac }\end{array}$ & $\begin{array}{l}1997 \\
2012\end{array}$ & $\begin{array}{c}1998 \\
2013\end{array}$ & $\begin{array}{c}1999 \\
2014\end{array}$ & $\begin{array}{l}2000 \\
2015\end{array}$ & $\begin{array}{r}2001 \\
10 \\
2016\end{array}$ & $\begin{array}{r}2002 \\
10 \\
2017\end{array}$ & $\begin{array}{r}2003 \\
10 \\
2018\end{array}$ & $\begin{array}{l}2004 \\
2019\end{array}$ & $\begin{array}{l}2005 \\
2020\end{array}$ & $\begin{array}{l}2006 \\
2021\end{array}$ & $\begin{array}{l}2007 \\
2022\end{array}$ & $\begin{array}{l}2008 \\
2023\end{array}$ & $\begin{array}{l}2009 \\
2024\end{array}$ & 2010 \\
\hline & $\begin{array}{l}\text { Vol } \\
\text { Minx } \\
\text { Max\% } \\
\text { Vol } \\
\text { MInx } \\
\text { Max* }\end{array}$ & 2011 & 1997 & 1998 & 1999 & 2000 & $\begin{array}{r}2001 \\
164 \\
33 \\
100 \\
2016\end{array}$ & $\begin{array}{r}2002 \\
164 \\
33 \\
100 \\
2017\end{array}$ & $\begin{array}{r}2003 \\
164 \\
33 \\
100 \\
2018\end{array}$ & 2004 & 2005 & 2006 & 2007 & 2008 & 2009 & 2010 \\
\hline
\end{tabular}

$\frac{1}{1}$ 
Waste Generator

\begin{tabular}{|c|c|c|c|c|c|c|c|c|c|c|c|c|c|c|c|c|}
\hline $\begin{array}{l}\text { RH_TRUM, } \\
\text { Container }\end{array}$ & $\begin{array}{l}\text { LEC-16 } \\
\text { Percentage } \\
\text { Cont. Count } \\
\text { Percentage } \\
\text { Cont. Count } \\
\text { End of L }\end{array}$ & $\begin{array}{l}\text { Held } \\
2011 \\
\text { cle Reac }\end{array}$ & $\begin{array}{r}1997 \\
2012\end{array}$ & $\begin{array}{l}1998 \\
2013\end{array}$ & $\begin{array}{l}1999 \\
2014\end{array}$ & $\begin{array}{l}2000 \\
2015\end{array}$ & $\begin{array}{r}2001 \\
100 \\
3 \\
2016\end{array}$ & $\begin{array}{r}2002 \\
100 \\
3 \\
2017\end{array}$ & $\begin{array}{r}2003 \\
100 \\
3 \\
2018\end{array}$ & $\begin{array}{l}2004 \\
2019\end{array}$ & $\begin{array}{l}\text { Percent Full: } \\
2005 \\
2020\end{array}$ & $\begin{array}{l}2006 \\
2021\end{array}$ & $\begin{array}{l}\text { Shieided: } \\
2007 \\
2022\end{array}$ & $\begin{array}{c}\text { No } \\
2008 \\
\\
2023\end{array}$ & $\begin{array}{l}2009 \\
2024\end{array}$ & 2010 \\
\hline $\begin{array}{c}\text { RH_TRUM, } \\
\text { PWF }\end{array}$ & $\begin{array}{l}\text { LEC-16, O ME } \\
\text { Percentuge } \\
\text { Percentage } \\
\text { End of } \mathrm{L}\end{array}$ & $\begin{array}{l}\text { CONT } \\
\text { Held } \\
2011 \\
\text { cle Reac }\end{array}$ & $\begin{array}{l}1997 \\
2012\end{array}$ & $\begin{array}{l}1998 \\
2013\end{array}$ & $\begin{array}{l}1999 \\
2014\end{array}$ & $\begin{array}{l}2000 \\
2015\end{array}$ & $\begin{array}{r}2001 \\
100 \\
2016\end{array}$ & $\begin{array}{r}2002 \\
100 \\
2017\end{array}$ & $\begin{array}{r}2003 \\
100 \\
2018\end{array}$ & $\begin{array}{l}2004 \\
2019\end{array}$ & $\begin{array}{l}2005 \\
2020\end{array}$ & $\begin{array}{l}2006 \\
2021\end{array}$ & $\begin{array}{l}2007 \\
2022\end{array}$ & $\begin{array}{l}2008 \\
2023\end{array}$ & $\begin{array}{l}2009 \\
2024\end{array}$ & 2010 \\
\hline$\underset{\mathrm{HC}^{\mathrm{R}}}{\mathrm{RH} T R U M}$ & $\begin{array}{l}\text { LEC-16, D ME } \\
\text { Percentuge } \\
\text { Percentuge } \\
\text { End of LI }\end{array}$ & $\begin{array}{l}\text { CONT, } \\
\text { Held } \\
2011 \\
\text { cle Reac }\end{array}$ & $\begin{array}{c}-W P-W S \\
1997 \\
2012\end{array}$ & $\begin{array}{l}1998 \\
2013\end{array}$ & $\begin{array}{l}1999 \\
2014\end{array}$ & $\begin{array}{l}2000 \\
2015\end{array}$ & $\begin{array}{r}2001 \\
100 \\
2016\end{array}$ & $\begin{array}{r}2002 \\
100 \\
2017\end{array}$ & $\begin{array}{r}2003 \\
100 \\
2018\end{array}$ & $\begin{array}{l}2004 \\
2019\end{array}$ & $\begin{array}{l}2005 \\
2020\end{array}$ & $\begin{array}{l}2006 \\
2021\end{array}$ & $\begin{array}{l}2007 \\
2022\end{array}$ & $\begin{array}{l}2008 \\
2023\end{array}$ & $\begin{array}{l}2009 \\
2024\end{array}$ & 2010 \\
\hline$\underset{\text { RAD }}{R H_{\text {_TRUM, }}}$ & $\begin{array}{l}\text { LEC-16, DME } \\
\text { Concentration } \\
\text { Concentration } \\
\text { End of Li }\end{array}$ & $\begin{array}{l}\text { CONT, F } \\
\text { Held } \\
2011 \\
\text { cle React }\end{array}$ & $\begin{array}{l}\text { TONIUM } \\
1997 \\
2012\end{array}$ & $\begin{array}{l}1998 \\
2013\end{array}$ & $\begin{array}{r}1999 \\
2014\end{array}$ & $\begin{array}{l}2000 \\
2015\end{array}$ & $\begin{array}{r}2001 \\
1 \\
2016\end{array}$ & $\begin{array}{r}2002 \\
1 \\
2017\end{array}$ & $\begin{array}{r}2003 \\
1 \\
2018\end{array}$ & $\begin{array}{l}2004 \\
2019\end{array}$ & $\begin{array}{r}2005 \\
2020\end{array}$ & $\begin{array}{l}2006 \\
2021\end{array}$ & $\begin{array}{l}2007 \\
2022\end{array}$ & $\begin{array}{c}2008 \\
2023\end{array}$ & $\begin{array}{l}2009 \\
2024\end{array}$ & 2010 \\
\hline$\underset{\text { RAD }}{\text { RH_TRUM, }}$ & $\begin{array}{l}\text { LEC-16, DME } \\
\text { Concentration } \\
\text { Concentration } \\
\text { End of LI }\end{array}$ & $\begin{array}{l}\text { CONT, C } \\
\text { Held } \\
2011 \\
\text { cle React }\end{array}$ & $\begin{array}{l}\text { TUN137 } \\
1997 \\
2012\end{array}$ & $\begin{array}{l}1998 \\
2013\end{array}$ & $\begin{array}{l}1999 \\
2014\end{array}$ & $\begin{array}{l}2000 \\
2015\end{array}$ & $\begin{array}{r}2001 \\
100 \\
2016\end{array}$ & $\begin{array}{r}2002 \\
100 \\
2017\end{array}$ & $\begin{array}{r}2003 \\
100 \\
2018\end{array}$ & $\begin{array}{l}2004 \\
2019\end{array}$ & $\begin{array}{l}2005 \\
2020\end{array}$ & $\begin{array}{l}2006 \\
2021\end{array}$ & $\begin{array}{l}2007 \\
2022\end{array}$ & $\begin{array}{l}2008 \\
2023\end{array}$ & $\begin{array}{l}2009 \\
2024\end{array}$ & 2010 \\
\hline
\end{tabular}

$\frac{5}{1}$ 


\section{Waste Generator}

Waste Class, Container, Physical Waste Form, (Radionucllde or Hazardous Constituent)

RH_TRUM, LEC-16, D METAL-CONT, STRONTIUMSO

RAD

Concentration

Held 1997

1998

1999

2000

2001

2001
100

100
2016

2002

Concentration

20112012

$20132014 \quad 2015$

2003
100

2004

2005

2006

2007

2008


Waste Generator

Waste Class, Contalner, Physical Waste Form, (Radlonuclide or Hazardous Constituent) WHC SST RE CH_LLW End Of Lifecycle: 2018

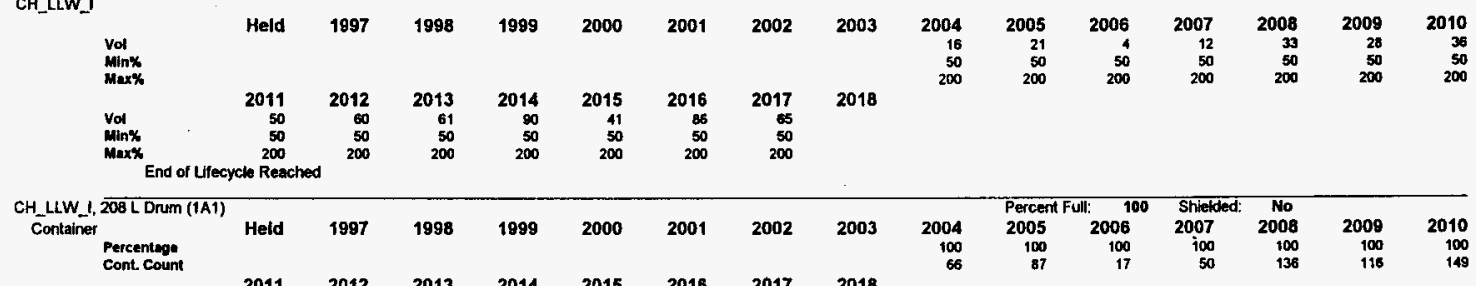

$\begin{array}{rrrrrrrrr} & 2011 & 2012 & 2013 & 2014 & 2015 & 2016 & 2017 & 2018 \\ \text { Percentage } & 100 & 100 & 100 & 100 & 100 & 100 & 100 & \end{array}$

$\begin{array}{lllllll}207 & 248 & 252 & 372 & 169 & 355 & 269\end{array}$

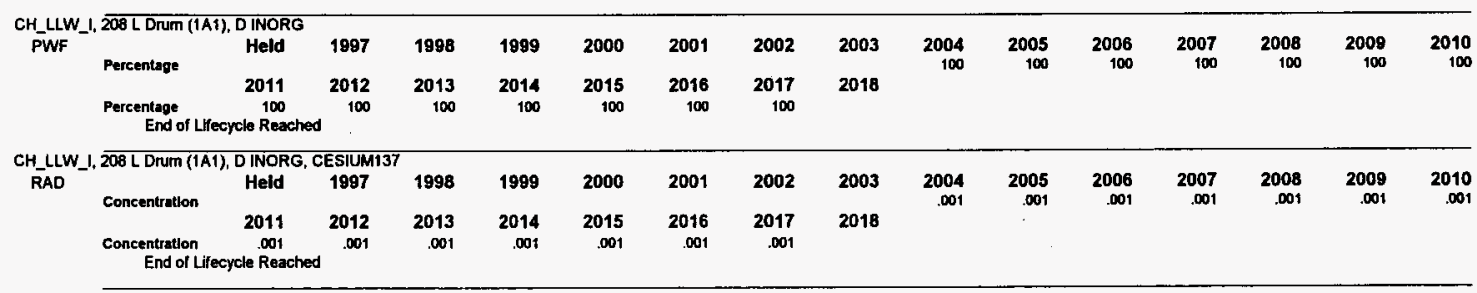

$\frac{1}{2}$ 
Waste Generator

Waste Class, Container, Physical Waste Form, (Radionuclide or Hazardous Constituent)

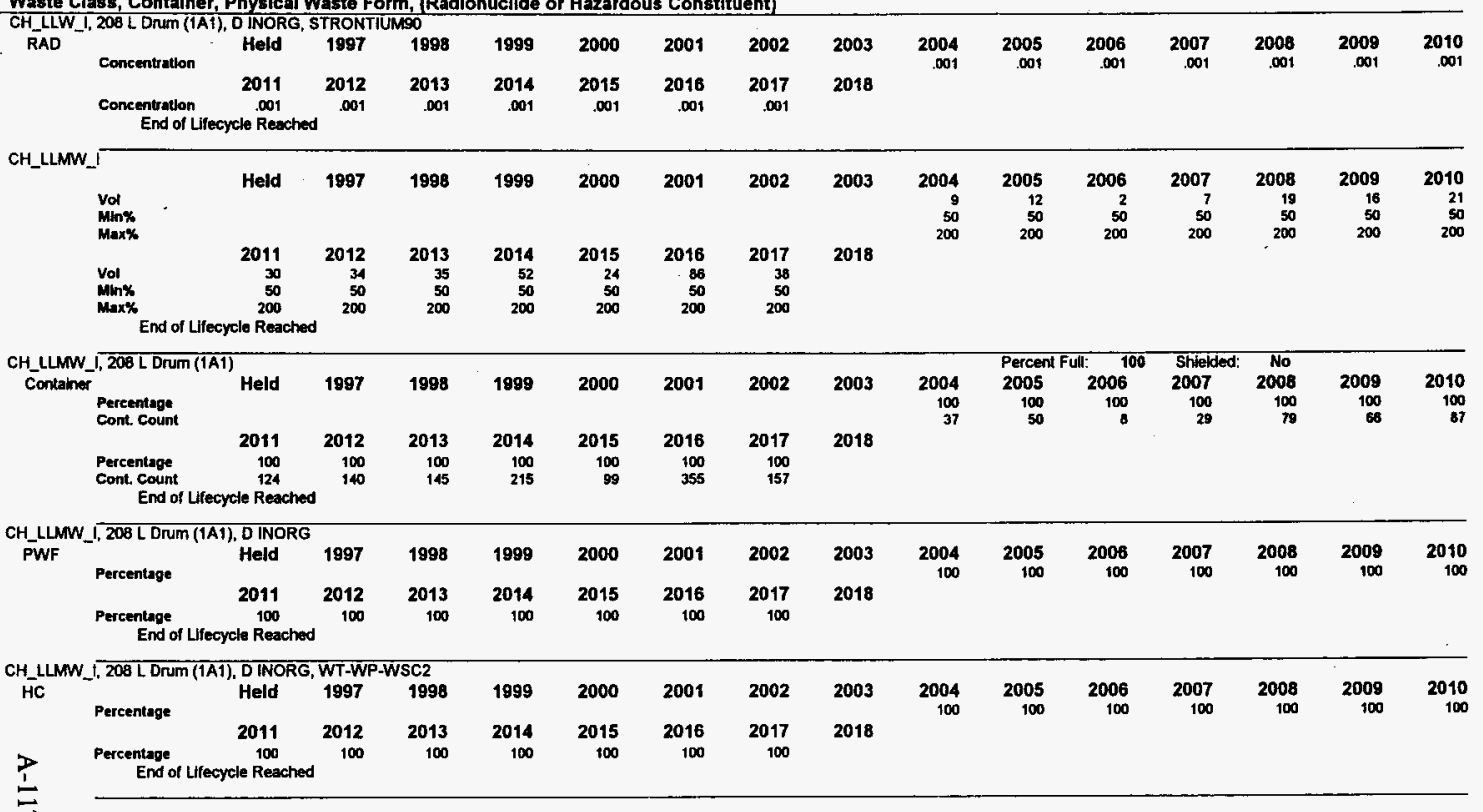


Waste Generator

Waste Class, Container, Physical Waste Form, (Radionuclide or Hazardous Constituent)

Waste Class, Container, Physical Waste Form

$$
\text { RAD }
$$

$\begin{array}{cccc} & & \\ \text { Concentratlon } & \text { Held } & 1997 & 1989 \\ \text { Concentrution } & 2011 & 2012 & 2013 \\ \text { End of Lifecycto Reached } & .001 & .001\end{array}$

$$
1998 \quad 1999
$$

$20002001 \quad 2002$

\begin{tabular}{|c|c|c|c|c|c|c|}
\hline $\begin{array}{r}2004 \\
.001\end{array}$ & $\begin{array}{r}2005 \\
.001\end{array}$ & $\begin{array}{r}2006 \\
.001\end{array}$ & $\begin{array}{r}2007 \\
.001\end{array}$ & $\begin{array}{r}2008 \\
.001\end{array}$ & $\begin{array}{r}2009 \\
.001\end{array}$ & $\begin{array}{r}2010 \\
.001\end{array}$ \\
\hline 2004 & 2005 & $\begin{array}{r}2006 \\
.001\end{array}$ & $\begin{array}{r}2007 \\
.001\end{array}$ & $\begin{array}{r}2006 \\
.001\end{array}$ & $\begin{array}{r}2009 \\
.001\end{array}$ & $\begin{array}{r}2010 \\
.001\end{array}$ \\
\hline
\end{tabular}

20022003

\begin{tabular}{|c|c|c|c|c|c|c|c|c|c|c|c|c|c|c|c|c|}
\hline RAD & $\begin{array}{l}\text { Concentration } \\
\text { Concentration } \\
\text { End of Life }\end{array}$ & $\begin{array}{l}\text { Held } \\
2011 \\
.001 \\
\text { cle Reached }\end{array}$ & $\begin{array}{r}1997 \\
2012 \\
.001\end{array}$ & $\begin{array}{r}1998 \\
2013 \\
.001\end{array}$ & $\begin{array}{r}1999 \\
2014 \\
.001\end{array}$ & $\begin{array}{r}2000 \\
2015 \\
.001\end{array}$ & $\begin{array}{r}2001 \\
2016 \\
.001\end{array}$ & $\begin{array}{r}2002 \\
2017 \\
.001\end{array}$ & $\begin{array}{l}2003 \\
2018\end{array}$ & $\begin{array}{r}2004 \\
.001\end{array}$ & $\begin{array}{r}2005 \\
.001\end{array}$ & $\begin{array}{r}2006 \\
.001\end{array}$ & $\begin{array}{r}2007 \\
.001\end{array}$ & $\begin{array}{r}2008 \\
.001\end{array}$ & $\begin{array}{r}2009 \\
.001\end{array}$ & $\begin{array}{r}2010 \\
.001\end{array}$ \\
\hline & $\begin{array}{l}\text { Vol } \\
\min x \\
\operatorname{Max\% }\end{array}$ & Held & 1997 & 1998 & 1999 & 2000 & 2001 & 2002 & 2003 & $\begin{array}{r}2004 \\
14 \\
50 \\
200\end{array}$ & $\begin{array}{r}2005 \\
17 \\
50 \\
200\end{array}$ & $\begin{array}{r}2006 \\
4 \\
50 \\
200\end{array}$ & $\begin{array}{r}2007 \\
10 \\
50 \\
200\end{array}$ & $\begin{array}{r}2008 \\
27 \\
50 \\
200\end{array}$ & $\begin{array}{r}2009 \\
24 \\
50 \\
200\end{array}$ & $\begin{array}{r}2010 \\
31 \\
50 \\
200\end{array}$ \\
\hline
\end{tabular}

CH_LLMN_1, 208 L Drum (IA1), D INORG, STRONTIUMGO

\begin{tabular}{|c|c|c|c|c|c|c|c|c|}
\hline $\begin{array}{l}\text { Vod } \\
\operatorname{Min} x \\
\operatorname{Max} \%\end{array}$ & $\begin{array}{r}2011 \\
40 \\
50 \\
200\end{array}$ & $\begin{array}{r}2012 \\
52 \\
50 \\
200\end{array}$ & $\begin{array}{r}2013 \\
51 \\
50 \\
200\end{array}$ & $\begin{array}{r}2014 \\
76 \\
50 \\
200\end{array}$ & $\begin{array}{r}2015 \\
34 \\
50 \\
200\end{array}$ & $\begin{array}{r}2016 \\
72 \\
50 \\
200\end{array}$ & $\begin{array}{r}2017 \\
55 \\
50 \\
200\end{array}$ & 2018 \\
\hline
\end{tabular}

\begin{tabular}{|c|c|c|c|c|c|c|c|}
\hline & 2011 & 2012 & 2013 & 2014 & 2015 & 2016 & 2017 \\
\hline $\begin{array}{l}\text { Percentuge } \\
\text { Cont Count }\end{array}$ & $\begin{array}{l}100 \\
165\end{array}$ & $\begin{array}{l}100 \\
215\end{array}$ & $\begin{array}{l}100 \\
211\end{array}$ & $\begin{array}{l}100 \\
314\end{array}$ & $\begin{array}{l}100 \\
140\end{array}$ & $\begin{array}{l}100 \\
290\end{array}$ & $\begin{array}{l}100 \\
227\end{array}$ \\
\hline
\end{tabular}

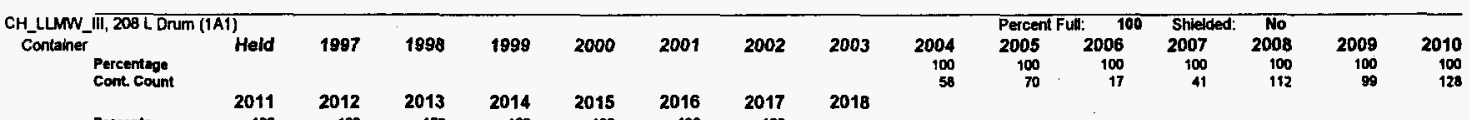

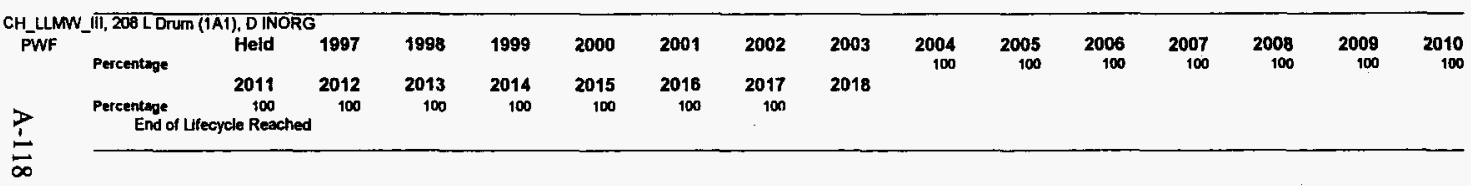


Waste Generator

Waste Class, Container, Physical Waste Form, (Radionucllde or Hazardous Constituent)

CH_LLMW_III, $20 \bar{B}$ L ÖUTU (1A1), D INORG, WT-WP-WSC2

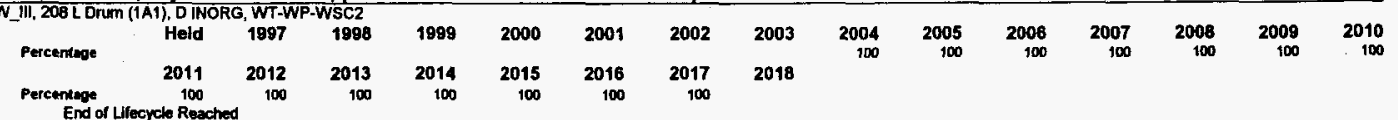

$$
\text { End of Lifecycle Reached }
$$

\begin{tabular}{|c|c|c|c|c|c|c|c|c|c|c|c|c|c|c|c|c|}
\hline \multicolumn{17}{|c|}{ CH_LLMW_III, 208 L Drum (1Āi), D INORG, CESIUMi37 } \\
\hline \multirow[t]{3}{*}{$\overline{R A D}$} & Concentrution & & 1997 & 1998 & & 2000 & 2001 & & & $\begin{array}{r}2004 \\
10\end{array}$ & $\begin{array}{r}2005 \\
10\end{array}$ & $\begin{array}{r}2006 \\
10\end{array}$ & $\begin{array}{r}2007 \\
10\end{array}$ & $\begin{array}{r}2008 \\
10\end{array}$ & $\begin{array}{r}2009 \\
10\end{array}$ & $\begin{array}{r}2010 \\
10\end{array}$ \\
\hline & & 2011 & 2012 & 2013 & 2014 & 2015 & 2016 & 2017 & 2018 & & & & & & & \\
\hline & $\begin{array}{l}\text { Concentration } \\
\text { End of Life }\end{array}$ & rele Rea & 10 & 10 & 10 & 10 & 10 & 10 & & & & & & & & \\
\hline \multirow{4}{*}{$\underset{\text { RAD }}{\text { CH_LLMN }}$} & III, 208 L Drum ( & 1), DIN & 3, STRO & UM9O & & & & & & & & & & & & \\
\hline & & Held & 1997 & 1998 & 1999 & 2000 & 2001 & 2002 & 2003 & $\begin{array}{r}2004 \\
10\end{array}$ & $\begin{array}{r}2005 \\
10\end{array}$ & $\begin{array}{r}2006 \\
10\end{array}$ & $\begin{array}{r}2007 \\
10\end{array}$ & $\begin{array}{r}2008 \\
10\end{array}$ & $\begin{array}{r}2009 \\
10\end{array}$ & $\begin{array}{r}2010 \\
10\end{array}$ \\
\hline & & 2011 & 2012 & 2013 & 2014 & 2015 & 2016 & 2017 & 2018 & & & & & & & \\
\hline & Concentration & 10 & 10 & 10 & to & 10 & 10 & 10 & & & & & & & & \\
\hline
\end{tabular}

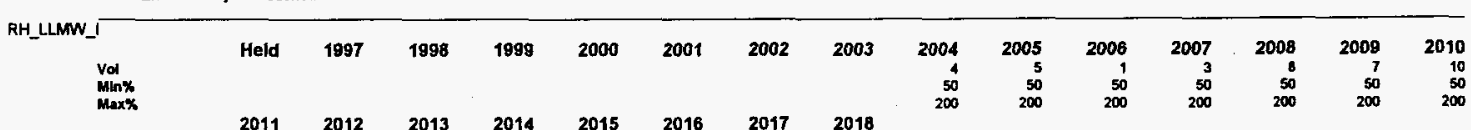

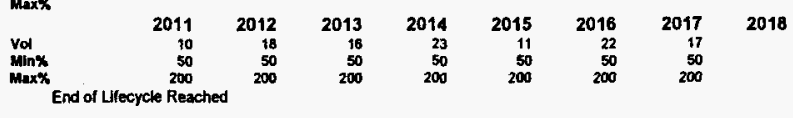

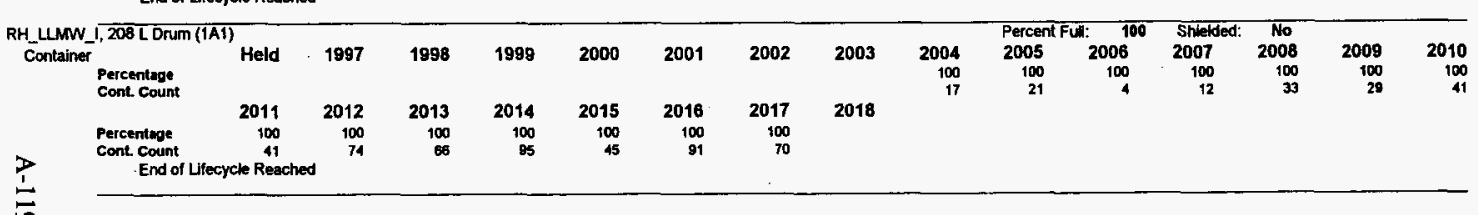


Complete Data Summary

Waste Generator

Waste Class, Container, Physical Waste Form, (Radionuclide or Hazardous Constituent)

RH_LLMW_L, 208 . L Drum (1A1), D INORG

$\begin{array}{lrrrrrrr}\text { Percentuge } & \text { Held } & 1997 & 1998 & 1989 & 2000 & 2001 & 2002 \\ & 2011 & 2012 & 2013 & 2014 & 2015 & 2016 & 2017 \\ \text { Pereentage } & \begin{array}{ll}100 \\ \text { End of Lifecyclo Reached }\end{array} & 100 & 100 & 100 & 100 & 100 & 100\end{array}$

1004

2003

2004

2005

2006

RH_LLMW_I, 208 L Drum (1A1), D INORG, WT-WP-WSC2

\begin{tabular}{|c|c|c|c|c|c|c|c|c|c|c|c|c|c|c|c|c|}
\hline \multirow{4}{*}{$\mathrm{HC}_{\mathrm{HC}}$} & 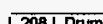 & ח & 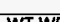 & 1092 & & & & & & & & & & & & \\
\hline & \multirow[t]{2}{*}{ Dercenteng } & & , WT-W & ISC2 & 1999 & & 2001 & 2002 & & 2004 & 2005 & 2006 & 2007 & 2008 & 2009 & 2010 \\
\hline & & 2011 & (2012, & 2013 & 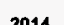 & 2015 & 2018 & 2017 & 2018 & & & & & & & \\
\hline & Percentage & $\begin{array}{r}2011 \\
100\end{array}$ & $\begin{array}{r}2012 \\
100\end{array}$ & 2013 & $\begin{array}{r}2014 \\
100\end{array}$ & 2015 & $\begin{array}{r}2016 \\
100\end{array}$ & $\begin{array}{r}2017 \\
100\end{array}$ & & & & & & & & \\
\hline
\end{tabular}
End of Lifecycle Reached

RH_LLMW_I, 208 L DWU (1A1), D INORG, CESIUM137

\begin{tabular}{|c|c|c|c|c|c|c|c|c|c|c|c|c|c|c|c|c|}
\hline RAD & $\begin{array}{l}\text { 1, 208 L Drum (1 } \\
\text { Concentration } \\
\text { concentration } \\
\text { End of Lif }\end{array}$ & $\begin{array}{l}\text { D D INO } \\
\text { Held } \\
2011 \\
100 \\
\text { rele Reac }\end{array}$ & $\begin{array}{r}1997 \\
2012 \\
100\end{array}$ & $\begin{array}{r}1998 \\
2013 \\
100\end{array}$ & $\begin{array}{r}1999 \\
2014 \\
100\end{array}$ & $\begin{array}{r}2000 \\
2015 \\
100\end{array}$ & $\begin{array}{r}2001 \\
2016 \\
100\end{array}$ & $\begin{array}{r}2002 \\
2017 \\
100\end{array}$ & $\begin{array}{l}2003 \\
2018\end{array}$ & $\begin{array}{r}2004 \\
100\end{array}$ & $\begin{array}{r}2005 \\
100\end{array}$ & $\begin{array}{r}2006 \\
100\end{array}$ & $\begin{array}{r}2007 \\
100\end{array}$ & $\begin{array}{r}2008 \\
100\end{array}$ & $\begin{array}{r}2009 \\
100\end{array}$ & $\begin{array}{r}2010 \\
100\end{array}$ \\
\hline RAD & $\begin{array}{l}\text { 1., } 208 \text { L Drum (1 } \\
\text { Concentration } \\
\text { Concentration } \\
\text { End of Lif }\end{array}$ & $\begin{array}{r}\text { \% D INO } \\
\text { Held } \\
2011 \\
\text { to0 } \\
\text { cle Reac }\end{array}$ & $\begin{array}{r}\text { STROA } \\
1997 \\
2012 \\
100\end{array}$ & $\begin{array}{l}\text { JM90 } \\
1998 \\
2013 \\
100\end{array}$ & $\begin{array}{r}1999 \\
2014 \\
100\end{array}$ & $\begin{array}{r}2000 \\
2015 \\
100\end{array}$ & $\begin{array}{r}2001 \\
2016 \\
100\end{array}$ & $\begin{array}{r}2002 \\
2017 \\
100\end{array}$ & $\begin{array}{l}2003 \\
2018\end{array}$ & $\begin{array}{r}2004 \\
100\end{array}$ & $\begin{array}{r}2005 \\
100\end{array}$ & $\begin{array}{r}2006 \\
100\end{array}$ & $\begin{array}{r}2007 \\
100\end{array}$ & $\begin{array}{r}2008 \\
100\end{array}$ & $\begin{array}{r}2009 \\
100\end{array}$ & $\begin{array}{r}2010 \\
100\end{array}$ \\
\hline & $\begin{array}{l}\text { Vol } \\
\text { Minx } \\
M \mathbf{2 x}\end{array}$ & Held & 1987 & 1998 & 1999 & 2000 & 2001 & 2002 & 2003 & $\begin{array}{r}2004 \\
6 \\
50 \\
200\end{array}$ & $\begin{array}{r}2005 \\
8 \\
50 \\
200\end{array}$ & $\begin{array}{r}2006 \\
2 \\
50 \\
200\end{array}$ & $\begin{array}{r}2007 \\
5 \\
50 \\
200\end{array}$ & $\begin{array}{r}2008 \\
13 \\
50 \\
200\end{array}$ & $\begin{array}{r}2009 \\
11 \\
50 \\
200\end{array}$ & $\begin{array}{r}2010 \\
14 \\
50 \\
200\end{array}$ \\
\hline
\end{tabular}

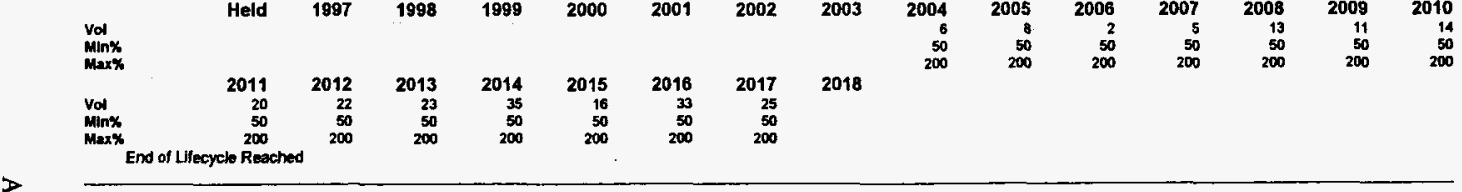

苞 
Waste Generator

Waste Class, Container, Phyeical Waste Form, (Radionuclide or Hazardous Constituent)

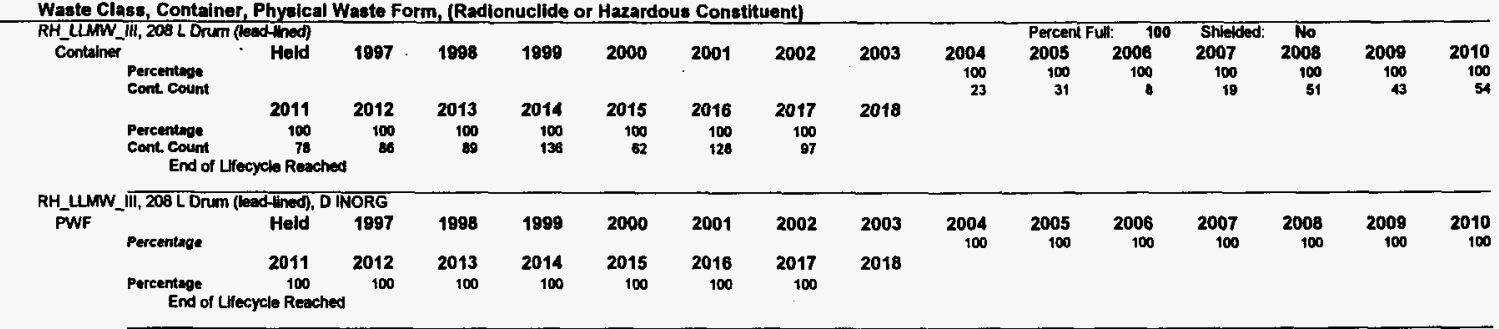




\section{WHC-SD-WM-TI-777}

\section{Revision 0}

This page intentionally left blank. 
WHC-SD-WM-TI-777

Revision 0

APPENDIX B

SINGLE-SHELL TANK EQUIPMENT LIST

B-1 
WHC-SD-WM-TI-777

Revision 0

This page intentionally left blank.

B-2 
WHC-SD-WM-TI-777

Revision 0

Single-Shell Tank Equipment List. (3 sheets)

\begin{tabular}{|c|c|c|c|c|c|c|c|c|c|c|c|c|c|c|c|c|c|c|}
\hline$\supset 0$ & & & & & & - & 유 & $m$ & $\forall$ & & $a$ & & & $N$ & $\sim$ & & $\infty$ & $\infty$ \\
\hline$\tau \theta$ & & & & & & & & & & & $\nabla$ & & & & - & & $\sim$ & $\rightarrow$ \\
\hline$\not \stackrel{\infty}{=}$ & $\underline{m}$ & & & & & & & & & & $n$ & & & + & 6 & $\Rightarrow$ & - & 士 \\
\hline$+\theta$ & & & & & & & & & $m$ & & \pm & & & & - & & $N$ & $m$ \\
\hline$\sqrt[x]{n}$ & 음 & & - & $N$ & - & & . & $m$ & & & 0 & & & & & & 으 & $n$ \\
\hline n $\overparen{\exists}$ & & & & $N$ & & & & & & & $\simeq$ & & & & & & $\sim$ & 으 \\
\hline u@ & & & & & & & 으 & $N$ & - & & $n$ & - & & & & 0 & 0 & $m$ \\
\hline$\vec{\Delta} \Xi$ & $\underline{-}$ & - & & & & & - & & & - & - & & & - & & - & $a$ & $\infty$ \\
\hline$\underset{\oplus \Xi}{\Xi}$ & & & & & & & $\infty$ & & & & $\infty$ & & & in & $N$ & -1 & $\forall$ & - \\
\hline$\infty \stackrel{6}{=}$ & & & & & & -1 & $\theta$ & & & & - & & & $\sim$ & - & 0 & $m$ & $N$ \\
\hline$\stackrel{x}{\dot{E}}$ & $\infty$ & & & & & & & & $\stackrel{\infty}{\sim}$ & & $\nabla$ & & & & & $\sim$ & & - \\
\hline$<9$ & $n$ & & & & $\nabla$ & - & & & $n$ & & $\nabla$ & & $N$ & & $N$ & & -1 & $N$ \\
\hline 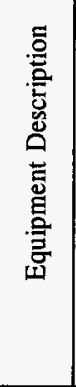 & 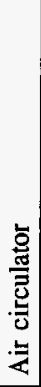 & 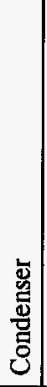 & 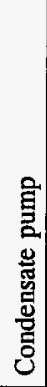 & 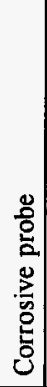 & 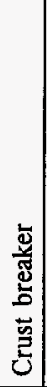 & 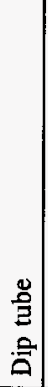 & 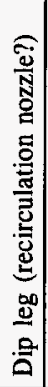 & 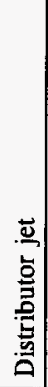 & 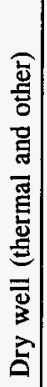 & 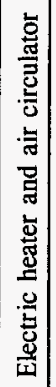 & $\stackrel{\underline{I}}{\underline{I}}$ & 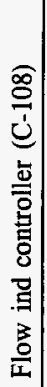 & 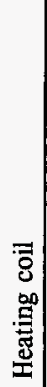 & 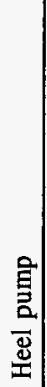 & 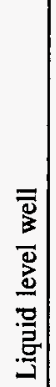 & 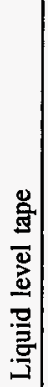 & 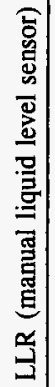 & 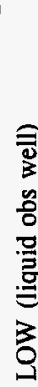 \\
\hline
\end{tabular}




\section{WHC-SD-WM-TI-777 \\ Revision 0}

Single-Shell Tank Equipment List. (3 sheets)

\begin{tabular}{|c|c|c|c|c|c|c|c|c|c|c|c|c|c|c|c|c|c|}
\hline$D Q$ & & $\because$ & & & & & - & & & & $\infty$ & & & $m$ & & $\infty$ & \\
\hline$\gg \theta$ & & $n$ & & & $N$ & & $\sim$ & & & & & & -1 & - & & & \\
\hline$\underset{⿴ 囗 大}{=}$ & & $m$ & & & & $\forall$ & $\simeq$ & - & & -1 & & & & -1 & & + & - \\
\hline$-\theta$ & & $=$ & & & & & - & 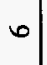 & & -1 & & & & & & -1 & \\
\hline 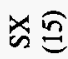 & & $\Xi$ & & & $a$ & N & $n$ & & - & & & & & & $\infty$ & & \\
\hline$\sim \widehat{\Theta}$ & & $m$ & & $N$ & -1 & & $\approx$ & & & & & & & $\stackrel{\infty}{\sim}$ & & & \\
\hline ن & & 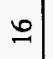 & & & $m$ & & $\nabla$ & $m$ & & & $\theta$ & $\theta$ & & & & - & \\
\hline 它 & $N$ & 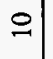 & - & & $m$ & & $r$ & & & & & & + & & & & - \\
\hline$\underset{\infty}{\Xi}$ & & $\simeq$ & $\nabla$ & & $\nabla$ & & 0 & & & & - & & -1 & & & $\infty$ & \\
\hline$\triangle \stackrel{6}{=}$ & & 이 & - & & $m$ & & $a$ & & & & & & - & & & $\nabla$ & \\
\hline$\hat{x} \hat{\theta}$ & & $\theta$ & 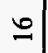 & & & & $m$ & & & & & & $a$ & & & $\sim$ & \\
\hline$<9$ & & $n$ & & & $\infty$ & & $\sim$ & & & & & & $n$ & $N$ & - & -1 & \\
\hline 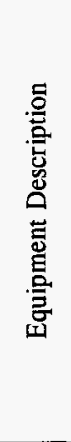 & 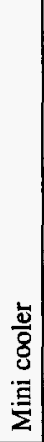 & 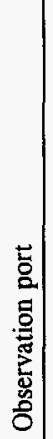 & 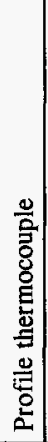 & 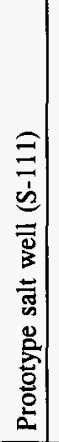 & 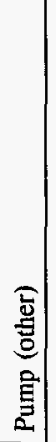 & 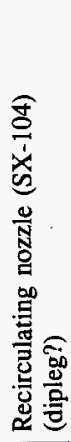 & 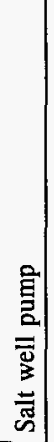 & $\begin{array}{l}\overline{\bar{d}} \\
\overline{3} \\
\bar{\omega} \\
\text { ஸे }\end{array}$ & 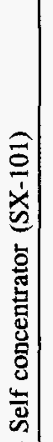 & 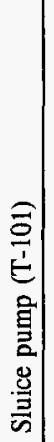 & 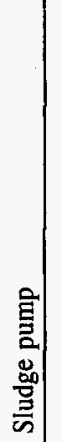 & 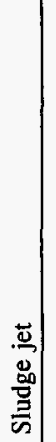 & 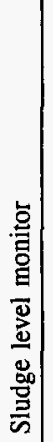 & 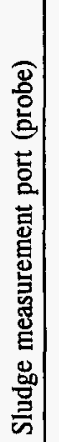 & 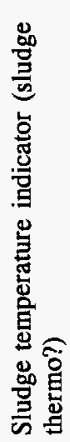 & 密 & $\begin{array}{l}\text { 㽞 } \\
\text { ह } \\
\text { 莺 }\end{array}$ \\
\hline
\end{tabular}


WHC-SD-WM-TI-777

Revision 0

Single-Shell Tank Equipment List. (3 sheets)

\begin{tabular}{|c|c|c|c|c|c|c|c|c|c|c|c|c|c|}
\hline$\supset \stackrel{G}{=}$ & & & & & & & & & & \pm & & & \\
\hline$\geq 6$ & & & & & & & & & & $n$ & & & \\
\hline$\underset{x}{\infty} \stackrel{\infty}{=}$ & & & & - & & & & & & $\mathscr{0}$ & & & \\
\hline$\leftarrow \stackrel{6}{=}$ & & & & & & & & & & $\Rightarrow$ & N & $\theta$ & \\
\hline$\underset{n}{n}=$ & - & & & & & & & & $N$ & S & & & \\
\hline$\sim \overparen{\overparen{\Xi}}$ & 이 & & & & & & & -1 & & $\cong$ & & & \\
\hline $0 \stackrel{6}{=}$ & & - & & & & & $\infty$ & & & $\infty$ & & & \\
\hline 正 & & & & - & & & & & & in & & & -1 \\
\hline$\underset{\infty}{\infty}$ & & & & & & & & & & 옹 & & & \\
\hline$m \stackrel{6}{=}$ & & & & & & & & & & 2 & & & - \\
\hline$\underset{z}{*}$ & & & $m$ & & $N$ & & I & & & $\nabla$ & & & \\
\hline 46 & - & & & & & & & & & \pm & & & \\
\hline 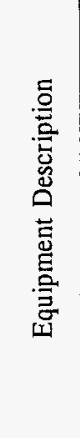 & 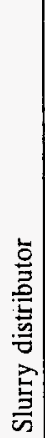 & 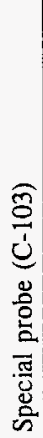 & 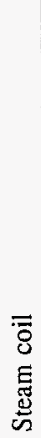 & 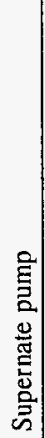 & 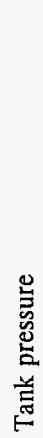 & 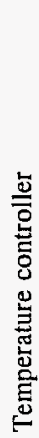 & 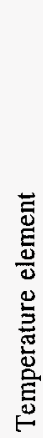 & 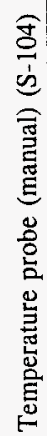 & 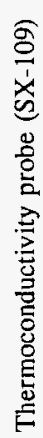 & 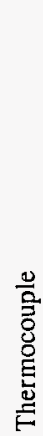 & 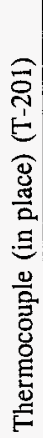 & 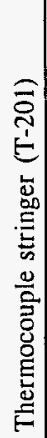 & 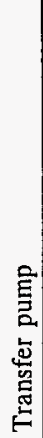 \\
\hline
\end{tabular}




\section{WHC-SD-WM-TI-777}

\section{Revision 0}

This page intentionally left blank.

B-6 


\begin{tabular}{|c|c|c|c|c|c|}
\hline \multirow{2}{*}{$\begin{array}{l}\text { To } \\
\text { Distribution }\end{array}$} & \multirow{2}{*}{\multicolumn{3}{|c|}{$\begin{array}{l}\text { From } \\
\text { G. W. Reddick }\end{array}$}} & \multicolumn{2}{|c|}{ Page 1 of 1} \\
\hline & & & & \multicolumn{2}{|c|}{ Date $8 / 27 / 96$} \\
\hline \multirow{2}{*}{\multicolumn{4}{|c|}{$\begin{array}{l}\text { Project Title/Work Order } \\
\text { Bases for Sol id Waste Volume Estimates for Tank Waste } \\
\text { Remediation System, WHC-SD-WM-TI-777, Rev. 0 }\end{array}$}} & \multicolumn{2}{|c|}{ EDT No. $\quad 617628$} \\
\hline & & & & \multicolumn{2}{|c|}{ ECN No. } \\
\hline Name & MSIN & $\begin{array}{l}\text { Text } \\
\text { With All } \\
\text { Attach. }\end{array}$ & Text Only & $\begin{array}{c}\text { Attach./ } \\
\text { Appendix } \\
\text { Only }\end{array}$ & $\begin{array}{l}\text { EDT/ECN } \\
\text { Only }\end{array}$ \\
\hline $\begin{array}{l}\text { Central Files (2) } \\
\text { DOE Reading Room }\end{array}$ & $\begin{array}{l}A 3-88 \\
A 1-65\end{array}$ & $\begin{array}{l}x \\
x\end{array}$ & & & \\
\hline $\begin{array}{l}\text { S. K. Baker } \\
\text { P. A. Gagnon } \\
\text { J. S. Garfield } \\
\text { J. O. Honeyman } \\
\text { E. J. Kosiancic } \\
\text { R. P. Marshall } \\
\text { G. W. Reddick (3) } \\
\text { O. J. Valero }\end{array}$ & $\begin{array}{l}H 5-49 \\
S 6-31 \\
H 5-49 \\
G 3-21 \\
H 5-61 \\
H 5-61 \\
H 5-49 \\
T 3-01\end{array}$ & $\begin{array}{l}x \\
x \\
x \\
x \\
x \\
x \\
x \\
x\end{array}$ & & & \\
\hline
\end{tabular}

